\title{
Estudo das contribuições térmica e eletrônica na variação do índice de refração de materiais dopados com íons emissores
}

\author{
Djalmir Nestor Messias
}

Tese apresentada ao Instituto de Física de São Carlos, da Universidade de São Paulo, para a obtenção do título de Doutor em Ciências: Física Básica

Orientador: Prof. Dr. Tomaz Catunda 


Messias, Djalmir Nestor
"Estudo das contribuições térmica e eletrônica na variação do índice de
refração de materiais dopados com íons emissores"
Djalmir Nestor Messias - São Carlos, 2006
Tese (Doutorado) - Área de Física Básica do Instituto de Física de São Carlos
da Universidade de São Paulo
2006 - Páginas: 147
Orientador: Prof. Dr. Tomaz Catunda
1. Lente Térmica; 2. Lente de População; 3. Propriedades óticas não lineares
I. Título


"Tantas vezes pensamos ter chegado, tantas vezes é preciso ir além."

Fernando Pessoa 
À Lanna e Andréa, farol e porto, que enchem de luz e alegria a minha vida. 


\section{Agradecimentos}

Como todo bom nordestino gostaria de iniciar agradecendo a Deus pelas portas que encontrei abertas e por aquelas que tive de abrir.

Aos meus pais, meus maiores mestres, por seus incentivos, amor e orientação em todos os momentos importantes na minha vida.

Ao amigo João Francisco, cuja distância nunca se tornou ausência.

Aos novos e antigos amigos colecionados nesta jornada. À Gláucia, Marcos, Renato, Acácio e Rosimeire, Carlos, Sandro, Heitor, Rui, Ester, Andréa, Paulo César, Maria José, Newton, Sr João Francisco.

Ao Prof. Tomaz Catunda por sua orientação durante o desenvolvimento deste trabalho.

A todos os colegas e funcionários deste instituto que direta ou indiretamente contribuíram para minha formação.

E finalmente à FAPESP pelo apoio financeiro, sem o qual este trabalho não poderia ter sido feito. 


\section{Índice}

\section{Capítulo 1 - Introdução geral}

1.1 Introdução geral

1.2 Referências 5

Capítulo 2 - Medidas de propriedades fototérmicas de vidros dopados com 7

$\mathbf{Y b}^{3+}$

2.1 Efeito e a técnica de Lente Térmica $\quad 7$

$\begin{array}{ll}\text { 2.2.1Técnica de LT } & 10\end{array}$

$\begin{array}{ll}\text { 2.1.2 Arranjo Experimental } & 14\end{array}$

2.1.3 Aplicação da técnica de lente térmica a um vidro germanato dopado com $\mathrm{Yb}^{3} \quad 16$

$\begin{array}{ll}\text { 2.2 Espectroscopia de LT no vidro QX/Yb } & 19\end{array}$

$\begin{array}{ll}2.3 \text { Espectroscopia de LT no vidro aluminato } & 24\end{array}$

$\begin{array}{ll}2.4 \text { Conclusões do capítulo } & 28\end{array}$

2.5 Referências 29

\section{Capítulo 3 - Efeitos populacionais sobre o índice de refração não linear}

3.1 Dependência do índice de refração não linear com a população do estado excitado 33

$\begin{array}{ll}3.1 .1 \text { A técnica de z-scan } & 38\end{array}$

3.1.2 A técnica de z-scan resolvida no tempo 43

3.1.3 Arranjo experimental da técnica de z-scan resolvida no tempo 45

3.1.4 Medidas de z-scan em vidro fosfato dopado com $\mathrm{Yb}^{3+} \quad 46$

3.2 Distâncias focais induzidas devido aos efeitos térmico e eletrônico 49 


\section{Capítulo 4 - Efeitos de saturação sobre as medidas de z-scan}

4.1 Efeitos de Saturação sobre as medidas de z-scan $\quad 54$

$\begin{array}{ll}\text { 4.2 Medidas saturadas no cristal de rubi } & 57\end{array}$

4.2.1 Análise do efeito da saturação sobre as medidas transientes 57

4.2.2 O efeito de alargamento na curva de z-scan induzido pela saturação 63

4.2.3 Determinação dos parâmetros não lineares no rubi $\quad 67$

$\begin{array}{ll}4.3 \text { Conclusões do capítulo } & 73\end{array}$

$\begin{array}{ll}4.4 \text { Referências } & 74\end{array}$

\section{Capítulo 5 - Determinação do parâmetro de upconversion Auger através da} técnica de z-scan

5.1 Dinâmica do processo de upconversion Auger

5.2 Excitação não ressonante

5.3 Excitação ressonante 
Capítulo 6 - Espectroscopia eletrônica em amostra vítrea dopada com Yb $\mathbf{b}^{3+} \quad 110$

$\begin{array}{ll}\text { 6.1 Espectroscopia eletrônica no vidro QX/Yb } & 111\end{array}$

$\begin{array}{ll}\text { 6.1.1 Aparato experimental da espectroscopia não linear } & 116\end{array}$

$\begin{array}{ll}\text { 6.1.2 Determinação de } I_{S} \text { e } \tau_{0} & 118\end{array}$

6.1.3 Determinação do espectro de $n_{2} \quad 119$

$\begin{array}{ll}\text { 6.2 Determinação da magnitude das contribuições térmica e eletrônica } & 125\end{array}$

$\begin{array}{ll}\text { 6.3 Conclusões do capítulo } & 127\end{array}$

$\begin{array}{ll}6.4 \text { Referências } & 128\end{array}$

$\begin{array}{ll}\text { Capítulo } 7 \text { - Conclusões gerais e perspectivas } & 131\end{array}$

Apêndice A - Características espectroscópicas de sólidos dopados com $\mathbf{C r}^{\mathbf{3 +}}, \quad 133$

$\mathrm{Nd}^{3+} \mathbf{e} \mathbf{Y b}^{3+}$

$\begin{array}{ll}\text { A.1 Materiais dopados com } \mathrm{Cr}^{3+} & 133\end{array}$

$\begin{array}{ll}\text { A.2 Materiais dopados com } \mathrm{Nd}^{3+} & 136\end{array}$

$\begin{array}{ll}\text { A.3 Materiais dopados com } \mathrm{Yb}^{3+} & 139\end{array}$

Apêndice B - Integral de difração de Kirchhoff 141

Apêndice C - Medidas das cinturas dos feixes de prova e excitação 146 


\section{Lista de Símbolos}

D difusividade térmica

K condutividade térmica

$\rho \quad$ densidade volumétrica

C calor específico

$\eta \quad$ eficiência quântica de fluorescência

$\boldsymbol{t}_{\boldsymbol{c}} \quad$ constante de tempo característica de formação da lente térmica

$\boldsymbol{\theta}$ amplitude do sinal da lente térmica

$\boldsymbol{d s} / \mathbf{d T}$ taxa de variação do caminho ótico com a temperatura

$\boldsymbol{d n} / \boldsymbol{d T}$ taxa de variação do índice de refração com a temperatura

n índice de refração

$\boldsymbol{n}_{\boldsymbol{o}} \quad$ índice de refração na ausência da radiação

$\boldsymbol{n}_{2}$ índice de refração não-linear

$f_{L} \quad$ fator de Lorentz

$\boldsymbol{w}_{\boldsymbol{o}}$ raio mínimo do feixe de excitação (na posição da amostra)

$w_{p}$ raio do feixe de prova na posição da amostra

$\mathbf{z}_{c} \quad$ parâmetro confocal

$\mathbf{z}_{\boldsymbol{c}}$ parâmetro confocal do feixe de prova

$\mathbf{z}_{1}$ distância entre as cinturas dos feixes de prova e de excitação

$\mathbf{z}_{2}$ distância entre a amostra e o detetor no campo distante

$\lambda_{p} \quad$ comprimento de onda do feixe de prova

$\lambda$ comprimento de onda do feixe de excitação

$<\lambda_{e m}^{-1}>$ valor médio do inverso do comprimento de onda de emissão

$\boldsymbol{P}$ potência de excitação

$\boldsymbol{a}_{a b s}$ coeficiente de absorção

L espessura da amostra

$\boldsymbol{L}_{\boldsymbol{e f}} \quad$ espessura efetiva da amostra

$\phi \quad$ fração de energia absorvida convertida em calor

$\boldsymbol{R}$ refletância

$\gamma \quad$ parâmetro de upconversion 
$\alpha_{g} \quad$ polarizabilidade do estado fundamental

$\boldsymbol{\alpha}_{\boldsymbol{e x}} \quad$ polarizabilidade do estado excitado

$\Delta \alpha$ diferença de polarizabilidade entre os estados excitado e fundamental

$v \quad$ razão de Poisson

$\boldsymbol{E} \quad$ módulo de Young

$\boldsymbol{q}_{/ /}$e $\boldsymbol{q}_{\perp}$ coeficientes de stress ótico

$\Theta \quad$ sinal da lente térmica, $\theta$, normalizado pelo produto $P \alpha_{a b s} L_{e f}$

$\tau_{\boldsymbol{o}} \quad$ tempo de vida do nível emissor

$\tau_{\text {exp }} \quad$ tempo de vida experimental

$\tau_{R} \quad$ tempo de vida radiativo

$\tau_{n r} \quad$ tempo de vida não-radiativo

$\chi_{m} \quad$ susceptibilidade elétrica da matriz

$\chi_{e x} \quad$ susceptibilidade elétrica do estado excitado

$\chi_{g} \quad$ susceptibilidade elétrica do estado fundamental

$N_{\boldsymbol{e x}}$ densidade de população do estado excitado

$N_{g}$ densidade de população do estado fundamental

$N_{t} \quad$ densidade de população total

I intensidade do laser

$\boldsymbol{I}_{\boldsymbol{o}} \quad$ intensidade do laser na posição focal

I $\quad$ intensidade de saturação

$\sigma_{g} \quad$ seção de choque de absorção do estado fundamental

$\sigma_{e x}$ seção de choque de absorção do estado excitado

$\Delta \sigma$ diferença de seção de choque de absorção entre os estados excitado e fundamental

T transmitância

$\Delta T_{p v} \quad$ variação da transmitância entre o pico e o vale da curva z-scan

$\Delta Z_{p v}$ diferença entre a posição de pico e de vale da curva z-scan

$\Delta \phi_{o} \quad$ variação de fase induzida

$\Delta \phi_{L P}$ variação de fase devido ao efeito de lente de população

$\Delta \phi_{L T}$ variação de fase devido ao efeito de lente de térmica

$\boldsymbol{A}_{\boldsymbol{b}} \quad$ fator de abertura da íris

$S$ parâmetro de saturação 
f força do oscilador

$\delta \quad$ parâmetro de desintonia

$a_{o}$ coeficiente de atenuação no centro da linha

A parâmetro que relaciona as contribuições ressonante e não ressonante para a susceptibilidade 


\section{Lista de Figuras}

Figura 2-1 - Representações esquemáticas do calor gerado devido (a) impurezas, (b) defeito quântico e (c) transições não radiavas à partir do nível laser. Nesta figura as setas ascendentes denotam processos de excitação, as descendentes são processos de emissão radiativa e as onduladas representam processos de emissão não radiativas que geram calor.

Figura 2-2 - Perfil de temperatura, $\Delta T$, induzida pelo feixe de excitação, para diferentes tempos de exposição [2].

Figura 2-3 - Arranjo dos feixes de excitação e prova na configuração do experimento de LT com feixe duplo de modo descasado, onde $L 1$ e $L 2$ são as lentes e $w_{e}$ e $w_{o p}$ os raios dos feixes de excitação e prova respectivamente.

Figura 2-4 -Comportamento transiente da intensidade do sinal de LT. (a) meio com $d s / d T>0$, ou seja, gerador de uma lente positiva; (b) meio com $d s / d T<0$, gerador de uma lente negativa.

Figura 2-5 -Aparato experimental do experimento de LT. Onde 1 é o laser de excitação, 2 é o laser de prova, 3 é o chopper, 4 são os espelhos, 5 as lentes, 6 a amostra, 7 os detectores, 8 a íris, 9 o osciloscópio e 10 o computador de aquisição de dados.

Figura 2-6 - Espectro de absorção do vidro germanato dopado com $\mathrm{Yb}^{3+}$. O gap do material está em torno de $600 \mathrm{~nm}$. $\mathrm{L}=1 \mathrm{~mm}$.

Figura 2-7 - (a) Sinal transiente ajustado com a Equação 2-5, para $P=20 \mathrm{~mW}$ e $\lambda=975 \mathrm{~nm}$. (b) Sinal de LT normalizado pela absorção e comprimento efetivo da amostra como função da potência incidente, para o vidro germanato. $\mathrm{L}=1 \mathrm{~mm}$.

Figura 2-8 - Comportamento transiente do sinal de LT no vidro QX/Yb. Para $P=164 \mathrm{~mW}$ e $\lambda=975 \mathrm{~nm}$ foram obtidos $\theta=-0.0452 \mathrm{rad}$ e $t_{c}=1,6 \mathrm{~ms}$.

Figura 2-9 - (a) espectro térmico da amostra QX/Yb. (b) espectro de absorção da amostra. Pode ser vista uma proporcionalidade entre estes espectros. As linhas são apenas para guiar os olhos.

Figura 2-10 -Sinal da LT normalizado pela potência absorvida como função do comprimento de onda de bombeamento. Do ajuste linear foram obtidos $\eta=(0.92 \pm 0.08)$ e $C=(3.3 \pm 0.2) \mathrm{W}^{-1}$. Na parte superior temos o espectro de absorção, para comparação.

Figura 2-11 -Sinal transiente de LT para $P=81 \mathrm{~mW}$ e $\lambda=976 \mathrm{~nm}$ em uma amostra de 6 peso $\%$ de $\mathrm{Yb}^{3+}$. Do ajuste foram obtidos: $t_{c}=1,3 \mathrm{~ms}$ e $\theta=-0.07804 \mathrm{rad} . \mathrm{L}=2 \mathrm{~mm}$.

Figura 2-12 - Espectro do sinal de LT normalizado pela potência absorvida. Do ajuste linear foram obtidos $C=(9,7 \pm 0,8) \mathrm{W}^{-1}$ e $\eta=(0,92 \pm 0,15)$. 25

Figura 2-13 - Sinal de LT normalizado pela absorção e comprimento efetivo da amostra para o vidro aluminato. Os pontos experimentais foram ajustados com a Equação 2-7 
Figura 3-1 - Diagrama de níveis típico de um laser de estado sólido. As setas cheias indicam processos radiativos enquanto as tracejadas indicam processos não radiativos.

Figura 3-2 - Diagrama de construção da curva de z-scan. A parte superior da figura mostra o comportamento do feixe quando a amostra passa em torno do foco. A parte inferior da figura mostra a resposta da transmitância quando a amostra é deslocada. As linhas tracejadas indicam o caminho do feixe sem a amostra.

Figura 3-3 - Evolução temporal do sinal de $z$-scan da amostra de $\mathrm{GdAlO}_{3}: \mathrm{Cr}^{3+}\left(n_{2}>0\right)$, colocada em (a) $z=$ $0,85 z_{\mathrm{c}}(\mathrm{b}) \mathrm{z}=-0,85 \mathrm{z}_{\mathrm{c}}$ e (c) $z=7 \mathrm{z}_{\mathrm{c}}[16]$

Figura 3-4 - Aparato experimental utilizado nas medidas de Z-scan. 1 laser de excitação, 2 lentes, 3 chopper, 4 espelhos, 5 amostra, 6 estágio de translação, 7 divisor de feixes, 8 detectores, 9 íris, 10 amplificador, 11 osciloscópio e 12 computador de aquisição.

Figura 3-5 - Curva de z-scan para a fenda aberta em uma amostra de $\mathrm{Yb}^{3+}$ :fosfato de $8 \%$. Dados do experimento: $P=100 \mathrm{~mW}, \alpha_{a b s}=7.8 \mathrm{~cm}^{-1}, L=1.77 \mathrm{~mm}, \omega_{0}=29 \mu \mathrm{m}$. Os círculos cheios foram obtidos para o detector com uma íris de $50 \%$ de transmitância.

Figura 3-6 - Curva de z-scan, para a fenda fechada, já normalizada pela curva da fenda aberta. Aqui $P=100 \mathrm{~mW}$.

Figura 4-1 -Perfil radial do índice de refração, $n(r)$ para diferentes parâmetros de saturação, $S=I / I_{S}$. O efeito da saturação no centro do feixe torna o perfil de n(r) achatado. 56

Figura 4-2 -Sinal transiente transmitido pela amostra de rubi, para duas diferentes potências. Para $\mathrm{P}=9 \mathrm{~mW}$ foi obtido $\tau=2,31 \mathrm{~ms}$ e para $\mathrm{P}=4 \mathrm{~mW}$ foi obtido $\tau=2,98 \mathrm{~ms}$.

Figura 4-3 - Inverso do tempo de respostada não linearidade como função da intensidade incidente. Neste gráfico temos $\lambda=528 \mathrm{~nm}, \tau_{0}=(3,4 \pm 0,2) \mathrm{ms} \mathrm{e} \mathrm{I}_{\mathrm{S}}=(1,0 \pm 0,1) \mathrm{KW} / \mathrm{cm}^{2}$, onde os valores para $\tau_{0}$ e $\mathrm{I}_{\mathrm{S}}$ foram obtidos à partir da Equação 4-5.

Figura 4-4 - Comportamento de $I_{S}{ }^{*} / I_{S}$ em função de $\lambda$. este comportamento é proporcional à $\eta_{\mathrm{p}}$. A linha cheia é um guia para os olhos.

Figura 4-5 - Curvas normalizadas do tempo de resposta como função do parâmetro de saturação, para os comprimentos de onda estudados.

Figura 4-6 -Decaimento temporal da luz transmitida pela amostra. (a) resultado experimental e (b) simulação numérica. Dados experimentais para $\lambda=528 \mathrm{~nm}$.

Figura 4-7 - Curvas teóricas de z-scan obtidas através da integral de difração. Nesta figura percebe-se um ligeiro aumento de $\Delta \mathrm{z}_{\mathrm{pv}}$ quando $\mathrm{S}$ aumenta. 
Figura 4-8 - Efeito do alargamento de $\Delta \mathrm{z}_{\mathrm{pv}}$ previsto das simulações numéricas. Estes dados foram obtidos da Figura 4-7. Aqui $\mathrm{z}_{\mathrm{c}}=1 \mathrm{~cm}$.

Figura 4-9 - Curvas de z-scan saturadas em $\lambda=528 \mathrm{~nm}$. Note que existe um leve alargamento das curvas com o aumento da potência incidente.

Figura 4-10 - Comportamento experimental da distância de pico a vale da curva de Z-scan como função do parâmetro de saturação, $\mathrm{S}$, para $\lambda=528 \mathrm{~nm}, \mathrm{I}_{\mathrm{S}}=1 \mathrm{KW} / \mathrm{cm}^{2}$ e $\mathrm{z}_{\mathrm{c}}=0,81 \mathrm{~cm}$. os dados experimentais foram ajustados com a Equação 4-6.

Figura 4-11 - Círculos: curva de z-scan saturada em rubi, onde $\mathrm{S}=0,5$ e. $\lambda=501 \mathrm{~nm}$. Linha cheia: simulação numérica da mesma medida, mas desprezando o efeito da saturação.

Figura 4-12 - Variação da transmitância entre o pico e o vale da curva de z-scan (fenda fechada) num cristal de rubi como função da intensidade incidente, para os comprimentos de onda do laser de $\mathrm{Ar}^{+}$. As linhas cheias representam ajustes feitos com a Equação 4-2.

Figura 4-13 - Mudança no caráter da absorção de saturável $(501 \mathrm{~nm})$ para saturável reverso $(457 \mathrm{~nm})$ num cristal de rubi com o aumento da energia de excitação.

Figura 4-14 - Máxima variação da transmitância para a fenda aberta num cristal de rubi como função da intensidade incidente. As linhas cheias são ajuste feitos com a Equação 4-3. Acima da linha tracejada estão os casos que apresentaram absorção saturada e abaixo os que apresentaram absorção saturada reversa.

Figura 5-1 - Diagrama esquemático do processo de upconversion Auger. (a) Inicialmente dois íons no estado excitado interagem não radiativamente; (b) logo após um dele é levado a um estado excitado superior enquanto o outro vai para um estado excitado inferior. As setas onduladas indicam processos não radiativos.

Figura 5-2 - Diagrama para excitação não ressonante ao nível emissor para uma amostra dopada com $\mathrm{Nd}^{3+}: \mathrm{YAG}$.

Figura 5-3 - Simulação da fração de população do estado excitado em função do parâmetro de saturação $S$, para vários valores do parâmetro de perda $\beta$. 82

Figura 5-4 - Comportamento transiente da fluorescência do nível laser para diferentes valores do parâmetro $\beta$.

Figura 5-5 - Decaimentos obtidos para excitação baixa $\left(\mathrm{n}_{\mathrm{ex}}(0)<10^{-2}\right)$, curva mais alta, e para alta excitação $\left(\mathrm{n}_{\mathrm{ex}}(0) \sim 0,2\right.$ e 0,9$)$, curva mais baixa, em amostras de $\mathrm{Nd}^{3+}$ para diferentes concentrações. 84

Figura 5-6 - (a) amplitude do sinal de LT; (b) $\theta$ normalizado pela potência absorvida e (c) comportamento da eficiência quântica como função de $\mathrm{S}$ para o cristla de $\mathrm{Cr}^{3+}$ :LiSAF com dopagens de 1\% (círculos fechados) e 7\% (círculos abertos). 
Figura 5-7 - Variação da transmitância para o cristal de YAG dopado com $\mathrm{Nd}^{3+}$. Usando $\lambda=808 \mathrm{~nm}$, $\Delta \sigma=2,8 \times 10^{-20} \mathrm{~cm}^{2}$, e $N_{t}=6,1 \times 10^{19} \mathrm{~cm}^{-3}$ foram obtidos $\beta=(2,1 \pm 0,5)$ e $\mathrm{I}_{\mathrm{S}}=(15 \pm 1) \mathrm{KW} / \mathrm{cm}^{2}$ usando a Equação 5-10 para o ajuste. $\mathrm{O}$ caso $\beta=0$ também é mostrado para comparação.

Figura 5-8 -Variação da transmitância para o cristal de YLF dopado com $\mathrm{Nd}^{3+}$. Usando $\lambda=792 \mathrm{~nm}$, foram obtidos $\beta=(11 \pm 2)$ e $\mathrm{I}_{\mathrm{S}}=(25 \pm 2) \mathrm{KW} / \mathrm{cm}^{2}$.

Figura 5-9 - Variação da transmitância para o cristal de $\mathrm{YVO}_{4}$ dopado com $\mathrm{Nd}^{3+}$. Usando $\lambda=808 \mathrm{~nm}$, foram obtidos $\beta=(4,6 \pm 2,3)$ e $\mathrm{I}_{\mathrm{S}}=(13 \pm 3) \mathrm{KW} / \mathrm{cm}^{2}$.

Figura 5-10 - Variação da transmitância para o cristal de LiSAF dopado com $\mathrm{Cr}^{3+}$. Usando $\lambda=808$ $\mathrm{nm}$, foram obtidos $\beta=(61 \pm 18)$ e $\mathrm{I}_{\mathrm{S}}=(904 \pm 124) \mathrm{KW} / \mathrm{cm}^{2}$. 90

Figura 5-11 -Diagrama de níveis de energia do $\mathrm{Nd}^{3+}$ no cristal de YAG para o caso de excitação ressonante, mostrando seus níveis e subníveis de interesse. 93

Figura 5-12 - Variação da transmitância num cristal de YAG dopado com $\mathrm{Nd}^{3+}$ sob excitação ressonante. No ajuste foram usados $\lambda=869 \mathrm{~nm}, f_{21}=0.6$ e $f_{11}=0.462$ e foram obtidos $\beta=(3 \pm 2)$ e $\mathrm{I}_{\mathrm{S}}=$ $(87 \pm 4) \mathrm{KW} / \mathrm{cm}^{2}$. A linha cheia é o ajuste feito com a expressão para $\Delta T$ usando agora a Equação 5-18 para $N_{e x}$.

96

Figura 5-13 - Variação da transmitância num cristal de YLF dopado com $\mathrm{Nd}^{3+}$ sob excitação ressonante. No ajuste foram usados $\lambda=862 \mathrm{~nm} f_{21}=0,57$ e $f_{11}=0,429$ e foram obtidos $\beta=(7,5 \pm 3)$ e $\mathrm{I}_{\mathrm{S}}$ $=(67 \pm 4) \mathrm{KW} / \mathrm{cm}^{2}$.

Figura 5-14 - Variação da transmitância num cristal de $\mathrm{YVO}_{4}$ dopado com $\mathrm{Nd}^{3+}$ sob excitação ressonante. No ajuste foram usados $\lambda=879 \mathrm{~nm} f_{21}=0,52$ e $f_{11}=0,4$ e foram obtidos $\beta=(14 \pm 5)$ e $\mathrm{I}_{\mathrm{S}}=$ $(24 \pm 3) \mathrm{KW} / \mathrm{cm}^{2}$.

Figura 5-15 - Variação na transmitância entre pico e vale da curva de z-scan com a fenda fechada. Os círculos com barras de erro denotam os pontos experimentais, as demais curvas foram obtidas através de simulações numéricas. Usando $L_{e f}=0.1 \mathrm{~cm}, \mathrm{n}_{2}{ }^{\prime}=5,6 \times 10^{-9} \mathrm{~cm}^{2} / \mathrm{W}$ e $\mathrm{I}_{\mathrm{s}}=18 \mathrm{KW} / \mathrm{cm}^{2}$ obtivemos a melhor concordância para $\beta=6$.

Figura 5-16 - Comportamento da fração de população do estado excitado para (a) valores diferentes de $\beta$ fixo e valores diferentes de $S$ e (b) $S$ fixo e valores diferentes de $\beta$. 103

Figura 5-17 - Comportamento transiente do sinal transmitido da fenda aberta ajustado para um cristal de $\mathrm{Nd}^{3+}$ :YAG bombeado em $808 \mathrm{~nm}$ e uma concentração de $0,75 \%$, com as Equação 5-23. Neste ajuste $\tau_{0}=240 \mu \mathrm{s}$ e $\mathrm{S}=0,5$. 104

Figura 5-18 - Comportamento do tempo característico da não linearidade como função do parâmetro de saturação, $S$, para diferentes valores de $\beta$. 105 
Figura 5-19 -Comportamento do tempo característico da não linearidade como função de $\mathrm{S}$ para a fenda aberta. No mesmo gráfico vemos o caso $\beta=0$.

Figura 6-1 - Forma de linha absortiva (linha cheia) e dispersiva (tracejada) na vizinhança de uma absorção. A escala vertical serve tanto para absorção como para o índice de refração.

Figura 6-2 - Mudança de fase numa fibra de sílica dopada com $\mathrm{Yb}^{3+}$, em unidades de $\pi$. A fibra foi excitada em $980 \mathrm{~nm}$ e provada em diversos comprimentos de onda

Figura 6-3 - Aparato experimental usado para medidas de Z-scan e espectroscopia. $\mathrm{M}=$ espelho, $\mathrm{L}=$ lente, $\mathrm{BS}=$ divisor de feixe, $\mathrm{D}=$ detector, $\mathrm{S}_{1}$ e $\mathrm{S}_{2}$ as transmitâncias da íris à frente dos detectores, Ref é o sinal de referência usado para obter a transmitância linear da amostra.

Figura 6-4 - Determinação dos parâmetros $I_{S}$ e $\tau_{0}$ através da técnica de refração transiente. (a) Sinal transiente transmitido pela amostra. (b) Inverso do tempo de resposta da não linearidade como função da intensidade incidente. Do ajuste foram obtidos: $\mathrm{I}_{\mathrm{S}}=4,3 \mathrm{KW} / \mathrm{cm}^{2}$ e $\tau_{0}=2,4 \mathrm{~ms}$.

Figura 6-5 - Medidas de z-scan no vidro $\mathrm{QX} / \mathrm{Yb}$ para $\lambda$ no pico da linha de absorção. (a) Curva de saturação, $A_{b}=100 \%$ e (b) curva de refração $A_{b}=50 \% / A_{b}=100 \%$.

Figura 6-6 - Espectro não linear do $\mathrm{Yb}^{3+}$ no vidro QX obtido com medidas convencionais de z-scan. Na parte superior pode-se ver o espectro de absorção linear, para comparação.

Figura 6-7- Diferença de polarizabilidade como função do comprimento de onda incidente. 122

Figura 6-8 - Espectro não linear do $\mathrm{Yb}^{3+}$ em torno do pico de absorção. 123

Figura 6-9 - Simulação da dependência da parte real do índice de refração não-linear, $n_{2}$ ', em função de $\delta$ para diferentes valores de $A$. 


\section{Lista de tabelas}

Tabela 2-1 -Parâmetros fototérmicos para diversos materiais laser, obtidos na literatura.

Tabela 3-1 -Parâmetros não lineares de sólidos dopados com $\mathrm{Nd}^{3+}$ e $\mathrm{Yb}^{3+}$ obtidos com a técnica de $\mathrm{z}$ scan. São mostrados: $\lambda(\mathrm{nm}), \tau(\mu \mathrm{s}), n_{2}{ }^{\prime}\left(10^{-10} \mathrm{~cm}^{2} \mathrm{~W}^{-1}\right), n_{2} ”\left(10^{-12} \mathrm{~cm}^{2} \mathrm{~W}^{-1}\right), \Delta \sigma\left(10^{-21} \mathrm{~cm}^{2}\right)$ e $\Delta \alpha\left(10^{-26}\right.$ $\left.\mathrm{cm}^{3}\right)$.

Tabela 3-2 -Distâncias focais devidas às lentes térmica e de população, em materiais dopados com $\mathrm{Nd}^{3+}$ e $\mathrm{Yb}^{3+}$.

Tabela 4-1 -Tempo de vida e intensidade de saturação e coeficiente de absorção para os comprimentos de onda do $\mathrm{Ar}^{+}$. $\mathrm{I}_{\mathrm{S}}{ }^{*}$ é o valor calculado de $\mathrm{I}_{\mathrm{S}}$.

Tabela 4-2 - Parâmetros não lineares obtidos à partir das medidas saturadas de z-scan. 72

Tabela 5-1 - Parâmetros usados nos ajustes: $\lambda(\mathrm{nm}), \tau(\mu \mathrm{s}), \mathrm{I}_{\mathrm{S}}^{*}\left(\mathrm{KW} / \mathrm{cm}^{2}\right)$ calculado, $\mathrm{I}_{\mathrm{S}}\left(\mathrm{KW} / \mathrm{cm}^{2}\right)$ obtido.

Tabela 5-2 - Parâmetros obtidos das medidas de Z-scan resolvida no tempo: $\lambda(\mathrm{nm}), \mathrm{n}_{2}$ " (x10 $0^{-10}$ $\left.\mathrm{cm}^{2} / \mathrm{W}\right), \Delta \sigma\left(\times 10^{-20} \mathrm{~cm}^{2}\right), \mathrm{N}_{\mathrm{t}}\left(\times 10^{20} \mathrm{~cm}^{-3}\right), \gamma\left(\times 10^{-16} \mathrm{~cm}^{3} / \mathrm{s}\right)$

Tabela 5-3 -Parâmetros usados nos ajustes: $\lambda(\mathrm{nm}), \tau(\mu \mathrm{s}), \mathrm{I}_{\mathrm{S}} *\left(\mathrm{KW} / \mathrm{cm}^{2}\right)$ calculado, $\mathrm{I}_{\mathrm{S}}\left(\mathrm{KW} / \mathrm{cm}^{2}\right)$ obtido.

Tabela 5-4 - Parâmetros obtidos das medidas de Z-scan resolvida no tempo: $\lambda(\mathrm{nm}), \mathrm{n}_{2}$ " (x10 $0^{-10}$ $\left.\mathrm{cm}^{2} / \mathrm{W}\right), \Delta \sigma\left(\times 10^{-20} \mathrm{~cm}^{2}\right), \mathrm{N}_{\mathrm{t}}\left(\mathrm{x} 10^{20} \mathrm{~cm}^{-3}\right), \gamma\left(\times 10^{-16} \mathrm{~cm}^{3} / \mathrm{s}\right), \mathrm{N}_{\mathrm{cr}}\left(\times 10^{19} \mathrm{~cm}^{-3}\right)$ 


\section{Resumo}

Neste trabalho estudamos as propriedades térmicas e óticas de íons emissores usando as técnicas de lente térmica e z-scan, resolvidas no tempo. Foram obtidos o espectro fototérmico de lente térmica de amostras dopadas com $\mathrm{Yb}^{3+}$ e diversos outros parâmetros fototérmicos. Foi também realizado um estudo dos efeitos da saturação da não linearidade ótica sobre a determinação das propriedades eletrônicas em sólidos dopados com $\mathrm{Cr}^{3+}$, utilizando a técnica de z-scan. A seguir a mesma técnica foi aplicada na obtenção do parâmetro de upconversion em materiais dopados com $\mathrm{Nd}^{3+}$ e $\mathrm{Cr}^{3+}$ (com excitação ressonante e não ressonante ao nível laser emissor). Empregando a integral de difração foram investigados os efeitos do processo de upconversion Auger sobre a refração não linear. Os resultados experimentais e numéricos mostraram uma excelente concordância. Também foi investigado o comportamento temporal da população do nível emissor na fase de excitação, demonstrando assim uma forma alternativa de obter o parâmetro de upconversion. Finalmente, usando a técnica de z-scan obtivemos a forma de linha da não linearidade ótica de uma amostra dopada com $\mathrm{Yb}^{3+}$ onde demonstramos que a contribuição mais importante para a não linearidade do íon advém de transições no UV, mesmo estando o íon sob excitação ressonante ao nível emissor. 


\begin{abstract}
In this work we have studied the thermal and optical properties of emitters ions by using the thermal lens and z-scan time resolved techniques. It was obtained the thermal lens photothermal spectra of $\mathrm{Yb}^{3+}$ doped samples, and several other photothermal parameters. Also, it was done a study on the optical nonlinearity saturation effects over the determination of the optical properties in $\mathrm{Cr}^{3+}$ doped solids, using the z-scan technique. In the sequence the same technique was used in order to obtain the upconversion parameter in $\mathrm{Nd}^{3+}$ and $\mathrm{Cr}^{3+}$ doped materials (with excitation resonant and non resonant to the emitter level). Employing the diffraction integral, the effects of Auger upconversion process on the nonlinear refraction were investigated. The experimental and numerical results agree very well. It was also investigated the temporal behavior of the emitter level population during the excitation process, showing an alternative way to obtain the upconvertion parameter. Finally, by using the $\mathrm{z}$-scan technique we have obtained the optical nonlinear lineshape of a $\mathrm{Yb}^{3+}$ doped sample, and demonstrated that the major contribution to the optical non linearity arises from the UV transitions, even when the emitter level of the ion is under resonant excitation.
\end{abstract}




\section{Capítulo 1}

\subsection{Introdução Geral}

A ótica não linear tornou-se um importante subcampo da ótica desde seu surgimento, 45 anos atrás. Naquela época, as fontes de luz permitiam apenas o estudo de sistemas óticos lineares. Neste caso a polarização induzida no meio é proporcional ao campo elétrico aplicado. Contudo, com a introdução do laser em 1960 [1] a intensidade da luz propagando no material cresceu enormemente, de modo que a polarização deixa de ser proporcional ao campo incidente e os efeitos não lineares tornaram-se evidentes. Desde então uma grande variedade de efeitos não lineares foram observados, documentados e usados em numerosos dispositivos. Muitos destes fenômenos interessantes da ótica não linear estão relacionados com a variação do índice de refração. Esta variação tem permitido importantes aplicações tecnológicas, além de ser um ponto fundamental em diversas investigações científicas como holografia em tempo real e conjugação de fase [2-6].

Para materiais dopados com íons terras-raras e metais de transição as principais origens da variação no índice de refração são devidas ao efeito de redistribuição de população e ao efeito térmico, efeitos que aparecem ao se bombear o nível emissor. Estes materiais apresentam não linearidade ótica algumas ordens de grandeza maior que o material hospedeiro. Além disto, parte da energia absorvida por estes íons se transforma em calor, gerando lente térmica e outros efeitos indesejáveis no material. Isto influencia diretamente no comportamento do laser, alterando desde o perfil do feixe até a própria estabilidade da cavidade.

O bombeamento destes materiais provoca uma redistribuição da população entre os níveis do íon que está diretamente relacionada ao índice de refração não linear do íon dopante, $n_{2}$. Por exemplo, em lasers, as ondas estacionárias na cavidade produzem uma modulação espacial de intensidade 
gerando uma rede de índice de refração complexa, $\Delta n=n_{2} I$, onde a parte imaginária está relacionada ao ganho do material. As regiões da cavidade onde o campo elétrico se anula não produzem fótons no comprimento de onda do laser, de modo que apenas algumas regiões do meio ativo participam da geração de luz laser. Este efeito é conhecido na literatura como spatial hole burning. Na verdade a primeira observação de rede de índice de refração induzida por laser foi feita no rubi com o intuito de demonstrar de uma forma mais direta o efeito de spatial hole burning [7]. Vários estudos foram feitos relacionados a este tema: redes holográficas transientes, conjugação de fase por mistura de quatro ondas, mistura de duas ondas, biestabilidade ótica, biestabilidade em lasers, auto-focalização, modulação transversal de fase, entre outros (vide Ref [8,9] e referências dentro destas). Diversos tipos de experimentos foram feitos em fibras dopadas com terras raras (principalmente com $\mathrm{Nd}^{3+}$ e $\mathrm{Er}^{3+}$ ) onde se demonstrou aplicação das redes transientes como chaves óticas e também se estudou estas redes em meios amplificadores [10]. Por outro lado, vários grupos de pesquisa têm se dedicado ao desenvolvimento de lasers de alta potência usando cavidades com espelhos de conjugação de fase em meios amplificadores de estado sólido (Nd:YAG, Ti:safira, etc.) produzidos por mistura de quatro ondas $[4,5]$. Além disso, recentemente foi observado propagação de luz ultra-lenta e ultra-rápida em rubi e alexandrita, e o fenômeno foi atribuído a efeitos de $n_{2}$ [11-13].

Já o efeito térmico é responsável pelo aquecimento do meio laser. Isto acontece porque durante o processo de bombeamento óptico, nem toda a energia absorvida é convertida em luz. Uma fração desta energia é transformada em calor, fazendo com que o material laser seja aquecido. Esta fração de energia convertida em calor é denominada a carga térmica do material, $\varphi$. Inicialmente o aquecimento provoca uma degradação da qualidade do feixe, podendo, em casos extremos, chegar à fratura do material. $\mathrm{O}$ aquecimento, desta forma, determina a qualidade do feixe e a máxima potência de saída do laser. Vários são os mecanismos que geram calor, e entre eles podemos citar: o defeito quântico, i. e., a razão entre as energia do fóton gerado e a do fóton de bombeio, $h v / h v_{p}$; a absorção da radiação laser 
por impurezas no material laser; transições não radiativas a partir do nível laser superior (descrito pela eficiência quântica de fluorescência, que é a razão entre a taxa de decaimento radiativo e a taxa de decaimento total, que leva em conta os processos radiativos e não radiativos, a despopulação de um nível) e absorção de estado excitado seguida de relaxação não radiativa.Em materiais dopados com neodímio, por exemplo, a carga térmica é fortemente dependente do comprimento de onda de bombeio. Lasers baseados neste íon são tradicionalmente bombeados em $808 \mathrm{~nm}\left({ }^{4} F_{5 / 2}\right)$, introduzindo desta forma um defeito quântico parasítico entre o nível de bombeio e o nível laser emissor. Bombeamento ressonante ao nível ${ }^{4} \mathrm{~F}_{3 / 2}$ (em torno de $890 \mathrm{~nm}$ ) poderia, em princípio, levar a uma diminuição da carga térmica. Este esquema de bombeamento foi desconsiderado no passado por causa da baixa absorção da transição ${ }^{4} \mathrm{I}_{9 / 2} \rightarrow{ }^{4} \mathrm{~F}_{3 / 2}$ e também por causa da falta de lasers de diodo emitindo neste intervalo espectral. No entanto, o desenvolvimento recente de lasers de semicondutor nesta região espectral vem aumentando o interesse neste esquema de bombeamento. De fato, bombeamento ressonante já vem sendo utilizado para melhorar a performance de vários cristais dopados com $\mathrm{Nd}^{3+}$ [14-17]. Por outro lado, o uso de lasers de diodo como bombeamento produziu um aumento considerável na potência de sistemas laser. No caso dos lasers pulsados, com a diminuição do tempo de duração dos pulsos lasers a potência de pico aumentou consideravelmente, o que pode induzir dano no meio ativo. Do mesmo modo, os lasers que trabalham no regime de onda contínua passaram a alcançar dezenas de milhares de Watts [18, 19].

Como visto acima as contribuições térmica e eletrônica podem modificar profundamente o comportamento de dispositivos óticos que se utilizam de íons emissores. Portanto, estudar sua origem e magnitude é de fundamental importância no desenvolvimento/aperfeiçoamento destes dispositivos. Por isso, empregando as técnicas de Lente Térmica e Z-scan resolvidas no tempo, estudamos as contribuições térmica e eletrônica na variação do índice de refração em materiais dopados com $\mathrm{Nd}^{3+}$,

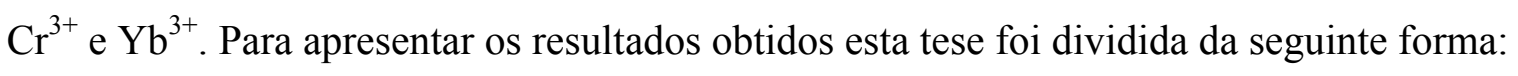


i) O Capítulo 1 faz uma introdução geral dos tópicos em estudo;

ii) O Capítulo 2 discute o efeito térmico em sólidos dopados e introduz a técnica de Lente Térmica para a determinação de suas propriedades fototérmicas. São também mostrados resultados de medidas em materiais dopados com $\mathrm{Yb}^{3+}$.

iii) O Capítulo 3 discute a origem da não linearidade eletrônica em sólidos dopados e introduz a técnica de z-scan para a determinação dos parâmetros não lineares relacionados à refração e absorção não linear.

iv) O Capítulo 4 discute o efeito da saturação sobre os dados experimentais de refração e a absorção não linear obtidos através da técnica de z-scan;

v) O Capítulo 5 trata das perdas por upconversion Auger. Estas perdas levam a uma diminuição na população do nível laser superior e ao mesmo tempo a um aumento na lente térmica. Neste capítulo utilizaremos a técnica de Z-scan para a determinação do parâmetro de upconversion, tanto para excitação ressonante como não ressonante do nível laser emissor. Assim como, introduziremos um método para obter o parâmetro de upconversion o comportamento transiente da população durante a fase de excitação $(I \neq 0)$.

vi) O Capítulo 6 apresenta medidas de espectroscopia eletrônica em íons dopados com $\mathrm{Yb}^{3+}$. Diversos parâmetros não lineares são obtidos destes espectros. Também aqui se discute a origem da não linearidade eletrônica em íons de $\mathrm{Yb}^{3+}$. Por fím se discute o peso das contribuições térmica e eletrônica na variação do índice de refração;

vii) Finalmente, o Capítulo 7 mostra as conclusões gerais da tese e apresenta as perspectivas de trabalhos ainda a serem desenvolvidos.

Resumindo, as principais contribuições desta tese foram o aperfeiçoamento da técnica de z-scan e sua utilização, conjuntamente com a técnica de Lente Térmica, em novos materiais. 


\subsection{Referências}

[1] T. H. Maiman, Stimulated Optical Radiation in Ruby, Nature 187 (1960) 493-494.

[2] T. Catunda and J. C. Castro, Phase Conjugation in GdAlO3-Cr3+ and Ruby, Opt. Comm. 63 (1987) 185-190.

[3] P. Roussignol, D. Ricard, J. Lukasik, and C. Flytzanis, New Results on Optical-Phase Conjugation in Semiconductor-Doped Glasses, J. Opt. Soc. Am. B 4 (1987) 5-13.

[4] O. L. Antipov, S. I. Belyaev, and A. S. Kuzhelev, Self-Pumped Phase-Conjugation of the Heterogeneous Light-Beam in the Inverted Nd-Yag-Rod with Nonreciprocal Feedback, Opt. Comm. 117 (1995) 290-294.

[5] A. Brignon and J. P. Huignard, Continuous-Wave Operation of Saturable-Gain Degenerate 4Wave-Mixing in a Nd-YVO4 Amplifier, Opt. Lett. 20 (1995) 2096-2098.

[6] P. C. de Souza, G. Nader, T. Catunda, M. Muramatsu, and R. J. Horowicz, Transient four-wave mixing in saturable media with a nonlinear refractive index, Opt. Comm. 163 (1999) 44-48.

[7] H. J. Eichler, P. Günter, and D. W. Pohl, Laser-Induced Dynamics Gratings, Springer-Verlag, New York, 1986.

[8] V. Pilla, P. R. Impinnisi, and T. Catunda, Measurement of saturation intensities in ion doped solids by transient nonlinear refraction, Appl. Phys. Lett. 70 (1997) 817-819.

[9] S. M. Lima, H. Jiao, L. A. O. Nunes, and T. Catunda, Nonlinear refraction spectroscopy in resonance with laser lines in solids, Opt. Lett. 27 (2002) 845-847.

[10] Y. C. Amado and P. T. Richard, Effect of Spectral Hole-Burning and Cross Relaxation on the Gain Saturation of Laser Amplifiers, J. App. Phys. 37 (1966) 3556-3563.

[11] M. S. Bigelow, N. N. Lepeshkin, and R. W. Boyd, Superluminal and slow light propagation in a room-temperature solid, Science 301 (2003) 200-202. 
[12] M. S. Bigelow, N. N. Lepeshkin, and R. W. Boyd, Observation of ultraslow light propagation in a ruby crystal at room temperature, Phys. Rev. Lett. 90 (2003) 113903.

[13] M. S. Bigelow, N. N. Lepeshkin, and R. W. Boyd, Ultra-slow and superluminal light propagation in solids at room temperature, J. Phys. Cond. Mat. 16 (2004) R1321-R1340.

[14] V. Lupei, A. Lupei, N. Pavel, T. Taira, I. Shoji, and A. Ikesue, Laser emission under resonant pump in the emitting level of concentrated Nd : YAG ceramics, Appl. Phys. Lett. 79 (2001) 590592.

[15] V. Lupei, G. Aka, and D. Vivien, Enhanced fundamental and self-frequency-doubling laser emission efficiency in F-4(3/2) directly pumped Nd-activated nonlinear crystals: The case of GdCa4O(BO3)(3), Appl. Phys. Lett. 81 (2002) 811-813.

[16] V. Lupei, N. Pavel, and T. Taira, Highly efficient continuous-wave 946-nm Nd : YAG laser emission under direct 885-nm pumping, Appl. Phys. Lett. 81 (2002) 2677-2679.

[17] Y. Sato, T. Taira, N. Pavel, and V. Lupei, Laser operation with near quantum-defect slope efficiency in Nd : YVO4 under direct pumping into the emitting level, Appl. Phys. Lett. 82 (2003) 844-846.

[18] Y. Jeong, J. K. Sahu, D. N. Payne, and J. Nilsson, Ytterbium-doped large-core fiber laser with 1.36 kW continuous-wave output power, Opt. Exp. 12 (2004) 6088-6092.

[19] Y. Jeong, J. K. Sahu, D. N. Payne, and J. Nilsson, Ytterbium-doped large-core fibre laser with 1 kW of continuous-wave output power, IEEE Elect. Lett. 40 (2004) 470-472. 


\section{Capítulo 2}

\section{Medidas de propriedades fototérmicas de vidros dopados com $\mathrm{Yb}^{3+}$}

\subsection{Efeito e a técnica de Lente Térmica}

O efeito de Lente Térmica (LT) é causado pela deposição de calor via processos de decaimento não radiativo após a energia do laser ter sido absorvida pela amostra. Em sólidos dopados, as impurezas (como os radicais de $\mathrm{OH}$ ) podem levar a um aumento significativo na quantidade de calor gerado [1]. Porém, este efeito pode ser minimizado, por exemplo, através de métodos mais eficientes de fabricação de amostras. No entanto, existem algumas fontes de geração de calor que são intrínsecas ao íon como o defeito quântico, que é a razão entre a energia média emitida radiativamente e a energia absorvida, $<h v_{\mathrm{em}}>/ h v_{\mathrm{ex}}$. Aqui o excesso de energia do processo excitação-emissão é transformado em calor. O próprio material hospedeiro (a matriz onde está inserido o íon) também pode influenciar o processo de geração de calor através da eficiência quântica de fluorescência, $\eta$ (definida como a razão entre a taxa de emissão radiativa e a taxa total de emissão de um dado nível, $\mathrm{W}_{\mathrm{R}} / \mathrm{W}_{\mathrm{T}}$ ), que depende de parâmetros da matriz como o índice de refração. A Figura 2-1 representa esquematicamente estas fontes de geração de calor.

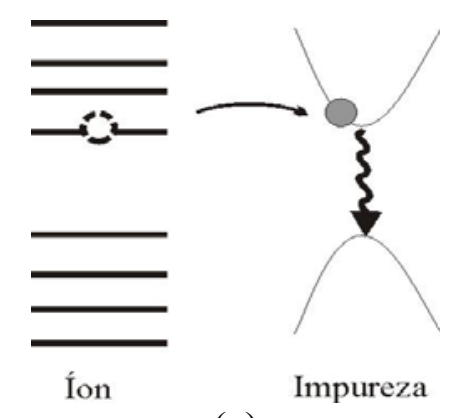

(a)

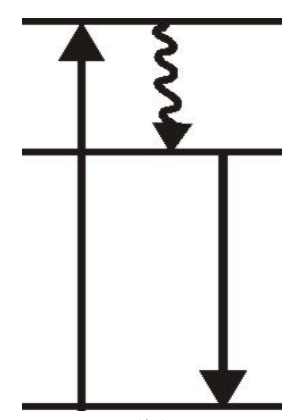

(b)

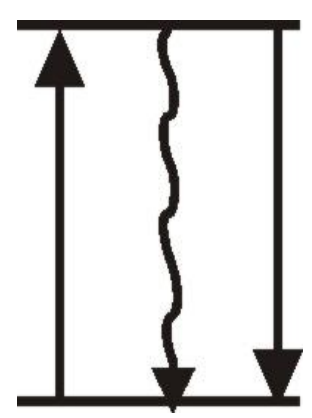

(c)

Figura 2-1 - Representações esquemáticas do calor gerado devido (a) impurezas, (b) defeito quântico e (c) transições não radiavas à partir do nível laser. Nesta figura as setas ascendentes denotam processos de excitação, as descendentes são processos de emissão radiativa e as onduladas representam processos de emissão não radiativas que geram calor. 
No caso de excitação com um feixe gaussiano, o calor se difunde na amostra de forma que no centro do feixe a variação de temperatura, $\Delta T(\mathrm{r}, \mathrm{t})$, é maior que na borda, conforme pode ser visto na Figura 2-2. Nesta figura $K$ é a condutividade térmica, $P$ a potência de excitação, $A$ o coeficiente de absorção, $w_{e}$ o raio do feixe de excitação na amostra e $t_{c}$ é um tempo característico da LT que é dado por:

$$
t_{c}=\frac{w_{e}{ }^{2}}{4 D}
$$

Aqui, $D$ é a difusividade térmica do material que está relacionada com a condutividade térmica por $K=\rho c_{p} D, \operatorname{com} \rho$ e $c_{\mathrm{p}}$ sendo a densidade de massa e o calor específico do material, respectivamente. Da Figura 2-2 nota-se que a temperatura aumenta com o tempo até que a quantidade de calor gerado na amostra pela excitação se iguale ao calor perdido para o ambiente devido à difusão térmica, alcançando assim o estado estacionário para $t \gg t_{c}$. Como um exemplo é possível estimar a variação de temperatura, $\Delta T$, no vidro fluoreto (no centro do feixe) sabendo que $K \sim 7 \times 10^{-3} \mathrm{~W} / \mathrm{Kcm}, A=1 \mathrm{~cm}^{-1}$ (em $\lambda \sim 500 \mathrm{~nm}$ ), para este vidro, e supondo uma potência de excitação de $100 \mathrm{~mW}$ para $t=25 t_{c}$, (vide Figura 2-2). Com estes dados o valor obtido foi de aproximadamente $\Delta T \sim 1,1^{\circ} \mathrm{C}^{1}$.

${ }^{1}$ No artigo original é dada a absorbância no lugar da absorção e a condutividade térmica é dada $\mathrm{em} \mathrm{Cal} \cdot \mathrm{s}^{-1} \cdot \mathrm{cm}^{-1} \cdot \mathrm{K}^{-1} \cdot \mathrm{A}$ Figura 2-2 está de acordo com a referência, embora o exemplo numérico esteja em concordância com as unidades usadas na tese. 


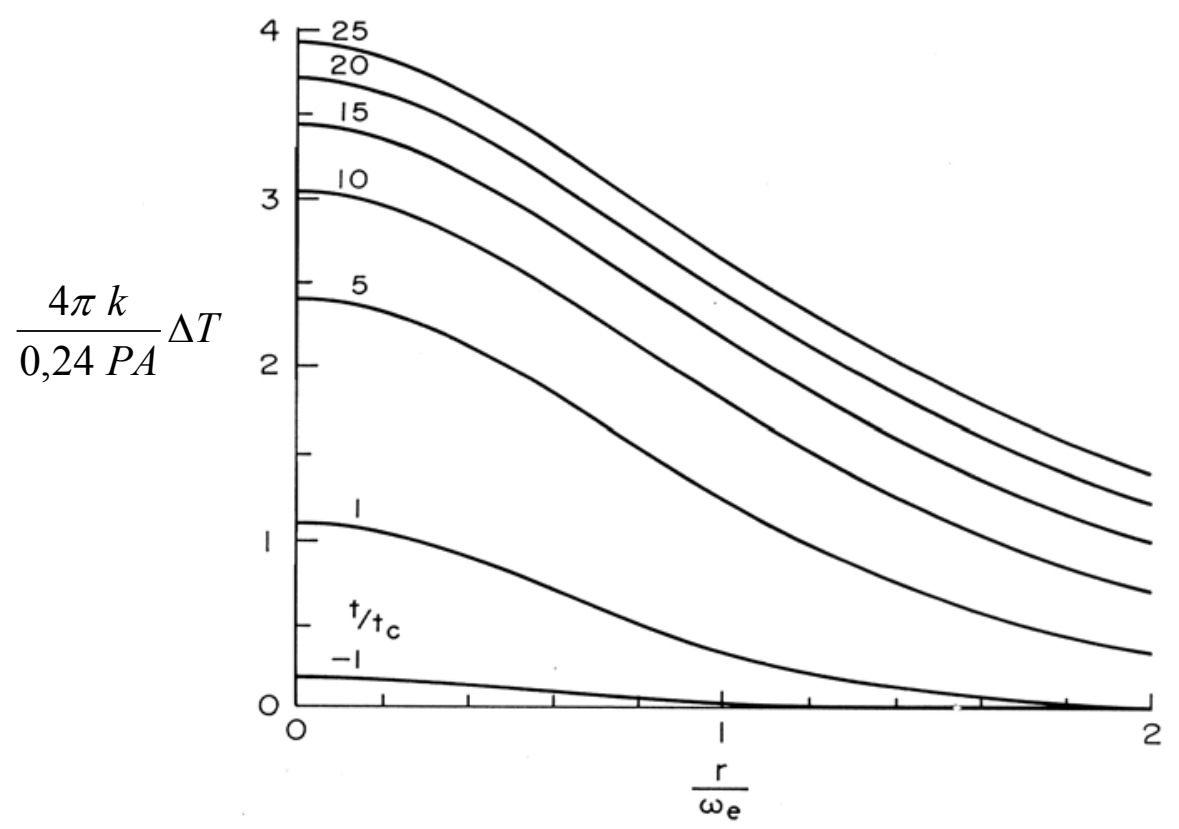

Figura 2-2 - Perfil de temperatura, $\Delta T$, induzida pelo feixe de excitação, para diferentes tempos de exposição [2].

Este perfil de temperatura gera uma variação térmica no índice de refração, $\Delta n_{T}$, que para líquidos pode ser escrita como:

$$
\Delta n_{T}=\frac{d n}{d T} \Delta T
$$

onde $d n / d T$ é o coeficiente termo-ótico do índice de refração. Como o perfil de $\Delta n_{T}$ segue o da temperatura, o meio se comporta como uma lente. Daí a designação: Lente Térmica. Esta LT pode ser tanto positiva/convergente como negativa/divergente dependendo do sinal de $d n / d T$. Na maioria dos líquidos $d n / d T$ deve-se a mudanças na densidade com a temperatura. Uma vez que a densidade decresce com o aumento da temperatura e o índice de refração é proporcional à densidade, então o coeficiente termo-ótico nestes meios é geralmente negativo. Em sólidos, o sinal de LT é proporcional ao coeficiente de variação do caminho ótico com a temperatura $d s / d T=(1 / L) d(n L) / d T$, que inclui não apenas a variação do índice de refração mas também do comprimento da amostra, $L$, devido a 
expansão térmica. Isto faz com que $d s / d T$ tenha três contribuições: $d n / d T$, expansão térmica e stress térmico. Logo, para geometria de disco fino (diâmetro >> L) chega-se à expressão [3-5]:

$$
\left.\frac{d s}{d T}\right|_{L T}=\frac{d n}{d T}+\alpha\left(n_{0}-1\right)(1+v)+\frac{n_{0}^{3}}{4} Y \alpha\left(q_{/ /}+q_{\perp}\right)
$$

onde $\alpha$ é o coeficiente linear de expansão térmica, $n_{0}$ é o índice de refração, $v_{p}$ é a razão de Poisson, $Y$ é o modulo de Young, $q_{/ /}$e $q_{\perp}$ são os coeficientes de stress ótico para as orientações paralela e perpendicular relativa à polarização do feixe incidente. O segundo termo da Equação 2-3 representa a curvatura das faces do material laser devido à maior expansão no centro, mais quente, comparada à borda, mais fria, da amostra, ou seja, o material expande mais no centro que na borda porque o feixe incidente é mais intenso no centro. Além disso, esta diferença de expansão provoca uma tensão entre estas regiões, i.e., stress térmico, que é expresso pelo terceiro termo na Equação 2-3.

\subsubsection{Técnica de LT}

O efeito de LT foi inicialmente descrito por Gordon et al em 1964 [6] para uma amostra líquida dentro de uma cavidade laser. A partir daí, alguns modelos foram propostos para quantificar a LT, entre eles podemos citar o modelo parabólico de Hu e Whinnery [7] e o modelo aberrante de Sheldon et al [2]. No modelo parabólico o perfil radial de temperatura, e consequentemente do índice de refração, são aproximados por uma parábola. Neste caso, $\Delta n_{T}(r)$ é equivalente a uma lente fina de distância focal $f_{\mathrm{LT}}$, a qual para uma amostra que não luminesce e no regime estacionário é dada por [7, 8]: 


$$
f_{L T}=\frac{\pi w_{e}^{2} K}{P_{a b s} d s / d T}
$$

Com $P_{a b s}=P \alpha_{a b s} L_{e f}$, onde $P$ é a potência do laser de excitação, $\alpha_{a b s}$ é o coeficiente de absorção óptica da amostra no comprimento de onda de excitação, $L_{e f}=\left(1-\mathrm{e}^{-\alpha_{a b s} L}\right) / \alpha_{a b s}$ é o comprimento efetivo da amostra e L a espessura da amostra.

Por outro lado, Sheldon et al [2] mostraram que a aproximação parabólica de Hu e Whinnery [7] fornecia resultados errados para $D$ e $d n / d T$ (fator de 50\%). Eles propuseram então uma abordagem baseada na integral de difração de Kirchhoff. Nesta aproximação eles usaram a integral para calcular o perfil do feixe no campo distante, o qual foi chamado aberrante, pois considera termos de ordem superior a $2 \mathrm{em}(\mathrm{r} / \mathrm{w})^{\mathrm{n}}$ como sendo equivalentes a aberrações óticas.

Entretanto o modelo mais geral, e mais sensível, para LT foi desenvolvido em 1992 por Shen et al [9], baseado no modelo aberrante de Sheldon et al [2]. Neste caso, a LT é gerada por um feixe de excitação, enquanto seu efeito é sentido por um feixe de prova que possui uma potência muito menor que o de excitação e, além disso, em geral está num comprimento de onda pouco absorvido pela amostra. Para facilitar a montagem experimental eles fazem um pequeno ângulo entre si, Figura 2-1.

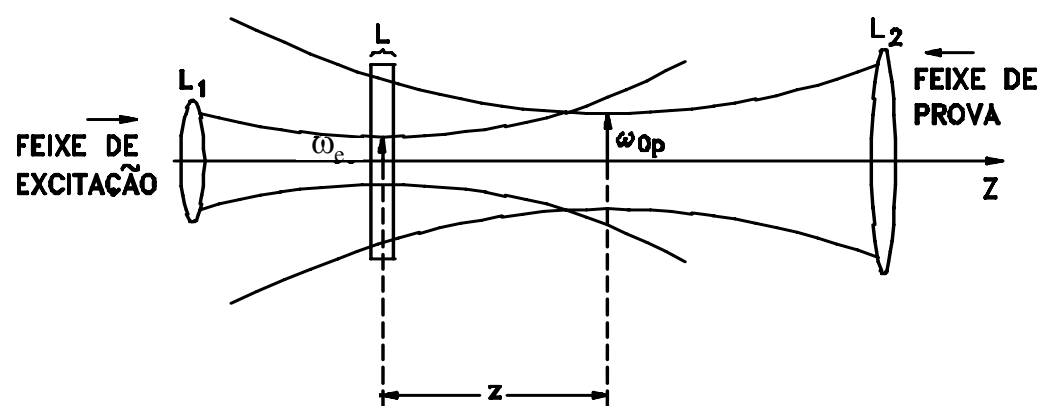

Figura 2-3 - Arranjo dos feixes de excitação e prova na configuração do experimento de LT com feixe duplo de modo descasado, onde $\mathrm{L}_{1}$ e $\mathrm{L}_{2}$ são as lentes e $w_{e}$ e $w_{o p}$ os raios dos feixes de excitação e prova respectivamente. 
Segundo o modelo de Shen a variação da intensidade no centro do feixe de prova no campo distante causado pela LT pode ser expressa, de acordo com a teoria de difração de Kirchhoff, da seguinte forma:

$$
I(t)=I(0)\left\{1-\frac{\theta}{2} \tan ^{-1}\left[\frac{2 m V}{\left[(1+2 m)^{2}+V^{2}\right] \frac{t_{c}}{2 t}+1+2 m+V^{2}}\right]\right\}^{2}
$$

onde $I(t)$ é o sinal transiente, $I(0)$ é o sinal transiente quando $t$ ou $\theta$ for zero, $m$ e $V$ são parâmetros geométricos da montagem da LT e são expressos por

$$
m=\left(\frac{w_{p}}{w_{o e}}\right)^{2} ; \quad V=\left(\frac{z}{z_{c p}}\right) \text { com } \mathrm{z}_{\mathrm{cp}} \ll \mathrm{z}_{2}
$$

onde $w_{\mathrm{p}}$ é o raio do feixe de prova na posição da amostra, $w_{o e}$ é a cintura mínima do feixe de excitação, z é a distância entre a cintura do feixe de prova e a amostra, $\mathrm{z}_{\mathrm{cp}}$ é o parâmetro confocal do feixe de prova e $z_{2}(\mathrm{~cm})$ é a distância da amostra ao detector que fará a leitura do sinal transiente. Normalmente a amostra é colocada na posição $\mathrm{z} \sim 1,7 \mathrm{z}_{\mathrm{cp}}$ que dá a máxima variação no sinal de LT. A amplitude de sinal transiente da LT $\theta$ é aproximadamente igual à diferença de fase do feixe de prova entre $r=0$ e $r=\sqrt{2} w_{o p}$ ( $w_{o p}$ é a cintura mínima do feixe de prova) induzida pela LT e é dada por:

$$
\theta=-\frac{P_{a b s}}{K \lambda_{p}} \phi \frac{d s}{d T}
$$

onde $\lambda_{p}$ é o comprimento de onda do laser de prova. O termo $\phi$, conhecido por carga térmica ou eficiência quântica não radiativa, representa a fração de energia que é transformada em calor. Para amostras não luminescentes toda energia absorvida é convertida em calor de modo que $\phi=1$. 
Entretanto, em amostras luminescentes, e com apenas um nível emissor, a carga térmica passa a ser dada por:

$$
\phi=1-\eta \frac{\left\langle v_{e m}\right\rangle}{v_{e x c}}=1-\eta\left\langle\lambda_{e m}^{-1}\right\rangle \lambda_{e x c}
$$

em que $v_{e x}(\mathrm{~Hz})$ a freqüência de excitação, $\left\langle v_{\mathrm{em}}\right\rangle$ é a freqüência média de emissão, $\lambda_{e x}(\mathrm{~nm}) \mathrm{o}$ comprimento de onda de excitação, $\eta$ a eficiência quântica da fluorescência e $\left\langle\lambda_{\mathrm{em}}^{-1}\right\rangle$ o valor médio do inverso do comprimento de onda da emissão, $\lambda_{e m}$. $\mathrm{O}$ valor de $\lambda_{e m}$ pode ser calculado a partir da probabilidade de emissão do nível laser para outros níveis, conhecida como razão de ramificação $\beta_{\mathrm{i}}$. Por exemplo, para o $\mathrm{Nd}^{3+}$ existem quatro possíveis caminhos de decaimento de ${ }^{4} \mathrm{~F}_{3 / 2} \rightarrow{ }^{4} \mathrm{I}_{\mathrm{J}}(\mathrm{J}=15 / 2$, 13/2, 11/2 e 9/2) com uma razão de ramificação $\beta_{\mathrm{i}}(\mathrm{i}=1,2,3$ e 4) para cada uma das transições. Assim, o comprimento médio de emissão pode ser calculado através de $\lambda_{e m}=\sum_{i} \beta_{i} \lambda_{i}$, onde $\beta_{\mathrm{i}}$ é calculado usando pelo método de Judd-Ofelt. ${ }^{2}$ Na Figura 2-4 vê-se o comportamento transiente do sinal de LT para duas amostras: uma com $d s / d T>0$, gerando uma lente positiva ou convergente, e outra com $d s / d T$ $<0$, gerando uma lente negativa ou divergente.

\footnotetext{
${ }^{2}$ Outra maneira de se calcular o comprimento médio de emissão é usar $\lambda_{e m}=\frac{\int \lambda I_{e m}(\lambda) d \lambda}{\int I_{e m}(\lambda) d \lambda}$, onde $I_{\mathrm{em}}(\lambda)$ é o espectro de
} emissão da amostra. 


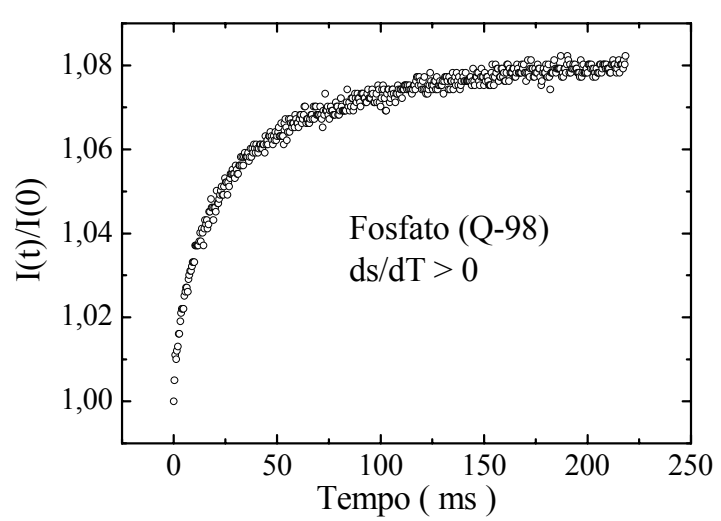

(a)

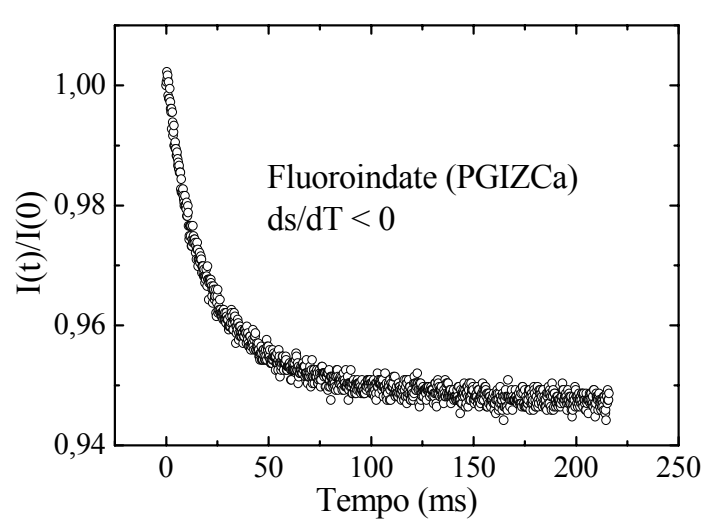

(b)

Figura 2-4 - Comportamento transiente da intensidade do sinal de LT. (a) meio com $d s / d T>0$, ou seja, gerador de uma lente positiva; (b) meio com $d s / d T<0$, gerador de uma lente negativa.

Numa análise através da técnica de LT, esse sinal é ajustado com a Equação 2-5 de onde se obtém os valores de $t_{c}$ e $\theta$. Usando então as Equações 2-1 e 2-7 é possível se calcular $D, K$ e $d s / d T$, uma vez que sejam conhecidos $\rho, c_{p}$ e $\phi$. Note que $d s / d T$ depende fortemente do índice de refração, Equação 2-3, de modo que o valor obtido por LT é válido apenas no comprimento de onda de prova, que foi o sinal detectado. Os demais resultados dependem apenas da estrutura do material estudado. A seguir apresentaremos os detalhes experimentais da montagem de LT.

\subsubsection{Arranjo experimental}

Na Figura 2-5 está esquematizada a montagem experimental para a LT na configuração de dois feixes no modo descasado. Nos experimentos citados neste trabalho utilizamos como laser de prova, um laser de $\mathrm{HeNe}$, operando em $632 \mathrm{~nm}$ com potência de $5 \mathrm{~mW}$ e como excitação um laser de $\mathrm{Ar}^{+}$ (457, 476 , 488 , 496 , 501 , 514 e $528 \mathrm{~nm})$, ou um laser sintonizável de Ti-safira (700 nm $<\lambda<1100$ nm). A montagem foi feita da seguinte forma: o feixe laser de excitação é conduzido até a amostra pelos espelhos sendo focalizado por uma lente convergente de foco $f=20 \mathrm{~cm}$. A amostra é posicionada na cintura do feixe de excitação, onde a intensidade é máxima, e ligeiramente deslocada da cintura do 
feixe de prova, Figura 2-3. O tempo de exposição da amostra ao feixe de excitação é controlado por um chopper cuja freqüência é variada de acordo com o tempo de resposta da amostra ( $t_{c}$, que depende de $w_{o e}$ ). Neste trabalho usamos freqüências em torno de $3 \mathrm{~Hz}$. O feixe de excitação, após passar pela amostra incide em um detector. Este sinal é utilizado para disparar o osciloscópio digital. O laser de HeNe é focalizado por uma lente convergente de foco maior que a do feixe de excitação, $f=30 \mathrm{~cm}$, e posicionada de forma que a suas posições focais fiquem espacialmente separadas (descasadas), Figura 2-3. O alinhamento é feito de maneira que o feixe de prova cruza com o de excitação com um ângulo de aproximadamente 1,5 graus. Para garantir a condição de detecção no campo distante, o feixe de prova percorre um longo caminho após passar pela amostra antes de chegar ao detector (em geral $\mathrm{z}_{2} \sim 1$ m) de forma que $z_{2}>z_{c p}$. À frente deste detector é então colocada uma íris de modo a permitir que apenas o centro do feixe do laser de prova seja monitorado. O sinal é armazenado por um osciloscópio digital Tektronix modelo TDS 210, que está conectado a um computador, onde é feita a análise dos dados. É importante lembrar que é necessário conhecer os valores da cintura mínima do feixe $w_{o}$ e de sua posição em relação à lente $z_{o}$, tanto para o feixe de prova como para o de excitação. O método usado para determinar $w_{o}$ e $z_{o}$ está mostrado no Apêndice C. 
(3)

(4)

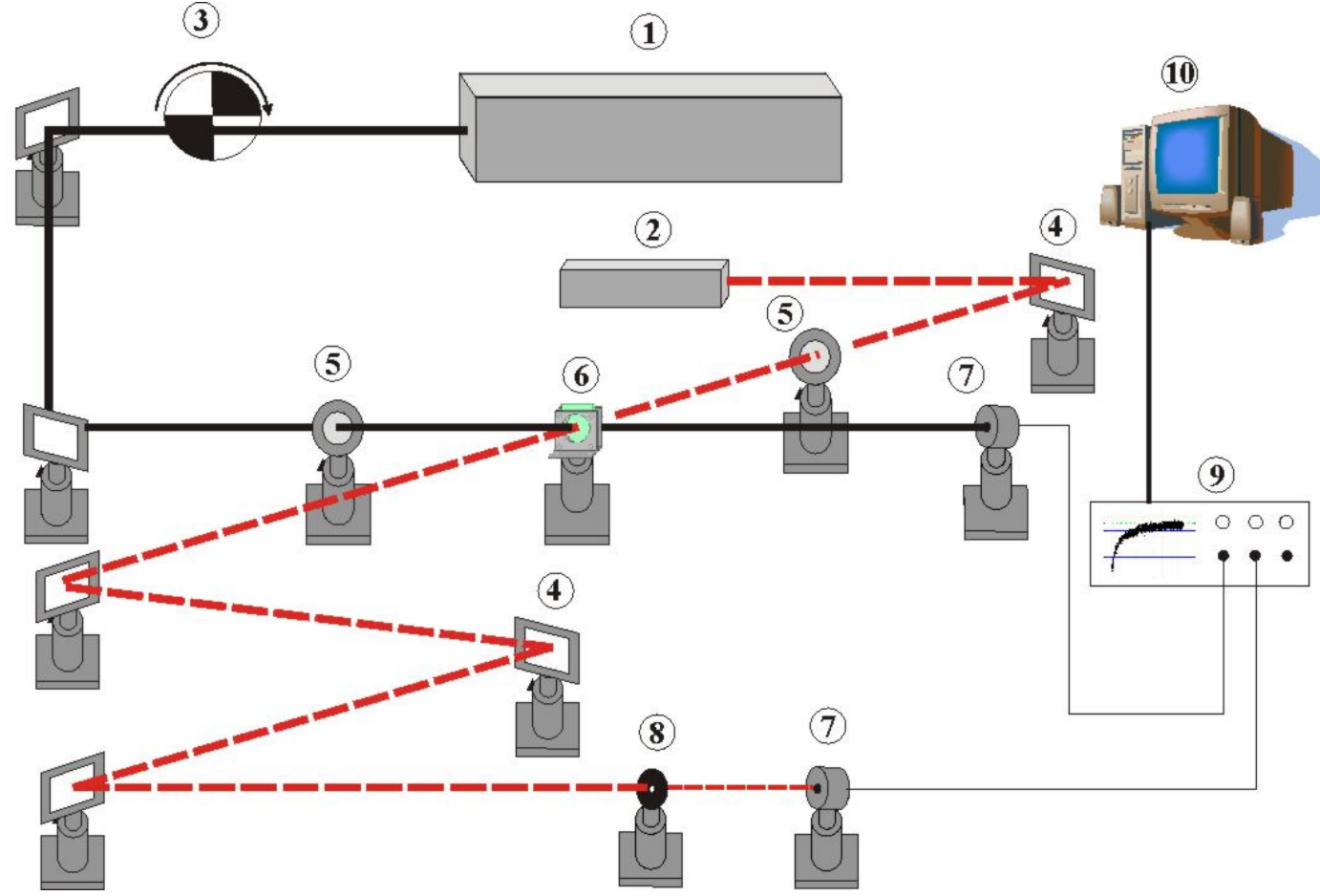

Figura 2-5 - Aparato experimental do experimento de LT. Onde 1 é o laser de excitação, 2 é o laser de prova, 3 é o chopper, 4 são os espelhos, 5 as lentes, 6 a amostra, 7 os detectores, 8 a íris, 9 o osciloscópio e 10 o computador de análise de dados.

Na subseção seguinte apresentamos alguns resultados obtidos para propriedades térmicas de materiais dopados com $\mathrm{Yb}^{3+}$ empregando a técnica de LT.

\subsubsection{Aplicação da técnica de LT a um vidro germanato dopado com $\mathrm{Yb}^{3+}$}

Os vidros semicondutores dopados com terras raras têm chamado muita atenção por causa das suas possíveis aplicações como fontes luminescentes com estreita largura de banda e termicamente estáveis $[10,11]$. Como se sabe, a estrutura eletrônica dos íons terras raras é dominada pelas interações elétronelétron e spin-órbita entre seus próprios níveis. Devido ao efeito de blindagem dos elétrons da camada $4 f$ pelas camadas mais externas, a matriz hospedeira normalmente não influencia muito na estrutura eletrônica do íon, mudando apenas a posição relativa dos níveis de energia. No entanto, no caso dos vidros semicondutores, as transições entre os níveis do íon terra rara sofrem influência do material 
hospedeiro devido ao entrelaçamento entre a banda do material e os níveis do íon. Diversos trabalhos já foram feitos no intuito de se estudar a interação entre as matrizes semicondutoras e alguns destes íons através de estudos de espectros de luminescência e de excitação [12-14]. Neste trabalho iniciamos o uso da técnica de LT para determinação dos parâmetros fototérmicos em um vidro germanato semicondutor dopados com $\mathrm{Yb}^{3+}$, excitando em $975 \mathrm{~nm}$. Existe ainda certa dificuldade na fabricação destes vidros visto que não é possível usar altas dopagens no material (ele rapidamente perde a qualidade ótica). No entanto, já é possível obter alguns resultados preliminares usando LT, como será apresentado a seguir.

A Figura 2-6 mostra o espectro de densidade ótica da amostra dopada com 0,5 massa\% de $\mathrm{Yb}^{3+}$. Note que o gap do material começa em torno de $600 \mathrm{~nm}(2,06 \mathrm{eV})$, o que está próximo dos valores encontrados para os vidros calcogenetos [15].

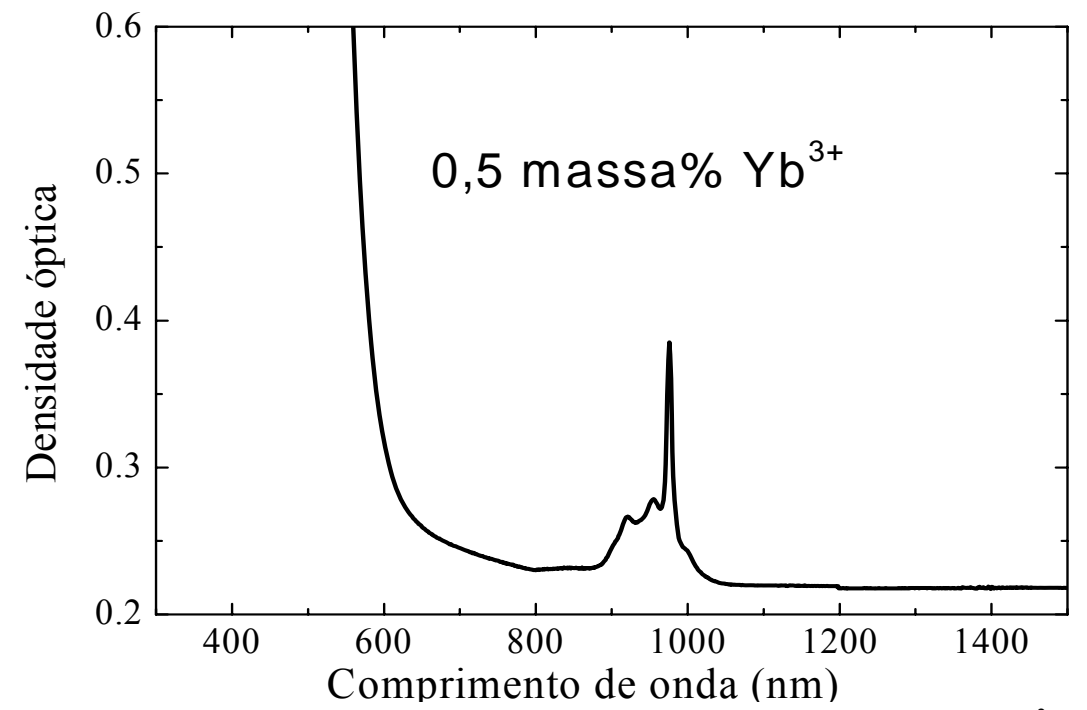

Figura 2-6 - Espectro de absorção do vidro germanato dopado com $\mathrm{Yb}^{3+}$. O gap do material está em torno de $600 \mathrm{~nm}$. L= $1 \mathrm{~mm}$.

A Figura 2-7(a) mostra um transiente típico obtido com o feixe de prova em $632,8 \mathrm{~nm}$ e para uma potência de excitação de $20 \mathrm{~mW}$.

\footnotetext{
${ }^{3}$ Leia-se percentagem em massa.
} 

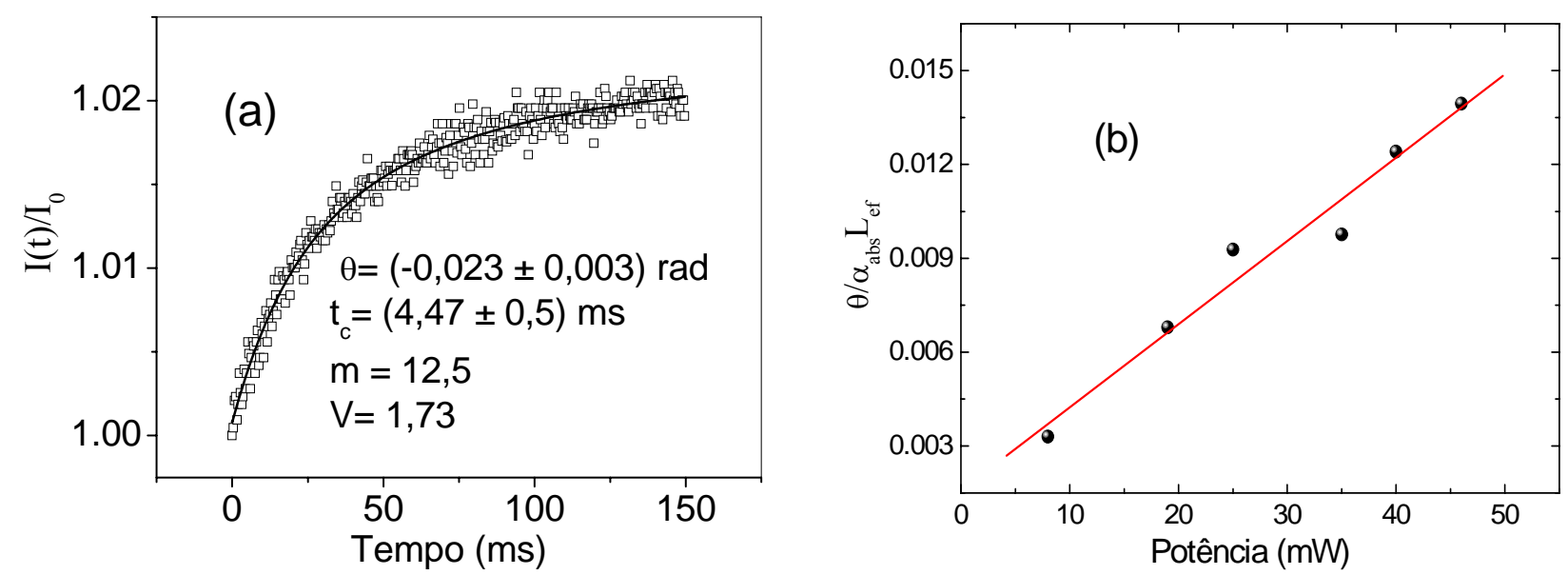

Figura 2-7 - (a) Sinal transiente ajustado com a Equação 2-5, para P $=20 \mathrm{~mW}$ e $\lambda=975 \mathrm{~nm}$. (b) Sinal de LT normalizado pela absorção e comprimento efetivo da amostra como função da potência incidente, para o vidro germanato dopado com itérbio. $\mathrm{L}=1 \mathrm{~mm}$.

O ajuste do sinal de LT foi feito com a Equação 2-5, onde $m$ e $V$ são mantidos fixos, e $\theta$ e $t_{c}$ são parâmetros ajustáveis. Através do ajuste do sinal transiente de LT foi encontrado $t_{c}=4,47 \mathrm{~ms}$, Figura 2-7(a), o que leva a. $D=(2,0 \pm 0,1) \times 10^{-3} \mathrm{~cm}^{2} / \mathrm{s}$ Da difusividade térmica, foi então calculada a condutividade térmica, $K$, como $K=(4,5 \pm 0,3) \times 10^{-3} \mathrm{~W} / \mathrm{Kcm}$, usando a relação $K=\rho c D$ e $\rho=7,2$ $\mathrm{g} / \mathrm{cm}^{3}$ e $c_{\mathrm{p}}=0,305 \mathrm{~J} / \mathrm{gK}$. Uma eficiência quântica de $\eta \sim 50 \%$ do $\mathrm{Yb}^{3+}$ na matriz germanato foi estimada a partir da razão entre o tempo de vida experimental, $\tau_{\exp } \sim 0,5 \mathrm{~ms}$, e o tempo de vida radiativo, $\tau_{\text {rad }} \sim 1 \mathrm{~ms}$, que foi obtido usando o método da reciprocidade, $c f$ Ref. [16] para detalhes. Ajustando então os dados da Figura 2-7(b) com a Equação 2-7, o valor de $K$ previamente obtido e $<\lambda_{\mathrm{em}}$ $>=1020 \mathrm{~nm}$ foi possível calcular $d s / d T=1,4 \times 10^{-6} / \mathrm{K} . \quad$ A Tabela 2-1 mostra alguns parâmetros fototérmicos de alguns vidros selecionados para comparação. Pode ser visto que os vidros germanatos $\mathrm{PbO}-\mathrm{Bi}_{2} \mathrm{O}_{3}-\mathrm{Ga}_{2} \mathrm{O}_{3}-\mathrm{BaO}$ apresentam pequeno valor de $d s / d T$ quando comparado com outros vidros. Isto é particularmente interessante na confecção de materiais atérmicos, $d s / d T=0$ [17], que são caracterizadas pela ausência de distorções óticas originadas da expansão térmica e a variação do índice 
de refração com a temperatura. Além disso, a condutividade e a difusividade térmica do vidro germanato são comparáveis ao vidro fosfato comercial LG750 e também ao vidro Ga:La:S. Com a evolução da técnica de fabricação do material será possível obter o espectro de LT e uma medida mais precisa da eficiência quântica de fluorescência, assim como estudar os efeitos gerados quando o bombeamento é feito na banda de absorção do material.

Recentemente, espectroscopia de LT foi utilizada em vidros calcogenetos (que também são vidros semicondutores) dopados com $\mathrm{Nd}^{3+}$ para determinação da eficiência quântica de fluorescência e a eficiência de transferência de energia entre a matriz e o íon de neodímio [15]. Com a evolução da técnica de fabricação do vidro germanato, esperamos que a qualidade ótica da amostra melhore a ponto de podermos também aplicar a técnica de LT para a determinação da eficiência de transferência de energia entre a matriz e o íon de $\mathrm{Yb}^{3+}$.

\section{2. $\quad$ Espectroscopia de $L T$ no vidro $Q X / Y b$}

Nossa amostra é um vidro fosfato com 1,4 $\mathrm{mm}$ de espessura, com uma dopagem de 6,8 massa\% de íons de $\mathrm{Yb}^{3+}\left(N_{t}=5,82 \times 10^{20}\right.$ íons $\left./ \mathrm{cm}^{3}\right)$. Este vidro é conhecido comercialmente como $\mathrm{QX} / \mathrm{Yb}$ e é manufaturado pela empresa Kigre Inc [18]. Neste vidro o comprimento de onda médio de emissão é $<\lambda_{\mathrm{em}}>=1004 \mathrm{~nm}$ [19], que está próximo ao pico de absorção, $975 \mathrm{~nm}$. Esta particularidade faz com que o defeito quântico seja muito pequeno, em torno de $3 \%$, de forma que os efeitos térmicos são bem menos pronunciados para este íon que para outros íons terras-raras. A amostra foi bombeada por um laser de Ti:safira sintonizado em torno do nível ${ }^{2} \mathrm{~F}_{5 / 2}$, que por sua vez foi bombeado por um laser de $\mathrm{Ar}^{+}$. A Figura 2-8 mostra o comportamento transiente do sinal de LT no pico de absorção em $975 \mathrm{~nm}$. Os pontos experimentais foram ajustados com a Equação 2-5. 


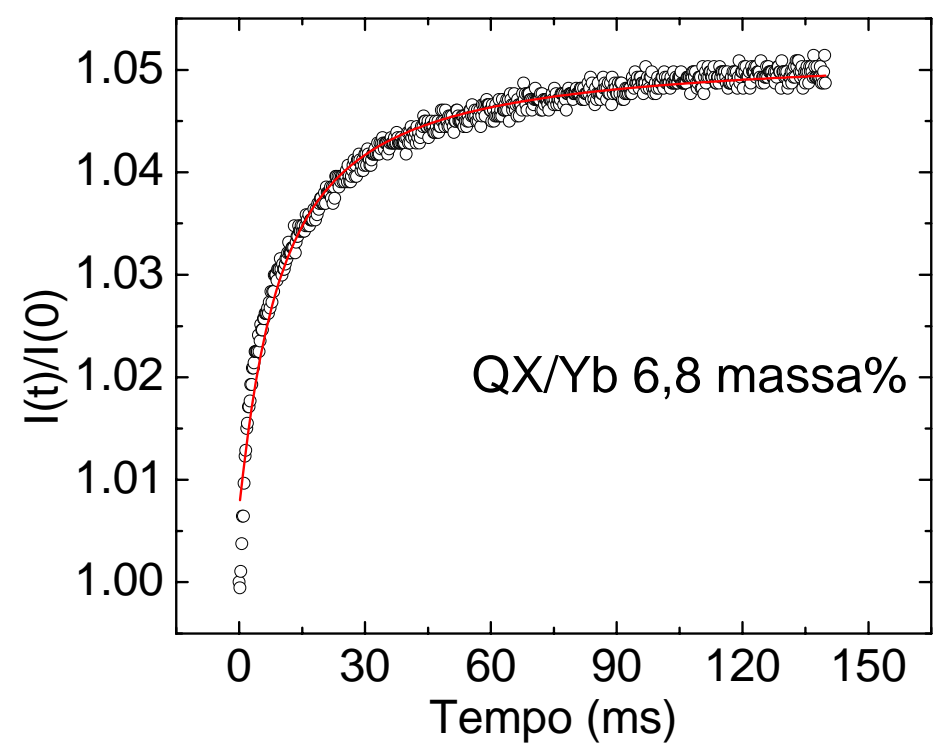

Figura 2-8 - Comportamento transiente do sinal de LT no vidro $\mathrm{QX} / \mathrm{Yb}$, $\mathrm{L}=1,4 \mathrm{~mm}$. Para $P=164 \mathrm{~mW}$ e $\lambda=975 \mathrm{~nm}$ foram obtidos $\theta=-0.0452$ $\operatorname{rad~e~} t_{c}=1,6 \mathrm{~ms}$.

Assim, para uma potência incidente de $164 \mathrm{~mW}$ foram obtidos $\theta=-0.0452 \mathrm{rad}$ e $t_{c}=1,6 \mathrm{~ms}$. A difusividade térmica, $D=(4,5 \pm 0,4) \times 10^{-3} \mathrm{~cm}^{2} / \mathrm{s}$, foi calculada, a partir de $t_{c}$, usando a Equação $2-1$. Através da relação $K=\rho c D$ a condutividade térmica foi calculada como $K=(10,1 \pm 0,9) \times 10^{-3} \mathrm{~W} / \mathrm{cmK}$, onde foram usados $\rho=2,81 \mathrm{~g} / \mathrm{cm}^{3}$ e $c=0,8 \mathrm{~J} / \mathrm{gK}$.

A Figura 2-9(a) mostra o sinal de LT normalizado pela potência incidente, $\theta / P$, como função do comprimento de onda de excitação. Este espectro é proporcional à fração de energia absorvida e transformada em calor e segue a absorção para cada comprimento de onda, como pode ser notado da Equação 2-7. Esta condição é confirmada observando a proporcionalidade entre os espectros de $\theta / P$ e $\alpha_{a b s} L_{e f}$ mostrados na Figura 2-9. Além disto, esta proporcionalidade indica que a eficiência quântica de fluorescência, $\eta$, é constante no intervalo de freqüência estudado. Este dado é importante pois nos permite utilizar a técnica de LT com múltiplos comprimentos de onda para a determinação de $\eta$. 


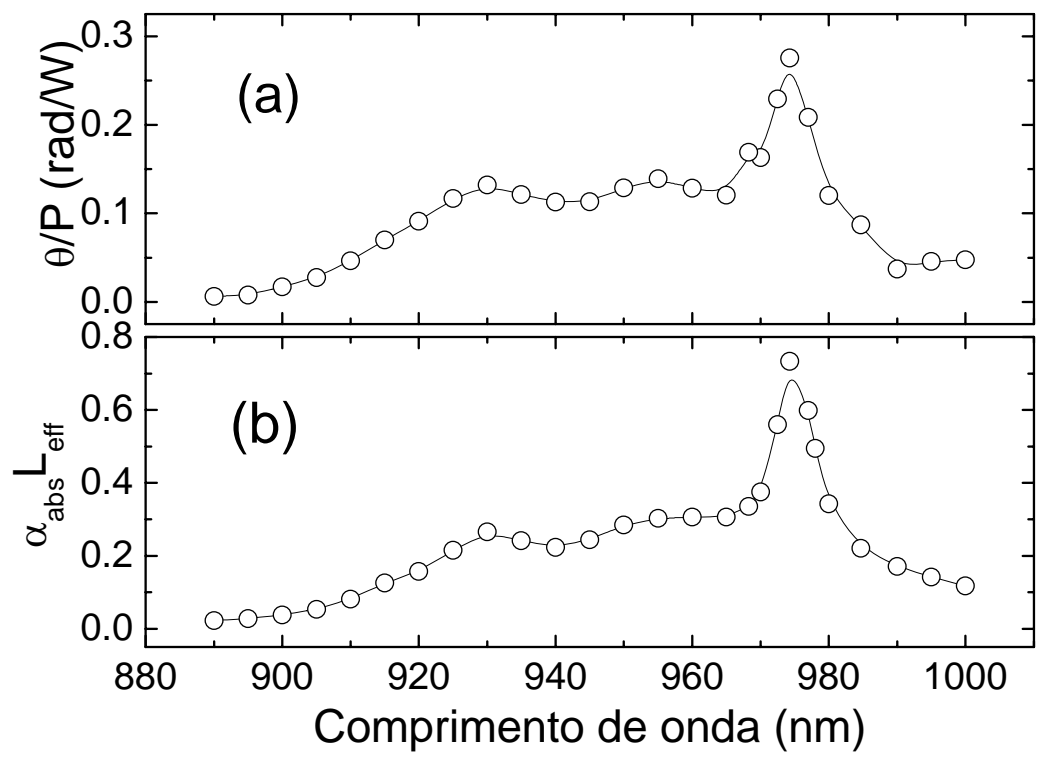

Figura 2-9 - (a) espectro térmico da amostra QX/Yb. (b) espectro de absorção da amostra. Pode ser vista uma proporcionalidade entre estes espectros. As linhas são apenas para guiar os olhos.

Este método foi introduzido por Lima et al [20], sendo então utilizado em diversos materiais dopados com $\mathrm{Nd}^{3+}[8,15,20-23]$ e está sendo agora, pela primeira vez (do nosso conhecimento), aplicado a um vidro comercial dopado com $\mathrm{Yb}^{3+}$. Isto pôde ser feito pois apesar de ter apenas um estado excitado acessível oticamente, este estado gera uma banda de absorção que se estende tipicamente por mais de 100 nm, Apêndice A. Para isto é conveniente re-escrever o sinal de LT como:

$$
-\frac{\theta}{P_{a b s}}=\Theta=\frac{1}{K \lambda_{p}} \frac{d s}{d T}\left(1-\eta \frac{\lambda}{\left\langle\lambda_{e m}\right\rangle}\right)
$$

$\mathrm{ou}$

$$
\Theta=C\left(1-\eta \frac{\lambda}{\left\langle\lambda_{e m}\right\rangle}\right)
$$

Assim, normalizando pela potência absorvida vemos que o sinal de LT mantém uma relação linear com comprimento de onda de excitação. Logo, uma vez conhecido $\left\langle\lambda_{\mathrm{em}}>\right.$, é possível, do ajuste 
linear de $\Theta \mathrm{x} \lambda$, obter a constante $C=\left(1 / K \lambda_{\mathrm{p}}\right) d s / d T$ (que depende de parâmetros da amostra e do comprimento de onda de prova) e a eficiência quântica de fluorescência, $\eta$. A Figura 2-10 mostra o ajuste linear de $\Theta \mathrm{x} \lambda$ para o vidro $\mathrm{QX} / \mathrm{Yb}$, onde deste ajuste foram obtidos $C=(3,3 \pm 0,2) \mathrm{W}^{-1} \mathrm{e}$ $\eta=(0,92 \pm 0,08) . \mathrm{O}$ erro dos pontos na Figura 2-10 foi estimado em aproximadamente $10 \%$.

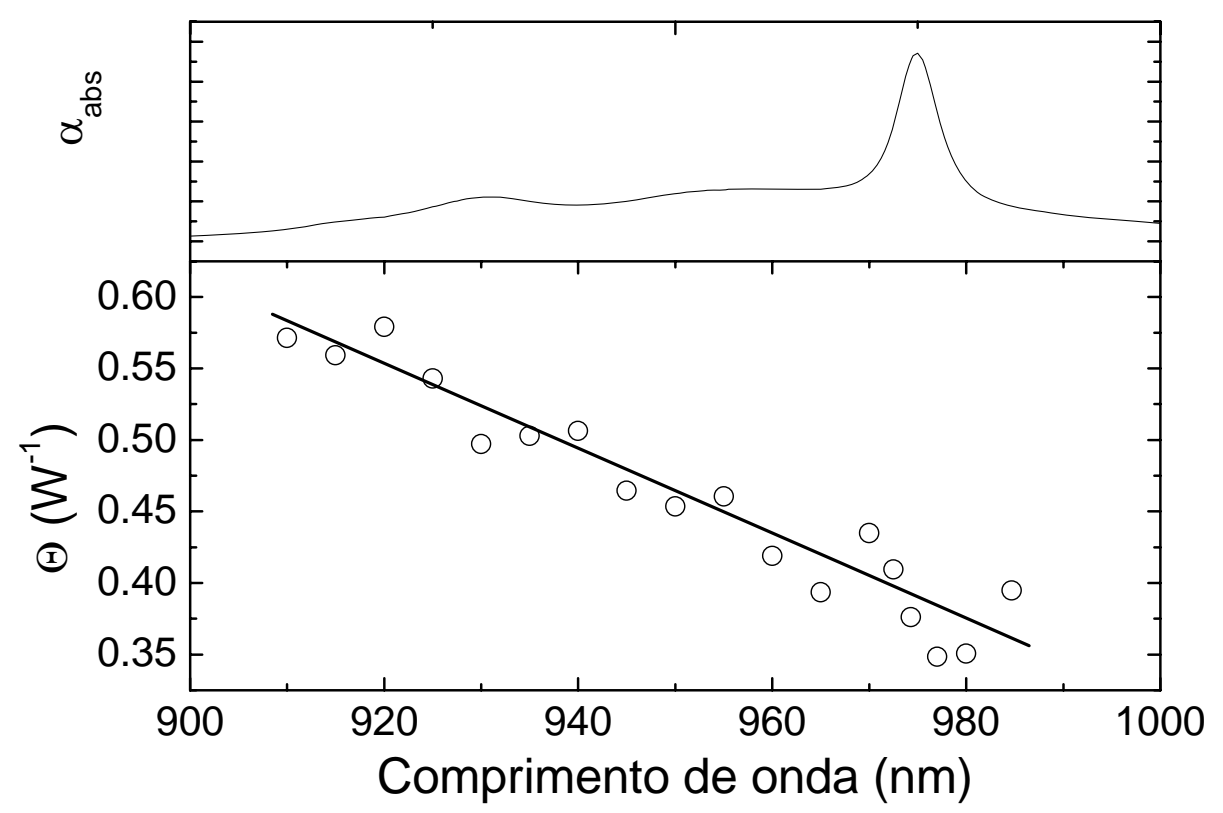

Figura 2-10 - Sinal da LT normalizado pela potência absorvida como função do comprimento de onda de bombeamento. Do ajuste linear foram obtidos $\eta=(0.92 \pm 0.08) \mathrm{e}$ $\mathrm{C}=(3.3 \pm 0.2) \mathrm{W}^{-1}$. Na parte superior temos o espectro de absorção, para comparação.

Desde que os parâmetros $\eta$ e $C$ são obtidos do ajuste linear da Figura 2-10, o método de LT com múltiplos comprimentos de onda não requer comparação com uma amostra de referência, o que normalmente aumentaria o erro na determinação destes parâmetros. Em geral, quanto maior o intervalo de comprimentos de onda acessados, maior a precisão do método. Entretanto o valor encontrado para h no vidro $\mathrm{QX} / \mathrm{Yb}$ tem um erro relativamente pequeno apesar do intervalo de comprimentos de onda acessado, o que mostra a precisão da técnica.

Como para materiais dopados com $\mathrm{Yb}^{3+}$ o decaimento multifônon pode ser desprezado devido à grande separação entre os dois níveis do íon, $\sim 10000 \mathrm{~cm}^{-1}$, espera-se que a eficiência quântica da 
fluorescência, $\eta$, seja de $100 \% .{ }^{4}$ No entanto, o valor encontrado indica que existe algum mecanismo de perda presente no material. O principal mecanismo que leva a redução em $\eta$ em sistemas dopados com $\mathrm{Yb}^{3+}$ é a transferência de energia para impurezas, que pode ser aumentada pela migração de energia devida a superposição entre as bandas de absorção e emissão do íon. Na Ref [1] pode ser vista uma redução na eficiência quântica de fluorescência de 0,93 para 0,59 para duas amostra de vidro fosfato dopadas com $\mathrm{Yb}^{3+}$ : a primeira livre de radicais de $\mathrm{OH}^{-}$e a segunda com os radicais. Esta redução indica que parte da energia de excitação original foi absorvida pelas impurezas, resultando num aumento da carga térmica, $\phi$, de 0,13 para 0,44 , respectivamente. Desta forma, como a amostra de QX apresenta $\eta$ similar ao da amostra sem $\mathrm{OH}^{-}$da Ref [1], podemos supor que ela também não apresenta este tipo de impureza, sendo a migração de energia sua principal fonte de perda.

Do valor da constate $C$ foi então calculado $d s / d T=(2,1 \pm 0,2) \times 10^{-6} \mathrm{~K}^{-1}$ para o comprimento de onda de prova de 632,8 nm. Este valor é próximo ao do vidro Q88, Tabela 2-1. Note que a constante $C \propto(\mathrm{ds} / \mathrm{dT}) / \mathrm{K}$, de modo que materiais com baixo valor de $\mathrm{C}$ são desejáveis pois apresentam efeitos térmicos menos pronunciados. $\mathrm{O}$ valor de $C$ para $\mathrm{o} \mathrm{QX} / \mathrm{Yb}$ está próximo àquele encontrado para outros vidros fosfatos manufaturados pela empresa, Q98, $C=(4,1 \pm 0,1) \mathrm{W}^{-1}$, e $\mathrm{Q} 100, C=(4,2 \pm 0,2) \mathrm{W}^{-1}$ [24]. Entretanto, este valor é pequeno quando comparado com vidros fluoretos e aluminatos; o ZBLAN, por exemplo, apresenta $C=(-12,5 \pm 0,8) \mathrm{W}^{-1}$ enquanto que o aluminato, LSCAS, apresenta $C=(13,2 \pm 0,8) \mathrm{W}^{-1}$. Além disso, é interessante notar que o valor de $\theta / P$ no pico de absorção indica o quanto da energia incidente é convertida em calor no íon. Para o vidro Q98 dopado com 1 massa\% de $\mathrm{Nd}^{3+}$ temos que $\theta / P=(0,54 \pm 0,02) \mathrm{W}^{-1}$ em $801,6 \mathrm{~nm}$, para o Q100 com 9 massa $\%$ de $\mathrm{Nd}^{3+} \theta / P=$ $(2,45 \pm 0,09) \mathrm{W}^{-1}$ para o mesmo comprimento de onda, enquanto que o $\mathrm{QX} / \mathrm{Yb}$ com 6,8 massa $\%$ de $\mathrm{Yb}^{3+}$ apresenta $\theta / P=(0,27 \pm 0,03) \mathrm{W}^{-1}$ em $975 \mathrm{~nm}$ [24]. Isto indica que apesar da alta dopagem o vidro

\footnotetext{
${ }^{4}$ Este valor é esperado porque como existem apenas 2 níveis neste intervalo, toda a população do nível superior deveria decair radiativamente para o nível inferior, ou seja, $\mathrm{W}_{\mathrm{R}} / \mathrm{W}_{\mathrm{T}}=1$.
} 
QX dopado com $\mathrm{Yb}^{3+}$ converte muito menos energia absorvida para calor que seus similares dopados com $\mathrm{Nd}^{3+}$. As propriedades térmicas tais como $D, K$, e $d s / d T$ para a maioria dos vidros fosfatos são similares, Tabela 2-1. Isto indica que estes parâmetros são governados pelos principais constituintes dos vidros. Comparados com outros vidros vemos que os fosfatos apresentam $D$ e $K$ menores que os vidros aluminatos (LSCAS) e similares aos fluoretos (ZBLAN) e calcogenetos.

\subsection{Espectroscopia de LT no vidro aluminato}

Foram realizadas também medidas de LT em um vidro aluminato com 6 massa $\%$ de $\mathrm{Yb}^{3+}$ e 2 $\mathrm{mm}$ de espessura. Através das medidas transientes no centro da linha ( 976 nm), Figura 2-11, foi obtido o tempo de formação da LT, $t_{c}$, à partir do qual foi calculado o valor da difusividade térmica do material, $D=(7,2 \pm 0,2) \times 10^{-3} \mathrm{~cm}^{2} / \mathrm{s}$ usando a relação $D=w_{o e}{ }^{2} / 4 t_{c}$. A condutividade térmica, $K$, pode então ser calculada através da relação $K=\rho c D$, onde $\rho=2.9 \mathrm{~g} / \mathrm{cm}^{3}$ é a densidade do material e $c=0.92 \mathrm{~J} / \mathrm{gK}$ seu calor específico. Desta forma encontramos $K=(2,0 \pm 0,6) \times 10^{-2} \mathrm{~W} / \mathrm{cmK}$.

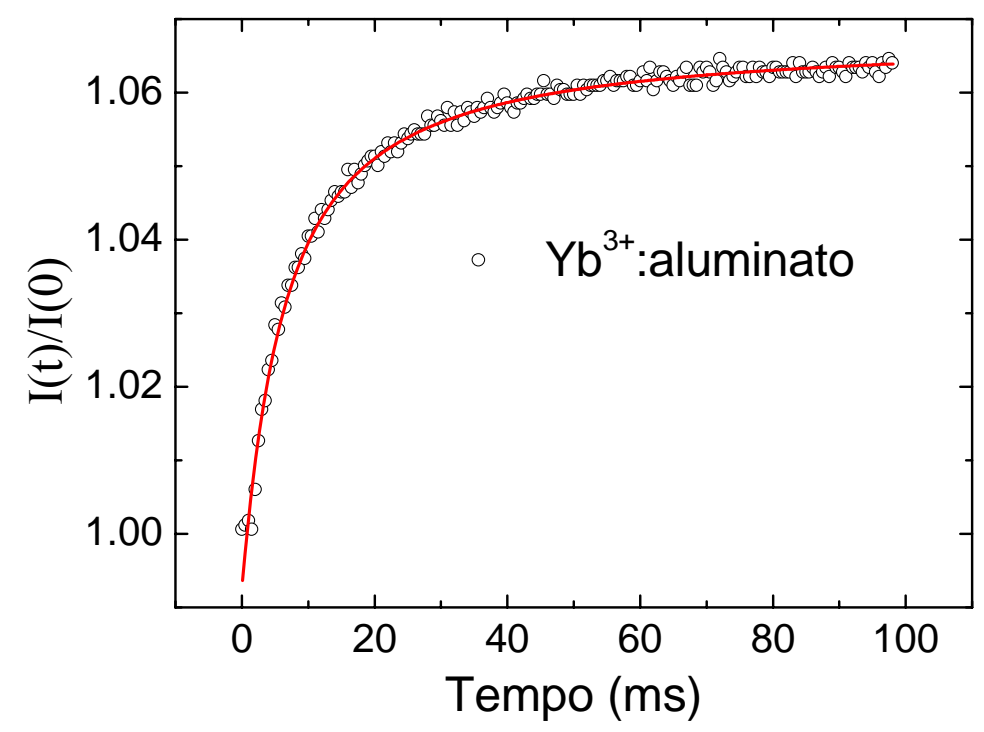

Figura 2-11 - Sinal transiente de LT para $\mathrm{P}=81 \mathrm{~mW}$ e $\lambda=976 \mathrm{~nm} \mathrm{em}$

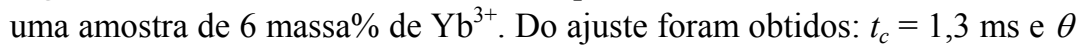
$=-0.07804$ rad. $\mathrm{L}=2 \mathrm{~mm}$. 
A técnica de LT em múltiplos comprimentos de onda também foi utilizada no vidro aluminato dopado com $\mathrm{Yb}^{3+}$ para a obtenção de $\eta$ e $C$. Os resultados obtidos foram semelhantes àqueles do QX/Yb. Na Figura 2-12 está exibido o espectro do sinal de LT normalizado pela potência absorvida. Do ajuste linear foram obtidos $C=(9,7 \pm 0,8) \mathrm{W}^{-1}$ e $\eta=(0,92 \pm 0,15)$. Usando então $K=(2,0 \pm$ $0,6) \times 10^{-2} \mathrm{~W} / \mathrm{cmK}$ calculamos $d s / d T=(12,1 \pm 1,3) \times 10^{-6} \mathrm{~cm}^{2} / \mathrm{s}$. Este valores estão em excelente acordo com aqueles obtidos para vidros aluminatos dopados com $\mathrm{Nd}^{3+}[25,26]$. Também aqui o erro estimado em cada ponto foi de aproximadamente $10 \%$.

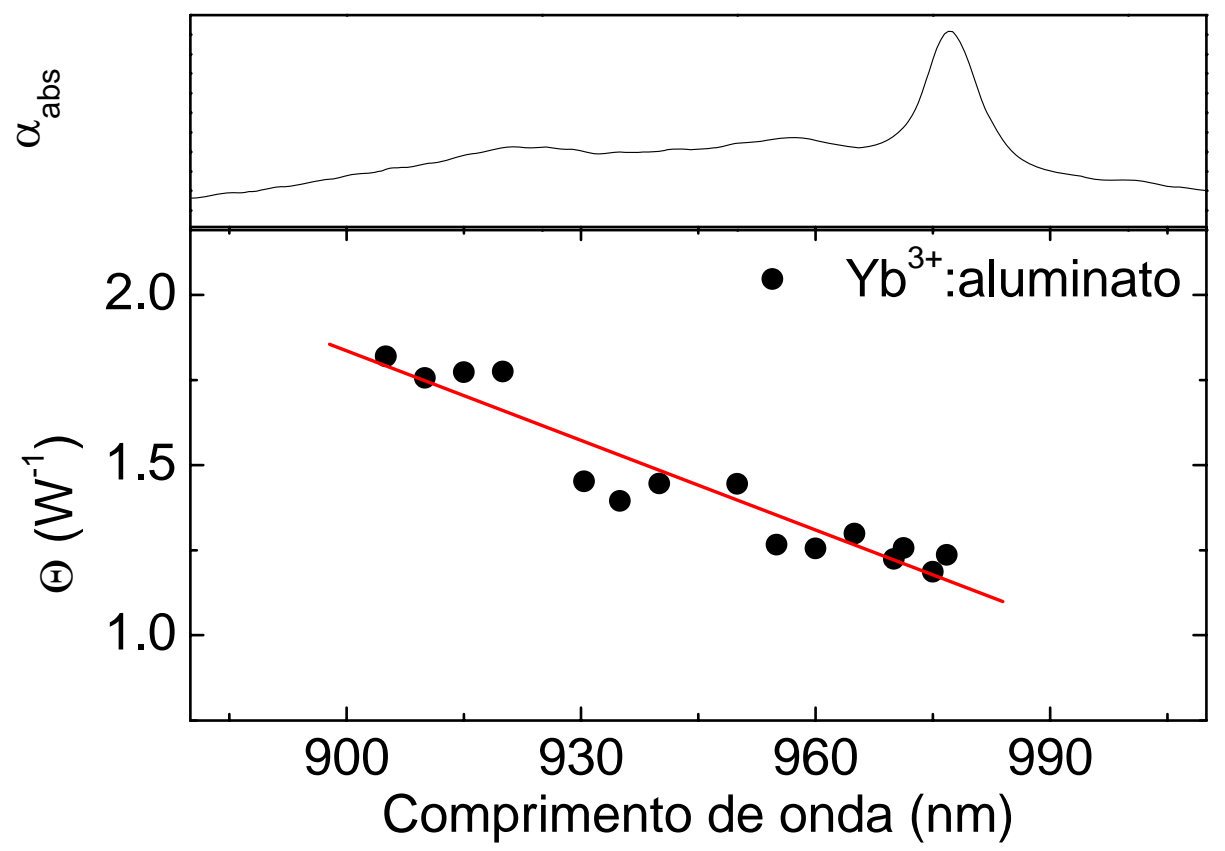

Figura 2-12 - Espectro do sinal de LT normalizado pela potência absorvida. Do ajuste linear foram obtidos $\mathrm{C}=(9,7 \pm 0,8) \mathrm{W}^{-1}$ e $\eta=(0,92 \pm 0,15)$.

Como no caso do $\mathrm{QX} / \mathrm{Yb}$ acreditamos que a principal fonte de perdas deste vidro seja devido à migração de energia visto que a eficiência quântica é alta, o que denota ausência de radicais de OH. Já o alto valor da constante $\mathrm{C}$ indica que os efeitos térmicos são mais pronunciados neste vidro que no fosfato. $\mathrm{O}$ valor de $d s / d T$ pôde também ser encontrado do ajuste do sinal de LT, no pico de absorção, normalizado por $\alpha_{a b s} L_{e f}$ como função da potência, Figura 2-13. 


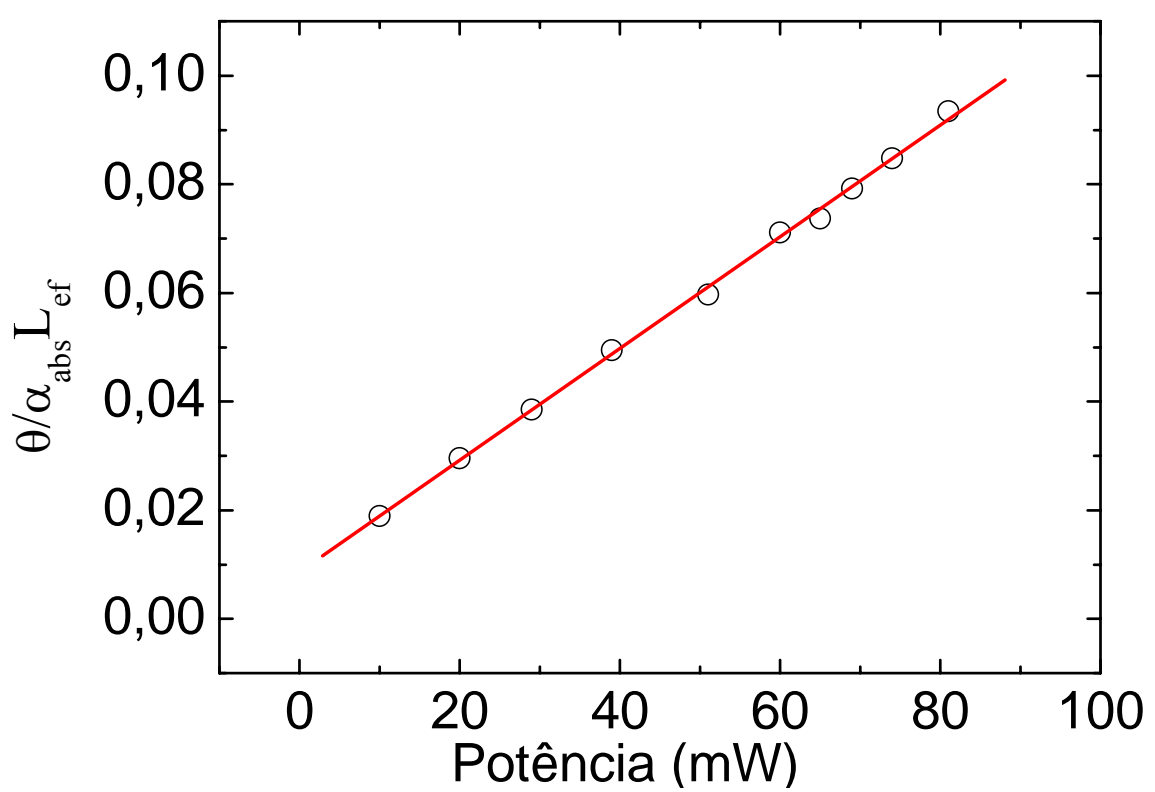

Figura 2-13 - Sinal de LT normalizado pela absorção e comprimento efetivo da amostra para o vidro aluminato. Os pontos experimentais foram ajustados com a (2-7.

Usando a eficiência quântica obtida da Figura 2-12, e comprimento médio de emissão sendo $1020 \mathrm{~nm}$, foi encontrado $d s / d T=(10,7 \pm 0,1) \times 10^{-6} \mathrm{~K}^{-1}$, que está em bom acordo, dentro da margem de erro, com o valor previamente obtido. Os valores encontrados para $D, K$ e $d s / d T$ concordam com aqueles encontrados por Andrade et al [25] e Astrath et al [26] para amostras dopadas com $\mathrm{Nd}^{3+}$. Isto indica claramente que as propriedades térmicas independem do íon dopante, pois eles estão em quantidade bem menor que os íons formadores da matriz.

Valores para os parâmetros fototérmicos de vários materiais laser estão listados na Tabela 2-1. A técnica de LT foi usada na obtenção destes parâmetros nos seguintes materiais: fluorozirconatos (ZBLAN), fluoroindatos (PGIZCa, ISZn, e InSBZnGdN), aluminosilicato (LSCAS), silicato, calcogeneto (GLS), calcohaleto $\left(\mathrm{PbI}_{2}-\mathrm{Sb}_{2} \mathrm{~S}_{3}-\mathrm{As}_{2} \mathrm{~S}_{3}\right)$, fluoroaluminato (YABC), e fosfatos (Q98, Q100). 
Tabela 2-1 - Parâmetros fototérmicos para diversos materiais laser, obtidos na literatura.

\begin{tabular}{|c|c|c|c|c|c|}
\hline Amostra & $\begin{array}{c}D \\
\left(10^{-3} \mathrm{~cm}^{2} / \mathrm{s}\right) \pm 5 \%\end{array}$ & $\begin{array}{c}K \\
\left(10^{-3} \mathrm{~W} / \mathrm{cmK}\right) \pm 10 \%\end{array}$ & $\begin{array}{c}d n / d T \\
\left(10^{-6} K^{-1}\right)\end{array}$ & $\begin{array}{c}d s / d T \\
\left(10^{-6} K^{-1}\right) \pm 10 \%\end{array}$ & Ref. \\
\hline Germanato $^{\text {d) }}$ & 2 & 4,5 & - & 1,4 & \\
\hline Aluminato ${ }^{d)}$ & 7,2 & 20 & - & 12,1 & \\
\hline $\mathrm{QX} / \mathrm{Yb}^{\mathrm{d})}$ & 4,5 & 10,1 & - & 2,1 & \\
\hline ISZn & 3,1 & 9,9 & - & $-1,3$ & {$[3]$} \\
\hline InSBZnGdN & 3,2 & 10,2 & - & $-2,1$ & {$[3]$} \\
\hline YABC & 3,3 & 8,2 & - & $-2,0$ & {$[3]$} \\
\hline ZBLAN & 2,6 & 7,7 & -12.0 & $-5,9$ & {$[3]$} \\
\hline LSCAS & 5,7 & 15,5 & 8,1 & 6,2 & {$[3]$} \\
\hline GaLaS & 2,7 & 4,3 & $26[27]$ & 2,6 & {$[3]$} \\
\hline $\mathrm{PbI}_{2}-\mathrm{Sb}_{2} \mathrm{~S}_{3}-\mathrm{As}_{2} \mathrm{~S}_{3}$ & 1,3 & - & - & - & {$[3]$} \\
\hline PGIZCa & 2,9 & 10,4 & - & $-2,4$ & {$[3]$} \\
\hline Soda lime ${ }^{\mathrm{c})}$ & 4,9 & 10 & - & - & {$[3]$} \\
\hline Sílica não dopada & 9,0 & 14,9 & 9,3 & 9,58 & [28] \\
\hline Q-98 (Kigre) & 2,2 & 5,5 & $-4,5$ & 1,31 & \multirow[t]{2}{*}{ [29] } \\
\hline Q-100 (Kigre) & 2,1 & 5,4 & $-4,6$ & 1,43 & \\
\hline LG-750 & 2,9 & 6,0 & $-5,1$ & $0,8^{\text {a) }}$ & [30] \\
\hline LG-760 & 4,3 & 8,4 & $-5,1$ & $0,6^{\text {a) }}$ & [31] \\
\hline LHG-8 & 2,7 & 5,8 & $-5,3$ & - & [30] \\
\hline Q-88 (Kigre) & 3,8 & 8,4 & $-0,5$ & $2,7^{\text {a) }}$ & [30] \\
\hline
\end{tabular}

Como mencionado acima, os valores obtidos para $d s / d T$ incluem $d n / d T$, o termo de expansão térmica e o de stress ótico. Para a maioria dos vidros e cristais óxidos, dependendo de sua composição/estrutura, os três termos na Equação 2-3 são positivos. Por outro lado, materiais como os fluoretos e fosfatos apresentam $d n / d T$ negativos, e são interessantes para minimizar $d s / d T$. Por exemplo, para Q-98 $d n / d T=-4.5 \times 10^{-6} \mathrm{~K}^{-1}$ assim $|d s / d T|<|d n / d T|$ devido ao sinal negativo deste. Da 
mesma forma vidros fluoretos apresentam $d s / d T$ negativo e também $|d s / d T|<|d n / d T|$ (para o ZBLAN $\left.d n / d T=-12 \times 10^{-6} \mathrm{~K}^{-1}\right)$. É possível perceber na tabela acima que em geral nos vidros óxidos ds $/ \mathrm{dT}>0$ enquanto que nos fluoretos ds $/ \mathrm{dT}<0$.

Assim, aplicamos a técnica de LT para obter o espectro térmico de materiais dopados com $\mathrm{Yb}^{3+} \mathrm{e}$ através deste espectro foram então calculados a constante $C$ e a eficiência quântica de fluorescência, $\eta$. Isto mostra a versatilidade desta técnica, que foi exaustivamente empregada em materiais dopados com $\mathrm{Nd}^{3+}$ e agora vem sendo aplicada a novos materiais.

\subsection{Conclusões do capítulo}

Neste capítulo vimos a importância do efeito térmico na variação do índice de refração. Foram introduzidos o conceito e a técnica de Lente Térmica. Esta técnica foi então aplicada a materiais dopados com $\mathrm{Yb}^{3+}$ para determinar importantes propriedades fototérmicas como a condutividade térmica, $K$, a difusividade térmica, $D$, o coeficiente de variação do caminho ótico com a temperatura, $d s / d T$ e a eficiência quântica de fluorescência, $\eta$. Para o vidro $\mathrm{QX} / \mathrm{Yb}$ foram obtidos os seguintes valores: $D=(4,5 \pm 0,4) \times 10^{-3} \mathrm{~cm}^{2} / \mathrm{s}, K=(10,1 \pm 0,9) \times 10^{-3} \mathrm{~W} / \mathrm{cmK}, C=(3,3 \pm 0,2) \mathrm{W}^{-1}, \eta=(0,92 \pm 0,08)$ e finalmente $d s / d T=(2,1 \pm 0,2) \times 10^{-6} / \mathrm{K}$. já para o vidro germanato, também dopado com $\mathrm{Yb}$ obtivemos $D=(2,0 \pm 0,1) \times 10^{-3} \mathrm{~cm}^{2} / \mathrm{s}, K=(4,5 \pm 0,3) \times 10^{-3} \mathrm{~W} / \mathrm{Kcm}, \quad \eta \sim 50 \%$ (estimado) e $d s / d T=1,4 \times 10^{-6} / \mathrm{K}$.

Por fim, para o vidro aluminato foi encontrado $D=(7,2 \pm 0,2) \times 10^{-3} \mathrm{~cm}^{2} / \mathrm{s}, \quad K=(2,0 \pm 0,6) \times 10^{-2}$ $\mathrm{W} / \mathrm{cmK}, C=(9,7 \pm 0,8) \mathrm{W}^{-1}$ e $\eta=(0,92 \pm 0,15)$ e $d s / d T=(12,1 \pm 1,3) \times 10^{-6} \mathrm{~cm}^{2} / \mathrm{s}$. Todas estas propriedades são importantes para prever o comportamento do feixe ao passar pelo meio, o que é importante no design de dispositivos óticos. 


\subsection{Referências}

[1] C. Jacinto, S. L. Oliveira, L. A. O. Nunes, T. Catunda, and M. J. V. Bell,Thermal lens study of the OH- influence on the fluorescence efficiency of Yb3+-doped phosphate glasses, Appl. Phys. Lett. 86 (2005) 071911.

[2] S. J. Sheldon, L. V. Knight, and J. M. Thorne,Laser-Induced Thermal Lens Effect - a New Theoretical-Model, App. Opt. 21 (1982) 1663-1669.

[3] S. M. Lima, J. A. Sampaio, T. Catunda, A. C. Bento, L. C. M. Miranda, and M. L. Baesso,Mode-mismatched thermal lens spectrometry for thermo-optical properties measurement in optical glasses: a review, J. Non-Crys. Sol. 273 (2000) 215-227.

[4] M. L. Baesso, J. Shen, and R. D. Snook,Mode-Mismatched Thermal Lens Determination of Temperature-Coefficient of Optical-Path Length in Soda Lime Glass at Different Wavelengths, J. App. Phys. 75 (1994) 3732-3737.

[5] W. Koechner, Solid-State Laser engineering, Springer - Verbag, New York, 1988.

[6] J. P. Gordon, R. C. C. Leite, R. S. Moore, S. P. S. Porto, and J. R. Whinnery,Long-Transient Effects in Lasers with Inserted Liquid Samples, J. App. Phys. 36 (1965) 3-6.

[7] C. Hu and J. R. Whinnery,New Thermooptical Measurement Method and a Comparison with Other Methods, App. Opt. 12 (1973) 72-79.

[8] C. Jacinto, A. A. Andrade, T. Catunda, S. M. Lima, and M. L. Baesso,Thermal lens spectroscopy of Nd : YAG, Appl. Phys. Lett. 86 (2005) 034104

[9] J. Shen, R. D. Lowe, and R. D. Snook,A Model for Cw Laser-Induced Mode-Mismatched DualBeam Thermal Lens Spectrometry, Chem. Phys. 165 (1992) 385-396.

[10] A. Taguchi, H. Nakagome, and K. Takahei,Thermal Quenching Mechanism of Yb Intra-4f-Shell Luminescence in Inp, J. App. Phys. 70 (1991) 5604-5607. 
[11] A. Taguchi, M. Taniguchi, and K. Takahei,Direct Verification of Energy Back Transfer from Yb 4f-Shell to Inp Host, Appl. Phys. Lett. 60 (1992) 965-967.

[12] S. G. Bishop, D. A. Turnbull, and B. G. Aitken,Excitation of emission in chalcogenide glasses by broadband Urbach edge absorption, J. Non-Crys. Sol. 266 (2000) 876-883.

[13] S. Q. Gu, D. A. Turnbull, and S. G. Bishop,Broad-band excitation of Pr3+ luminescence by localized gap state absorption in Pr:As12Ge33Se55 glass, IEEE Phot. Tech. Let. 8 (1996) 260262.

[14] D. A. Turnbull and S. G. Bishop,Rare earth dopants as probes of localized states in chalcogenide glasses, J. Non-Crys. Sol. 223 (1998) 105-113.

[15] S. M. Lima, A. S. S. de Camargo, L. A. O. Nunes, T. Catunda, and D. W. Hewak,Fluorescence quantum efficiency measurements of excitation and nonradiative deexcitation processes of rare earth 4f-states in chalcogenide glasses, Appl. Phys. Lett. 81 (2002) 589-591.

[16] J. A. Munoz, J. O. Tocho, and F. Cusso,Photoacoustic determination of the luminescent quantum efficiency of Yb3+ ions in lithium niobate, App. Opt. 37 (1998) 7096-7099.

[17] E. T. Y. Lee and E. R. M. Taylor,Compositional effects on the optical and thermal properties of sodium borophosphate glasses, J. Phys. Chem. Sol. 66 (2005) 47-51.

[18] www.kigre.com,

[19] G. Lei, J. E. Anderson, M. I. Buchwald, B. C. Edwards, R. I. Epstein, M. T. Murtagh, and G. H. Sigel,Spectroscopic evaluation of Yb3+-doped glasses for optical refrigeration, IEEE J. Quant. Elect. 34 (1998) 1839-1845.

[20] S. M. Lima, A. A. Andrade, R. Lebullenger, A. C. Hernandes, T. Catunda, and M. L. Baesso,Multiwavelength thermal lens determination of fluorescence quantum efficiency of solids: Application to Nd3+-doped fluoride glass, Appl. Phys. Lett. 78 (2001) 3220-3222. 
[21] C. Jacinto, S. M. Lima, and T. Catunda,Determination of fluorescence quantum efficiency in solutions by thermal lens measurements at several wavelengths: Application to Rhodamine 6G, J. Phys. IV 125 (2005) 225-227.

[22] A. A. Andrade, S. M. Lima, V. Pilla, J. A. Sampaio, T. Catunda, and M. L. Baesso,Fluorescence quantum efficiency measurements using the thermal lens technique, Rev. Sci. Inst. 74 (2003) 857-859.

[23] C. Jacinto, T. Catunda, D. Jaque, and J. G. Sole,Fluorescence quantum efficiency and Auger upconversion losses of the stoichiometric laser crystal NdAl[sub 3](BO[sub 3])[sub 4], Phys. Rev. B 72 (2005) 235111.

[24] S. M. Lima, D. N. Messias, L. A. O. Nunes, and T. Catunda,Nonlinear spectroscopy of rare earth doped materials, Anais de Óptica - XXVIII Encontro Nacional de Física da Matéria Condensada (2005) $243-246$.

[25] A. A. Andrade, T. Catunda, I. Bodnar, J. Mura, and M. L. Baesso,Thermal lens determination of the temperature coefficient of optical path length in optical materials, Rev. Sci. Inst. 74 (2003) 877-880.

[26] N. G. C. Astrath, J. H. Rohling, A. C. Bento, M. L. Baesso, C. Jacinto, S. M. Lima, L. A. O. Nunes, and T. Catunda,Fluorescence quantum efficiency in Nd2O3-doped aluminosilicate glasses by multiwavelength thermal lens method, J. Phys. IV 125 (2005) 185-187.

[27] S. M. Lima, T. Catunda, M. L. Baesso, L. D. Vila, Y. Messaddeq, E. B. Stucchi, and S. J. L. Ribeiro,Thermal-optical properties of Ga: La: S glasses measured by thermal lens technique, Journal of Non-Crystalline Solids 247 (1999) 222-226.

[28] N. G. C. Astrath, J. H. Rohling, A. N. Medina, A. C. Bento, M. L. Baesso, C. Jacinto, T. Catunda, S. M. Lima, F. G. Gandra, M. J. V. Bell, and V. Anjos,Time-resolved thermal lens 
measurements of the thermo-optical properties of glasses at low temperature down to $20 \mathrm{~K}$, Phys. Rev. B 71 (2005) 214202.

[29] C. Jacinto, S. L. Oliveira, L. A. O. Nunes, J. D. Myers, M. J. Myers, and T. Catunda,Normalized lifetimes thermal lens method for determination of luminescence quantum efficiency and thermo-optical coefficients: Application to $\mathrm{Nd}^{3+}$-doped glasses, (submitted)

[30] J. H. Campbell and T. I. Suratwala,Nd-doped phosphate glasses for high-energy/high-peakpower lasers, Journal of Non-Crystalline Solids 263 (2000) 318-341.

[31] S. A. Payne, C. D. Marshall, A. Bayramian, G. D. Wilke, and J. S. Hayden,Laser Properties of a New Average-Power Nd-Doped Phosphate-Glass, App. Phys. B 61 (1995) 257-266. 


\section{Capítulo 3}

\section{Efeitos populacionais sobre o índice de refração não linear}

Neste capítulo serão apresentados estudos sobre do índice de refração não-linear $\left(n_{2}\right)$ em sólidos dopados com íons de terras raras e metais de transição, que são materiais laser ativos. Este tema é muito importante e tem despertado a atenção de diversos pesquisadores devido às várias possíveis aplicações dos efeitos não-lineares e suas implicações no comportamento do laser.

\subsection{Dependência do índice de refração não linear com a população do estado excitado}

Em materiais dopados com íons terras-raras e metais de transição, a redistribuição de população entre os níveis de energia excitado e fundamental tem um papel fundamental na variação do índice de refração, $\Delta n$. Neste caso, esta redistribuição muda a susceptibilidade ótica total do sistema matriz + íon, $\chi$, e, desta forma, seu índice de refração.

A Figura 3-1 mostra um esquema de bombeamento típico para um sistema laser de estado sólido, onde o nível metaestável laser é excitado via relaxação não radiativa de um nível superior. Neste caso, o laser de bombeio não está em ressonância com a transição direta entre o estado fundamental e o metaestável, ou seja, não há efeitos ressonantes referentes ao nível laser superior. Esta figura mostra que sob o efeito do bombeio, uma parte da população permanece no estado fundamental enquanto outra parte vai para o nível excitado. 


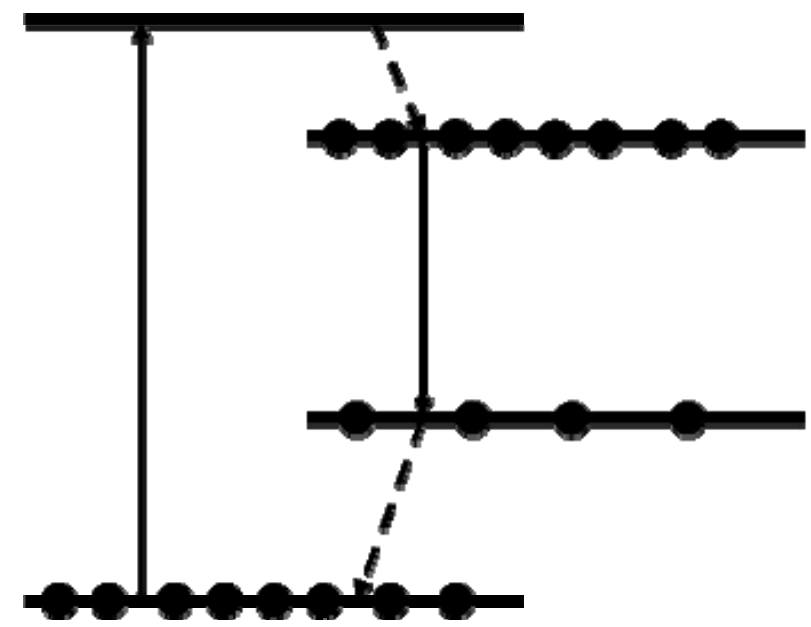

Figura 3-1 - Diagrama de níveis típico de um laser de estado sólido. As setas cheias indicam processos radiativos enquanto as tracejadas indicam processos não radiativos.

A dinâmica deste sistema pode ser descrita a partir de equações de taxa, supondo que o laser é ligado em $\mathrm{t}=0$, de forma que a população do estado excitado pode ser calculada através de:

$$
\frac{d N_{e x}}{d t}=\frac{\sigma I}{h v} N_{g}-\frac{N_{e x}}{\tau_{0}}
$$

com $N_{t}=N_{g}+N_{e x}$ a população total de íons no sistema, $N_{g}$ a população do estado fundamental, I é intensidade incidente e $v$ a freqüência do feixe incidente. Escrevendo a intensidade de saturação fora da ressonância como:

$$
I_{S}=\frac{h c}{\lambda \sigma \tau_{0}}
$$

onde $h$ é a constante de Planck, $\sigma$ é a seção de choque de absorção e $c$ é a velocidade da luz no vácuo. Pode-se resolver a Equação (3-1 de forma que o resultado final para a população do nível excitado é:

$$
N_{e x}(t)=N_{t} \frac{I / I_{s}}{1+I / I_{s}}\left(1-e^{-t / \tau}\right)
$$

Com isto, pode-se calcular a população do nível excitado a qualquer tempo e para qualquer valor da intensidade incidente. 
As primeiras observações de mudanças no índice de refração de sólidos dopados devido a efeitos populacionais foram feitas por Baldwin e Riedel em 1967 [1, 2]. Mais tarde, através de experimentos de mistura de onda [3] foi observado que esta redistribuição de população provocava uma mudança na susceptibilidade do meio da forma

$$
\chi=\chi_{m}+\frac{N_{g}}{N_{t}} \chi_{g}+\frac{N_{e x}}{N_{t}} \chi_{e x}
$$

onde $\chi_{\mathrm{m}}$ é a susceptibilidade da matriz, $\chi_{\mathrm{ex}}\left(\chi_{\mathrm{g}}\right)$ é a susceptibilidade da amostra quando todos os íons estão no estado excitado (fundamental). Entretanto, como $N_{t}=N_{g}+N_{e x}$ esta relação pode ser re-escrita como:

$$
\chi=\chi_{m}+\chi_{g}+\left(\chi_{e x}-\chi_{g}\right) \frac{N_{e x}}{N_{t}}
$$

O terceiro termo representa a contribuição da distribuição de população na susceptibilidade total do sistema. Note que apesar de depender das susceptibilidades de primeira ordem do estado excitado e do fundamental, este termo é proporcional ao quadrado do campo elétrico da onda incidente (devido ao fator $\mathrm{N}_{\mathrm{ex}}$ ), de forma que ele pode ser considerado como um termo efetivo de susceptibilidade de terceira ordem [4], ou

$$
\chi_{e f}^{(3)}=\left(\chi_{e x}^{(1)}-\chi_{g}^{(1)}\right) \frac{N_{e x}}{N_{t}}
$$

Para materiais laser dopados com íons emissores oticamente bombeados este termo pode produzir mudanças dependentes da intensidade na absorção e na dispersão do índice de refração complexo.

Por outro lado, a dependência do índice de refração com a intensidade pode ser obtida através da equação de Clausius-Mossoti [4](no sistema CGS): 


$$
\frac{3}{4 \pi} \frac{n^{2}-1}{n^{2}+2}=\chi_{m}+\chi_{g}+\frac{N_{e x}}{N_{t}}\left(\chi_{e x}-\chi_{g}\right)
$$

onde $n$ é o índice de refração do material, $\chi_{e x}\left(\chi_{\mathrm{g}}\right)$ é a susceptibilidade do estado excitado (fundamental) e $\chi_{m}$ é a susceptibilidade da matriz.

Supondo que $n=n_{0}+\Delta n \operatorname{com} \Delta n<<n_{0}$ e substituindo a Equação (3-3 na a Equação (3-7, podese desenvolver esta expressão para escrever o índice de refração linear, $n_{0}$, e a variação do índice de refração, $\Delta n$, como:

$$
\begin{aligned}
& n_{0}^{2}-1=4 \pi f_{L}^{2}\left(\chi_{m}+\chi_{g}\right) \\
& \mathrm{e} \\
& \Delta n=n_{2} I_{S} \frac{N_{e x}}{N_{t}}
\end{aligned}
$$

onde $f_{\mathrm{L}}=\left(n_{0}^{2}+2\right) / 3$ é o fator de Lorentz para a correção do campo local. Quando $I<<I_{S}$ e $t \rightarrow \infty$ então $\Delta n \approx n_{2} I$, que é a expressão usual para o caso de amostras excitadas em intensidades bem abaixo da saturação. $\mathrm{Na}$ (3-8 $n_{2}$ é o índice de refração não linear complexo dado por:

$$
n_{2}=\frac{2 \pi}{n_{0}} f_{L}^{2}\left(\frac{\chi_{e x}-\chi_{g}}{I_{S}}\right)
$$

Se $\chi_{i}$ for a susceptibilidade do nível $i$, então a polarizabilidade deste estado é dada por [5]:

$$
\alpha_{i}=\operatorname{Re}\left\{\chi_{i}\right\} / N_{t}
$$

Já a seção de choque de absorção deste nível é dada a partir da imaginária de $\chi_{i}$ através de:

$$
\sigma_{i}=\frac{8 \pi}{\lambda} \frac{f_{L}^{2}}{n_{0}} \frac{\operatorname{Im}\left\{\chi_{i}\right\}}{N_{t}}
$$


Usando estas relações podemos escrever as partes real e imaginária de $n_{2}$ como função da diferença de polarizabilidade, $\Delta \alpha=\alpha_{e x}-\alpha_{g}$, e de seção de choque de absorção, $\Delta \sigma=\sigma_{e x}-\sigma_{g}$, dos estados excitado e fundamental, respectivamente [5]:

$$
n_{2}^{\prime}=2 \pi \frac{f_{L}^{2}}{n_{0}} N_{t} \frac{\Delta \alpha}{I_{S}}
$$

$\mathrm{e}$

$$
n_{2}^{\prime \prime}=\frac{\lambda}{4 \pi} N_{t} \frac{\Delta \sigma}{I_{S}}
$$

Assim, a refração e a absorção não linear de um sistema típico dopado com íons emissores e bombeado oticamente dependem dos microparâmetros $\Delta \alpha$ e $\Delta \sigma$, que estão diretamente relacionados com as propriedades do íon ativo. Nesta análise a contribuição da matriz para o índice de refração não linear foi desprezada por ser algumas ordens de magnitude menor que a do íon dopante (em torno de 7 para alguns materiais) [6]. De modo que a não linearidade do íon é a dominante no sistema. Para o caso onde o feixe de excitação é gaussiano, a distribuição espacial da população (e daí, do índice de refração) também seguirá a forma gaussianaErro! Fonte de referência não encontrada.. Logo, o perfil de $\Delta n$ será semelhante ao de uma lente, semelhante ao caso térmico. Conseqüentemente este fenômeno é conhecido como Lente de População (LP). Como a população do estado excitado é, em primeira ordem, proporcional à intensidade incidente, $I$, o efeito de LP é equivalente ao efeito Kerr, onde $\Delta n=n_{2} I$. Assim, da mesma forma que para a LT, se $\mathrm{n}_{2}$ ' $>0$ a lente formada é convergente, caso contrário ela é divergente.

Analogamente à LT, o foco da LP pode ser calculado usando uma aproximação paraxial $(r<<w)$. Neste caso podemos escrever a variação de fase do campo elétrico ao atravessar uma LP como $\Delta \phi \cong k \operatorname{Ln}_{2}^{\prime} I_{0}\left(2 r^{2} / w^{2}\right), \operatorname{com} k=2 \pi / \lambda$. Por outro lado a fase introduzida por uma lente fina de distância 
focal $f$ sobre o campo elétrico quando este a atravessa é dada por $\Delta \phi=k r^{2} / 2 f$ [7]. Assim, o foco da lente de população pode ser escrito como:

$$
f_{L P}=\frac{\pi w^{4}}{8 L P n_{2}^{\prime}}
$$

com $\mathrm{P}$ a potência incidente sobre a LP.

Na seção seguinte introduziremos uma técnica para obter o valor de $n_{2}$. Com este valor será possível calcular o foco da LP e compará-lo com o da LT.

\subsubsection{A técnica de z-scan}

A caracterização das propriedades ópticas não lineares dos materiais é de grande interesse em vários campos da física, tanto do ponto de vista da física fundamental como da aplicada. Em particular um grande esforço vem sendo feito para a determinação da susceptibilidade óptica não linear de terceira ordem,$\chi^{(3)}$, responsável por fenômenos tais como geração de terceiro harmônico e autofocalização. Existem diversas técnicas usadas para a determinação de $\chi^{(3)}$, dentre elas podemos citar mistura degenerada de quatro ondas [8], mistura quase degenerada de três e quatro ondas [9], interferometria não linear [10], rotação de elipse [11], geração de terceiro harmônico [12], medidas de distorção de feixe [13] e experimentos fotoacústicos [14]. A técnica de varredura-Z (ou z-Scan como é mais conhecida) foi proposta em 1989 por Sheik-Bahae et al [15] como um método alternativo para a determinação de $\chi^{(3)}$. Esta técnica nos provê uma maneira sensível e direta de obter os valores e os sinais das partes real e imaginária da susceptibilidade óptica de terceira ordem, respectivamente, proporcional ao índice de refração não linear e ao coeficiente de absorção não linear. A simplicidade do aparato experimental e da análise dos dados contribuíram para que o método de $z$-scan tenha se tornado popular e largamente utilizado por diversos grupos de pesquisa. Esta técnica se baseia na idéia de relacionar a variação da intensidade no centro do feixe com a variação na fase (e consequentemente 
com a do índice de refração) induzida pelo bombeio. Isto é feito monitorando a transmitância, $T$, como função do caminho da amostra ao longo do feixe. A transmitância é aqui definida como a razão entre a intensidade perto do foco $I(\mathrm{z})$ e a intensidade longe do foco (que geralmente é pequena a ponto de não gerar efeitos não lineares).

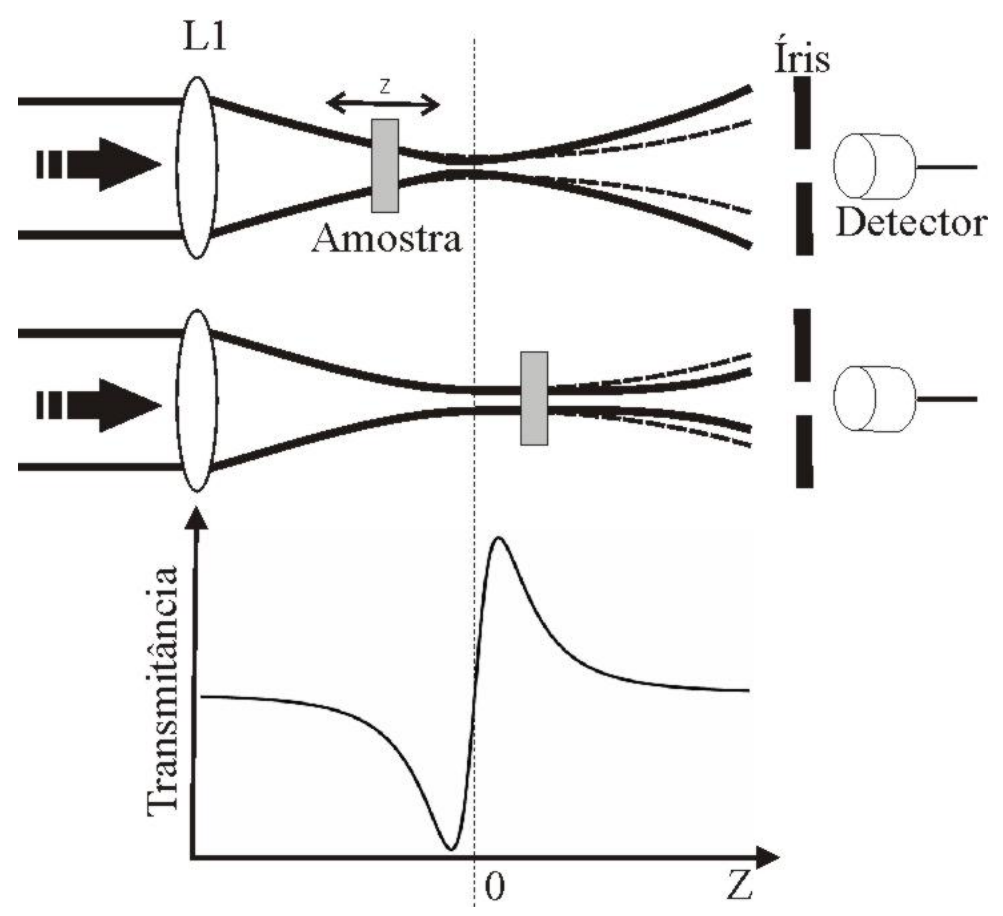

Figura 3-2 - Diagrama de construção da curva de z-scan. A parte superior da figura mostra o comportamento do feixe quando a amostra passa em torno do foco. A parte inferior da figura mostra a resposta da transmitância quando a amostra é deslocada. As linhas tracejadas indicam o caminho do feixe sem a amostra.

Na Figura 3-2 vemos uma curva típica de $z$-scan para um meio com $n_{2}{ }^{\prime}>0$ e para a curva refrativa (íris à frente do detector). Note que com a amostra em $z<0$ o foco da lente induzida está antes do foco de L1, de modo que o sinal chega mais espalhado no detector. Como menos luz está sendo captada na área do detector, a transmitância diminui até atingir o ponto de vale da curva. No caso da amostra em $z>0$ o foco induzido estará mais próximo do detector, de forma que mais luz estará entrando no mesmo. Este acréscimo na luz faz com que a transmitância aumente até atingir o pico da curva. A partir daí a intensidade do feixe diminui até não ser mais capaz de provocar variação na transmitância. Nesta figura temos que $z=0$ está no foco da lente L1. Para o caso de um meio com $n_{2}<$ 
0, o comportamento obtido é o inverso do mostrado acima, isto é, a posição de pico está em $z<0$ e a de vale em $z>0$.

Para o tratamento teórico da técnica de z-scan existem duas aproximações largamente utilizadas: o uso da Decomposição Gaussiana, DG, e a Integral de Difração de Fresnel-Kirchhoff, ID. Em ambos os casos o campo elétrico após a amostra, $E$ ', é escrito como o campo antes da amostra acrescido da mudança de fase induzida por ele mesmo:

$$
E^{\prime}=E e^{-i \Delta \phi}
$$

onde $\Delta \phi=\Delta \phi_{0} e^{-\frac{2 r^{2}}{w^{2}(z)}}$ e $\Delta \phi_{0}=(2 \pi / \lambda) L_{e f} I_{0} n_{2}$ 'é a máxima variação de fase induzida e $I_{0}=2 P / \lambda z_{c}$ é a intensidade no foco.

A diferença entre as duas técnicas está no tratamento dado à variação de fase $\Delta \phi$. No caso da DG esta variação de fase é desenvolvida em múltiplas Gaussianas da forma:

$$
E^{\prime}=E_{0}(z) \sum_{m=0}^{\infty} \frac{\left[i \Delta \phi_{0}\right]^{m}}{m !} \frac{w_{m 0}}{w_{m}} \exp \left(-\frac{r^{2}}{w_{m}^{2}}-\frac{i k r^{2}}{2 R_{m}}+i \theta_{m}\right)
$$

Definindo $d$ como a distância no espaço livre entre o meio e a abertura e $g=1+d / R(z)$, os outros parâmetros na Equação (3-16 são expressos como:

$$
\begin{gathered}
w_{m 0}^{2}=w^{2}(z) /(2 m+1) \\
d_{m}=\frac{1}{2} k w_{m 0}^{2} \\
R_{m}=d\left(1-\frac{g}{g^{2}+d^{2} / d_{m}^{2}}\right)^{-1}
\end{gathered}
$$




$$
\begin{gathered}
\theta_{m}=\tan ^{-1}\left(\frac{d / d_{m}}{g}\right) \\
w_{m}^{2}=w_{m 0}^{2}\left(g^{2}+d^{2} / d_{m}^{2}\right)
\end{gathered}
$$

com $w^{2}(\mathrm{z})$ e $R(\mathrm{z})$ dados pela teoria de propagação dos feixes Gaussianos [7]. Assim, o feixe inicial pode ser expresso como a soma de infinitos feixes Gaussianos de raios decrescentes. A transmitância referente à parte refrativa do sinal pode ser escrita como:

$$
T(z)=\frac{|E(z, \Delta \phi)|^{2}}{|E(z, \Delta \phi=0)|^{2}}
$$

Logo, usando a condição de campo distante $d>>z_{\mathrm{c}}, x=z / z_{c}$ e as Equações (3-16 até (3-21 a transmitância do feixe pode ser calculada no centro do feixe, $r=0$, como [15]:

$$
T(z)=1+\frac{4 x \Delta \Phi_{0}}{\left(1+x^{2}\right)\left(9+x^{2}\right)}
$$

No caso da ID, toma-se vantagem de que em geral o efeito não linear é muito pequeno comparado com o linear, de forma é possível se fazer a aproximação $e^{-i \Delta \phi} \sim 1-i \Delta \phi$. Assim, o campo elétrico passa a ser expresso como $E^{\prime}=E(1-\mathrm{i} \Delta \phi)$. Usando esta expressão na ID, Apêndice B, poderemos expressar o campo elétrico distante da amostra, $E_{a}$, em coordenadas cilíndricas, como:

$$
E_{a}=C \int_{0}^{\infty} e^{-\rho^{2}(1+i x)}(1-i \Delta \phi) J_{0}(\gamma \rho) \rho d \rho
$$

com $\mathrm{C}$ dado como no Apêndice B. Esta integral pode ser separada em duas partes, $E_{a}=C(\mu+\delta \mu)$ tal que: 


$$
\begin{aligned}
& \mu=\int_{0}^{\infty} e^{-\rho^{2}(1+i x)} J_{0}(\gamma \rho) \rho d \rho=\frac{\exp \left(-\frac{\gamma^{2}}{4(1+i x)}\right)}{2(1+i x)} \\
& \delta \mu=\int_{0}^{\infty} e^{-\rho^{2}(1+i x)} \Delta \phi(\rho) J_{0}(\gamma \rho) \rho d \rho
\end{aligned}
$$

Usando a variação de fase descrita na Equação (3-15 poderemos escrever $\delta \mu$ como:

$$
\begin{aligned}
\delta \mu & =-\frac{i \Delta \phi_{0}}{\left(1+x^{2}\right)} \int_{0}^{\infty} e^{-\rho^{2}(3+i x)} J_{0}(\gamma \rho) \rho d \rho \\
& =-\frac{i \Delta \phi_{0}}{\left(1+x^{2}\right)} \frac{\exp \left(-\frac{\gamma^{2}}{4(3+i x)}\right)}{2(3+i x)}
\end{aligned}
$$

Por outro lado, o perfil de intensidade no campo distante é dado por:

$$
I_{a}=\left|E_{a}\right|^{2} \propto|\mu+\delta \mu|^{2} \approx|\mu|^{2}+\mu \cdot \delta \mu^{*}+\delta \mu \cdot \mu^{*}
$$

Em que a aproximação $\delta \mu<<\mu$ é feita considerando-se que $\Delta \phi$ é pequeno. Finalmente, a transmitância normalizada pode ser calculada da mesma forma que na Equação (3-22, ficando $\mathrm{T}=|\mu+\delta \mu|^{2} /|\mu|^{2}$ ou equivalentemente.

$$
T(z)=1+2 \operatorname{Re}\left\{\frac{\delta \mu}{\mu}\right\}
$$

cujo resultado é igual à Equação (3-23.

Apesar de apresentarem resultados iguais para o caso descrito pela Equação (3-15, o método de DG não é facilmente aplicável a perfis de $\Delta \phi$ não Gaussianos [16]. Neste caso, a ID apresenta mais vantagens, pois mesmo sem oferecer uma resposta analítica, ainda é possível se obter um resultado numérico. Como exemplos de aplicação podemos citar os casos onde há saturação da não linearidade ou o efeito de upconversion Auger, como será visto nos capítulos seguintes. 
Da Equação (3-23 é possível então deduzir uma relação entre a variação da transmitância entre o pico e o vale da curva de transmitância, $\Delta T_{p v}$, com a fase induzida por:

$$
\Delta T_{p v} \sim 0.406\left(1-A_{b}\right)^{0.25} \Delta \phi_{0}
$$

$\operatorname{com} A_{b}=1-\exp \left(-r_{a}{ }^{2} / w^{2}\right)$ a transmitância da íris à frente do detector, $r_{a}$ e $w$ são os raios da abertura e do feixe, respectivamente. $\mathrm{O}$ fator $A_{b}$ foi introduzido pra levar em conta o efeito da abertura na medida [15]. Já a variação na distância de pico a vale está relacionada à distância confocal por:

$$
\Delta Z_{p v} \cong 1.73 z_{c}
$$

Caso a íris seja removida, estaremos coletando toda a luz no detector. Neste caso o sistema não será mais sensível à variação axial da intensidade e o sinal será constante; a menos que o meio possua algum tipo de absorção não linear. Neste caso teríamos $n_{2}{ }^{\prime \prime} \neq 0$, e a transmitância obtida num $z$-scan teria um perfil lorentziano e podendo ser ajustada por:

$$
T(z)=1+\frac{\Delta A}{1+x^{2}}
$$

$\operatorname{com} \Delta \mathrm{A}=(2 \pi / \lambda) \mathrm{L}_{\mathrm{ef}} \mathrm{I}_{0} \mathrm{n}_{2}$ " sendo a máxima variação induzida na transmitância.

\subsubsection{A técnica de Z-scan resolvida no tempo}

A normalização do sinal de z-scan (transmitância) da forma proposta por por Sheik-Bahae et al [15] é susceptível a erros decorrentes de maus polimentos ou falta de paralelismo das superfícies da amostra. Para contornar este problema em meios com não linearidade lenta $\left(\Delta n \sim N_{e x} \sim N_{t}\left(1-\mathrm{e}^{-t / \tau}\right) \operatorname{com} \tau\right.$ $\sim 100 \mu$ s ou maior) Oliveira et al[17] desenvolveram a técnica de $z$-scan resolvida no tempo, onde são 
aquisicionados os sinais transmitidos imediatamente após a abertura de um chopper, ${ }^{1}$ quando ainda não existem efeitos não lineares, e antes do seu fechamento, quando os efeitos não lineares estão presentes na amostra. A razão entre estes sinais define a transmitância normalizada. Na Figura 3-3 pode ser visto o efeito da não linearidade sobre o sinal incidente modulado, em diferentes posições da amostra ao longo do feixe.

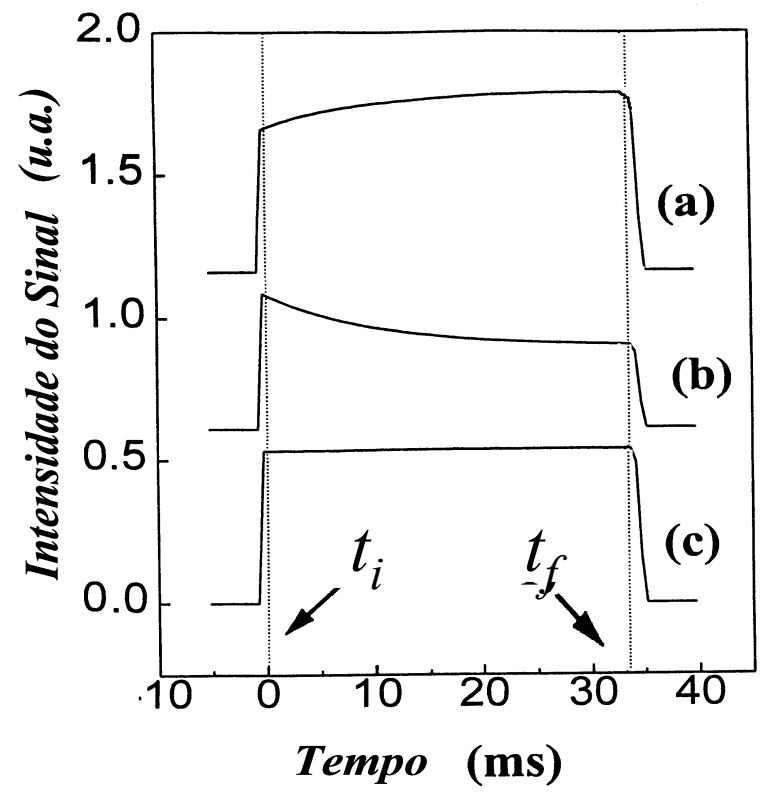

Figura 3-3 - Evolução temporal do sinal de z-scan da amostra de $\mathrm{GdAlO}_{3}: \mathrm{Cr}^{3+}\left(n_{2}{ }^{\prime}>0\right)$, colocada em (a) $z=0,85 z_{\mathrm{c}}$ (b) $z=-0,85 z_{\mathrm{c}}$ e (c) $z=7 z_{\mathrm{c}}$ [16].

Note que no caso (a) a amostra está logo após o foco onde a normalização do sinal detectado é maior que $1\left(I\left(\mathrm{t}_{\mathrm{f}}\right) / I(\mathrm{ti})>1\right)$, em (b) ela está antes do foco e $I\left(\mathrm{t}_{\mathrm{f}}\right) / I\left(\mathrm{t}_{\mathrm{i}}\right)<1$; enquanto que em (c) ela está longe do foco onde a população no estado excitado é desprezível e portanto não há variação do índice de refração não linear, logo $I\left(\mathrm{t}_{\mathrm{f}}\right) / I\left(\mathrm{t}_{\mathrm{i}}\right) \sim 1$. Note que a sensibilidade desta técnica permite resolver

\footnotetext{
${ }^{1}$ Um modulador que interrompe o feixe de excitação em intervalos regulares de tempo. No nosso caso, um disco perfurado girando com uma freqüência ajustável.
} 
distorções de fase da ordem de $\lambda / 940$ [17], o que representa um grande avanço em relação àquela da proposta inicial de Sheik-Bahae [15], que era de $\lambda / 250 .^{2}$

É importante notar que como o efeito absortivo está presente tanto no sinal da fenda fechada (abertura 50\%) como no da fenda aberta (abertura 100\%), para obter uma resposta unicamente devida à refração não linear devemos normalizar o sinal da fenda fechada pelo sinal da aberta, $i$. e., sinal $50 \% / 100 \%$.

\subsubsection{Arranjo experimental da técnica de z-scan resolvida no tempo}

As medidas de z-scan foram realizadas utilizando o aparato experimental mostrado na Figura 3-4. Duas lentes foram dispostas com os focos, $f \sim 10 \mathrm{~cm}$, coincidindo com a posição do chopper, diminuindo assim o tempo que a pá leva para interromper o feixe. A amostra é deslocada ao longo da direção de propagação do feixe laser, próximo à posição focal da última lente, $f \sim 10 \mathrm{~cm}$. Um divisor de feixe é então utilizado para separar o feixe transmitido em dois, de modo que se possa medir simultaneamente a parte real e a parte imaginária do índice de refração não-linear da amostra. A parte real devida à refração é medida pelo detector colocado atrás da íris (fenda), regulada para transmitir apenas a parte central do feixe. Já a parte imaginária, devida à absorção, é captada pelo outro detector. Os sinais lidos pelos detectores são então amplificados e analisados num microcomputador.

\footnotetext{
${ }^{2}$ Para calcularmos a distorção da frente de onda como função de $\lambda$ basta lembrar que para uma variação de fase de $2 \pi$ teremos avançado a frente de onda em $1 \lambda$. Como $\Delta \mathrm{T}_{\mathrm{pv}} \sim \Delta \phi$, sabendo o valor de $\Delta \mathrm{T}_{\mathrm{pv}}$ podemos usar uma regra de três simples e calcular o quanto isto significa em termos de $\lambda$.
} 


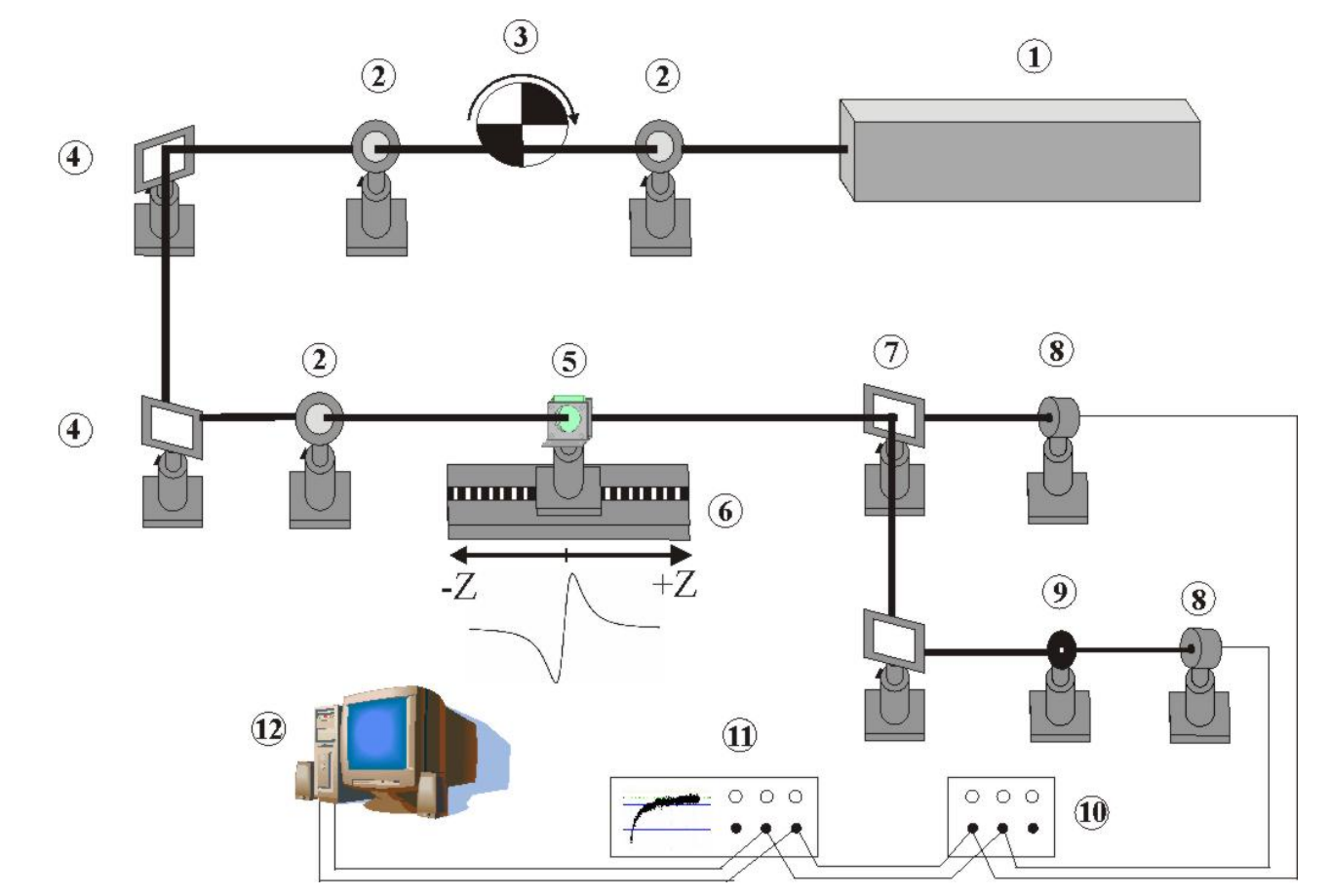

Figura 3-4 - Aparato experimental utilizado nas medidas de z-scan. 1 laser de excitação, 2 lentes, 3 chopper, 4 espelhos, 5 amostra, 6 estágio de translação, 7 divisor de feixes, 8 detectores, 9 íris, 10 amplificador, 11 osciloscópio e 12 computador de aquisição.

\subsubsection{Medidas de $\mathrm{z}$-scan em vidro fosfato dopado com $\mathrm{Yb}^{3+}$}

Serão agora apresentadas medidas de $z$-scan em uma amostra de vidro fosfato dopado com $8 \%$ de $\mathrm{Yb}^{3+}$ feita a vácuo no grupo do professor Luiz Antônio de Oliveira Nunes, para determinar seus parâmetros não lineares. Estas medidas foram realizadas com um laser de Ti:Safira sintonizado em 975 nm, que corresponde ao centro da linha de absorção.

A Figura 3-5 mostra a curva de $z$-scan da fenda aberta. Usando a Equação (3-31 obtivemos a parte imaginária do índice de refração não linear como sendo $n_{2}{ }^{\prime \prime}=1,71 \times 10^{-8} \mathrm{~cm}^{2} / \mathrm{W}$. Aplicando agora a Equação (3-13 calculamos a diferença de seção de choque de absorção como $\Delta \sigma=-2,3 \times 10^{-20}$ $\mathrm{cm}^{2}$. 


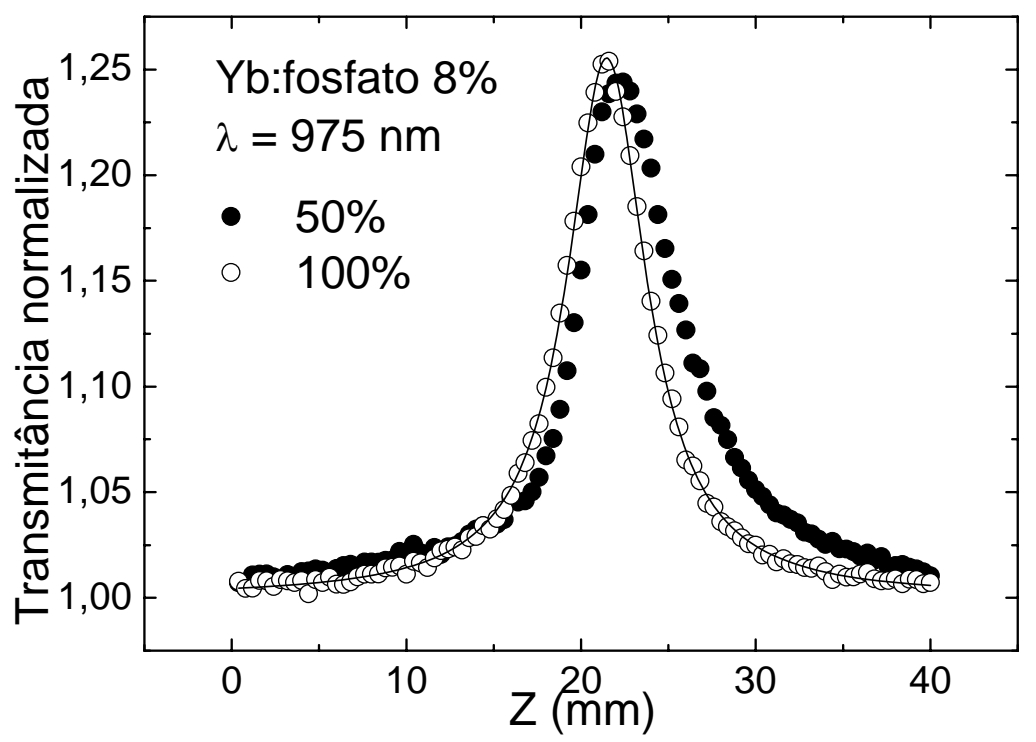

Figura 3-5 - Curva de z-scan absortivo (abertura 100\%) em uma amostra de $\mathrm{Yb}^{3+}$ :fosfato de $8 \%$. Dados do experimento: $P=100 \mathrm{~mW}, \alpha_{a b s}=7.8 \mathrm{~cm}^{-1}$, $L=1.77 \mathrm{~mm}, \omega_{0}=29 \mu \mathrm{m}$. Os círculos cheios foram obtidos para o detector com uma íris de $50 \%$ de transmitância.

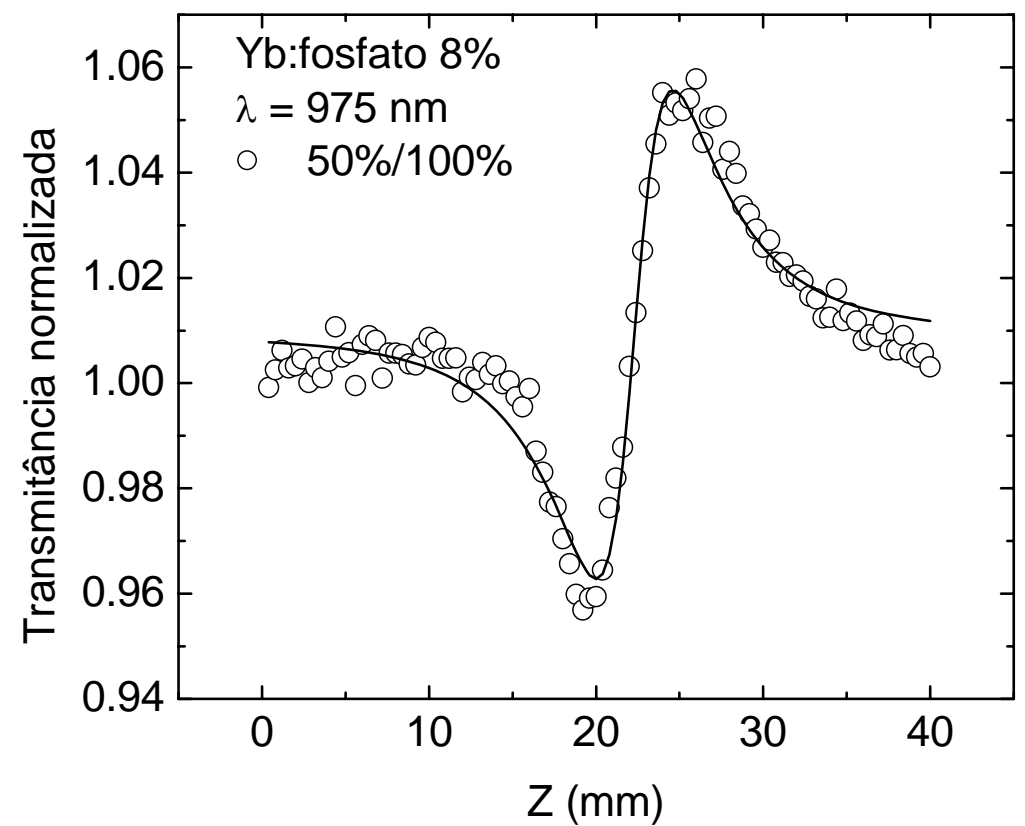

Figura 3-6 - Curva de $z$-scan, para a fenda fechada, já normalizada pela curva da fenda aberta. Aqui $P=100 \mathrm{~mW}$.

A Figura 3-6 mostra a curva de z-scan para a fenda fechada já normalizada pela curva da fenda aberta, ou seja, ele mostra a transmitância devida unicamente á não linearidade refrativa. Aplicando a Equação (3-29, que relaciona a variação entre o pico e o vale da curva da transmitância normalizada 
com a diferença de fase induzida na amostra, obtivemos a parte real do índice de refração não linear como $n_{2}{ }^{\prime}=1,77 \times 10^{-8} \mathrm{~cm}^{2} / \mathrm{W}$. Usando agora a Equação (3-12 podemos então calcular a diferença de polarizabilidade entre o estado excitado e fundamental como $\Delta \alpha=0,43 \times 10^{-25} \mathrm{~cm}^{3}$.

Dos resultados apresentados acima podemos ver que para o $\mathrm{Yb}^{3+}$ as contribuições absortiva e refrativa ao índice complexo de refração não linear são aproximadamente iguais. Este resultado é bem diferente

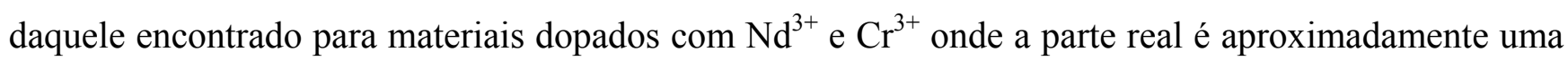
ordem de grandeza maior que a imaginária [16, 18-20]. A

Tabela 3-1 exibe valores de parâmetros não lineares de materiais dopados com $\mathrm{Nd}^{3+}$ e $\mathrm{Yb}^{3+}$ obtidos com a técnica de z-scan. ${ }^{3}$

Tabela 3-1 - Parâmetros não lineares de sólidos dopados com $\mathrm{Nd}^{3+} \mathrm{e} \mathrm{Yb}^{3+}$ obtidos com a técnica de $z$-scan. São mostrados: $\lambda(\mathrm{nm}), \tau(\mu \mathrm{s}), n_{2}^{\prime}\left(10^{-10} \mathrm{~cm}^{2} \mathrm{~W}^{-1}\right), n_{2}{ }^{\prime \prime}\left(10^{-12} \mathrm{~cm}^{2} \mathrm{~W}^{-1}\right), \Delta \sigma\left(10^{-21} \mathrm{~cm}^{2}\right)$ e $\Delta \alpha\left(10^{-26} \mathrm{~cm}^{3}\right)$.

\begin{tabular}{|c|c|c|c|c|c|c|}
\hline Amostra & $\lambda$ & $\tau$ & $n_{2}^{\prime}$ & $n_{2}^{\prime \prime}$ & $\Delta \sigma$ & $\Delta \alpha$ \\
\hline $\mathrm{Nd}^{3+}:$ InSBZnGdN $1 \%{ }^{\text {a) }}$ & 514 & 470 & $1,1,8$ & $-4,3$ & $-1,4$ & 2,7 \\
\hline $\mathrm{Nd}^{3+}:$ InSBZnGaN 2\% a) & 514 & 460 & 1,3 & 1,8 & 0,3 & 1,1 \\
\hline $\mathrm{Nd}^{3+}: \mathrm{YABC} 2 \%{ }^{\text {a) }}$ & 514 & 430 & 1,4 & 10,0 & 1,8 & 1,3 \\
\hline $\mathrm{Nd}^{3+}:$ PGIZCa $2 \%{ }^{\text {a) }}$ & 514 & 285 & 2,0 & 3,6 & 0,9 & 2,3 \\
\hline $\mathrm{Nd}^{3+}: Z B L A N 2 \%{ }^{a)}$ & 514 & 420 & 1,8 & 10,0 & 1,6 & 1,5 \\
\hline $\mathrm{Nd}^{3+}:$ YAG $0,44 \%{ }^{\text {b) }}$ & 514 & 250 & 1,4 & - & - & 8 \\
\hline $\mathrm{Nd}^{3+}:$ YAG $0,44 \%{ }^{\mathrm{b})}$ & 808 & 250 & 27 & $1,5 \times 10^{3}$ & -58 & 6,2 \\
\hline $\mathrm{Nd}^{3+}:$ YAG $0,75 \%{ }^{\text {b) }}$ & 514 & 230 & 1,8 & - & - & 6,5 \\
\hline $\mathrm{Nd}^{3+}:$ YAG $0,75 \%$ b) & 808 & 230 & 56 & $2,2 \times 10^{3}$ & -51 & 6,2 \\
\hline $\mathrm{Yb}^{3+}:$ Fosfato $8 \%{ }^{\mathrm{c}}$ & 976 & 1250 & $1,77 \times 10^{2}$ & $1,71 \times 10^{4}$ & -23 & 4,3 \\
\hline
\end{tabular}

\footnotetext{
${ }^{3}$ Diversas outras medidas de $z$-scan foram realizadas em diferentes materiais e serão apresentadas nos capítulos seguintes.
} 
Desta tabela podemos notar que em materiais dopados com $\mathrm{Nd}^{3+}$ a parte imaginária de $n_{2}$ é muito menor do que a real, em $514 \mathrm{~nm}$. Assim, estes materiais apresentam uma variação da refração não linear muito maior que da absorção não linear, o que pode ser apropriado para dispositivos chaveamento ótico. Entretanto, para $\lambda=808 \mathrm{~nm}$, estes valores tem a mesma ordem de grandeza, embora a parte real ainda seja maior que a imaginária. Para o vidro fosfato dopado com $\mathrm{Yb}^{3+}$, estudado nesta seção, os valores de $n_{2}$ ' e $n_{2}$ " são praticamente iguais e são ao menos uma ordem de grandeza maior que no caso do $\mathrm{Nd}^{3+}$. Esta característica pode ser importante para chaveamento ótico, mas pode ser depreciativa no caso de sistemas laser de alta potência devido às deformações que ela provoca na frente de onda.

\subsection{Distâncias focais induzidas devido aos efeitos térmico e eletrônico ${ }^{4}$}

Usando os dados da Tabela 2-1 e da

Tabela 3-1, e aplicando a Equação (3-14 e a Equação 2-4 podemos calcular as distâncias focais induzidas pelos efeitos de LT e LP para diversos materiais dopados com $\mathrm{Nd}^{3+}$ e bombeados em 514 $\mathrm{nm}$, Tabela 3-2. Para isto supomos que $w=30 \mu \mathrm{m}, P=100 \mathrm{~mW}, \phi=0,51$ para $\lambda=514 \mathrm{~nm}, \phi=0,23$ para $\lambda=808 \mathrm{~nm} \mathrm{e} \phi=0,12$ para $\lambda=976 \mathrm{~nm} .^{5} \mathrm{Na}$ mesma tabela está mostrada a razão entre os focos térmico e eletrônico. Esta razão nos permite estimar qual das contribuições é a mais importante na variação do índice de refração não linear para cada material estudado.

\footnotetext{
${ }^{4} \mathrm{O}$ efeito ao qual estamos nos referindo é aquele devido apenas à redistribuição de população nos íons emissores.

${ }^{5} \mathrm{O}$ termo $\phi$ deve ser incluído no denominador da Equação 2-4 para levar em conta que em sólidos dopados com íons emissores nem toda a luz absorvida é convertida em calor.
} 
Tabela 3-2 - Distâncias focais devidas às lentes térmica e de população, em materiais dopados com $\mathrm{Nd}^{3+} \mathrm{e} \mathrm{Yb}^{3+}$.

\begin{tabular}{|c|c|c|c|c|}
\hline Amostra & $\lambda(\mathrm{nm})$ & $f_{\mathrm{LT}}(\mathrm{cm})$ & $f_{\mathrm{LP}}(\mathrm{cm})$ & $f_{L P} / f_{L T}$ \\
\hline $\mathrm{Nd}^{3+}:$ InSBZnGaN $2 \%{ }^{\mathrm{a})}$ & 514 & $-2,7$ & 24,46 & -9 \\
\hline $\mathrm{Nd}^{3+}: \mathrm{YABC} 2 \%{ }^{\mathrm{a})}$ & 514 & $-2,3$ & 22,72 & -10 \\
\hline $\mathrm{Nd}^{3+}:$ PGIZCa $2 \%{ }^{a)}$ & 514 & $-2,4$ & 15,90 & $-6,6$ \\
\hline $\mathrm{Nd}^{3+}: Z B L A N 2 \%{ }^{a)}$ & 514 & $-0,7$ & 17,67 & $-24,4$ \\
\hline $\mathrm{Nd}^{3+}:$ YAG $0,44 \%{ }^{\mathrm{b})}$ & 514 & 5,1 & 22,7 & 4,4 \\
\hline $\mathrm{Nd}^{3+}:$ YAG $0,44 \%{ }^{\mathrm{b})}$ & 808 & 11,4 & 1,2 & 0,1 \\
\hline $\mathrm{Nd}^{3+}:$ YAG $0,75 \%{ }^{\text {b) }}$ & 514 & 5,1 & 17,7 & 3,4 \\
\hline $\mathrm{Nd}^{3+}:$ YAG $0,75 \%$ b) & 808 & 11,4 & 0,56 & 0,05 \\
\hline $\mathrm{Yb}^{3+}:$ Fosfato $8 \%{ }^{\mathrm{c})}$ & 976 & 8,954 & 0,18 & 0,02 \\
\hline
\end{tabular}

${ }^{\text {a) }}[18] ;{ }^{\text {b) }}[21] ;{ }^{\text {c) }}$ este trabalho.

Da Tabela 3-2 pode-se perceber que para materiais dopados com $\mathrm{Nd}^{3+}$ e bombeados em $514 \mathrm{~nm}$ a lente térmica é muito mais pronunciada que a de população, $\left|f_{\mathrm{LP}} / f_{\mathrm{LT}}\right|>1$, indicando que a LT é efeito o dominante na variação do índice de refração. Quando o bombeamento é feito em $808 \mathrm{~nm}$ a LP torna-se mais importante, $\left|f_{\mathrm{LP}} / f_{\mathrm{LT}}\right|<1$. Isto acontece porque o calor gerado em $808 \mathrm{~nm}$ é aproximadamente a metade daquele gerado em $514 \mathrm{~nm}$. Além disso, a LP é uma ordem de grandeza maior em $808 \mathrm{~nm}$ do que em $514 \mathrm{~nm}$, pois neste comprimento de onda a absorção é 16 vezes maior que em $514 \mathrm{~nm}$, com isso a intensidade de saturação diminui, o que aumenta $n_{2}{ }^{\prime}\left(n_{2}{ }^{\prime} \propto \Delta \alpha / I_{S}\right.$ e considerando $\Delta \alpha$ aproximadamente constante para estes comprimentos de onda). Para o fosfato dopado com $\mathrm{Yb}^{3+}$ também vemos que a LP é muito maior que a LT. As principais causas deste comportamento são, de novo, a pequena carga térmica dos íons de $\mathrm{Yb}^{3+}$ e o grande valor de $n_{2}$ ' (duas ordens de grandeza maior 
que para os materiais dopados com $\mathrm{Nd}^{3+}$ bombeados em $514 \mathrm{~nm}$ e uma ordem de grandeza maior que estes materiais bombeados em $808 \mathrm{~nm}$ ).

\subsection{Conclusões do capítulo}

Neste capítulo foi discutida a origem do efeito não linear em sólidos dopados e a importância do efeito eletrônico na variação do índice de refração. Foi introduzido o conceito lente de população, assim como o método utilizado para quantificar este efeito: a técnica de $z$-scan. Esta técnica permite a obtenção das quantidades microscópicas $\Delta \alpha$ e $\Delta \sigma$ a partir da determinação experimental de $n_{2}$. Utilizamos o foco não linear gerado pelos efeitos térmico e eletrônico para estimar a relação de grandeza entre estes efeitos.

No próximo capítulo abordaremos o efeito da saturação da não linearidade eletrônica sobre a técnica de $z$-scan e o modo como esta saturação pode influenciar os resultados obtidos. 


\subsection{Referências}

[1] E. P. Riedel and G. D. Baldwin, Theory of Dynamic Optical Distortion in Isotropic Laser Materials, J. App. Phys. 38 (1967) 2720-2725.

[2] G. D. Baldwin and E. P. Riedel, Measurements of Dynamic Optical Distortion in Nd-Doped Glass Laser Rods, J. App. Phys. 38 (1967) 2726-2738.

[3] R. C. Powell, S. A. Payne, L. L. Chase, and G. D. Wilke, 4-Wave Mixing of Nd-3+-Doped Crystals and Glasses, Phys. Rev. B 41 (1990) 8593-8602.

[4] R. C. Powell, Physics of Solid-State Lasers Materials, Springer-Verlag, New York, 1998.

[5] R. W. Boyd, Nonlinear Optics, Academic Press, San Diego, 1992.

[6] R. L. Sutherland, Handbook of Nonlinear Optics, Marcel Dekker, New York, 1996.

[7] A. Yariv, Optical Electronics, Saunders College Publishing, New York, 1991.

[8] S. R. Friberg and P. W. Smith, Nonlinear Optical-Glasses for Ultrafast Optical Switches, IEEE J. Quant. Elect. 23 (1987) 2089-2094.

[9] R. Adair, L. L. Chase, and S. A. Payne, Nonlinear Refractive-Index Measurements of Glasses Using 3-Wave Frequency Mixing, J. Opt. Soc. Am. B 4 (1987) 875-881.

[10] M. J. Moran, C. Y. She, and R. L. Carman, Interferometric Measurements of Nonlinear Refractive-Index Coefficient Relative to Cs2 in Laser-System-Related Materials, IEEE J. Quant. Elect. Qe11 (1975) 259-263.

[11] A. Owyoung, Ellipse Rotation Studies in Laser Host Materials, IEEE J. Quant. Elect. Qe 9 (1973) 1064-1069.

[12] P. D. Maker and R. W. Terhune, Study of Optical Effects Due to an Induced Polarization Third Order in Electric Field Strength, Phys. Rev. 137 (1965) A801-A818.

[13] W. E. Williams, M. J. Soileau, and E. W. Vanstryland, Optical Switching and N2 Measurements in Cs2, Opt. Comm. 50 (1984) 256-260. 
[14] Y. Bae, J. J. Song, and Y. B. Kim, Photo-Acoustic Study of 2-Photon Absorption in Hexagonal Zns, J. App. Phys. 53 (1982) 615-619.

[15] M. Sheik-bahae, A. A. Said, and E. W. V. Stryland, High sensitivity, single-beam $n_{2}$ measurements, Opt. Lett. 14 (1989) 955.

[16] L. C. Oliveira, T. Catunda, and S. C. Zilio, Saturation effects in Z-scan measurements, Jap. J. App. Phys. 35 (1996) 2649-2652.

[17] L. C. Oliveira and S. C. Zilio, in Appl. Phys. Lett., Vol. 65, 1994, p. 2121-2123.

[18] A. A. Andrade, T. Catunda, R. Lebullenger, A. C. Hernandes, and M. L. Baesso, Electronic and thermal contributions to the non-linear refractive index of $N d 3+$ ion-doped fluoride glasses, J. Non-Crys. Sol. 273 (2000) 257-265.

[19] S. M. Lima, H. Jiao, L. A. O. Nunes, and T. Catunda, Nonlinear refraction spectroscopy in resonance with laser lines in solids, Opt. Lett. 27 (2002) 845-847.

[20] T. Catunda and L. A. Cury, Transverse Self-Phase Modulation in Ruby and Gdalo3-Cr-+3 Crystals, J. Opt. Soc. Am. B 7 (1990) 1445-1455.

[21] A. A. C. Andrade, Tese de Doutorado, Instituto de Física de São Carlos -USP, São Carlos, 2002. 


\section{Capítulo 4}

\section{Efeitos de saturação sobre as medidas de z-scan}

\section{1. $\quad$ Efeitos de Saturação sobre as medidas de z-scan}

Como dito anteriormente no Capítulo 3, em materiais dopados com íons emissores, a não linearidade se origina da diferença de população entre o nível metaestável excitado e o nível fundamental, que têm susceptibilidades complexas diferentes entre si. Assim a susceptibilidade do meio muda proporcionalmente à população do estado excitado metaestável bombeado pelo laser. Diversos experimentos foram realizados na tentativa de se caracterizar a não linearidade em sólidos dopados, devido aos efeitos relacionados a ela tais como spatial hole burning, auto focalização e modulação temporal de fase [1]. Inicialmente experimentos de mistura degenerada de quatro ondas, e de grades de população foram empregados para determinar a não linearidade [2-4]. No entanto, estes experimentos não permitiam a discriminação entre os efeitos absortivos e refrativos, pois o sinal obtido era proporcional ao módulo quadrado da susceptibilidade não linear. A primeira medida direta da parte real de $n_{2}$ foi realizada em cristais dopados com $\mathrm{Cr}^{3+}$, rubi e $\mathrm{GdAlO}_{3}$, utilizando uma técnica interferométrica [5]. Além desta técnica, a mistura não degenerada de duas ondas foi posteriormente utilizada para distinguir entre $n_{2}$ ' e $n_{2}$ ” $[6,7]$, produzindo uma boa concordância com os resultados da Ref [5]. Contudo, a introdução da técnica de z-scan em 1989 [8], permitiu uma determinação mais fácil e precisa das partes real e imaginária de $n_{2}$, fazendo com que a técnica se tornasse bastante conhecida e empregada nos laboratórios de ótica em todo o mundo. No entanto, até agora uma grande parte das pesquisas feitas com a técnica de z-scan foi realizada sob condições onde não há o efeito de 
saturação da não linearidade. ${ }^{1}$ Contudo, é bem conhecido que uma grande classe de materiais tem não linearidades que saturam com a intensidade da luz, e.g., vidros dopados com microcristais semicondutores [9], cristais dopados com íons emissores [10-12], vapor atômico [13], materiais orgânicos [14], etc. Assim se fez necessário introduzir mudanças na técnica de z-scan de forma a levar em consideração este efeito de saturação. Em outras técnicas, como as de mistura de onda, foram também documentados os efeitos da saturação. Neste caso, um desvio do comportamento esperado surgiu quando as intensidades utilizadas se aproximavam da intensidade de saturação. Neste regime de intensidade foi observado um aumento na divergência do feixe transmitido, que foi atribuído ao efeito de saturação. Algumas tentativas foram feitas de se considerar o efeito da saturação em experimentos de mistura de onda [12] e modulação transversal de fase [11]. Para o z-scan, alguns trabalhos também já foram publicados tratando deste tema, mas em geral eles estão sujeitos a condições restringentes como necessitar de resolução numérica da integral de difração [15]; ou as aproximações são válidas apenas até $I \sim I_{S}$ [16]. Mais recentemente uma expressão analítica pôde ser obtida para um sistema de dois níveis [17], o que limita bastante sua aplicabilidade.

Para os meios com não linearidade ótica saturável a variação do índice de refração devido à intensidade incidente se escreve como:

$$
\Delta n(r)=\frac{n_{2}^{\prime} I(r)}{1+I(r) / I_{S}}
$$

O efeito desta saturação sobre $\Delta n$ é fazer com que o perfil de índice de refração induzido na amostra pelo laser deixe de ser gaussiano e passe a apresentar um perfil achatado no centro, Figura 4.1 [11]. Nesta figura $S=I / I_{S}$ é chamado parâmetro de saturação. O efeito de achatamento faz com que a lente induzida focalize numa posição diferente daquela relativa à lente não saturada. Desta forma, uma

\footnotetext{
${ }^{1}$ No presente trabalho o termo saturação se aplica a meios onde $n_{2}$ deixa de responder proporcionalmente à potência incidente, a partir de um determinado valor.
} 
de medida de Z-scan poderia, se não considerar este efeito, levar a um resultado errado. Por isso, para o estudo das propriedades óticas, especialmente de sólidos dopados, é necessário se levar este fato em consideração.

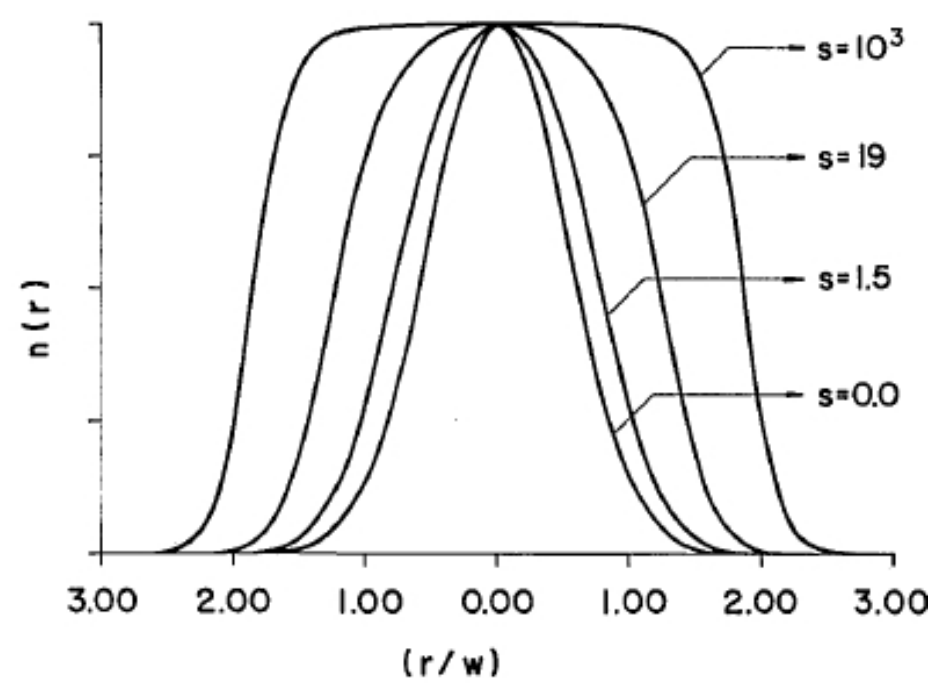

Figura 4.1 - Perfil radial do índice de refração, n(r) para diferentes parâmetros de saturação, $S=I / I_{S}$. O efeito da saturação no centro do feixe torna o perfil de n(r) achatado [11].

Oliveira et al[18] conseguiram à partir de simulações numéricas obter expressões empíricas aproximadas para a variação da transmitância da fenda fechada e da aberta (devido a refração e absorção não linear, respectivamente) corrigindo assim os efeitos da saturação. O resultado encontrado para $\Delta T_{p v}$ neste caso foi:

$$
\Delta T_{p v}=0,406\left(1-A_{b}\right)^{0,25(1+S)^{\left(1-A_{b}\right)}} \frac{\Delta \phi_{0}}{(1+S)^{0,525}}
$$

onde $\Delta \phi_{0}=(2 \pi / \lambda) L_{e f} n_{2} I_{0}$ é dado como no caso não saturado, I $I_{0}$ é a intensidade do feixe no foco da lente e $A_{b}$ é a transmitância da íris. Para o caso da íris totalmente aberta foi obtida a expressão

$$
T_{p}-1=\frac{2 \pi}{\lambda} n_{2}^{\prime \prime} \frac{I}{(1+S)^{0,76}}
$$


onde $T_{p}$ é a transmitância de pico na varredura z para o caso da íris aberta. Com estas duas expressões é possível então se obter os valores das partes real e imaginária do índice de refração não linear, $n_{2}$, e, por conseguinte calcular os valores dos microparâmetros $\Delta \alpha$ e $\Delta \sigma$. Note que para meios onde $I_{S}>>I_{0}$ retornamos ao caso de meios sem saturação na não linearidade.

\subsection{Medidas saturadas no cristal de rubi}

Apesar do tratamento desenvolvido por Oliveira et al [18] ter sido introduzido em 1996, foram poucos os trabalhos que utilizaram esta abordagem. Assim, o objetivo principal deste capítulo é enfatizar a importância do efeito da saturação sobre os parâmetros obtidos utilizando a técnica de zscan e obter seus valores corretos. Para isto, utilizamos um material amplamente conhecido, o rubi, como forma de validar nossos resultados. O cristal usado nas nossas medidas tem 1,5 mm de espessura, com uma concentração de $1,9 \times 10^{19}$ íons $/ \mathrm{cm}^{3}$ e foi cortado de maneira tal que o eixo ótico está ao longo do cristal de modo a permanecer sempre perpendicular à polarização incidente. Para excitação foram utilizadas as linhas de 457, 476, 488, 501, 514 e 528 nm do laser de $\mathrm{Ar}^{+}$.

\subsubsection{Análise do efeito da saturação sobre as medidas transientes}

Como para um sistema de três níveis a população do estado excitado é dada por:

$$
N_{e x}=N_{t} \frac{S}{1+S}\left(1-e^{-t / \tau}\right)
$$

com $\tau$ dado por

$$
\tau^{-1}=\tau_{0}^{-1}(1+S)
$$


Desta forma, pode-se usar a relação entre o inverso do tempo de resposta da não linearidade com a intensidade incidente, Equação (4-5, para se obter a intensidade de saturação no comprimento de onda de bombeio, $I_{S}$, e o tempo de vida do nível emissor do $\mathrm{Cr}^{3+}, \tau_{0}$. Isto pode ser feito pois a intensidade da luz transmitida pela amostra é proporcional à população do estado excitado, $N_{e x}$. Assim, usando um chopper para modular a luz incidente é possível obter o comportamento transiente de $N_{e x}$. Inicialmente, em t = 0 a pá do chopper bloqueia o feixe incidente e toda a população está no estado fundamental. Quando o chopper permite a passagem da luz, o estado excitado é populado seguindo o comportamento dado pela Equação (4-4, e para $t>>\tau_{0}$ atinge seu estado estacionário. Este comportamento pode ser ajustado por um decaimento exponencial, do tipo $1-e^{-t / \tau}$, para se obter o tempo de resposta da não linearidade em uma dada intensidade incidente. Esta técnica, denominada medida de refração não linear transiente, foi introduzida por Pilla et al [19] em 1997, e será utilizada para obter os valores de $I_{S}$ e $\tau_{0}$ nos diversos comprimentos de onda usados neste trabalho. Na Figura 4.2 temos dois transientes típicos para diferentes potências, em $528 \mathrm{~nm}$. Do ajuste das curvas pode-se notar a diminuição em $\tau$ com o aumento da potência.

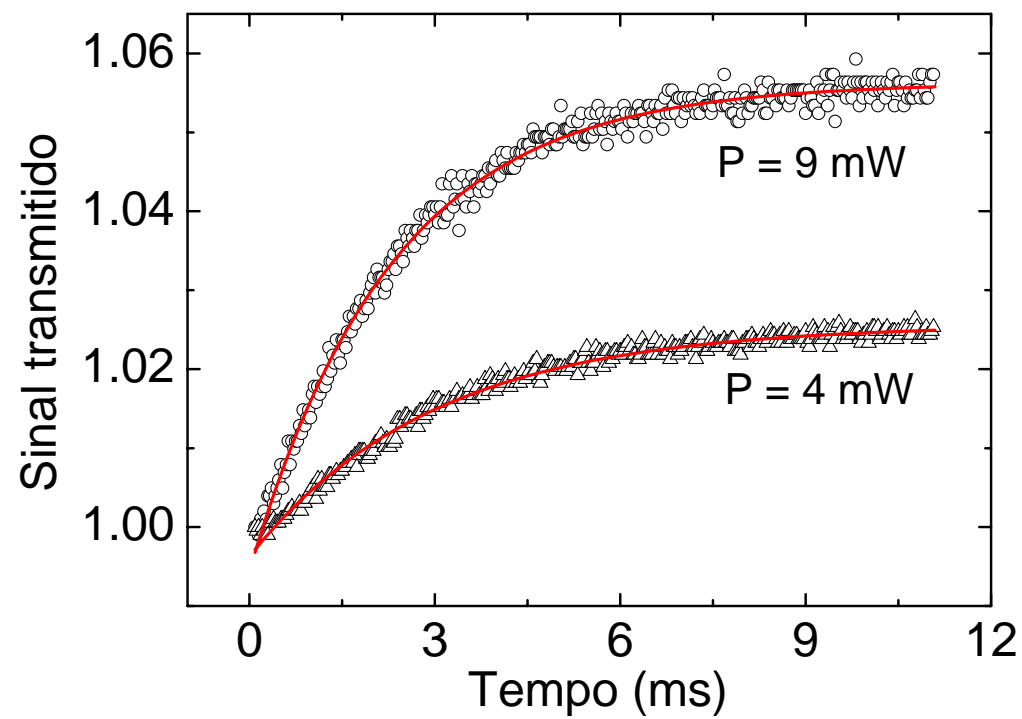

Figura 4.2 - Sinal transiente transmitido pela amostra de rubi, para duas diferentes potências. Para $P=9 \mathrm{~mW}$ foi obtido $\tau=2,31 \mathrm{~ms}$ e para $P=4 \mathrm{~mW}$ foi obtido $\tau=2,98 \mathrm{~ms}$. 
A Figura 4.3 mostra o resultado de $\tau^{-1} \times I$ para $\lambda=528 \mathrm{~nm}$. Esta figura foi ajustada com a Equação (4-5, fornecendo $\tau_{0}=(3,4 \pm 0,2)$ ms e $I_{S}=(1,0 \pm 0,1) \mathrm{KW} / \mathrm{cm}^{2}$. Este valor para $I_{S}$ foi o menor resultado encontrado dentre os comprimentos de onda utilizados. No entanto, este comportamento já era esperado visto que a amostra foi excitada bem próximo ao centro da banda ${ }^{4} \mathrm{~T}_{2}$, onde a absorção é maior. Os resultados para todos os comprimentos de onda estão listados na Tabela 4-1.

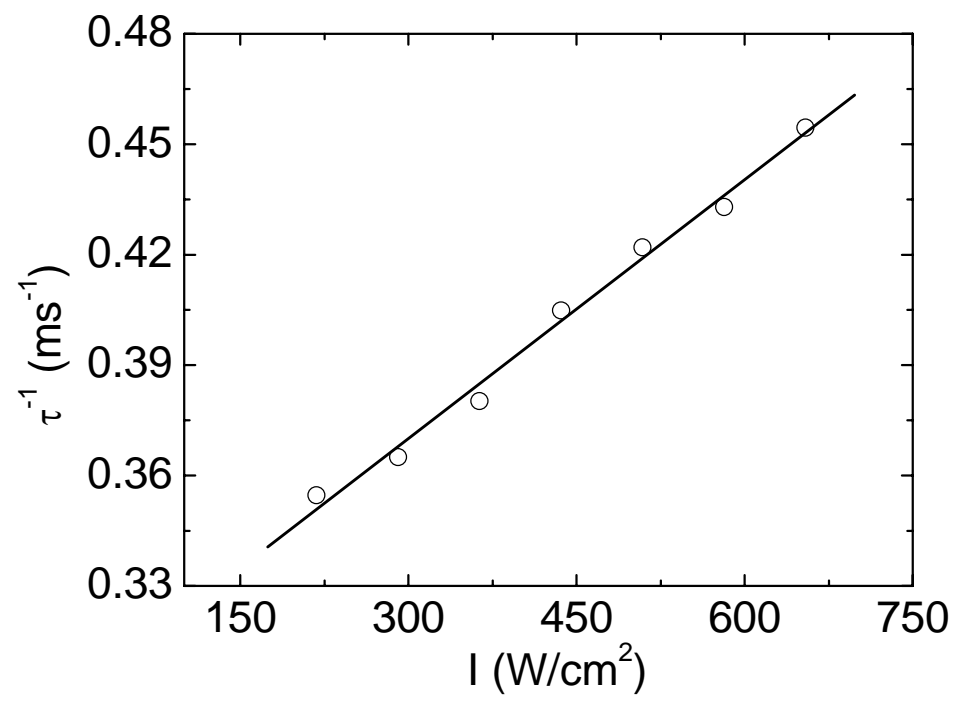

Figura 4.3 - Inverso do tempo de respostada não linearidade como função da intensidade incidente. Neste gráfico temos $\lambda=528 \mathrm{~nm}, \tau_{0}=(3,4 \pm 0,2)$ ms e $I_{S}=(1,0 \pm 0,1) \mathrm{KW} / \mathrm{cm}^{2}$, onde os valores para $\tau_{0}$ e $I_{S}$ foram obtidos à partir da Equação (4-5.

Tabela 4-1 - Tempo de vida, intensidade de saturação e coeficiente de absorção para os comprimentos de onda do $\mathrm{Ar}^{+}$. $I_{S}{ }^{*}$ é o valor calculado de $I_{S}$.

\begin{tabular}{|c|c|c|c|c|}
\hline $\begin{array}{c}\text { Comprimento de onda } \\
(\mathrm{nm})\end{array}$ & $\begin{array}{c}\tau_{0} \\
(\mathrm{~ms})\end{array}$ & $\begin{array}{c}I_{S} \\
\left(\mathrm{KW} / \mathrm{cm}^{2}\right)\end{array}$ & $\begin{array}{c}I_{S}{ }^{*} \\
\left(\mathrm{KW} / \mathrm{cm}^{2}\right)\end{array}$ & $\begin{array}{c}\alpha_{a b s} \\
\left(\mathrm{~cm}^{-1}\right)\end{array}$ \\
\hline 528 & $3,4 \pm 0,2$ & $1,0 \pm 0,1$ & 1,1 & 1,05 \\
\hline 514 & $3,7 \pm 0,3$ & $1,4 \pm 0,2$ & 1,5 & 0,75 \\
\hline 501 & $3,7 \pm 0,2$ & $2,5 \pm 0,1$ & 2,6 & 0,52 \\
\hline 488 & $3,5 \pm 0,1$ & $5,7 \pm 0,3$ & 4,2 & 0,30 \\
\hline 476 & $3,4 \pm 0,3$ & $15 \pm 2$ & 6,2 & 0,21 \\
\hline 457 & $3,5 \pm 0,2$ & $8,6 \pm 0,5$ & 5,2 & 0,31 \\
\hline
\end{tabular}


A comparação entre as intensidades de saturação obtidas, $I_{S}$, e calculadas, $I_{S}{ }^{*}$, para todos os comprimentos de onda mostra que elas concordam razoavelmente bem até $501 \mathrm{~nm}$. Após este valor começa a haver uma divergência entre estes resultados. Uma possível causa para esta discrepância pode estar na eficiência quântica de bombeamento $\eta_{p}$, que é a fração de íons que absorvem um fóton de bombeamento e atinge o estado excitado. Esta eficiência é considerada como igual a 100\% na taxa de bombeamento do estado excitado, $\eta_{\mathrm{p}} \sigma \mathrm{I} / \mathrm{h} v$, Equação 3-1. Entretanto, se o cristal apresentar impurezas que absorvam na freqüência de excitação $v$, esta taxa tende a diminuir. Assim, podemos escrever a intensidade de saturação como $I_{S}=\frac{h v}{\sigma \tau \eta_{p}}$, de modo que se $\eta_{\mathrm{p}}$ não for constante no intervalo de comprimento de onda estudado, deve-se ver uma variação na intensidade de saturação, como aquela da Tabela 4-1. O comportamento qualitativo de $\eta_{\mathrm{p}}$ como função do comprimento de onda pode ser obtido tomando a razão entre $I_{S}{ }^{*} / I_{S}$, como pode ser vista na Figura 4.4. Note que o espectro de $\eta_{\mathrm{p}}$ segue o espectro de absorção, Figura A-1. Como as causas da variação de $\eta_{\mathrm{p}}$ ainda não estão esclarecidas, mais medidas em função de $\lambda$ são necessárias para esclarecer este ponto.

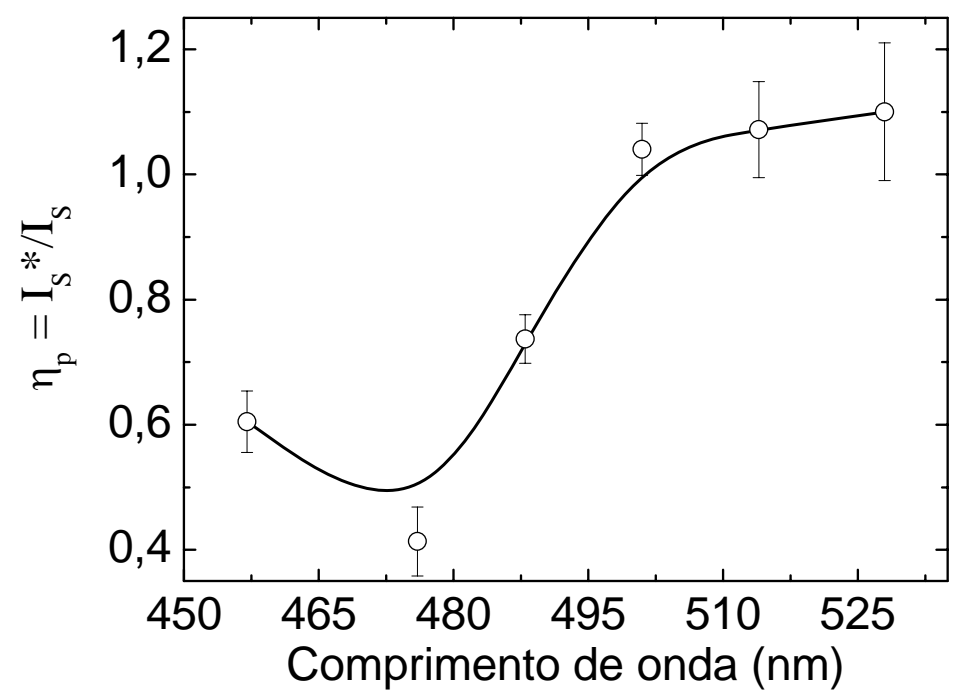

Figura 4.4 - Comportamento de $I_{S}{ }^{*} / I_{S}$ em função de $\lambda$. este comportamento é proporcional à $\eta_{\mathrm{p}}$. A linha cheia é um guia para os olhos. 
A Figura 4.5 mostra os pontos experimentais obtidos nas medidas transientes normalizados por $\tau_{0}$, para os comprimentos de onda estudados, como função do parâmetro de saturação $S$. Como esperado todas as curvas apresentam o mesmo comportamento, enfatizando a generalidade da (4-5. Os resultados obtidos nesta parte do trabalho são fundamentais para a análise subseqüente, pois esta análise já presume o conhecimento prévio da intensidade de saturação, Equações (4-2 e (4-3.

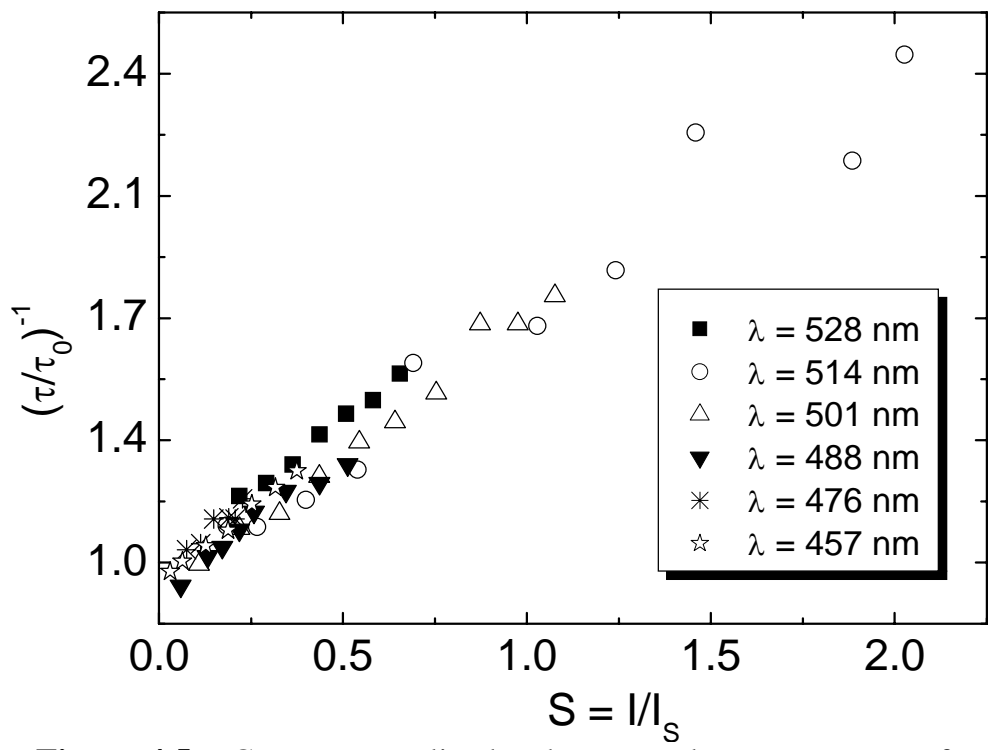

Figura 4.5 - Curvas normalizadas do tempo de resposta como função do parâmetro de saturação, para os comprimentos de onda estudados.

Os resultados apresentados acima foram obtidos num regime de saturação onde o decaimento transiente da transmitância é exponencial. No entanto, para maiores valores de $S$ foi observada numericamente uma mudança na forma de decaimento, deixando este de ser exponencial. Para comprovar a previsão teórica bombeamos o cristal de rubi até o regime de $S>10$, para o comprimento de onda de menor intensidade de saturação, $\lambda=528 \mathrm{~nm}$. A Figura 4.6 mostra os resultados experimentais, (a), assim como aquele previsto numericamente, (b). Pode-se perceber uma excelente concordância entre os dados teóricos e experimentais. 

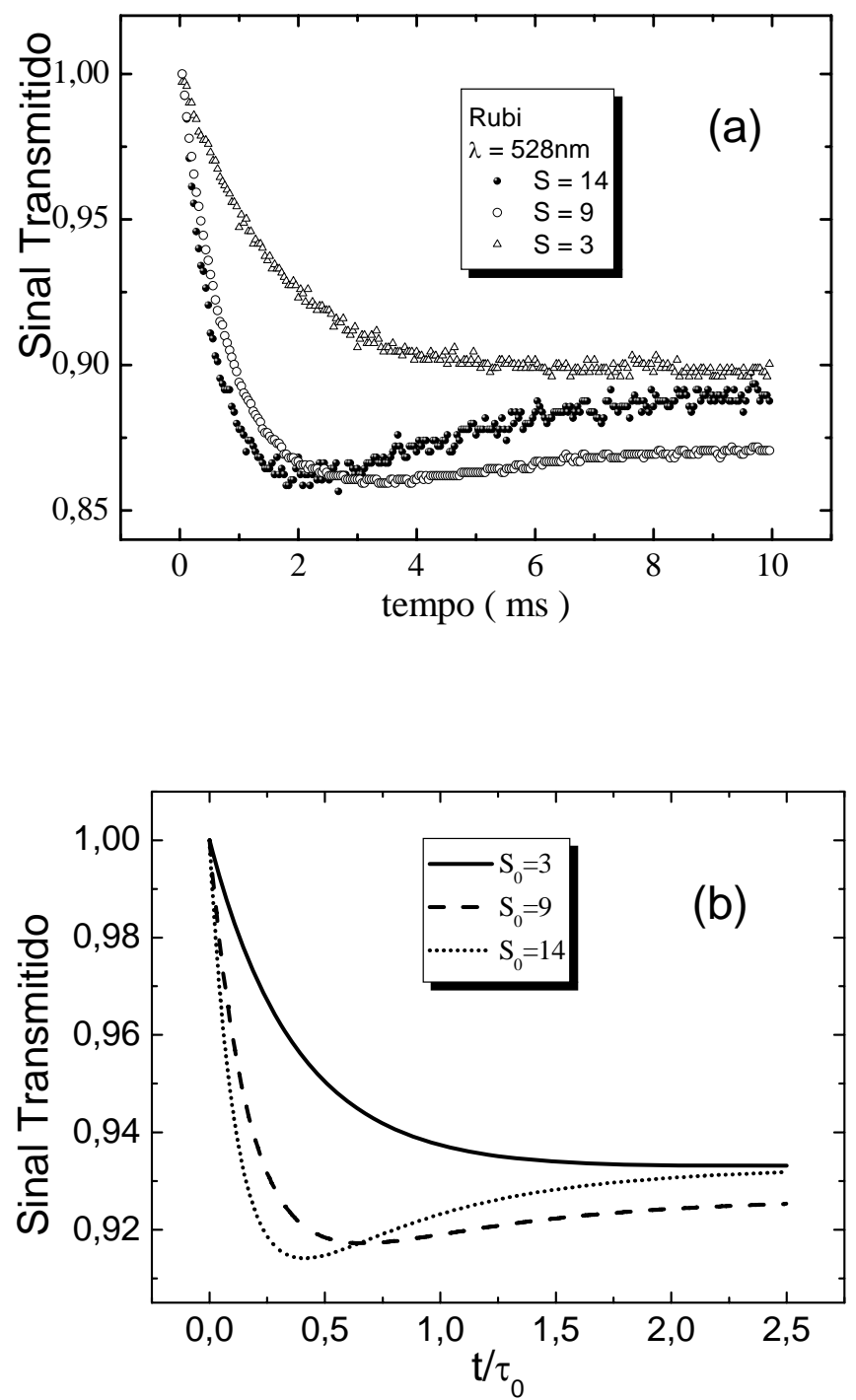

Figura 4.6 - Decaimento temporal da luz transmitida pela amostra. (a) resultado experimental e (b) simulação numérica. Dados experimentais para $\lambda=528 \mathrm{~nm}$.

Nesta medida a amostra foi colocada na posição de vale da curva de z-scan, onde a variação na transmitância é máxima para a fenda fechada. Foi visto que a partir de $S=8$ o decaimento deixa de ser exponencial, enquanto que para $S<8$ ele volta a ser exponencial. Isto acontece porque com o aumento da intensidade, o tempo característico $\tau$ torna-se cada vez mais dependente da coordenada radial da amostra através de $I(r)$, Equação (4-5. Assim, o sinal transmitido contém diferentes contribuições temporais para diferentes posições radiais. Logo, o sinal apresentado na Figura 4.6 deve ser atribuído a 
um efeito de interferência entre estas diferentes componentes temporais. Isto leva, então, ao comportamento não exponencial para o decaimento da transmitância visto na figura. Este resultado limita a aplicabilidade da técnica de refração não linear transiente a valores de S não muito maiores que 1. Para valores mais altos, este efeito de interferência pode levar a resultados errôneos de $\tau_{0}$ e $I_{S}$.

\subsection{2. $\quad$ o efeito de alargamento na curva de z-scan induzido pela saturação}

Inicialmente foram realizadas simulações usando a integral de difração para verificar o efeito da saturação sobre a curva de z-scan [20]. Através desta técnica pode-se aplicar o princípio de Huygens para um campo elétrico na saída da amostra e calcular o seu padrão espacial num plano de observação,

distante da amostra. $\mathrm{O}$ campo elétrico após a amostra pode ser expresso como $E=E_{0} e^{-i \Delta \phi}$, onde $E_{0}$ é a onda plana incidente e $\Delta \phi=(2 \pi / \lambda) L_{e f} \Delta n(r)$ é a diferença de fase não linear induzida por este campo, onde o efeito da saturação aparece em $\Delta n(r)$ como visto na Equação (4-1. Desta forma, usando esta diferença de fase saturada na integral de difração podemos descrever numericamente o comportamento da luz transmitida pela amostra.

A Figura 4.7 mostra diversas curvas teóricas de z-scan com $\Delta \phi=0,1$ para diferentes parâmetros de saturação. Na mesma figura podemos ver o caso não saturado para a mesma variação de fase. Note que o efeito inicial da saturação é diminuir a amplitude da curva, mas à medida que $S$ aumenta pode-se notar um alargamento na distância entre o pico e o vale da curva, $\Delta z_{p v}$. 


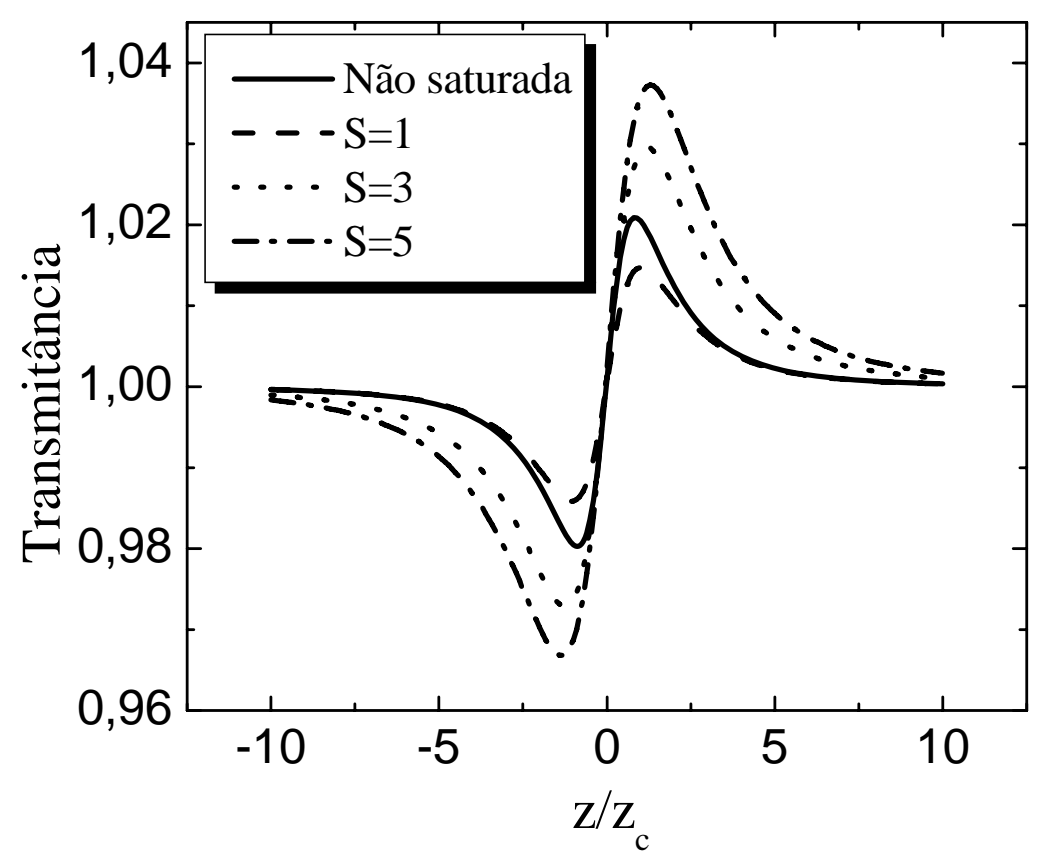

Figura 4.7 - Curvas teóricas de z-scan obtidas através da integral de difração. Nesta figura percebe-se um ligeiro aumento de $\Delta z_{p v}$ quando $S$ aumenta.

Graficamos então $\Delta z_{p v}$ normalizado por $z_{c}$ como função de $S$, como pode ser visto na Figura 4.8, e ajustamos os pontos com a expressão empírica

$$
\frac{\Delta z_{p v}}{z_{c}}=\varepsilon\left(1+a S^{b}\right)
$$

onde os valores encontrados foram $\varepsilon=1,7, a=0,2$ e $b=0,7$. Assim, no limite de $S \rightarrow 0$, a expressão $\Delta z_{p v}=1,7 z_{c}$ é recuperada. É importante enfatizar que cada ponto desta curva foi obtido através de simulação numérica, e os resultados foram ajustados com a Equação (4-6. 


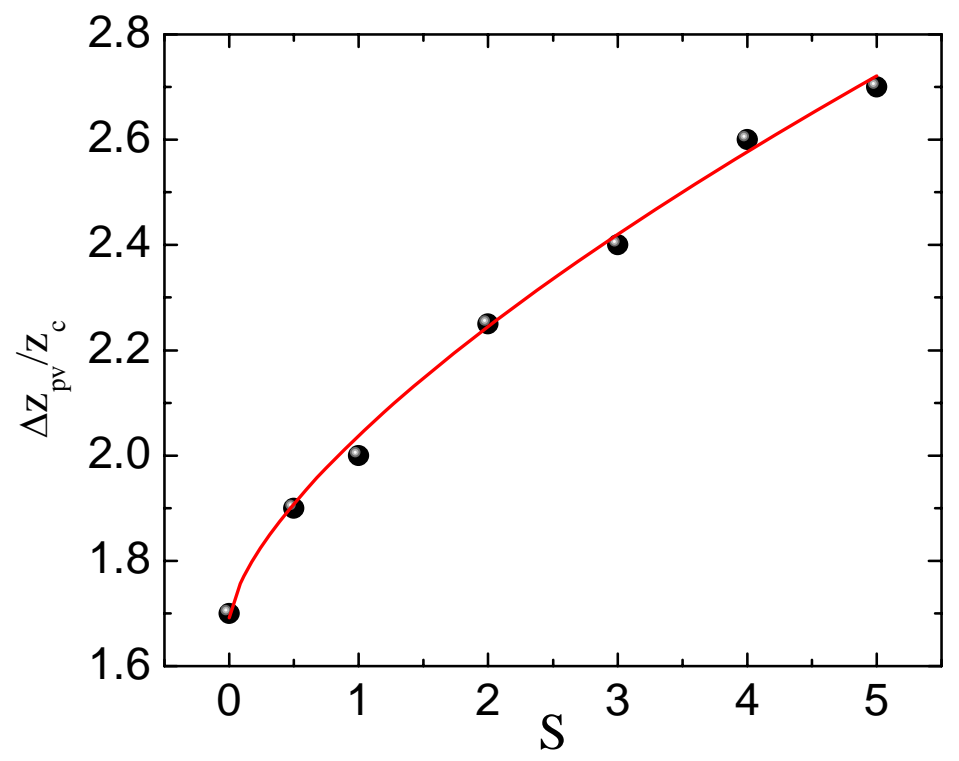

Figura 4.8 - Efeito do alargamento de $\Delta z_{p v}$ previsto das simulações numéricas. Estes dados foram obtidos da Figura 4.7. Aqui $z_{c}=1 \mathrm{~cm}$.

Na Figura 4.9 vemos a verificação experimental do efeito de alargamento da curva refrativa de Z-scan para o cristal de rubi excitado em $528 \mathrm{~nm}$.

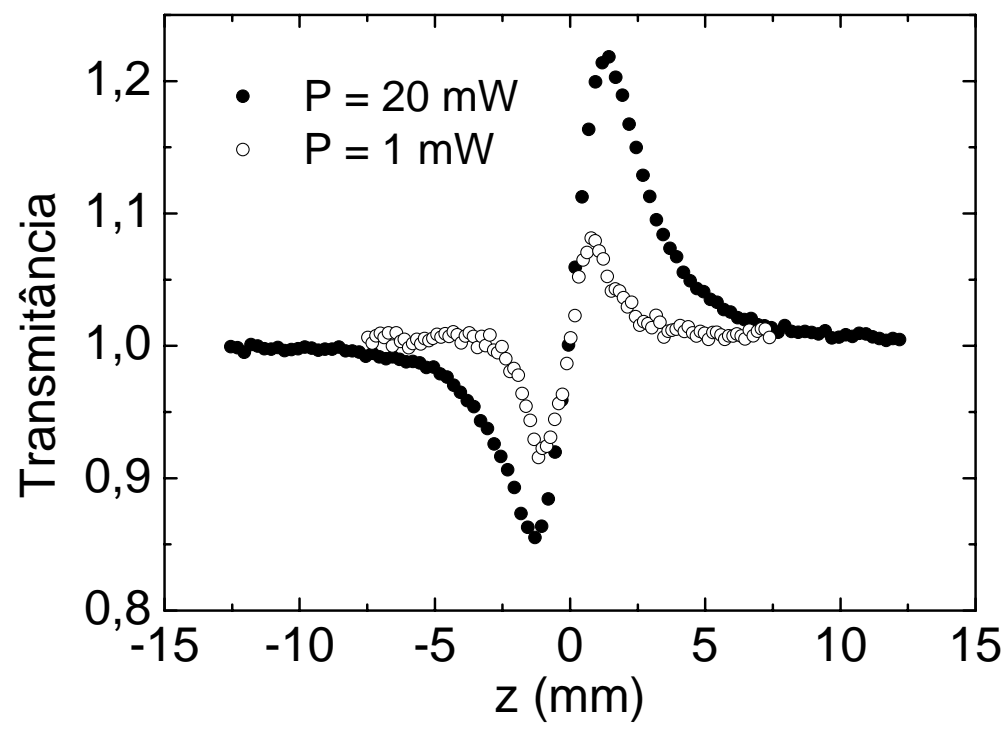

Figura 4.9 - Curvas de $z$-scan saturadas em $\lambda=528 \mathrm{~nm}$. Note que existe um leve alargamento das curvas com o aumento da potência incidente.

Como previsto teoricamente existe um leve alargamento da curva de z-scan quando a potência incidente aumenta de $1 \mathrm{~mW}$ para $20 \mathrm{~mW}$. Diversas curvas para diferentes potências foram obtidas, e a distância de pico a vale foi então graficada como função do parâmetro de excitação, Figura 4.10. 
Usando $I_{S}=1 \mathrm{KW} / \mathrm{cm}^{2}$ e $z_{c}=0,81 \mathrm{~cm}$ foram ajustados os pontos experimentais com a (4-6 de onde se obteve $\varepsilon=(1,72 \pm 0,01), a=(0,19 \pm 0,01)$ e $b=(0,7 \pm 0,03)$, o que concorda muito bem com os valores obtidos anteriormente através de simulações. Como esperado, para $S<<1$ a relação usual $\Delta Z_{p v} \sim 1,7 \mathrm{Z}_{c}$ foi recuperada. É importante destacar que os valores calculados para as constantes $\varepsilon$, $a$ e $b$ independem de características do material, permitindo que a Equação (4-6 possa ser aplicada em diferentes sistemas como vapores atômicos, polímeros, corantes, etc.

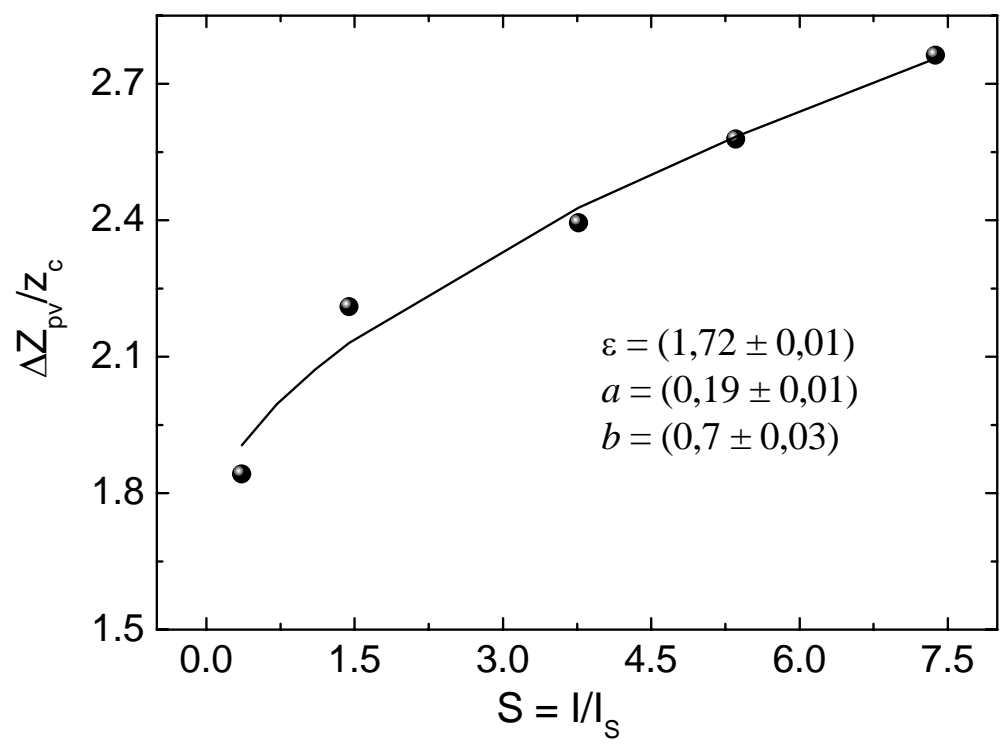

Figura 4.10 - Comportamento experimental da distância de pico a vale da curva de z-scan como função do parâmetro de saturação, $S$, para $\lambda=528 \mathrm{~nm}$, $I_{S}=1 \mathrm{KW} / \mathrm{cm}^{2}$ e $z_{\mathrm{c}}=0,81 \mathrm{~cm}$. os dados experimentais foram ajustados com a Equação (4-6.

Este alargamento pode ser explicado da seguinte maneira: o efeito da saturação, como visto na Figura 4.1, causa um achatamento no perfil de índice de refração, que por sua vez leva a um aumento no comprimento focal da lente induzida. Logo, para $z<0$ um foco não linear mais longo fará com que o feixe chegue menos espalhado no detector, movendo a posição de vale para antes de sua posição inicial. Da mesma forma, para $z>0$ o máximo da curva é agora alcançado mais próximo do detector. Logo, este maior comprimento focal provoca um alargamento entre o pico e o vale da curva. 
Este alargamento poderia vir a ser confundido com aquele devido ao efeito térmico, onde $\Delta Z_{p v} / Z_{c} \sim 3,4[21,22]$. No entanto, para o rubi a difusividade térmica é elevada, $D=0,13 \mathrm{~cm}^{2} / \mathrm{s}$ [23] (compare com a Tabela 2-1), o que torna a não linearidade térmica desprezível diante da eletrônica. Logo, o efeito de alargamento, neste caso, deve ser atribuído apenas ao efeito eletrônico de saturação. Assim, em um material onde se desconhece a magnitude das contribuições térmica e eletrônica, é necessário obter a curva de $z$-scan à baixa intensidade e verificar a relação entre $\Delta Z_{p v} \operatorname{com} z_{c}$ para se ter uma noção de qual a maior contribuição.

Finalmente, a Equação (4-6 pode ser considerada uma generalização de relação entre $\Delta Z_{p v}$ com $z_{c}$ e deve ser usada para obter o valor correto de $z_{c}$, e consequentemente de $w_{0}$, sempre que a medida de z-scan for realizada no regime em que há saturação da não linearidade. Este dado é muito importante pois através dele se determina a intensidade incidente na amostra, o que afeta todos os resultados.

\subsubsection{Determinação dos parâmetros não lineares no rubi}

Duas curvas típicas de z-scan, uma saturada (experimental) e outra não saturada (simulação), podem ser vistas na Figura 4.11 para $\lambda=501 \mathrm{~nm}$ e $S=0,5$. Comparando com a Figura 4.7 podemos ver experimentalmente uma diminuição na amplitude da curva para o caso saturado. Ajustando estas duas curvas com a Equação 3-23 leva a uma diferença de aproximadamente 30\% no valor obtido para $\Delta \phi$. Isto implica que o valor obtido para $n_{2}$ ' corre o risco de ser determinado com um erro muito grande devido à saturação. Por conseguinte, o valor de $\Delta \alpha$ também seria erroneamente estimado. Analogamente, o efeito da saturação também se faz sentir sobre a curva da absorção saturada (fenda aberta), e as mesmas considerações podem ser feitas para $n_{2} ”$ e $\Delta \sigma$. 


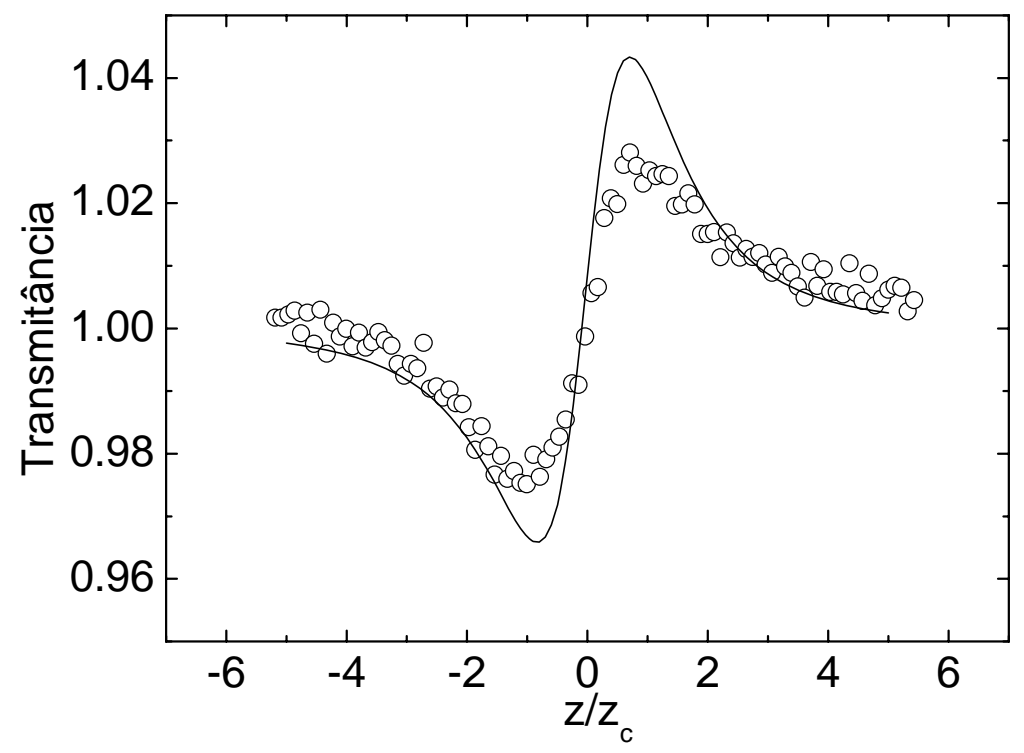

Figura 4.11 - Círculos: curva de z-scan saturada em rubi, onde $S=0,5$ e $\lambda=501$ $\mathrm{nm}$. Linha cheia: simulação numérica da mesma medida, mas desprezando o efeito da saturação.

Após estas medidas preliminares, foram então realizadas várias medidas de z-scan nos comprimentos de onda do laser de $\mathrm{Ar}^{+}$. O objetivo destas medidas foi verificar o efeito da saturação sobre a curva de z-scan e obter os valores corretos de $n_{2}, \Delta \alpha$ e $\Delta \sigma$ para o cristal de rubi. A Figura 4.12 mostra o comportamento de $\Delta T_{p v}$ em função da intensidade incidente. Os ajustes destas curvas foram feitos com a Equação (4-2 com os valores de $I_{S}$ da Tabela 4-1. Destes ajustes foram obtidos $n_{2}$ ' para cada um dos comprimentos de onda. A seguir foram calculados os valores de $\Delta \alpha$, através da Equação 3-12. 


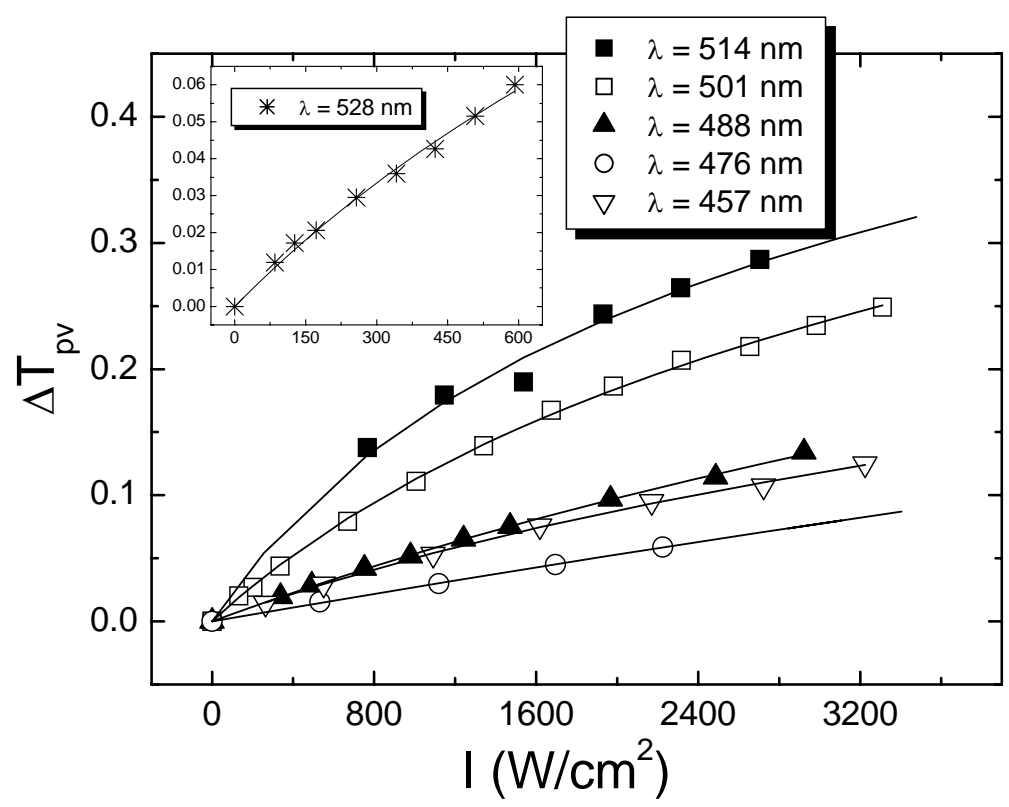

Figura 4.12 - Variação da transmitância entre o pico e o vale da curva de zscan (fenda fechada) num cristal de rubi como função da intensidade incidente, para os comprimentos de onda do laser de $\mathrm{Ar}^{+}$. As linhas cheias representam ajustes feitos com a Equação (4-2.

Comparando esta figura com a Tabela 4-1, vemos que quem domina a amplitude da curva é a intensidade de saturação. Isto pode ser compreendido lembrando que $\Delta T_{p v} \propto \Delta \phi \propto \frac{\Delta \alpha}{I_{S}}$. Por isso, à medida que $I_{S}$ aumenta as curvas vão diminuindo em amplitude. É interessante notar que como $I_{S}$ é menor para 457 nm do que para 476 nm, o primeiro aparece acima do último na Figura 4.12. Os resultados encontrados em 514 nm novamente estão em bom acordo com os da literatura, Tabela 4-2, reforçando a confiabilidade de nossos resultados em outros comprimentos de onda. Foi visto que embora exista uma diferença de uma ordem de magnitude nos valores de $n_{2}$ ' e $n_{2}$ " para este comprimento de onda, como foi algumas vezes enfatizado na literatura [11], esta relação não se mantém para os demais. Na verdade, em $528 \mathrm{~nm}$, próximo ao centro da banda ${ }^{4} \mathrm{~T}_{2}$ onde a absorção medida foi a maior, as partes real e imaginária de $n_{2}$ tem a mesma magnitude. Por outro lado em 476 nm esta diferença aumenta para duas ordens de magnitude, pois como estamos bombeando entre as 
bandas ${ }^{4} \mathrm{~T}_{1} \mathrm{e}{ }^{4} \mathrm{~T}_{2}$, o coeficiente de absorção é o menor dentre os medidos ( $c f$ Figura A-1), diminuindo assim os efeitos absortivos. Os valores encontrados para $\Delta \alpha$ estão próximos entre si, indicando que sua maior contribuição deve vir da banda de transferência de carga, como descrito por Powell et al [24] para outros cristais dopados com $\mathrm{Cr}^{3+}$. Na média, adicionando os resultados da literatura, temos que $\Delta \alpha=1,9 \times 10^{-25} \mathrm{~cm}^{3}$ para o rubi.

Com relação à absorção não linear, pode-se ver uma mudança em seu caráter de saturada para saturada reversa ${ }^{2}$ com a diminuição do comprimento de onda. A Figura 4.13 mostra este comportamento para os comprimentos de onda de $501 \mathrm{~nm}$ e $457 \mathrm{~nm}$. Isto demonstra que o rubi pode se comportar como um meio absorvedor direto ou reverso dependendo da energia da excitação.

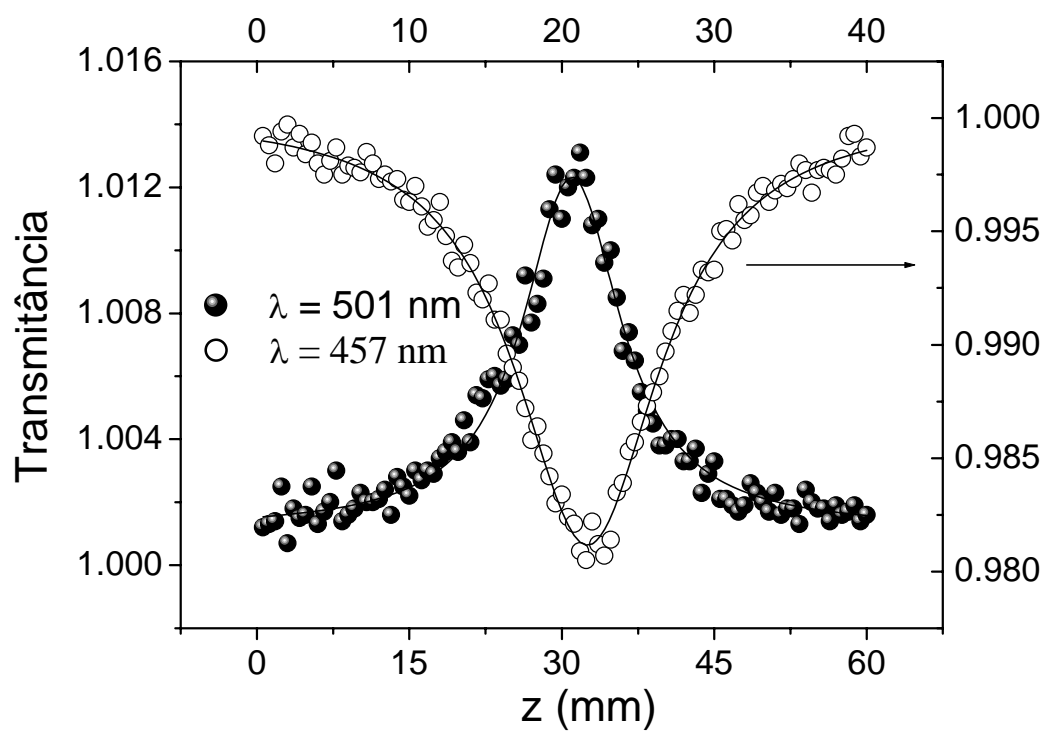

Figura 4.13- Mudança no caráter da absorção de saturável (501 nm) para saturável reverso (457 nm) num cristal de rubi com o aumento da energia de excitação.

Da mesma forma que para o caso refrativo, a variação da transmitância para a fenda aberta foi também graficada como função da intensidade incidente, Figura 4.14. Ajustando então as curvas com a

\footnotetext{
${ }^{2} \mathrm{Na}$ absorção saturada a transmissão do sinal cresce com o aumento da intensidade, devido à diminuição da absorção, pois existem menos elétrons no estado fundamental para serem absorvidos. A absorção saturada reversa tem o comportamento oposto, diminuindo a transmitância com o aumento da intensidade.
} 
Equação (4-3 e usando a seguir a Equação 3-13 foram então obtidos $n_{2}$ ” e $\Delta \sigma$, respectivamente, Tabela 4-2.

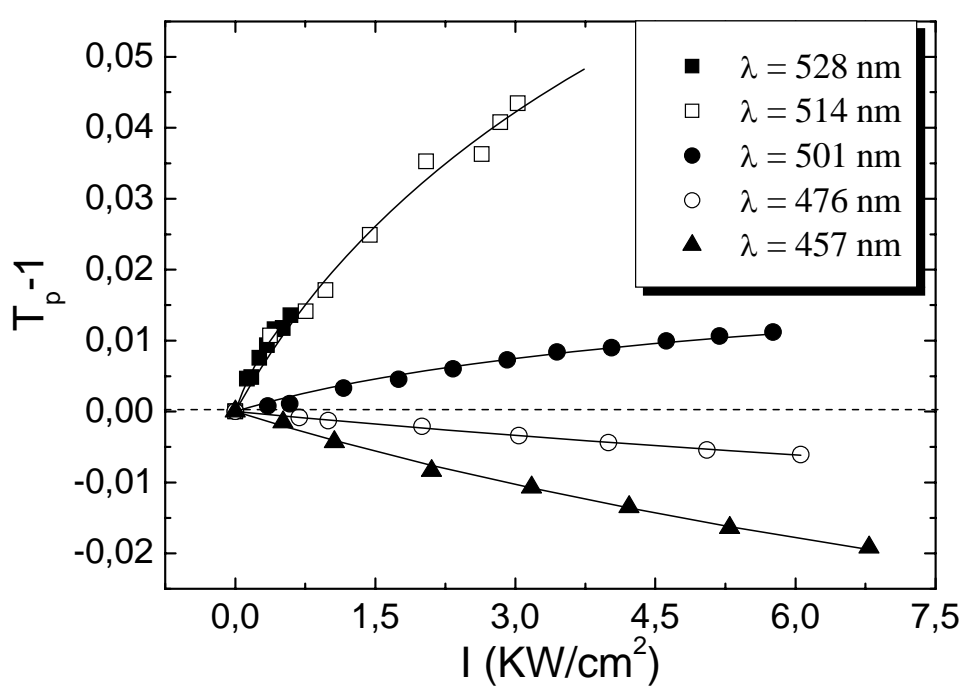

Figura 4.14 - Máxima variação da transmitância para a fenda aberta num cristal de rubi como função da intensidade incidente. As linhas cheias são ajuste feitos com a Equação (4-3. Acima da linha tracejada estão os casos que apresentaram absorção saturada e abaixo os que apresentaram absorção saturada reversa.

Esta mudança no caráter absorvedor deve-se à mudança na absorção de estado excitado (AEE) do nível ${ }^{2} E$ para estados mais altos de energia, em função do comprimento de onda. Kushida [25] mostrou, em um cristal de rubi, que para $\lambda>500 \mathrm{~nm}$ a absorção do nível fundamental é maior que a do estado excitado $(\Delta \sigma>0)$ e que abaixo deste comprimento de onda acontece o reverso $(\Delta \sigma<0)$. De fato, para $\lambda=488 \mathrm{~nm}$ não foi possível obter um valor para $\Delta \sigma$ com precisão $(\Delta \sigma=0.0 \pm 0.1$ ), pois não conseguimos ver saturação da absorção, i. e., a variação da transmitância foi praticamente zero para a fenda aberta e por isso não aparece na Figura 4.14. Desta maneira concluímos que para $\lambda=488 \mathrm{~nm}$ teremos $\sigma_{e} \sim \sigma_{g}=1,57 \times 10^{-20} \mathrm{~cm}^{2}$, calculado usando $\sigma_{g}=\alpha_{a b s} / N_{t}$. 
Tabela 4-2 - Parâmetros não lineares obtidos à partir das medidas saturadas de z-scan.

\begin{tabular}{|c|c|c|c|c|c|}
\hline $\begin{array}{c}\text { Comprimento } \\
\text { de onda } \\
(\mathrm{nm})\end{array}$ & $\begin{array}{c}n_{2}^{\prime} \\
\left(\times 10^{-9} \mathrm{~cm}^{2} / \mathrm{W}\right)\end{array}$ & $\begin{array}{c}n_{2} ” \\
\left(\times 10^{-9} \mathrm{~cm}^{2} / \mathrm{W}\right)\end{array}$ & $\begin{array}{c}\Delta \alpha \\
\left(\times 10^{-25} \mathrm{~cm}^{3}\right)\end{array}$ & $\begin{array}{c}\Delta \sigma \\
\left(\mathbf{x 1 0}^{-20} \mathrm{~cm}^{2}\right)\end{array}$ & Ref. \\
\hline 457 & 3.5 & 0.1 & 1.6 & 1.4 & $a$ \\
\hline 476 & 3.0 & 0.034 & 1.7 & 0.53 & $a$ \\
\hline 488 & 5.0 & - & 1.8 & - & $a$ \\
\hline 501 & 11.9 & -0.19 & 2.4 & -0.7 & $a$ \\
\hline \multirow[t]{4}{*}{514} & 16.3 & -0.10 & 2.1 & -1.7 & $a$ \\
\hline & 15.5 & -0.6 & 2.2 & & [18] \\
\hline & 12.5 & & 1.7 & & [5] \\
\hline & 16 & 0.96 & 2.0 & & [26] \\
\hline 528 & 21.7 & -1.5 & 2.2 & -5 & $a$ \\
\hline 633 & 21.6 & & - & & [26] \\
\hline 694 & 4 & 0.25 & 1.6 & & [27] \\
\hline
\end{tabular}

Uma nota importante sobre as propriedades do rubi é que ele pode ser um bom candidato para apresentar ao mesmo tempo propagação sub $(v<<c)$ e super-luminal $(v>c$, onde $v$ é a velocidade da luz no meio). Bigelow et al[28-30] já demonstraram propagação sub-luminal no rubi, e ambos sub e super-luminal na alexandrita. A descrição teórica deste fenômeno foi dada mais recentemente por Agarwal e Dey [31], que desenvolveram um modelo que apresenta propagação sub-luminal e superluminal para sistema que exibem absorção direta e reversa, respectivamente. Logo, nós acreditamos que usando comprimentos de onda menor que $488 \mathrm{~nm}$ seja possível alcançar velocidade super-luminal também no rubi. 


\subsection{Conclusões do capítulo}

Neste Capítulo nós demonstramos numericamente e experimentalmente os efeitos da saturação sobre a curva de z-scan, num cristal de rubi. Nossas medidas transientes mostraram como a saturação afeta o decaimento da luz transmitida, levando a um decaimento não exponencial para altos valores do parâmetro de saturação. Usando o modelo de Oliveira et al, nós obtivemos os valores de $n_{2}, \Delta \alpha$ e $\Delta \sigma$ à partir do comportamento de $\Delta T_{p v}$ e $T_{p}$-1 com a intensidade incidente. Esta medida foi feita para diversos comprimentos de onda de excitação. Este resultado é particularmente importante para o desenvolvimento dos sistemas laser de alta potência, pois o conhecimento do comportamento das propriedades óticas não lineares neste regime é fundamental em seu design. O alargamento previsto e observado experimentalmente para $\Delta Z_{p v}$ pôde ser explicado pelo aumento do foco não linear devido à saturação. Finalmente a mudança no comportamento do rubi de absorvedor saturável para saturável reverso, aumentando a energia de excitação, foi explicada como absorção de estado excitado do nível

${ }^{2}$ E para níveis superiores. 


\subsection{Referências}

[1] H. J. Eichler, P. Günter, and D. W. Pohl, Laser-Induced Dynamics Gratings, Springer-Verlag, New York, 1986.

[2] Y. F. Chen, C. C. Liao, Y. P. Lan, and S. C. Wang, Determination of the Auger upconversion rate in fiber-coupled diode end-pumped Nd : YAG and Nd : YVO4 crystals, App. Phys. B 70 (2000) 487-490.

[3] A. M. Ghazzawi, J. K. Tyminski, R. C. Powell, and J. C. Walling, 4-Wave Mixing in Alexandrite Crystals, Phys. Rev. B 30 (1984) 7182-7186.

[4] A. Suchocki, G. D. Gilliland, and R. C. Powell, 4-Wave-Mixing Measurements of Energy Migration and Radiationless Relaxation Processes in Alexandrite Crystals, Phys. Rev. B 35 (1987) 5830-5840.

[5] T. Catunda, J. P. Andreeta, and J. C. Castro, Differential Interferometric-Technique for the Measurement of the Nonlinear Index of Refraction of Ruby and Gdaio3-Cr-3+, App. Opt. 25 (1986) 2391-2395.

[6] I. Mcmichael, P. Yeh, and P. Beckwith, Nondegenerate 2-Wave Mixing in Ruby, Opt. Lett. 13 (1988) 500-502.

[7] S. A. Boothroyd, J. Chrostowski, and M. S. Osullivan, 2-Wave Mixing by Phase and Absorption Gratings in Saturable Absorbers, J. Opt. Soc. Am. B 6 (1989) 766-771.

[8] M. Sheik-bahae, A. A. Said, and E. W. V. Stryland, High sensitivity, single-beam $n_{2}$ measurements, Opt. Lett. 14 (1989) 955-958.

[9] P. Roussignol, D. Ricard, J. Lukasik, and C. Flytzanis, New Results on Optical-Phase Conjugation in Semiconductor-Doped Glasses, J. Opt. Soc. Am. B 4 (1987) 5-13. 
[10] T. Catunda and L. A. O. Nunes, Interference Effects in the Degenerate-Wave-Mixing Spectroscopy of Alexandrite, Phys. Rev. B 45 (1992) 10087-10090.

[11] T. Catunda and L. A. Cury, Transverse Self-Phase Modulation in Ruby and Gdalo3-Cr-+3 Crystals, J. Opt. Soc. Am. B 7 (1990) 1445-1455.

[12] T. Catunda, A. M. Cansian, and J. C. Castro, Saturation Effects in Degenerate 4-Wave-Mixing in Ruby and Gdalo3-Cr+3, J. Opt. Soc. Am. B 8 (1991) 820-823.

[13] C. F. McCormick, D. R. Solli, R. Y. Chiao, and J. M. Hickmann, Saturable nonlinear refraction in hot atomic vapor, Phys. Rev. A 69 (2004) 023804.

[14] L. Demenicis, A. S. L. Gomes, D. V. Petrov, C. B. deAraujo, C. P. deMelo, C. G. dosSantos, and R. SoutoMaior, Saturation effects in the nonlinear-optical susceptibility of poly(9hexadecylthiophene), J. Opt. Soc. Am. B 14 (1997) 609-614.

[15] M. Martinelli, S. Bian, J. R. Leite, and R. J. Horowicz, Sensitivity-enhanced reflection Z-scan by oblique incidence of a polarized beam, Appl. Phys. Lett. 72 (1998) 1427-1429.

[16] S. Bian, M. Martinelli, and R. J. Horowicz, Z-scan formula for saturable Kerr media, Opt. Comm. 172 (1999) 347-353.

[17] Y. X. Wang and M. Saffman, Z-scan formula for two-level atoms, Opt. Comm. 241 (2004) 513520.

[18] L. C. Oliveira, T. Catunda, and S. C. Zilio, Saturation effects in Z-scan measurements, Jap. J. App. Phys. 35 (1996) 2649-2652.

[19] V. Pilla, P. R. Impinnisi, and T. Catunda, Measurement of saturation intensities in ion doped solids by transient nonlinear refraction, Appl. Phys. Lett. 70 (1997) 817-819.

[20] J. W. Goodman, Introduction to Fourier optics, McGraw-Hill New York, 1996. 
[21] V. Pilla, A. A. Andrade, S. M. Lima, T. Catunda, D. A. Donatti, D. R. Vollet, and A. I. Ruiz, Spectroscopic and thermal characterization in poly(p-phenylene vinylene)/sol-gel silica sample, Opt. Mat. 24 (2003) 483-489.

[22] A. A. Andrade, E. Tenorio, T. Catunda, M. L. Baesso, A. Cassanho, and H. P. Jenssen, Discrimination between electronic and thermal contributions to the nonlinear refractive index of SrAlF5 : Cr+3, J. Opt. Soc. Am. B 16 (1999) 395-400.

[23] R. C. Powell, Physics of Solid-State Lasers Materials, Springer-Verlag, New York, 1998.

[24] R. C. Powell and S. A. Payne, Dispersion Effects in 4-Wave-Mixing Measurements of Ions in Solids, Opt. Lett. 15 (1990) 1233-1235.

[25] T. Kushida, Absorption and Emission Properties of Optically Pumped Ruby, IEEE J. Quant. Elect. Qe 2 (1966) 524-531.

[26] J. P. Bernardin, A. S. L. Gomes, J. L. Cohen, and N. M. Lawandy, Experiments and Numerical Simulations on Beam Bending and Transverse Beam Encoding by Spatial Self-Phase and Cross-Phase Modulation in Ruby, Opt. Comm. 103 (1993) 285-296.

[27] S. M. Lima, H. Jiao, L. A. O. Nunes, and T. Catunda, Nonlinear refraction spectroscopy in resonance with laser lines in solids, Opt. Lett. 27 (2002) 845-847.

[28] M. S. Bigelow, N. N. Lepeshkin, and R. W. Boyd, Superluminal and slow light propagation in a room-temperature solid, Science 301 (2003) 200-202.

[29] M. S. Bigelow, N. N. Lepeshkin, and R. W. Boyd, Observation of ultraslow light propagation in a ruby crystal at room temperature, Phys. Rev. Lett. 90 (2003) 113903.

[30] M. S. Bigelow, N. N. Lepeshkin, and R. W. Boyd, Ultra-slow and superluminal light propagation in solids at room temperature, J. Phys. Cond. Mat. 16 (2004) R1321-R1340.

[31] G. S. Agarwal and T. N. Dey, Sub-and superluminal propagation of intense pulses in media with saturated and reverse absorption, Phys. Rev. Lett. 92 (2004) 203901. 


\section{Capítulo 5}

\section{Determinação do parâmetro de upconversion Auger através da técnica de}

\section{z-scan}

A tecnologia de lasers de estado sólido avançou até o ponto onde é possível prever com confiança como será a performance de um meio ativo. No entanto, este avanço gerou novos limites para esta tecnologia. Atualmente, a construção de sistemas lasers de alta potência e a busca pela miniaturização dos lasers de estado sólido tem sido o foco de diversas pesquisas em todo o mundo [13]. Além disso, alguns cristais dopados com neodímio, por exemplo, tem atraído um grande interesse pois eles permitem a geração de luz azul através de dobramento de freqüência [4-7]. No entanto, altas eficiências de lasers neste esquema de quase três níveis $\left({ }^{4} \mathrm{~F}_{3 / 2} \rightarrow{ }^{4} \mathrm{I}_{9 / 2}\right)$ são consideravelmente mais difíceis de alcançar do que a transição mais forte em torno de $1100 \mathrm{~nm}\left({ }^{4} \mathrm{~F}_{3 / 2} \rightarrow{ }^{4} \mathrm{I}_{11 / 2}\right)$, que funciona como um sistema de 4 níveis. Os problemas com esta transição são as altas perdas por reabsorção e a baixa seção de choque de emissão. Para suplantar este problema em geral empregam-se fontes mais potentes de bombeamento, e/ou maior focalização da luz de bombeamento, estas condições propiciam o aparecimento de efeitos danosos à performance do dispositivo. Outro problema ainda a ser resolvido é melhorar a performance de cristais auto ativados. Nestes cristais os íons dopantes fazem parte da própria estrutura cristalina, de maneira que são ditos $100 \%$ dopados, de modo que apresentam alta absorção e por isso são pretendidos para miniaturização $[8,9]$. Nesta condição a distância interiônica é muito menor que nos casos onde o íon emissor entra como uma impureza. Isto faz com que os processos não radiativos de decaimento se tornem mais evidentes. Para os exemplos de aplicações citados acima um dos mais importantes processos não radiativos que influenciam a performance dos dispositivos é o de Transferência de Energia por Conversão Ascendente (Energy Transfer 
Upconversion ou upconversion Auger). Este mecanismo é responsável pelo aquecimento em lasers de estado sólido sob a condição de altas intensidades de bombeio [10,11]. Este efeito danoso incrementa a geração de calor, aumentando assim a lente térmica, e ao mesmo tempo diminui a população do nível laser superior, causando uma baixa na eficiência de emissão do nível. Em última análise este processo leva a uma queda na eficiência quântica em lasers, de forma que seu principal efeito é limitar a potência de saída dos lasers de estado sólido.

Algumas técnicas vêm sendo utilizadas a fim de caracterizar este processo, tais como o estudo do decaimento temporal da fluorescência (que deixa de ser exponencial e assume uma forma mais complexa $[12,13])$, a oscilação transiente da potência de saída do laser devido a translação do meio ativo [14], e mais recentemente espectrometria de lente térmica $[15,16]$. No entanto, estas técnicas em geral apresentam algumas inconveniências como necessidade de altas potências incidente (algumas vezes próximas ao limiar de dano do material), dificuldade de implementação ou necessidade de medidas adicionais para a determinação de parâmetros necessários. Neste ínterim o ideal seria usar uma técnica de fácil implementação, amplamente conhecida e que apresentasse requerimentos facilmente alcançáveis. Para isto o Prof. Acácio A. Andrade e colaboradores introduziram a metodologia de usar a técnica de $z$-scan para obter o parâmetro de upconversion. O objetivo deste trabalho é dar continuidade àquele inicialmente desenvolvido pelo Prof. Acácio, aplicando a técnica a diferentes materiais lasers, estudar como o comportamento transiente pode também ser utilizado para obter este parâmetro e obter através de simulações numéricas o comportamento da variação da transmitância da fenda fechada sob o efeito deste processo.

Os trabalhos deste capítulo foram realizados em colaboração com os professores Sandro M. Lima da UEMS da Universidade Estadual do Mato Grosso do Sul, Acácio A. Andrade da Universidade Estadual do Norte Fluminense e com o doutorando Carlos J. da Silva. 


\subsection{Dinâmica do processo de upconversion Auger}

A maneira como o processo de upconversion Auger acontece é bem conhecida. Inicialmente dois íons excitados que estão próximos entre si, interagem não radiativamente de modo que a energia de um deles é transferida para o outro, Figura 5-1(a). O íon que perde energia é então levado a um estado metaestável inferior, de onde decai para o estado fundamental. Já o íon que ganha energia é levado a um estado excitado superior de onde decai de volta para o nível onde estava inicialmente Figura 5-1(b). Ao final do processo resta apenas um íon no nível excitado inicial, ao mesmo tempo em que uma grande quantidade da energia absorvida que gera excitação é transformada em calor. Em geral, quanto maior a concentração de dopante e/ou maior a intensidade incidente, mais pronunciado é o efeito de upconversion Auger.

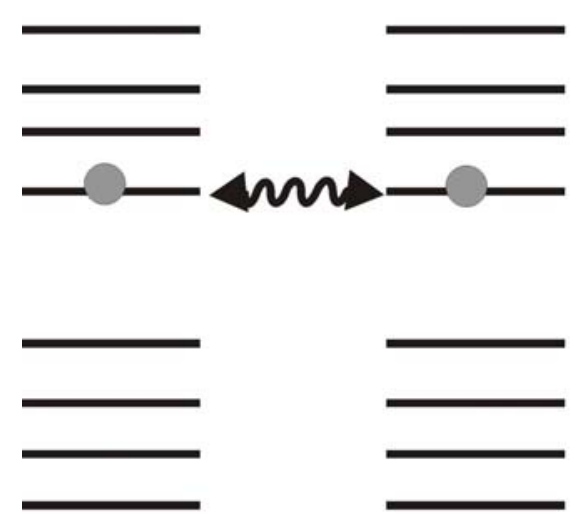

(a)
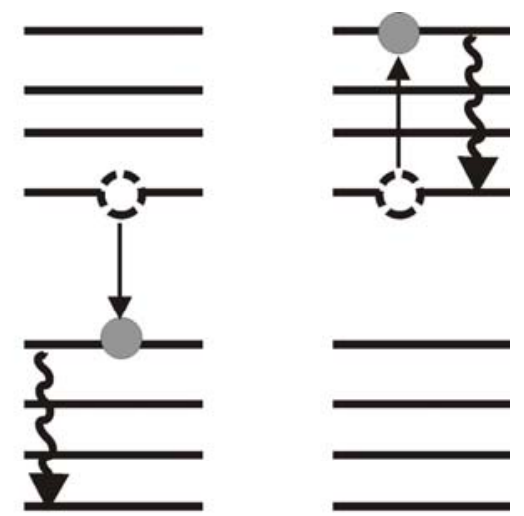

(b)

Figura 5-1 - Diagrama esquemático do processo de upconversion Auger. (a) Inicialmente dois íons no estado excitado interagem não radiativamente; (b) logo após um dele é levado a um estado excitado superior enquanto o outro vai para um estado excitado inferior. As setas onduladas indicam processos não radiativos.

Todo este processo pode ser descrito usando equações de taxa para representar as populações dos níveis de interesse, onde a forma da equação muda dependendo do nível excitado. Normalmente em materiais como $\mathrm{Nd}^{3+}$ e $\mathrm{Cr}^{3+}$, utiliza-se um bombeio não ressonante ao nível laser 
emissor, em algum outro nível que apresente uma absorção maior, Seção 2.1. No entanto, com o surgimento dos lasers de diodo de alta potência em torno de $880 \mathrm{~nm}$ e dos cristais auto ativados (ou estequiométricos ${ }^{1}$ ), o bombeio ressonante ao nível laser emissor tornou-se uma alternativa viável e desejável, visto que ela diminui a geração de calor [1, 2, 17-20]. Por exemplo, o calor gerado bombeando o cristal NAB (dopado com 100\% de $\mathrm{Nd}^{3+}$ ) em $750 \mathrm{~nm}$ é $35 \%$ maior que o calor gerado em $882 \mathrm{~nm}$, da mesma forma bombeando em $808 \mathrm{~nm}$ gera 23\% mais calor que em $882 \mathrm{~nm}$ [2]. Em ambos os casos, excitação ressonante ou não ao nível laser emissor, é possível se modelar a influência do processo de upconversion Auger sobre os parâmetros do z-scan conhecendo o comportamento da população do estado excitado emissor. Neste capítulo mostraremos como a técnica de $z$-scan pode ser adaptada para obter o parâmetro de upconversion nas condições de excitação ressonante e não ressonante ao nível emissor, respectivamente.

\subsection{Excitação não ressonante ao nível emissor}

Iniciaremos nossa análise a partir do bombeio com excitação não ressonante ao nível laser emissor, onde não existe emissão estimulada, que é o mais comum esquema de bombeamento. Na Figura 5-2 vê-se um esquema de bombeamento não ressonante para o $\mathrm{Nd}^{3+}$ num cristal de YAG. Quando o processo de upconversion ocorre, mais um canal de decaimento não radiativo é adicionado à dinâmica do sistema laser. Neste caso torna-se necessário introduzir a taxa de decaimento por upconversion Auger que é dada por $W_{u p}=\gamma N_{e x}$, onde $\gamma$ é o parâmetro de upconversion $\left(\mathrm{cm}^{3} / \mathrm{s}\right)$ e $N_{e x}$ a concentração de população do nível laser superior $\left(\mathrm{cm}^{-3}\right)$.

\footnotetext{
${ }^{1}$ Este tipo de cristal recebe este nome porque o íon emissor entra diretamente na rede cristalina como um dos íons formadores.
} 


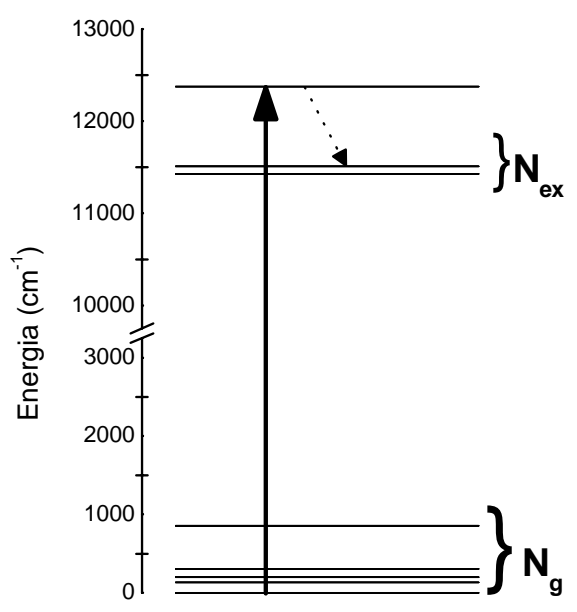

Figura 5-2 - Diagrama para excitação não ressonante ao nível emissor para uma amostra dopada com $\mathrm{Nd}^{3+}:$ YAG.

Assim, para obter $N_{e x}$ escrevemos a equação de taxa para um sistema da Figura 5-2 como:

$$
\frac{d N_{e x}}{d t}=\left(\frac{\sigma_{g} I}{h v}\right) N_{g}-\frac{N_{e x}}{\tau_{0}}-\gamma N_{e x}^{2}
$$

em que $\sigma_{g}$ é a seção de choque de absorção do estado fundamental para o excitado, $h$ é a constante de Planck, $v$ é a freqüência de excitação, $\tau_{0}$ o tempo de vida do nível emissor e $\gamma$ o parâmetro de upconversion Auger, que tem unidade de $\mathrm{cm}^{3} / \mathrm{s}$. Note que no regime de baixa intensidade de bombeamento $\gamma N_{e x}<<\tau^{-1}$ e o último termo da (5-1 torna-se desprezível. Lembrando que $S=I / I_{S}$ e $N_{t}=N_{e x}+N_{g}$ podemos re-escrever a Equação (5-1 como:

$$
\tau \frac{d N_{e x}}{d t}=S N_{t}-(S+1) N_{e x}-\gamma \tau_{0} N_{e x}^{2}
$$

que no regime estacionário $\left(t \rightarrow \infty\right.$ e $\left.d N_{e x} / d t=0\right)$ leva à solução:

$$
n_{e x}=\frac{N_{e x}}{N_{t}}=\frac{-(1+S)+\sqrt{(1+S)^{2}+4 \beta S}}{2 \beta}
$$

em que $\beta=\gamma N_{t} \tau$. A Figura 5-3 mostra curvas de $n_{e x}$ como função do parâmetro de saturação, $S$, para diferentes valores do $\beta$. Podemos ver que à medida que $\beta$ aumenta, diminui a população do estado 
excitado, para um mesmo valor de $S$, ou seja, o processo de upconversion introduz uma perda de população no nível laser. Note que tomando $\beta \rightarrow 0$ recupera-se a expressão para a população do estado excitado em um sistema de três níveis, $N_{e x}=N_{t} S /(1+S)$.

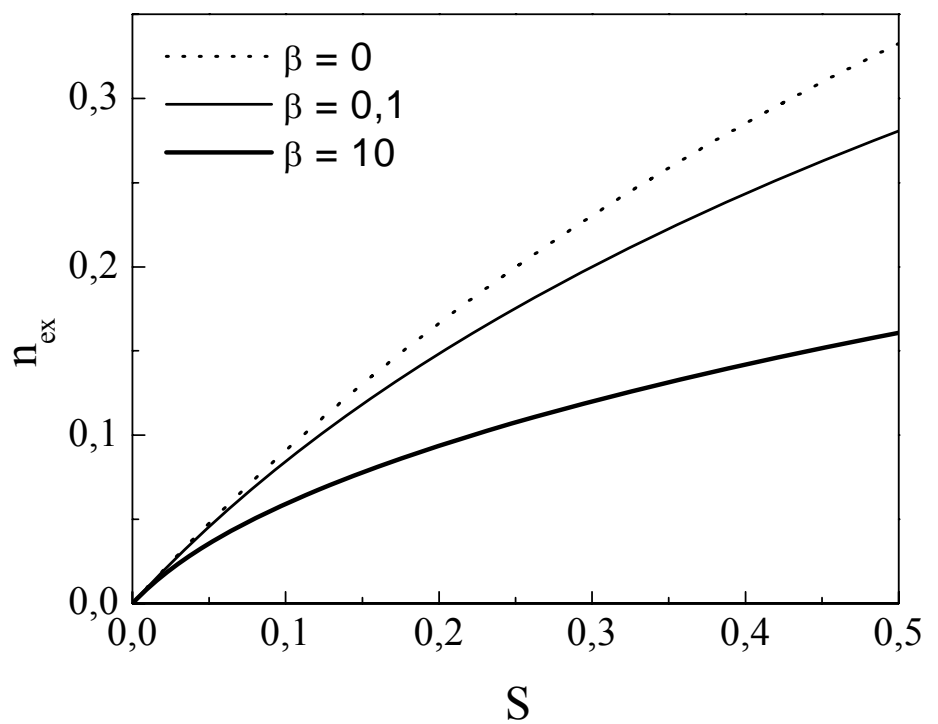

Figura 5-3 - Simulação da fração de população do estado excitado em função do parâmetro de saturação $S$, para vários valores do parâmetro de perda $\beta$ [21].

Um dos efeitos deste processo de transferência de energia sobre o sistema pode ser visto na intensidade de saturação. Normalmente, para um sistema de três níveis, $I_{S}$ é definida como a intensidade onde a população do estado excitado é metade da população do estado fundamental de maneira que a absorção linear cai para $1 / 2$ do seu valor não saturado. Assim, desconsiderando a influência do upconversion Auger (caso $\beta=0$ ), obteremos $N_{e x} / N_{t}=1 / 2$ para $I=I_{S}$. No entanto, isto não ocorre se nós tomamos $S=1$ na Equação (5-3. Mas fazendo $N_{e x} / N_{t}=1 / 2$ nesta equação nós podemos encontrar uma intensidade efetiva de saturação dada por:

$$
I_{S \beta}=I_{S}\left(1+\frac{\beta}{2}\right)
$$

Esta diferença pode ser explicada da seguinte forma: quando a transferência de energia ocorre entre os íons excitados, parte da população de $N_{e x}$ é transferida não radiativamente para o estado 
fundamental, Figura 5-1. Este decremento na população de $N_{e x}$ faz com que uma intensidade de bombeio maior deva ser utilizada para que se tenha uma população igual nos dois níveis. Assim, esta nova intensidade de saturação deve ser maior que $I_{S}$ por um fator de $1+\frac{\beta}{2}$. Novamente podemos notar que para $\beta=0$ recupera-se o valor conhecido da intensidade de saturação.

Normalmente, para este tipo de excitação, a técnica mais usada para obter o parâmetro $\beta$ é através do decaimento da fluorescência, que é proporcional à fração de população no nível excitado, $n_{e x}$ [10]. Após um pulso laser uma parte da população do estado fundamental é deslocada para o nível excitado. Nesta condição, é possível se modelar o decaimento da população tomando $S=0$ na Equação (5-2, de forma que $n_{e x}$ é agora dado por:

$$
n_{e x}(t)=\frac{n_{e x}(0) e^{-t / \tau}}{1+\beta n_{e x}(0)\left(1-e^{-t / \tau}\right)}
$$

onde $n_{e x}(0)$ é a fração de população do estado excitado para o tempo $\mathrm{t}=0$ (início do decaimento). $\mathrm{Na}$ Figura 5-4 vemos o comportamento transiente do decaimento da fluorescência para diferentes valores de $\beta$, obtida da Equação (5-5. Como pode ser visto desta figura, apenas para valores de $\mathrm{t} / \tau$ pequenos existe uma variação perceptível no decaimento. 


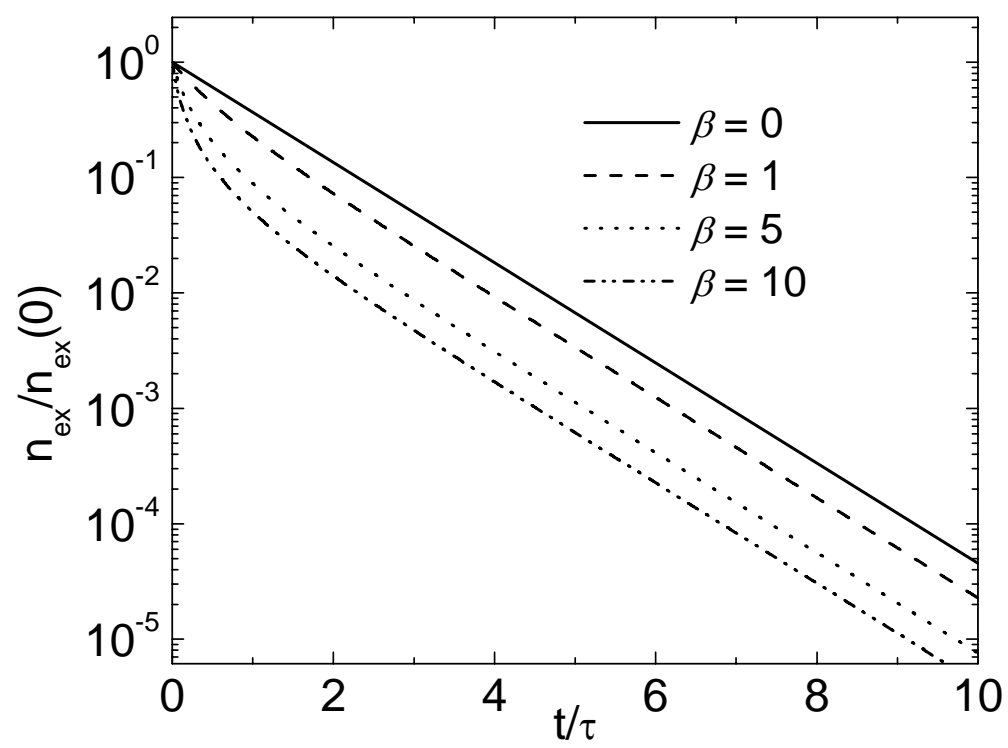

Figura 5-4 - Comportamento transiente da fluorescência do nível laser para diferentes valores do parâmetro $\beta$.

A principal desvantagem desta técnica é necessitar de potências muito altas, próxima ao limiar de dano do material [13], para obter uma curva onde o ajuste possibilite uma boa determinação de $\beta$.

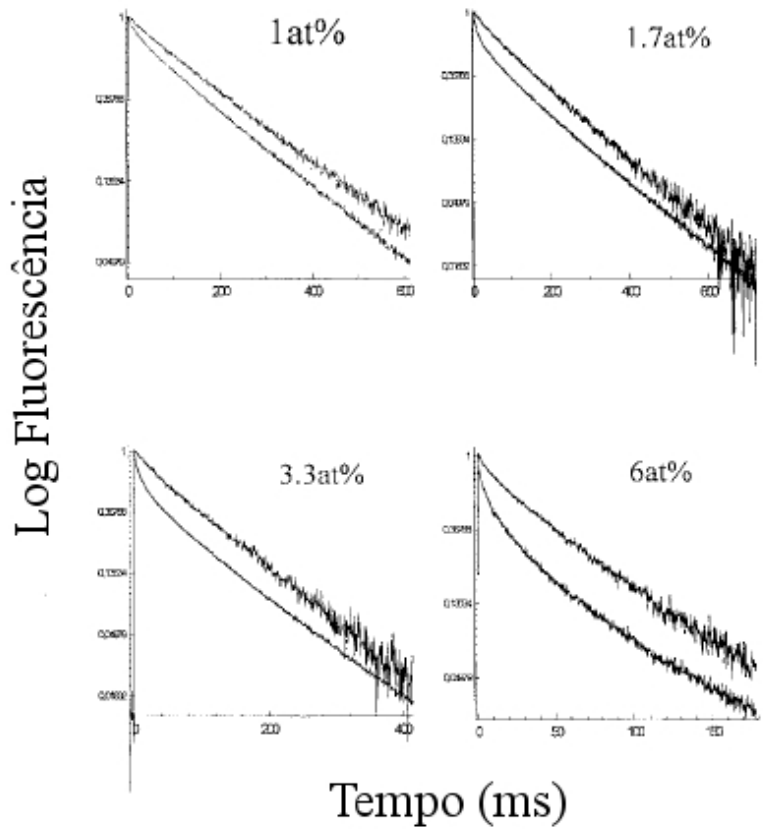

Figura 5-5 - Decaimentos obtidos para excitação baixa $\left(\mathrm{n}_{\mathrm{ex}}(0)<10^{-2}\right)$, curva mais alta, e para alta excitação $\left(\mathrm{n}_{\mathrm{ex}}(0) \sim 0,2\right.$ e 0,9$)$, curva mais baixa, em amostras de $\mathrm{Nd}^{3+}$ para diferentes concentrações [11]. 
A Figura 5-5 exibe os resultados encontrados por Guy et al [11] para cristais de $\mathrm{Nd}^{3+}: \mathrm{YAG}^{\mathrm{c}}$ com diferentes concentrações. Pode-se ver que o desvio do decaimento exponencial torna-se mais evidente para altas potências e altas concentrações (como esperado pois $W_{u \mathrm{p}} \propto N_{e x}$, que aumenta com a concentração). Note que os valores de $n_{e x}(0)$ podem chegar a $20 \%$, para os dois gráficos de cima, e 90\%, nos dois gráficos de baixo, o que é um bom indicativo das altas potências utilizadas.

Outra forma de obter os parâmetros relativos ao processo de upconversion Auger é utilizar a técnica de LT para medir o acréscimo do calor decorrente do processo (vide Figura 5-1). Esta metodologia foi introduzida por Pilla et al [15] para materiais dopados com $\mathrm{Cr}^{3+}$. Neste caso, lembrando que a taxa de decaimento total agora é dada por $W_{T}=\tau^{-1}+W_{u p}$, a eficiência quântica de fluorescência passa a ser escrita como $\eta=W_{R} / W_{T}$, ou seja:

$$
\eta=\frac{\eta_{0}}{1+\beta n_{e x}}
$$

onde $\eta_{0}$ é a eficiência quântica à baixa intensidade. Esta diminuição em $\eta$ provoca um aumento na quantidade de calor gerado, como pode ser visto na Equação 2-8. Usando as Equações 2-7, 2-8, 2-9 e 56, pode-se ajustar o comportamento do sinal de LT normalizado pela potência absorvida, $\Theta$, como função de $S$ e obter o parâmetro $\gamma$. 


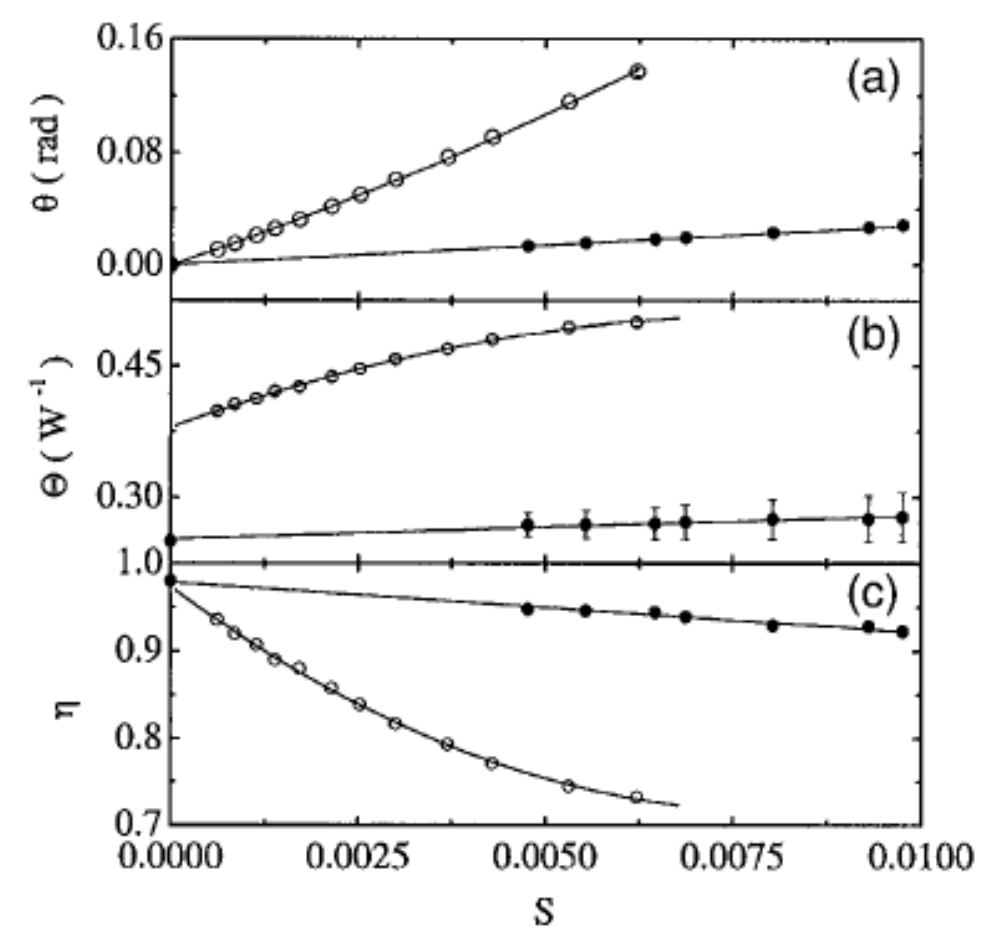

Figura 5-6 - (a) amplitude do sinal de LT; (b) $\theta$ normalizado pela potência absorvida e (c) comportamento da eficiência quântica como função de $\mathrm{S}$ para o cristla de $\mathrm{Cr}^{3+}$ :LiSAF com dopagens de $1 \%$ (círculos fechados) e 7\% (círculos abertos) [15].

Como pode ser visto na Figura 5-6 esta técnica requer muito menos potência que a anterior. Entretanto suas desvantagens ficam por conta do alinhamento e do fato de serem empregados dois lasers na medida. De fato, a determinação das cinturas dos feixes e suas posições relativas à amostra, assim como a sobreposição entre eles na amostra, são fatores críticos na determinação de $\gamma$.

Uma alternativa aos métodos anteriores é a determinação do parâmetro $\gamma$ através da técnica de $z$ scan.. Como visto no Capítulo 3 a redistribuição de população provocada pelo bombeamento muda algumas propriedades físicas do meio como a refração e a absorção. Como a técnica de $z$-scan é sensível a estas mudanças, então é possível utilizá-la para obter o parâmetro de upconversion. A idéia básica do método proposto é obter o parâmetro de upconversion através de uma medida de absorção não linear.

Uma vez calculada a população do estado excitado, Equação (5-3, podemos então escrever a absorção do material como sendo: 


$$
\begin{aligned}
\alpha_{N L} & =N_{g} \sigma_{g}+N_{e x} \sigma_{e x} \\
& =\alpha_{a b s}+\Delta \alpha_{a b s}
\end{aligned}
$$

onde usamos $N_{t}=N_{e x}+N_{g}$, com $\alpha_{a b s}=N_{t} \sigma_{\mathrm{g}}$ é o coeficiente de absorção linear, $\Delta \alpha_{a b s}=N_{e x} \Delta \sigma$ e $\Delta \sigma=\sigma_{e x}-$ $\sigma_{g}$ a diferença de seção de choque de absorção entre os estados excitado e fundamental. Para calcularmos a transmitância, $T$, usaremos a lei de Beer. ${ }^{2}$ Desta forma:

$$
T=\frac{I}{I_{0}}=e^{-\alpha_{N L s} L}=e^{-\left(\alpha_{a b s}+\Delta \alpha_{a b s}\right) L}
$$

mas, em geral $\Delta \alpha_{a b s} L<<1$. Sendo assim podemos fazer a expansão da exponencial e chegar ao resultado:

$$
\begin{aligned}
T & =e^{-\alpha_{a b s} L}\left(1-\Delta \sigma N_{e x} L\right) \\
& =T_{0}(1-\Delta T)
\end{aligned}
$$

em que $T_{0}=e^{-\alpha_{a b s} L}, \Delta T=\Delta \sigma N_{e x} L$ é a transmitância não linear. Usando agora o valor de $N_{e x}$ calculado anteriormente, (5-3, chegamos finalmente à expressão da transmitância não linear como função do parâmetro de upconversion Auger no regime estacionário:

$$
\Delta T=\Delta \sigma L N_{t}\left\{\frac{-(1+S)+\sqrt{(1+S)^{2}-4 \beta S}}{2 \beta}\right\}
$$

Entretanto, deve-se notar que esta equação depende da coordenada radial, ou seja, $\Delta T=$ $\Delta T(r)$ uma vez que $S=I(r) / I_{S}$, de modo que é necessário se integrar a equação na área iluminada da amostra. Infelizmente, esta integral não tem solução analítica, mas Andrade mostrou [21], usando simulações numéricas que é possível usar a Equação (5-10 substituindo $I(r)$ por sua média radial,

\footnotetext{
${ }^{2}$ À princípio a Lei de Beer se aplica apenas a partículas não interagentes. Como no nosso caso os íons interagem entre si, incluímos um termo de correção na absorção, $\Delta \alpha_{a b s}$, para levar em conta este fato.
} 
$<I>=I_{0} / 2,{ }^{3}$ até o limite de $S=1$, onde a diferença entre os resultados é de aproximadamente $8 \%$. Assim, para obter o parâmetro $S$, devemos considerar a intensidade média ao longo da amostra e da direção radial, tal que $\mathrm{S}=\langle\mathrm{I}\rangle / \mathrm{I}_{\mathrm{s}}$, sendo $\langle I\rangle=I_{0} L_{e f} / 2 L, \mathrm{I}_{0}=2 \mathrm{P} / \pi \mathrm{w}^{2}$. É importante lembrar que no cálculo da intensidade também devemos levar em conta a reflexão da amostra, $R$. De tal forma que teremos uma intensidade média dada por $\langle I\rangle=P(1-R) L_{e f} /\left(L \pi w^{2}\right)$. Experimentalmente esta variação da absorção não linear pode ser obtida da curva de $z$-scan com a íris totalmente aberta, onde seu valor máximo está na cintura do feixe de excitação $\left(\Delta T\right.$ (máx) $=T_{p}-1$, onde $T_{p}$ é a transmitância no pico). Para cada amostra fizemos diversas medidas de z-scan, com a fenda aberta, para várias potências incidentes e graficamos a máxima variação da transmitância como função da intensidade incidente. O parâmetro de upconversion, $\gamma$, foi então obtido a partir do ajuste com a Equação (5-10. Este mesmo procedimento foi adotado para o caso ressonante que será abordado a seguir. O valor de $\Delta \sigma$ pode ser obtido das medidas à baixa intensidade. Da Figura 5-7 até a Figura 5-9 vemos diversas medidas de variação da transmitância como função da intensidade incidente para várias amostras diferentes dopadas com $\mathrm{Nd}^{3+}$. O caso $\beta=0$ é mostrado para o cristal de YAG, para comparação. Já na Figura 5-10 temos uma amostra do cristal LiSAF dopada com $\mathrm{Cr}^{3+}$ que foi bombeada não ressonantemente em $488 \mathrm{~nm}$ na banda ${ }^{4} \mathrm{~T}_{1}$.

${ }^{3}$ Chamando $r / w=\rho$ podemos calcular a média radial como: $\langle I\rangle=\frac{\int_{0}^{\infty} I(\rho) e^{-2 \rho^{2}} 2 \pi \rho d \rho}{\int_{0}^{\infty} e^{-2 \rho^{2}} 2 \pi \rho d \rho}=I_{0} / 2$, onde $I(\rho)=I_{0} e^{-2 \rho^{2}}$. 


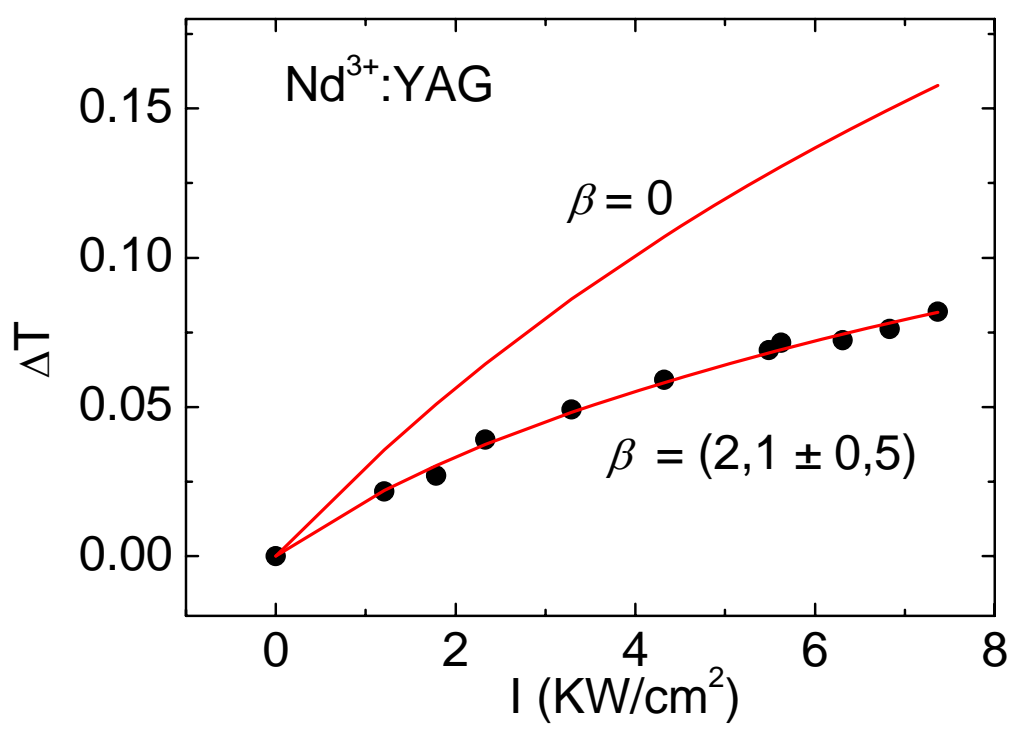

Figura 5-7 - Variação da transmitância para o cristal de YAG dopado com $\mathrm{Nd}^{3+}$. Usando $\lambda=808 \mathrm{~nm}, \Delta \sigma=2,8 \times 10^{-20} \mathrm{~cm}^{2}$, e $N_{t}=6,1 \times 10^{19} \mathrm{~cm}^{-3}$ foram obtidos $\beta=(2,1 \pm 0,5)$ e $I_{S}=(15 \pm 1) \mathrm{KW} / \mathrm{cm}^{2}$ usando a Equação (5-10 para o ajuste, linha cheia. $O$ caso $\beta=0$ também é mostrado para comparação.

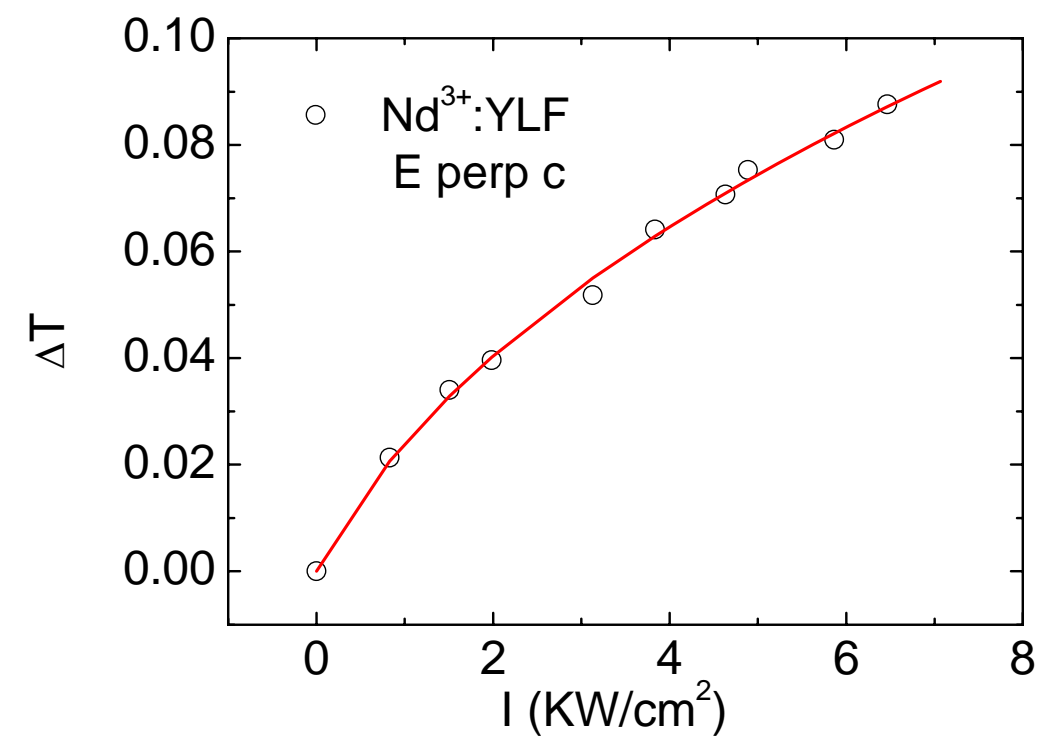

Figura 5-8 - Variação da transmitância para o cristal de YLF dopado com $\mathrm{Nd}^{3+}$. Usando $\lambda=792 \mathrm{~nm}$, foram obtidos $\beta=(11 \pm 2) e I_{S}=(25 \pm 2) \mathrm{KW} / \mathrm{cm}^{2}$. 


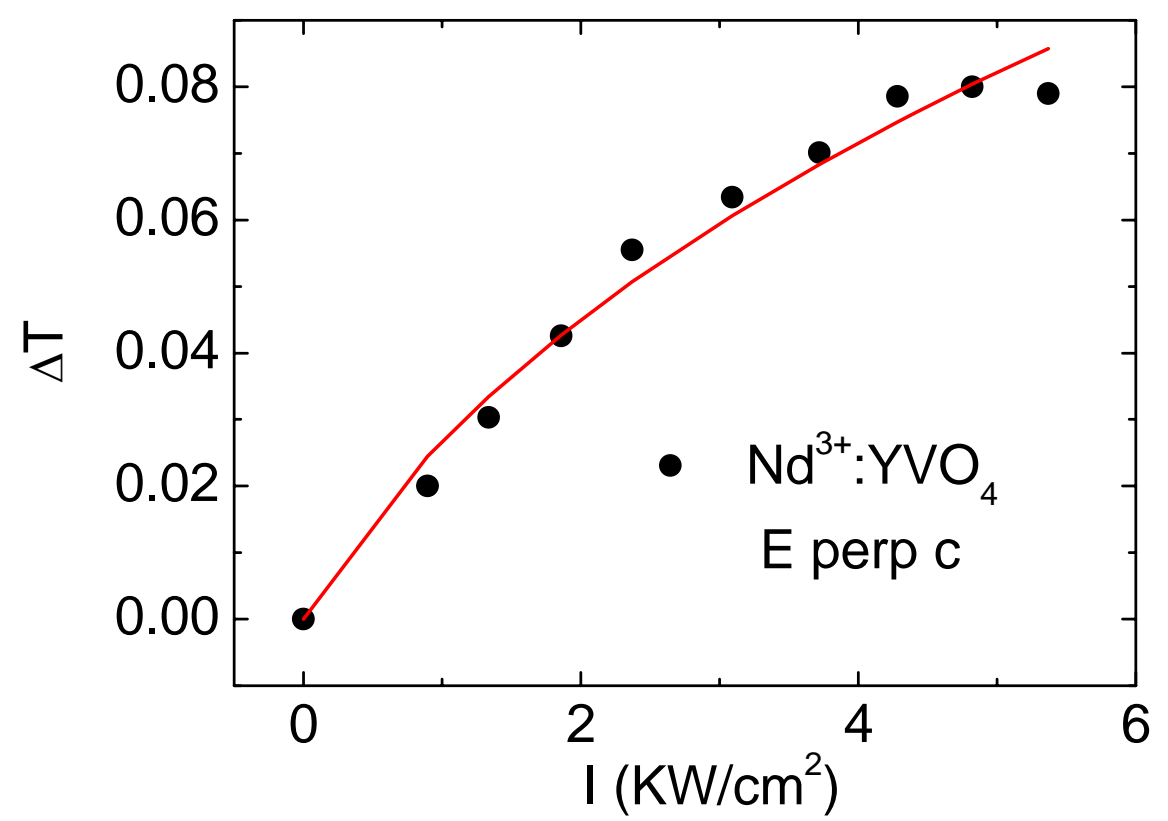

Figura 5-9 - Variação da transmitância para o cristal de $\mathrm{YVO}_{4}$ dopado com $\mathrm{Nd}^{3+}$. Usando $\lambda=808 \mathrm{~nm}$, foram obtidos $\beta=(4,6 \pm 2,3)$ e $I_{S}=(13 \pm 3) \mathrm{KW} / \mathrm{cm}^{2}$.

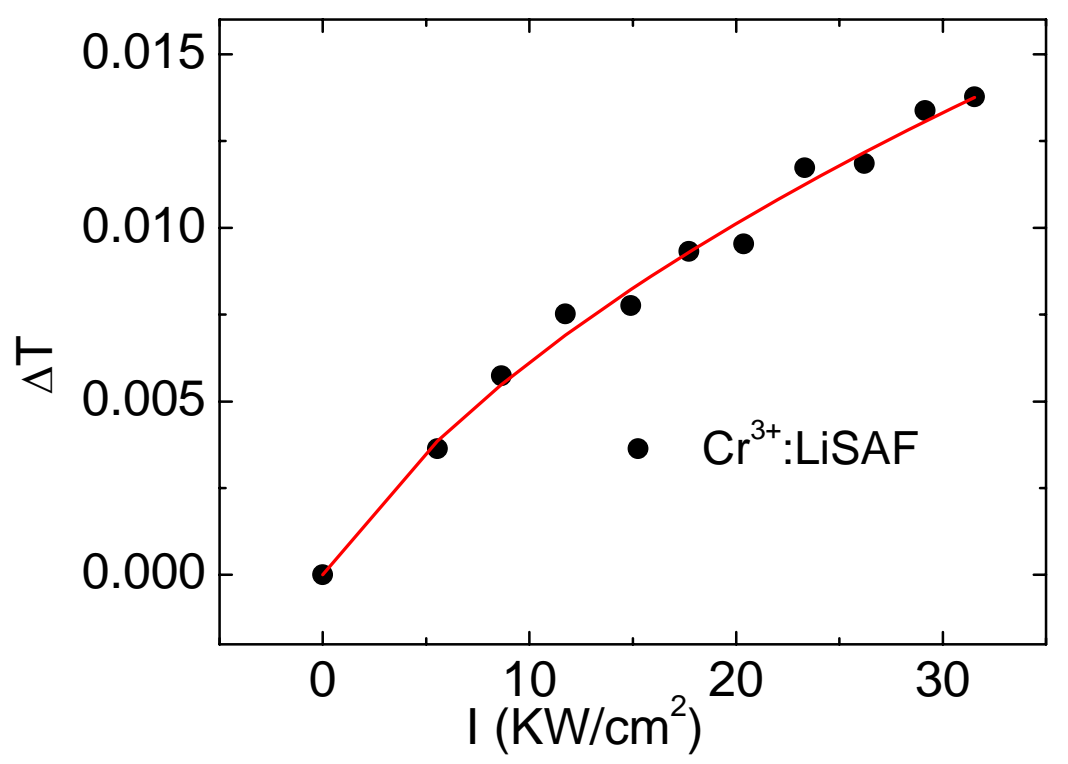

Figura 5-10 - Variação da transmitância para o cristal de LiSAF dopado com $\mathrm{Cr}^{3+}$. Usando $\lambda=808 \mathrm{~nm}$, foram obtidos $\beta=(61 \pm 18)$ e $I_{S}=(904 \pm 124) \mathrm{KW} / \mathrm{cm}^{2}$. 
Tanto os resultados obtidos como os presentes na literatura estão dispostos na Tabela 5-1 e na Tabela 5-2. Pode-se perceber uma boa concordância entre os valores calculados e os obtidos para a intensidade de saturação na Tabela 5-1, para os materiais estudados. Já os resultados do parâmetro de upconversion apresentam um certo espalhamento nos valores, quando comparados com os resultados da literatura. Este espalhamento deve-se principalmente aos diferentes métodos de medida empregados. Mas, dentro do erro das medidas, os valores que encontramos para $\gamma$ podem ser considerados de acordo com aqueles obtidos usando outras técnicas. Apesar disto pode-se notar, para um mesmo cristal, um aumento no valor de $\gamma$ com a concentração. Este aumento em $\gamma$ ocorre porque para maiores concentrações a distância entre os íons é menor, o que favorece a transferência de energia entre eles. Portanto, quanto maior a concentração de um dopante na matriz, mais calor relacionado com perdas por upconversion Auger ele gera.

Tabela 5-1 - Parâmetros usados nos ajustes: $\lambda(\mathrm{nm}), \tau(\mu \mathrm{s}), I_{S} *\left(\mathrm{KW} / \mathrm{cm}^{2}\right)$ calculado, $I_{S}$ $\left(\mathrm{KW} / \mathrm{cm}^{2}\right)$ obtido.

\begin{tabular}{ccccc}
\hline \hline Amostra & $\lambda$ & $\tau$ & $\boldsymbol{I}_{\boldsymbol{S}}{ }^{*}$ & $\boldsymbol{I}_{\boldsymbol{S}}$ \\
\hline \hline $\mathrm{Nd}^{3+}: \mathrm{YAG}$ & 808 & 250 & 14,1 & $15 \pm 1$ \\
$\mathrm{Nd}^{3+}: \mathrm{YLF}$ & 792 & 520 & 20,1 & $25 \pm 2$ \\
$\mathrm{E} \perp \mathrm{c}$ \\
$\begin{array}{c}\mathrm{Nd}^{3+}: \mathrm{YVO}_{4} \\
\mathrm{E} \perp \mathrm{c}\end{array}$ & 808 & 90 & 13,7 & $13 \pm 3$ \\
$\mathrm{Cr}^{3+}: \mathrm{LiSAF}(7 \%)$ & 488 & 65 & 964 & $904 \pm 124$ \\
$\mathrm{E} \| \mathrm{c}$ & & & & \\
\hline
\end{tabular}




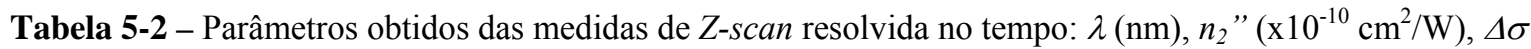
$\left(\mathrm{x} 10^{-20} \mathrm{~cm}^{2}\right), N_{t}\left(\times 10^{20} \mathrm{~cm}^{-3}\right), \gamma\left(\times 10^{-16} \mathrm{~cm}^{3} / \mathrm{s}\right)$

\begin{tabular}{|c|c|c|c|c|c|c|c|}
\hline Amostra & $\lambda$ & $n_{2} "$ & $\Delta \sigma$ & $N_{t}$ & $\gamma$ & Método & Ref. \\
\hline \multirow[t]{5}{*}{$\mathrm{Nd}^{3+}: \mathrm{YAG}$} & 808 & 7,8 & 2,8 & 0,61 & $1,4 \pm 0,6$ & z-scan & $\mathbf{a}$ \\
\hline & // & & & 1,03 & 2,4 & $z$-scan & [21] \\
\hline & // & & & 1,38 & 2,8 & fluorescência & [10] \\
\hline & // & & & 1,38 & 1,8 & // & [22] \\
\hline & // & & & 1,38 & 0,5 & // & [11] \\
\hline \multirow[t]{3}{*}{$\mathrm{Nd}^{3+}: \mathrm{YLF}$} & 792 & 9,5 & 1,8 & 1,7 & $1,2 \pm 0,2$ & z-scan & $\mathbf{a}$ \\
\hline & // & & & 1,7 & 1,9 & $\mathrm{LT}$ & [16] \\
\hline & // & & & 2,1 & 1,7 & fluorescência & [10] \\
\hline \multirow[t]{3}{*}{$\mathrm{Nd}^{3+}: \mathrm{YVO}_{4}$} & 808 & 15,1 & 5,2 & 0,62 & $8 \pm 4$ & z-scan & $\mathbf{a}$ \\
\hline & // & & & 0,75 & $20 \pm 10$ & numérico & [23] \\
\hline & $/ /$ & & & 0,75 & $15 \pm 5$ & fluorescência & [22] \\
\hline \multirow[t]{2}{*}{$\begin{array}{c}\mathrm{Cr}^{3+}: \text { LiSAF } \\
\text { E } \| \mathbf{c}\end{array}$} & 488 & 0,2 & 0,8 & 6,2 & $15 \pm 5$ & z-scan & $\mathbf{a}$ \\
\hline & $/ /$ & & & 6,2 & $20 \pm 4$ & $\mathrm{LT}$ & [15] \\
\hline
\end{tabular}

O significado físico do parâmetro de upconversion pode ser entendido definindo a população

crítica $\mathrm{N}_{\mathrm{cr}}$, dada por:

$$
N_{c r}=\left(\gamma \tau_{0}\right)^{-1}
$$

Assim, quando $N_{e x}=N_{c r}$ a taxa de upconversion será igual à taxa de decaimento por emissão espontânea, como pode ser visto na Equação (5-1. Desta forma foram então calculados $\mathrm{N}_{\mathrm{cr}}$ para os cristais listados acima como: $(2,8 \pm 1,2) \times 10^{19} \mathrm{~cm}^{-3},(1,6 \pm 0,3) \times 10^{19} \mathrm{~cm}^{-3},(1,3 \pm 0,7) \times 10^{19} \mathrm{~cm}^{-3},(1,0 \pm$ $0,3) \times 10^{19} \mathrm{~cm}^{-3}$ para $\mathrm{Nd}^{3+}: \mathrm{YAG} \mathrm{Nd}^{3+}: \mathrm{YFL} \mathrm{Nd}^{3+}: \mathrm{YVO}_{4}$ e $\mathrm{Cr}^{3+}:$ LiSAF, respectivamente. Logo, caso se queira então utilizar estes cristais como meio ativo para lasers que necessite armazenar energia (e. g., Q-switching), é necessário operar com população menor que $N_{c r}$ para que o sistema seja eficiente. 


\subsection{Excitação ressonante ao nível emissor}

Para o caso ressonante estudaremos apenas materiais dopados com $\mathrm{Nd}^{3+}$ pois, como dito no começo do capítulo, este tipo de excitação vem sendo progressivamente cada vez mais adotada. Aqui devemos considerar a emissão estimulada no comprimento de onda de bombeamento. Também se leva em conta as populações dos subníveis dos estados excitado e fundamental (pois a sintonização do laser de bombeio, Ti:Safira, nos permite excitar cada um dos subníveis por vez) para uma melhor descrição do processo.

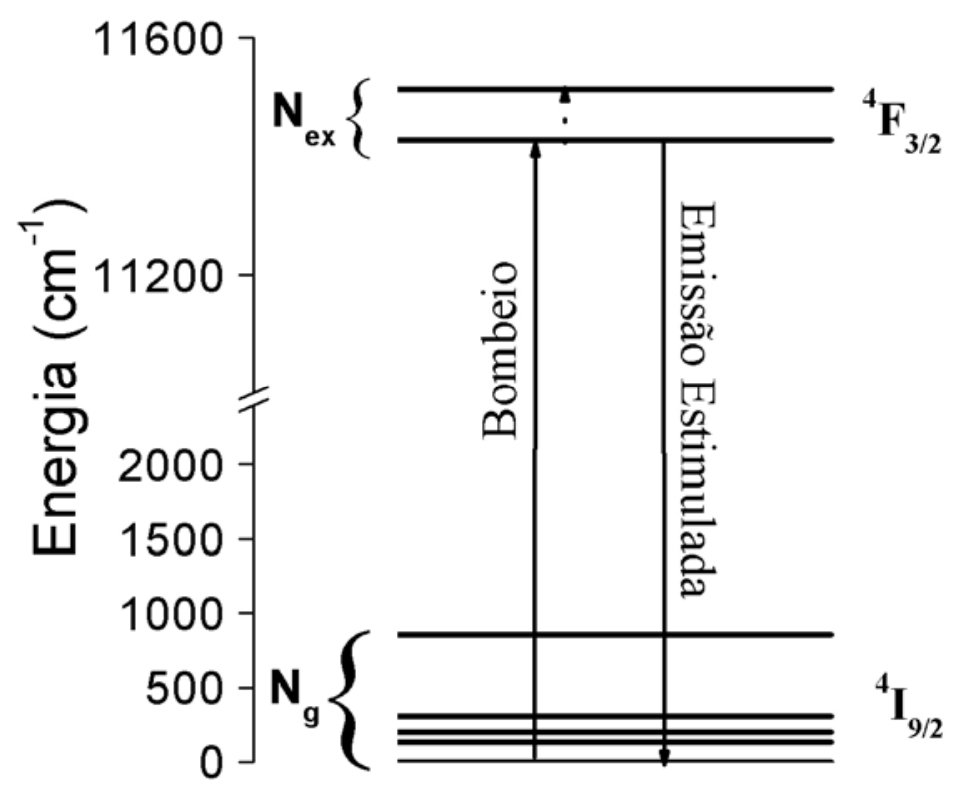

Figura 5-11 - Diagrama de níveis de energia do $\mathrm{Nd}^{3+}$ no cristal de YAG para o caso de excitação ressonante, mostrando seus níveis e subníveis de interesse.

Os subníveis do estado fundamental estão acoplados termicamente entre si devido à pequena separação em energia entre eles de modo que vale a distribuição de Boltzmann, ou seja:

$$
K_{i j}=\frac{N_{j}}{N_{i}}=e^{-\frac{\Delta E_{i j}}{k T}}
$$

onde $N_{i}$ é a população do nível $i$ e $\Delta E_{i j}$ a diferença de energia entre os níveis $j$ e $i, T$ é a temperatura da amostra, e $k$ aqui é a constante de Boltzmann. 
Assim, o estado fundamental é agora representado por seus subníveis, Figura 5-11, cujas populações denominaremos $N_{11}, N_{12}, N_{13}, N_{14}$ e $N_{15}$, de forma que $N_{g}=N_{11}+N_{12}+N_{13}+N_{14}+N_{15}$.

Portanto, podemos re-escrever as populações $N_{i j}$ da seguinte forma:

$$
\begin{aligned}
& N_{12}=N_{11} K_{12} \\
& N_{13}=N_{12} K_{23}=N_{11} K_{12} K_{23} \\
& N_{14}=N_{13} K_{34}=N_{11} K_{12} K_{23} K_{34} \\
& N_{15}=N_{14} K_{45}=N_{11} K_{12} K_{23} K_{34} K_{45}
\end{aligned}
$$

Logo a população total do nível $N_{g}$ se escreve como:

$$
\begin{aligned}
N_{g} & =N_{11}\left\{1+K_{12}+K_{12} K_{23}+K_{12} K_{23} K_{34}+K_{12} K_{23} K_{34} K_{45}\right\} \\
& =\frac{N_{11}}{f_{11}}
\end{aligned}
$$

onde $f_{11}=\left(1+K_{12}+K_{12} K_{23}+K_{12} K_{23} K_{34}+K_{12} K_{23} K_{34} K_{45}\right)^{-1}$ é a fração de população no subnível $N_{11}$.

Analogamente, o estado excitado será agora representado por seus dois subníveis cujas populações denominaremos $N_{21}$ e $N_{22}$, de forma que $N_{e x}=N_{21}+N_{22}$. Da mesma forma que no caso anterior, as populações destes subníveis estão termicamente acopladas, de modo que pode-se escrever:

$$
N_{e x}=\frac{N_{21}}{f_{21}}
$$

$\operatorname{com} f_{21}$ a fração de população no subnível $N_{21}$.

Devido a excitação ressonante consideraremos também um termo de emissão estimulada na equação de taxa que descreve o sistema, de forma que ela deve ser re-escrita como:

$$
\frac{d N_{e x}}{d t}=\left(\frac{\sigma_{12} I}{h v}\right) f_{11} N_{g}-\left(\frac{\sigma_{21} I}{h v}\right) f_{21} N_{e x}-\frac{N_{e x}}{\tau}-\gamma N_{e x}^{2}
$$


onde $\sigma_{12}$ é a seção de choque de absorção do nível fundamental para o excitado e $\sigma_{21}$ é a seção de choque de emissão do nível excitado para o fundamental. Fazendo a suposição que $\sigma_{21}=\sigma_{12}$, escrevendo agora $I_{S}$ como

$$
\begin{aligned}
I_{S} & =\frac{h v}{2\left(f_{11} \sigma_{12}\right) \tau} \\
& =\frac{h v_{12}}{2 \sigma \tau}
\end{aligned}
$$

onde $\sigma=f_{11} \sigma_{12}$ é a seção de choque de absorção do primeiro subnível do estado fundamental. Note que na (5-17, a intensidade de saturação é agora a de um sistema de dois níveis, devido ao bombeamento ressonante. Resolvendo a (5-16 para o caso estacionário, teremos:

$$
N_{e x}=\frac{-\left[\left(1+f_{21} / f_{11}\right) S+2\right]+\sqrt{\left[\left(1+f_{21} / f_{11}\right) S+2\right]^{2}+8 \beta S}}{4 \beta} N_{t}
$$

De acordo com esta equação a população do estado excitado depende agora da razão entre as frações de população nos subníveis mais baixos de cada nível, ou seja, $N_{e x}$ passa a depender também da temperatura do meio. Como no caso anterior, escreveremos agora a absorção efetiva do material em função dos parâmetros acima, de forma que:

$$
\begin{aligned}
\alpha_{N L} & =N_{g} \sigma+N_{e x} \sigma_{e x}-\sigma_{21} f_{22} N_{e x} \\
& =N_{t} \sigma+N_{e x}\left(\sigma_{e x}-\sigma-\sigma_{21} f_{22}\right) \\
& =\alpha_{a b s}+N_{e x} \Delta \sigma
\end{aligned}
$$

Daí, usando agora a lei de Beer chegamos à expressão da transmitância normalizada para a fenda aberta de forma análoga a Equação (5-10. De novo $\Delta T=\Delta \sigma L N_{e x}$, mas com $N_{e x}$ dado na Equação (5-18 e $\Delta \sigma$ considerando o efeito de emissão estimulada, Equação (5-19. 
As figuras abaixo apresentam resultados de medidas do parâmetro de upconvertion Auger para os mesmos materiais da seção anterior, à exceção do $\mathrm{Cr}^{3+}$ :LiSAF, usando agora a excitação ressonante. As frações das populações dos estados excitado e fundamental foram calculadas a partir de dados da literatura. São elas: para $\mathrm{Nd}^{3+}: \mathrm{YAG}_{11}=0,462$ e $f_{21}=0,6[12]$, para $\mathrm{Nd}^{3+}: \mathrm{YLF}_{11}=0,429$ e $f_{21}=0,57$ [24] e para $\mathrm{Nd}^{3+}: \mathrm{YVO}_{4} f_{11}=0,4$ e $f_{21}=0,52$ [24]. Com estes parâmetros e $\Delta \sigma$ medido à baixa intensidade foram obtidos $\gamma$ e $\mathrm{I}_{\mathrm{S}}$, como mostrado abaixo.

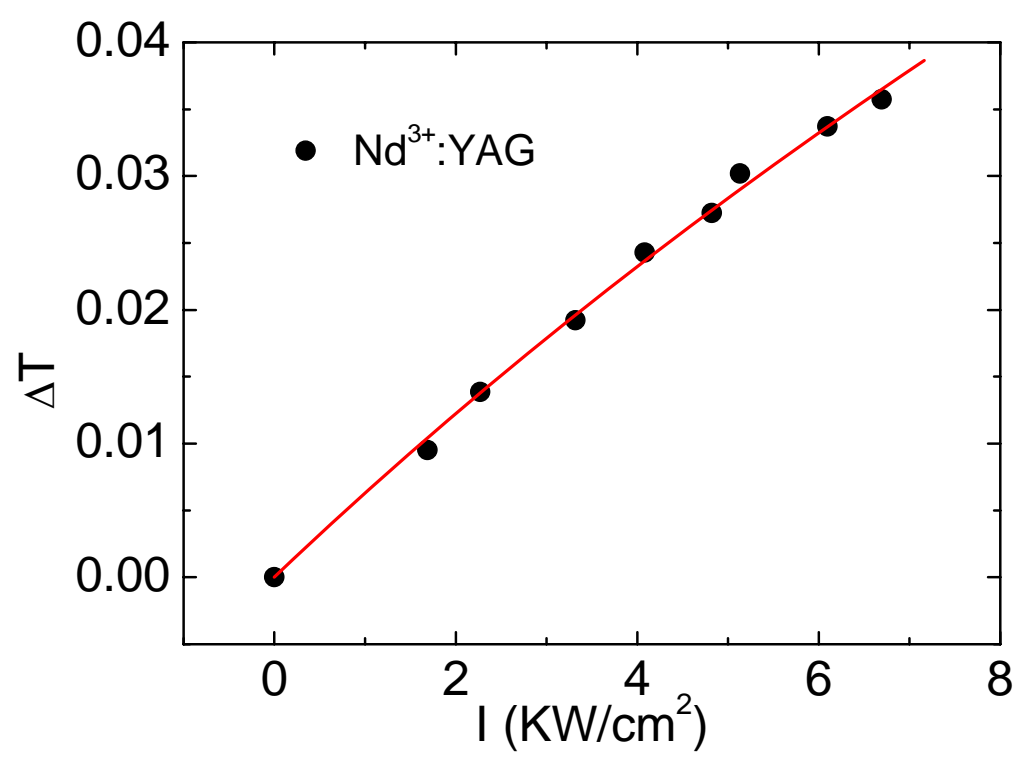

Figura 5-12 - Variação da transmitância num cristal de YAG dopado com $\mathrm{Nd}^{3+}$ sob excitação ressonante. No ajuste foram usados $\lambda=869 \mathrm{~nm}, f_{21}=0.6$ e $f_{11}=0.462 \mathrm{e}$ foram obtidos $\beta=(3 \pm 2)$ e $I_{S}=(87 \pm 4) \mathrm{KW} / \mathrm{cm}^{2}$. A linha cheia é o ajuste feito com a expressão para $\Delta T$ usando agora a Equação 5-18 para $N_{e x}$. 


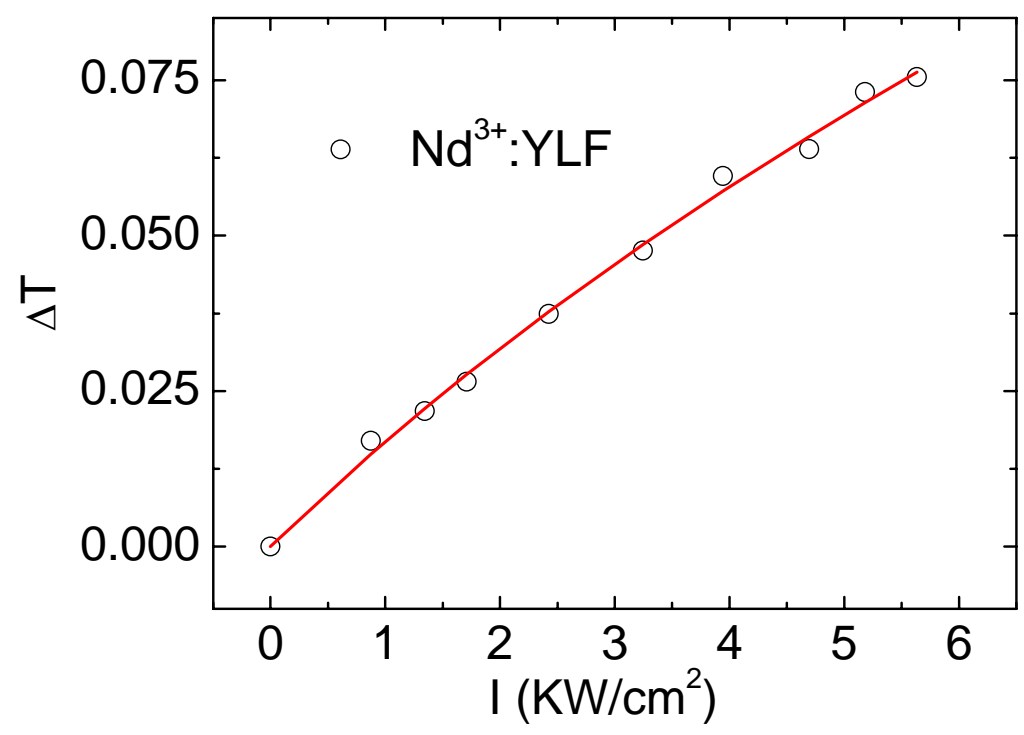

Figura 5-13 - Variação da transmitância num cristal de YLF dopado com $\mathrm{Nd}^{3+}$ sob excitação ressonante. No ajuste foram usados $\lambda=862 \mathrm{~nm} f_{21}=0,57$ e $f_{11}=0,429$ e foram obtidos $\beta=(7,5 \pm 3)$ e $I_{S}=(67 \pm 4) \mathrm{KW} / \mathrm{cm}^{2}$.

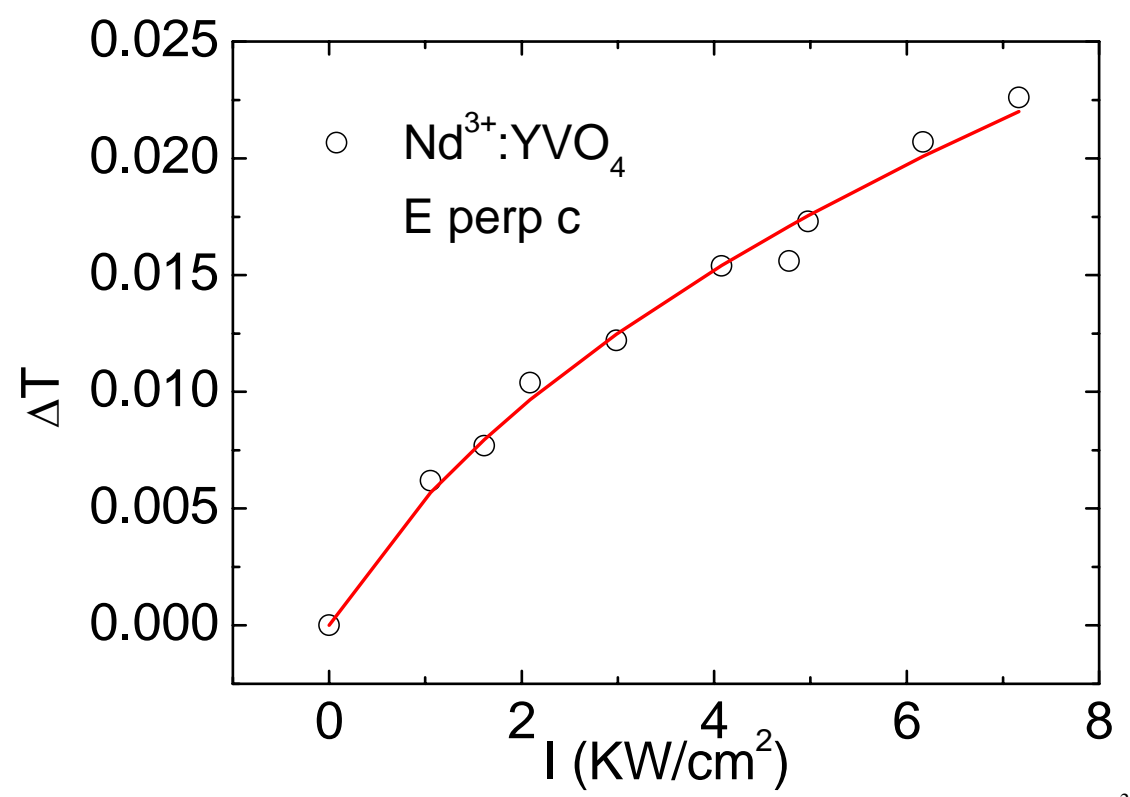

Figura 5-14 - Variação da transmitância num cristal de $\mathrm{YVO}_{4}$ dopado com $\mathrm{Nd}^{3+}$ sob excitação ressonante. No ajuste foram usados $\lambda=879 \mathrm{~nm} f_{21}=0,52 \mathrm{e} f_{11}=0,4$ e foram obtidos $\beta=(14 \pm 5)$ e $I_{S}=(24 \pm 3) \mathrm{KW} / \mathrm{cm}^{2}$.

Assim como no caso anterior as intensidades de saturação obtidas do ajuste estão em bom acordo com aquelas calculadas, como pode ser visto na Tabela 5-3. É interessante notar que existem poucos resultados na literatura para excitação ressonante. Por este motivo é difícil comparar nossos 
resultados com outros realizados utilizando outras técnicas. No entanto, para o YLF e $\mathrm{YVO}_{4}$ foi possível fazer esta comparação, como mostrado na Tabela 5-4. Pode-se ver que há uma boa concordância entre estes dados, o que ajuda a enfatizar a confiabilidade da técnica de $z$-scan na obtenção do parâmetro $\gamma$.

Tabela 5-3 - Parâmetros usados nos ajustes: $\lambda(\mathrm{nm}), \tau(\mu \mathrm{s}), I_{S} *\left(\mathrm{KW} / \mathrm{cm}^{2}\right)$ calculado, $I_{S}$ $\left(\mathrm{KW} / \mathrm{cm}^{2}\right)$ obtido.

\begin{tabular}{ccccc}
\hline \hline Amostra & $\boldsymbol{\lambda}$ & $\boldsymbol{\tau}$ & $\boldsymbol{I}_{\boldsymbol{S}}{ }^{*}$ & $\boldsymbol{I}_{\boldsymbol{S}}$ \\
\hline $\mathrm{Nd}^{3+}: \mathrm{YAG}$ & 869 & 250 & 92,3 & $87 \pm 4$ \\
$\mathrm{Nd}^{3+}: \mathrm{YLF}$ & 862 & 520 & 55,4 & $67 \pm 4$ \\
$\begin{array}{c}\mathrm{E} \perp \mathrm{c} \\
\mathrm{Nd}^{3+}: \mathrm{YVO}_{4}\end{array}$ & 879 & 90 & 27,8 & $24 \pm 3$ \\
$\mathrm{E} \perp \mathrm{c}$ & & & & \\
\hline
\end{tabular}

Tabela 5-4 - Parâmetros obtidos das medidas de Z-scan resolvida no tempo: $\lambda(\mathrm{nm}), n_{2}{ }^{\prime \prime}\left(\mathrm{x}^{-10} 0^{-10} \mathrm{~cm}^{2} / \mathrm{W}\right), \Delta \sigma$ $\left(\times 10^{-20} \mathrm{~cm}^{2}\right), N_{t}\left(\times 10^{20} \mathrm{~cm}^{-3}\right), \gamma\left(\times 10^{-16} \mathrm{~cm}^{3} / \mathrm{s}\right), N_{c r}\left(\times 10^{19} \mathrm{~cm}^{-3}\right)$

\begin{tabular}{|c|c|c|c|c|c|c|c|}
\hline Amostra & $\lambda$ & $n_{2} "$ & $\Delta \sigma$ & $\overline{N_{t}}$ & $\gamma$ & Método & Ref. \\
\hline \multirow{2}{*}{$\begin{array}{l}\mathrm{Nd}^{3+}: \mathrm{YAG} \\
\mathrm{Nd}^{3+}: \mathrm{YLF}\end{array}$} & 869 & 1,9 & 8,3 & 0,61 & $2,1 \pm 1,3$ & $z$-scan & $\mathrm{a}$ \\
\hline & 863 & 6,8 & 6,5 & 1,7 & $0,8 \pm 0,3$ & $z$-scan & $\mathrm{a}$ \\
\hline $\mathrm{E} \perp \mathrm{c}$ & // & & & 1,64 & $0,98 \pm 0,03$ & fluorescência & {$[25]$} \\
\hline \multirow[t]{2}{*}{$\begin{array}{c}\mathrm{Nd}^{3+}: \mathrm{YVO}_{4} \\
\mathrm{E} \perp \mathrm{c}\end{array}$} & 879 & 15,1 & 5,2 & 0,62 & $25 \pm 8$ & $z$-scan & $\mathrm{a}$ \\
\hline & 1060 & & & 1,5 & $30 \pm 10$ & $\begin{array}{c}\text { Oscilação de } \\
\text { potência }\end{array}$ & [14] \\
\hline
\end{tabular}

Uma vez que o processo de upconversion acontece sempre do mesmo nível, ${ }^{4} \mathrm{~F}_{3 / 2}$, é de se esperar que para o bombeio ressonante ou Stokes (acima do nível emissor) o resultado para $\gamma$ não seja muito diferente. De fato, comparando a Tabela 5-4 à Tabela 5-2 vemos que, dentro do erro da medida, os resultados encontrados concordam, exceto para o cristal de $\mathrm{YVO}_{4}$ que apresenta um erro de $\sim 50 \%$ na medida não ressonante. Este erro pode ser diminuído melhorando-se a sensibilidade na detecção do sinal, pois a imposição de se usar a intensidade média ao invés de $I(r)$ nas Equações 4-8 e 4-17 nos 
impossibilita de usar maiores potências no intuito de aumentar a razão sinal/ruído de experimento. As densidades críticas de população nestes comprimentos de onda foram $(1,9 \pm 1,1) \times 10^{19} \mathrm{~cm}^{3},(2,3 \pm$ $0,8) \times 10^{19} \mathrm{~cm}^{3}$ e $(0,4 \pm 0,1) \times 10^{19} \mathrm{~cm}^{3}$, para os cristais de $\mathrm{Nd}^{3+}: \mathrm{YAG}, \mathrm{Nd}^{3+}: \mathrm{YLF}$ e $\mathrm{Nd}^{3+}: \mathrm{YVO}_{4}$, respectivamente. Mais uma vez, os valores concordam dentro da margem de erro da técnica.

Como visto nas seções acima a técnica de z-scan apresenta diversas vantagens quando comparadas às técnicas usualmente empregadas para a determinação do parâmetro de upconversion $\gamma$. Entre estas vantagens podemos destacar as baixas intensidades usadas, normalmente $\mathrm{S}<1$, o fácil alinhamento, pois no experimento toda a luz é focalizada no detector o que relaxa as condições de alinhamento, e o fato de usar uma única fonte laser em contraposição às duas necessárias na técnica de LT. Além disso, esta técnica é insensível à variação do índice de refração provocado pelo aquecimento da amostra, ao contrário da LT que para alguns materiais pode misturar o efeito térmico com o eletrônico.

Além de obter o parâmetro de upconversion via medidas com a fenda aberta, buscamos também caracterizar o efeito deste processo sobre a fenda fechada. Este trabalho será apresentado na seção seguinte.

\subsection{Efeito do upconversion Auger sobre a refração não linear}

Para obter o comportamento da transmitância devido à refração não linear para o caso de excitação não ressonante, faz-se primeiro necessário calcular a variação de fase induzida no feixe quando este atravessa a amostra. Esta variação induzida de fase pode ser escrita da seguinte forma:

$$
\Delta \phi=\frac{2 \pi}{\lambda} L_{e f} \Delta n
$$

No entanto, como visto no Capítulo 3 podemos escrever: 


$$
\Delta n=n_{2}^{\prime} I_{S} n_{e x}
$$

Esta expressão é válida considerando ou não o efeito de upconversion Auger. Logo, usando a Equação (5-3 podemos re-escrever a fase induzida como:

$$
\Delta \phi=\frac{2 \pi}{\lambda} L_{e f} n_{2}^{\prime} I_{S} \frac{\sqrt{(1+S)^{2}+4 \beta S}-(1+S)}{2 \beta}
$$

Procedendo de maneira análoga ao que foi apresentado no Capítulo 3, consideramos o campo elétrico na saída da amostra como sendo $E_{s}=E_{0} e^{-i \Delta \phi}$ e aplicamos então a integral de difração de Kirchhoff para obtermos a intensidade do feixe no campo distante, Apêndice B. A partir da intensidade pudemos, finalmente, calcular a transmitância como função da posição da amostra em relação à cintura do feixe incidente. Da mesma forma que para a absorção não linear, não nos foi possível encontrar uma solução analítica para esta integral, pois $\Delta \phi=\Delta \phi(\mathrm{r})$. na verdade, mesmo usando a aproximação $<\mathrm{I}>=\mathrm{I}_{0} / 2$ não é possível obter uma solução analítica da integral de difração, ficando disponível assim apenas a solução numérica. Ressaltamos que as simulações foram feitas levando em conta apenas o valor da intensidade no centro do feixe, $i$. e., não consideramos o efeito da abertura na integral de difração para efeito de simplificação da simulação.

Estas simulações foram feitas considerando $n_{2}{ }^{\prime}=5.6 \times 10^{-9} \mathrm{~cm}^{2} / \mathrm{W}$ e $I_{S}=18 \mathrm{KW} / \mathrm{cm}^{2}$, em $808 \mathrm{~nm}$, obtidos por Andrade para uma amostra de $\mathrm{Nd}^{3+}$ :YAG com uma dopagem de $1,03 \times 10^{20}$ íons $/ \mathrm{cm}^{3}$ [21]. Usando estes valores realizamos várias simulações numéricas no intuito de obter curvas de z-scan para a fenda fechada em diferentes potências. Destas curvas pudemos então graficar a variação da transmitância entre o pico e o vale, $\Delta T_{p v}$, como função da intensidade no foco e assim obter o melhor ajuste para os dados experimentais variando apenas o parâmetro $\beta$. A Figura 5-15 mostra o comportamento de $\Delta T_{p v}$, experimental e numérico, como função da intensidade incidente. Para o caso de $\Delta \phi$ não saturado sabemos que $\Delta T_{p v} \propto \Delta \phi \propto N_{e x}$. Assim, podemos esperar que esta mesma relação 
se mantenha para o caso onde o processo de upconversion Auger está presente. Logo, da mesma forma que no caso da fenda aberta o aumento do parâmetro $\beta$ leva a uma diminuição de $\Delta T_{p v}$ pois $N_{e x}$ decresce com $\beta$, Equação (5-3. Ainda não nos foi possível obter uma expressão analítica que relacione $\Delta T_{p v}$ com $\Delta \phi$ (como no caso do z-scan convencional). Mas para os resultados apresentados na Figura 5-15, conseguimos um bom ajuste para $\beta=6$, o que está em excelente acordo com $\beta=(6,3 \pm$ 0,8) obtido por Andrade para a fenda aberta [21]. Embora não seja o resultado de um ajuste real dos pontos experimentais, este valor indica que o tratamento empregado para analisar a transmitância da fenda fechada está correto e descreve bem o comportamento da refração não linear sob o efeito de upconversion Auger.

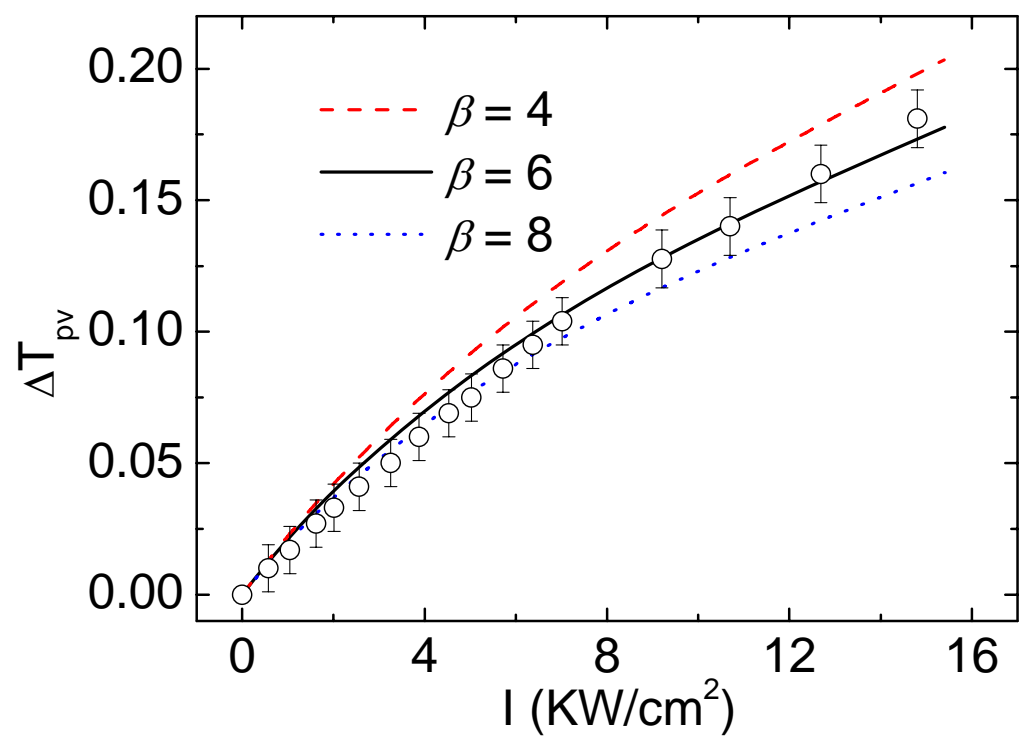

Figura 5-15 - Variação na transmitância entre pico e vale da curva de $z$-scan com a fenda fechada. Os círculos com barras de erro denotam os pontos experimentais, as demais curvas foram obtidas através de simulações numéricas. Usando $\boldsymbol{L}_{\text {ef }}=0.1 \mathrm{~cm}, n_{2}{ }^{\prime}=5,6 \times 10^{-9} \mathrm{~cm}^{2} / \mathrm{W}$ e $I_{s}=18 \mathrm{KW} / \mathrm{cm}^{2}$ obtivemos a melhor concordância para $\beta=6$. 


\subsection{Dinâmica da população do estado excitado sob o efeito de upconversion Auger}

Para entendermos a dinâmica completa de um sistema laser bombeado não ressonantemente, é necessário calcular também o comportamento transiente da população para a fase de excitação, i. e., $S \neq$ 0. Isto pode ser feito resolvendo então a Equação (5-2 sem as aproximações de $S=0$ (que remete ao decaimento da população) e $t \rightarrow \infty$ (que leva à resposta estacionária), de modo a chegarmos no resultado geral:

$$
n_{e x}=\frac{\sqrt{(1+S)^{2}+4 \beta S}+(1+S)+\left\{\sqrt{(1+S)^{2}+4 \beta S}-(1+S)\right\} \delta \exp t / \tau}{2 \beta(\delta \exp t / \tau-1)}
$$

onde

$$
\delta=\frac{2 \beta n_{e x}(0)+(1+S)+\sqrt{(1+S)^{2}+4 \beta S}}{2 \beta n_{e x}(0)+(1+S)-\sqrt{(1+S)^{2}+4 \beta S}}
$$

E o tempo característico da não linearidade é agora dependente de $\beta$ através de:

$$
\tau^{-1}=\tau_{0}^{-1} \sqrt{(1+S)^{2}+4 \beta S}
$$

A Figura 5-16 mostra o comportamento transiente de $n_{e x}$ tanto para $\beta$ fixo com $S$ variando como para $S$ fixo e $\beta$ variando. Desta figura nota-se que $n_{e x}$ satura mais rápido para maiores valores de $S$ e de $\beta$, diminuindo assim o tempo característico da não linearidade e, mostrando como estes fatores influenciam a fase em que o nível laser está sendo populado. 


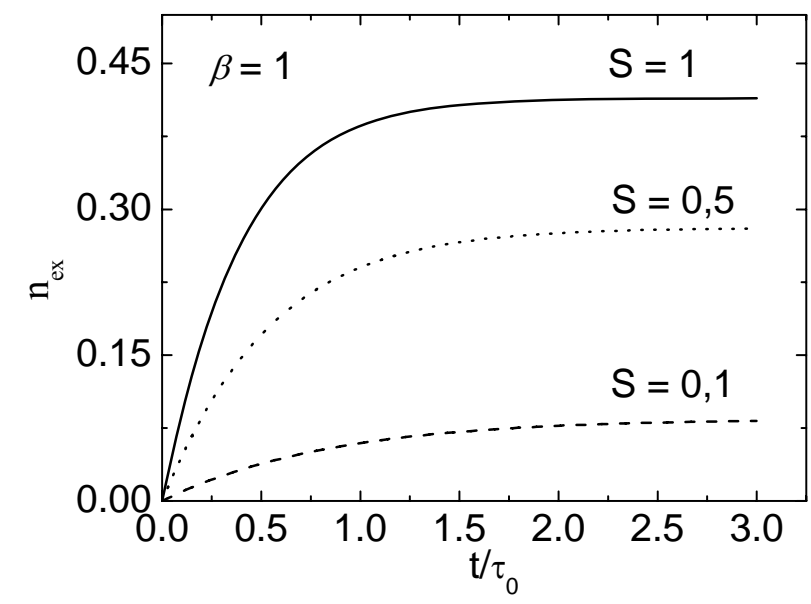

(a)

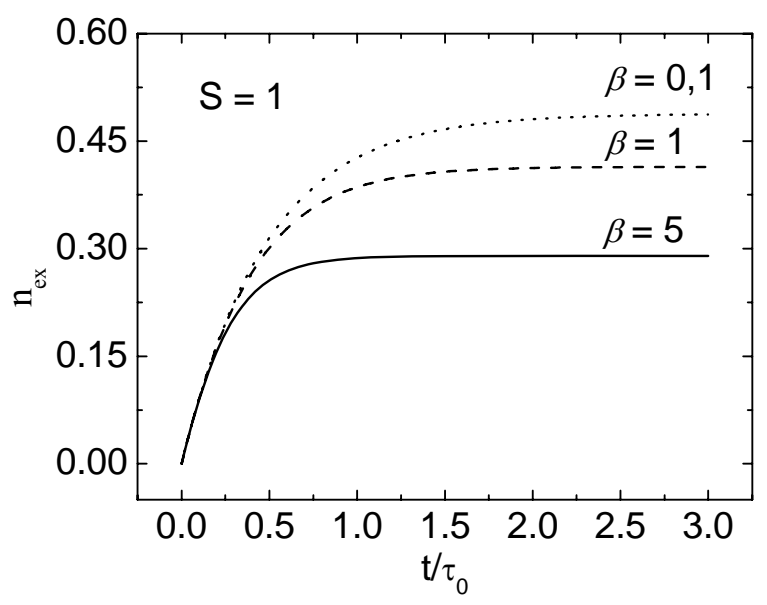

(b)

Figura 5-16 - Comportamento da fração de população do estado excitado para (a) valores diferentes de $\beta$ fixo e valores diferentes de $S$ e (b) $S$ fixo e valores diferentes de $\beta$.

Para $\mathrm{t} \rightarrow \infty$ é fácil notar que a Equação (5-23 retorna à forma anteriormente calculada para $\mathrm{o}$ estado estacionário, Equação (5-3. Da mesma maneira se tomarmos $S=0$ obteremos a Equação (5-5. Além disto, para o tempo característico da não linearidade, $\tau$, se tomarmos na Equação $(5-25 \beta=0$ obteremos a Equação 4-5 que representa $\tau$ para um sistema de três níveis sem efeitos de transferência de energia. Portanto, podemos dizer que as Equações 5-23 e 5-25 são generalizações das expressões para a população e o tempo de vida de um sistema de três níveis sob a influência do processo de upconversion Auger.

Assim, podemos então calcular o comportamento transiente do sinal transmitido para o caso da fenda aberta. A Figura 5-17 mostra o resultado experimental deste comportamento temporal para um cristal de $\mathrm{Nd}^{3+}: \mathrm{YAG}$, com a amostra fixa na posição de pico da curva de $z$-scan. 


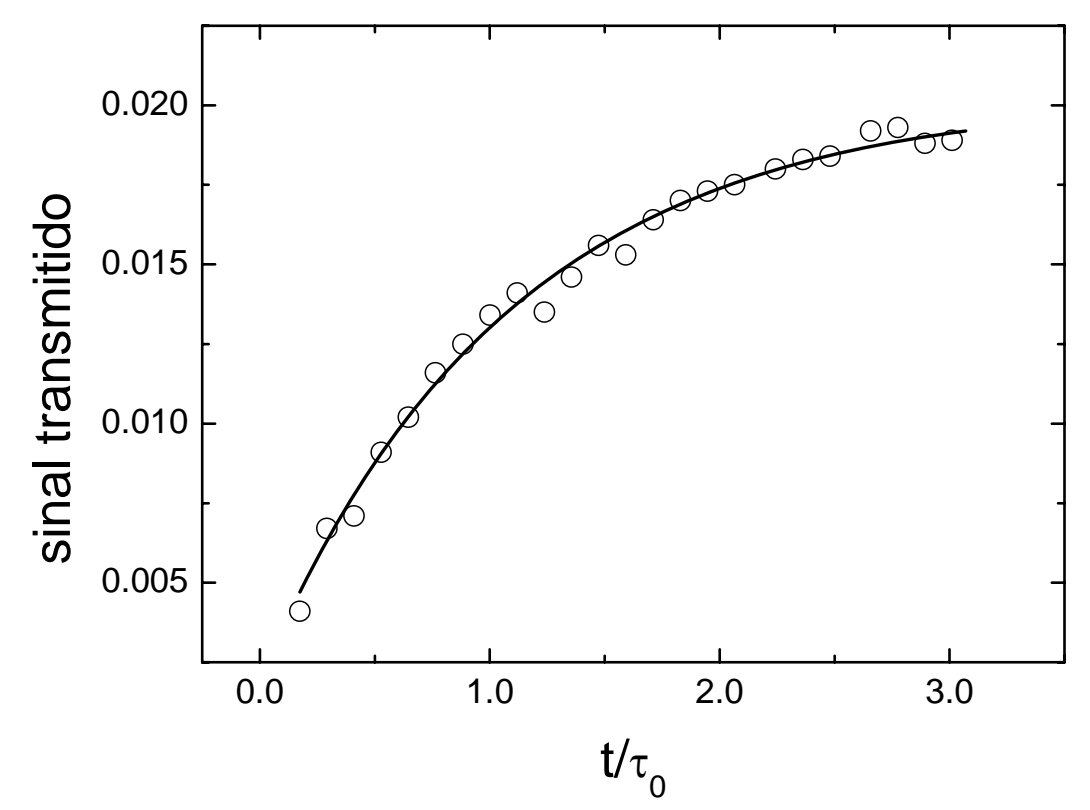

Figura 5-17 - Comportamento transiente do sinal transmitido da fenda aberta ajustado para um cristal de $\mathrm{Nd}^{3+}$ :YAG bombeado em $808 \mathrm{~nm}$ e uma concentração de $0,75 \%$, com as Equações (5-23 e 5-24. Neste ajuste $\tau_{0}=240$ $\mu$ s e $\mathrm{S}=0,5$.

Experimentalmente é possível a partir do ajuste desta curva, com a Equação (5-23, obter o valor de $\tau$ para cada intensidade incidente, graficar $\tau^{-1} x S$ e ajustar a curva com a Equação (5-25 para se obter os valores de $\tau_{0}$, e $\beta$, usando um tratamento análogo ao que foi empregado na Figura 3-6. A Figura 5-18 mostra curvas teóricas de $\tau^{-1} x S$ para diferentes valores de $\beta$. 


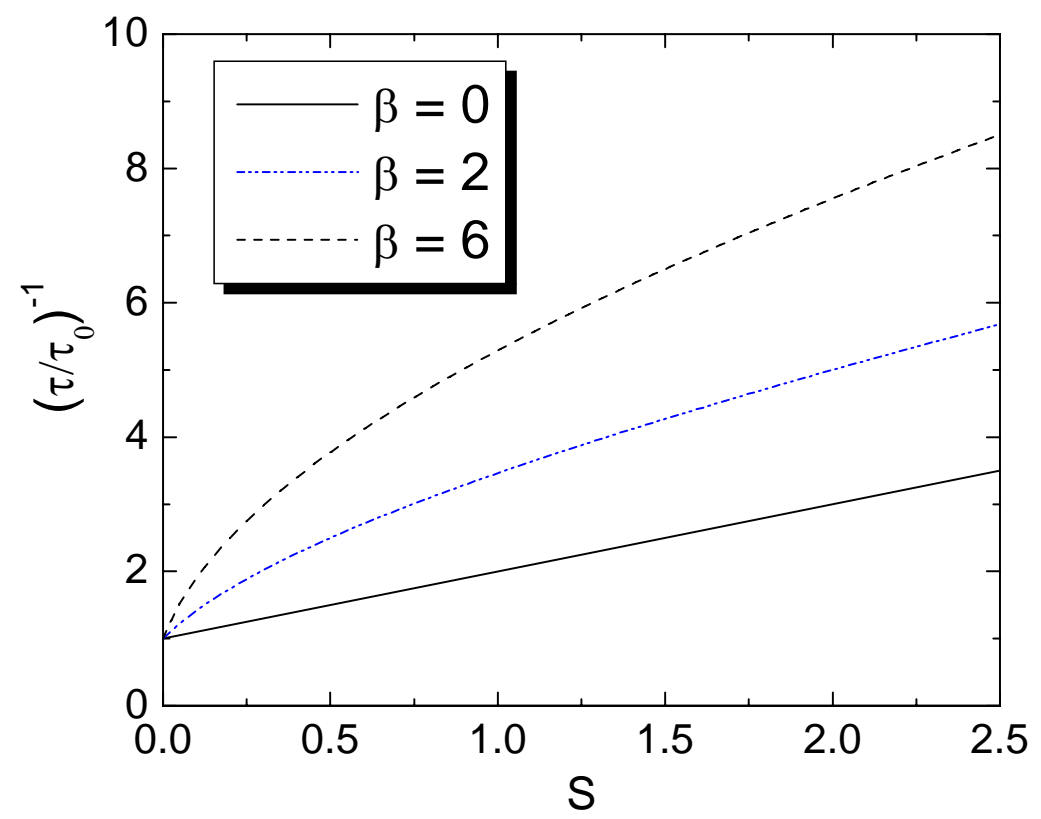

Figura 5-18 - Comportamento do tempo característico da não linearidade como função do parâmetro de saturação, $S$, para diferentes valores de $\beta$.

Claramente nota-se que para $\beta \neq 0$ a curva deixa de ter um comportamento linear com a intensidade incidente. Assim, a expressão para $\beta=0$ deve ser usada apenas quando o upconversion Auger for desprezível sob o risco de um resultado errôneo para $I_{S}$. Na Figura 5-19 podemos ver o comportamento de $\tau^{-1} x S$ ajustado com a Equação (5-25 para a fenda aberta. Deste gráfico obtivemos $\beta=5,3 \pm 1$ e $\tau_{0}=(240 \pm 20) \mu$ s que também está em bom acordo com os resultados previamente obtidos por Andrade [21]. Na mesma figura vemos o comportamento do tempo característico para o caso $\beta=0$ para comparação. Esta é uma forma alternativa de se obter $\gamma$ do comportamento transiente durante o regime de excitação $(S \neq 0)$, em contraposição à forma usualmente empregada realizada durante o regime de decaimento da população $(S=0)$. 


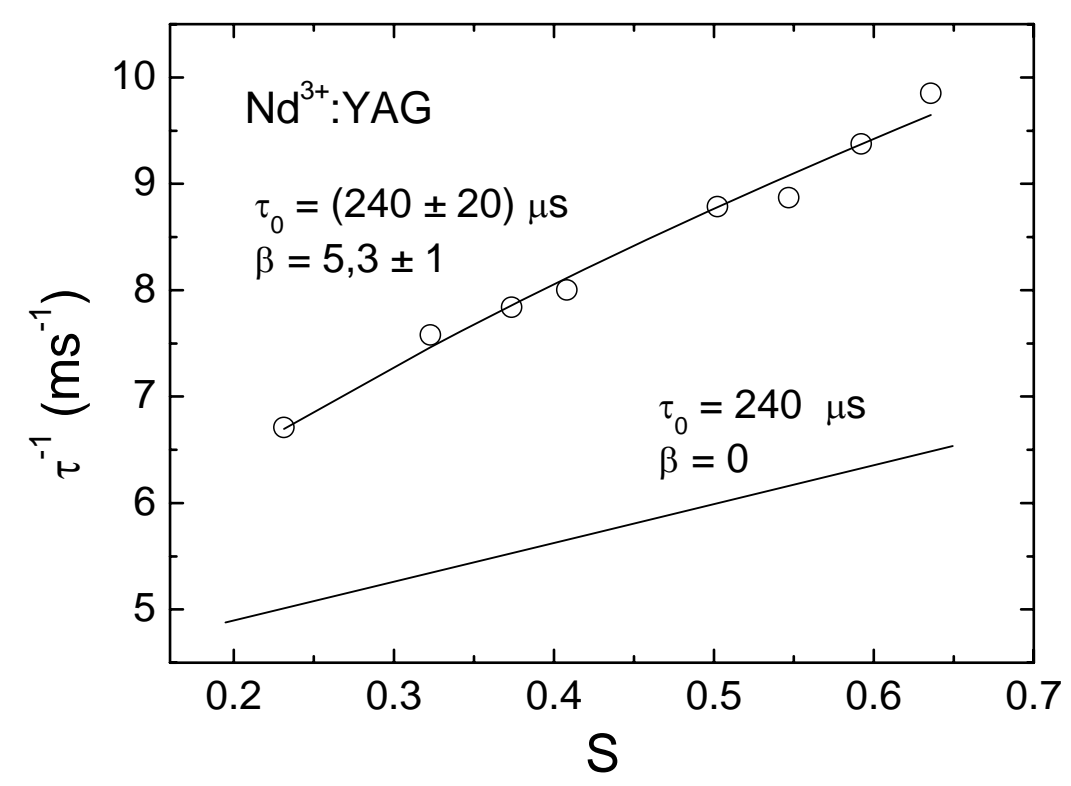

Figura 5-19 - Comportamento do tempo característico da não linearidade como função de $\mathrm{S}$ para a fenda aberta. No mesmo gráfico vemos o caso $\beta=0$.

\subsection{Conclusões do capítulo}

Neste capítulo foram apresentados resultados de medidas do parâmetro de upconversion Auger em materiais dopados com $\mathrm{Nd}^{3+}$ e $\mathrm{Cr}^{3+}$ usando a técnica de $z$-scan. Com isto aplicamos a técnica a novos materiais e expandindo sua aplicabilidade para o caso onde a excitação é feita em ressonância com o nível laser emissor. Esta técnica apresenta diversas vantagens quando comparada a outras encontradas na literatura. Além disso, obtivemos numericamente o comportamento da refração não linear como função do parâmetro de upconversion. Finalmente, derivamos uma expressão para o comportamento transiente da população do nível laser emissor, e do tempo de vida deste nível como função do parâmetro de upconversion. Estas expressões permitiram uma forma alternativa de calcular $\gamma$. 


\subsection{Referências}

[1] D. Jaque, O. Enguita, U. Caldino, M. O. Ramirez, J. Garcia Sole, C. Zaldo, J. E. MunozSantiuste, A. D. Jiang, and Z. D. Luo, Optical characterization and laser gain modeling of a NdAl3(BO3)(4) (NAB) microchip laser crystal, J. App. Phys. 90 (2001) 561-569.

[2] Z. D. Luo, Y. D. Huang, M. Montes, and D. Jaque, Improving the performance of a neodymium aluminium borate microchip laser crystal by resonant pumping, Appl. Phys. Lett. 85 (2004) $715-717$.

[3] C. Jacinto, T. Catunda, D. Jaque, and J. G. Sole, Fluorescence quantum efficiency and Auger upconversion losses of the stoichiometric laser crystal NdAl[sub 3](BO[sub 3])[sub 4], Phys. Rev. B 72 (2005) 235111.

[4] T. Y. Fan and R. L. Byer, Continuous-Wave Operation of a Room-Temperature, Diode-LaserPumped, 946-Nm Nd-Yag Laser, Opt. Lett. 12 (1987) 809-811.

[5] W. P. Risk and W. Lenth, Room-Temperature, Continuous-Wave, 946-Nm Nd-Yag Laser Pumped by Laser-Diode Arrays and Intracavity Frequency Doubling to 473-Nm, Opt. Lett. 12 (1987) 993-995.

[6] P. Zeller and P. Peuser, Efficient, multiwatt, continuous-wave laser operation on the F-4(3/2)-I4(9/2) transitions of Nd : YVO4 and Nd : YAG, Opt. Lett. 25 (2000) 34-36.

[7] C. Czeranowsky, E. Heumann, and G. Huber, All-solid-state continuous-wave frequencydoubled Nd : YAG-BiBO laser with 2.8-W output power at 473 nm, Opt. Lett. 28 (2003) 432434.

[8] D. Jaque, O. Enguita, J. G. Sole, A. D. Jiang, and Z. D. Luo, Infrared continuous-wave laser gain in neodymium aluminum borate: A promising candidate for microchip diode-pumped solid state lasers, Appl. Phys. Lett. 76 (2000) 2176-2178. 
[9] M. Montes, C. de las Heras, and D. Jaque, Passive Q-switching of a diode pumped Nd3+: CGGG crystal: Benefits of inhomogeneous line broadening and short pulse generation, Opt. Mat. 28 (2006) 408-414.

[10] Y. Guyot, H. Manaa, J. Y. Rivoire, R. Moncorge, N. Garnier, E. Descroix, M. Bon, and P. Laporte, Excited-State-Absorption and up-Conversion Studies of Nd3+-Doped-Single Crystals Y3al5o12, Ylif4, and Lamgal11o19, Phys. Rev. B 51 (1995) 784-799.

[11] S. Guy, C. L. Bonner, D. P. Shepherd, D. C. Hanna, and A. C. Tropper, High-inversion densities in Nd: YAG: Upconversion and bleaching, IEEE J. Quant. Elect. 34 (1998) 900-909.

[12] R. C. Powell, Physics of Solid-State Lasers Materials, Springer-Verlag, New York, 1998.

[13] S. A. Payne, G. D. Wilke, L. K. Smith, and W. F. Krupke, Auger up-Conversion Losses in NdDoped Laser Glasses, Opt. Comm. 111 (1994) 263-268.

[14] L. Meilhac, G. Pauliat, and G. Roosen, Determination of the energy diffusion and of the Auger upconversion constants in a Nd : YVO4 standing-wave laser, Opt. Comm. 203 (2002) 341-347.

[15] V. Pilla, T. Catunda, H. P. Jenssen, and A. Cassanho, Fluorescence quantum efficiency measurements in the presence of Auger upconversion by the thermal lens method, Opt. Lett. 28 (2003) 239-241.

[16] C. Jacinto, S. L. Oliveira, T. Catunda, A. A. Andrade, J. D. Myers, and M. J. Myers, Upconversion effect on fluorescence quantum efficiency and heat generation in Nd3+-doped materials, Opt. Exp. 13 (2005) 2040-2046.

[17] V. Lupei, A. Lupei, N. Pavel, T. Taira, I. Shoji, and A. Ikesue, Laser emission under resonant pump in the emitting level of concentrated Nd: YAG ceramics, Appl. Phys. Lett. 79 (2001) 590592. 
[18] V. Lupei, G. Aka, and D. Vivien, Enhanced fundamental and self-frequency-doubling laser emission efficiency in F-4(3/2) directly pumped Nd-activated nonlinear crystals: The case of GdCa4O(BO3)(3), Appl. Phys. Lett. 81 (2002) 811-813.

[19] V. Lupei, N. Pavel, and T. Taira, Highly efficient continuous-wave 946-nm Nd: YAG laser emission under direct 885-nm pumping, Appl. Phys. Lett. 81 (2002) 2677-2679.

[20] Y. Sato, T. Taira, N. Pavel, and V. Lupei, Laser operation with near quantum-defect slope efficiency in Nd : YVO4 under direct pumping into the emitting level, Appl. Phys. Lett. 82 (2003) 844-846.

[21] A. A. C. Andrade, Aplicações das técnicas de Lente Térmica e Z-scan ao estudo de sólidos dopados, Tese de Doutorado, Instituto de Física de São Carlos -USP, São Carlos, 2002.

[22] Y. F. Chen, C. C. Liao, Y. P. Lan, and S. C. Wang, Determination of the Auger upconversion rate in fiber-coupled diode end-pumped $N d: Y A G$ and $N d: Y V O 4$ crystals, App. Phys. B 70 (2000) 487-490.

[23] Y. F. Chen, L. J. Lee, T. M. Huang, and C. L. Wan, Study of high-power diode-end-pumped Nd : YVO4 laser at 1.34 mu m: influence of Auger upconversion, Opt. Comm. 163 (1999) 198-202.

[24] A. A. Kaminskii, Laser Crystals - Their Physics and Properties, Springer Verlag, New York, 1989.

[25] J. D. Zuegel and W. Seka, Upconversion and reduced F-4(3/2) upper-state lifetime in intensely pumped Nd : YLF, App. Opt. 38 (1999) 2714-2723. 


\section{Capítulo 6}

\section{Espectroscopia eletrônica em amostra vítrea dopada com $\mathrm{Yb}^{3+}$}

Com o advento dos lasers de alta potência de InGaAs emitindo em torno de 975 $\mathrm{nm}$ houve um crescente interesse em sistemas lasers dopados com $\mathrm{Yb}^{3+}$. Um bom exemplo deste interesse é a evolução das potências alcançadas em lasers de fibra dopadas com $\mathrm{Yb}^{3+}$ que desde meados de 1990 vem progredindo rapidamente de $2 \mathrm{~W}$ em 1995 [1], a $110 \mathrm{~W}$ em 1999 [2], chegando a $610 \mathrm{~W}$ em 2004 [3] e recentemente passando o limite de $1 \mathrm{KW}$ alcançando a marca de $1,36 \mathrm{KW}[4,5]$. Devido às altas potências alcançadas existe uma grande preocupação em se diminuir os efeitos térmicos neste meio [6,7]. Por outro lado, os efeitos da variação de fase induzidos devido à redistribuição de população também já foram pesquisados em fibra, onde se espera usar este efeito para chaveamento ótico [8]. Além de fibras, também as amostras bulk foram amplamente pesquisadas, principalmente cristais devido à alta condutividade térmica $[9$, 10]. No entanto, estes estudos focalizaram principalmente suas propriedades térmicas e seus efeitos. Nesta parte do nosso trabalho estaremos aplicando a técnica z-scan para estudar as propriedades eletrônicas de íons de $\mathrm{Yb}^{3+}$ em um vidro fosfato em função do comprimento de onda. Assim, a caracterização dos efeitos não lineares eletrônicos seria de grande relevância para este tipo de aplicação. Adicionalmente é feita uma comparação entre os efeitos térmico e eletrônico neste material para se determinar qual deles é o mais relevante. 


\subsection{Espectroscopia eletrônica no vidro $Q X / Y b$}

Como o efeito da carga térmica, $\phi$, é pequeno em materiais dopados com $\mathrm{Yb}^{3+}$, espera-se que a maior contribuição a $\Delta n$ seja proveniente do efeito de LP. Logo, determinar esta contribuição é de vital importância para o design de dispositivos óticos que utilizem este íon. Alguns trabalhos já foram realizados no sentido de se determinar a origem e a magnitude da não linearidade em materiais dopados com íons terra raras $[8$, 11-19]. No entanto, espectros não lineares foram obtidos apenas para os íons de $\mathrm{Er}^{3+}[8]$, $\mathrm{Nd}^{3+}[19] \mathrm{e} \mathrm{Cr}^{3+}$ [18]. De modo que, um espectro não linear do íon de $\mathrm{Yb}^{3+}$ viria a ajudar a compreender os mecanismos que dão origem ao índice de refração não linear neste íon. Além disto, este espectro permitiria calcular a variação do índice de refração como função do comprimento de onda, $\Delta n(\lambda)$ o que é importante para o design de lasers e sistemas de chaveamento ótico. Assim, o objetivo principal deste trabalho é determinar a forma de linha eletrônica de uma amostra dopada com $\mathrm{Yb}^{3+}$ usando a técnica de z-scan e, em conjunção com os dados da seção anterior, determinar a magnitude das contribuições devido a LT e a LP sobre a variação total de índice de refração.

Em sistemas intensamente bombeados, uma grande diminuição da população do estado fundamental pode ser alcançada. Como já dito anteriormente esta redistribuição de população muda a susceptibilidade ótica e consequentemente o índice de refração do meio. Em sólidos dopados com $\mathrm{Nd}^{3+}$ a não linearidade se origina principalmente de transições em altas energias, usualmente no ultravioleta, que se originam das camadas $4 f \rightarrow 5 d$. No caso de materiais dopados com $\mathrm{Cr}^{3+}$ o mecanismo da não linearidade é o mesmo, no entanto ela se origina da banda de transferência de carga ${ }^{1}$. Embora estejam

\footnotetext{
${ }^{1}$ São níveis no UV com alta força do oscilador (tipicamente $\sim 0,2$ ) correspondentes a processos onde o elétron é transferido de um ânion da rede para o íon de impureza.
} 
no ultravioleta, elas apresentam força do oscilador $\sim 10^{4}$ maior que as transições no visível. Assim, a susceptibilidade, e consequentemente o índice de refração não linear, tem sua maior contribuição destas transições no UV.

Para entender como estas transições afetam o índice de refração nos outros comprimentos de onda, devemos lembrar que em qualquer meio absortivo o índice de refração varia consideravelmente na vizinhança de uma absorção como função do comprimento de onda, como descrito através das relações de Kramers-Krönig. Consideremos, por simplicidade, a absorção com uma forma de linha Lorentziana (como é o caso para um sistema de dois níveis [20]), a refração terá uma forma de linha dispersiva, Figura 6-1.

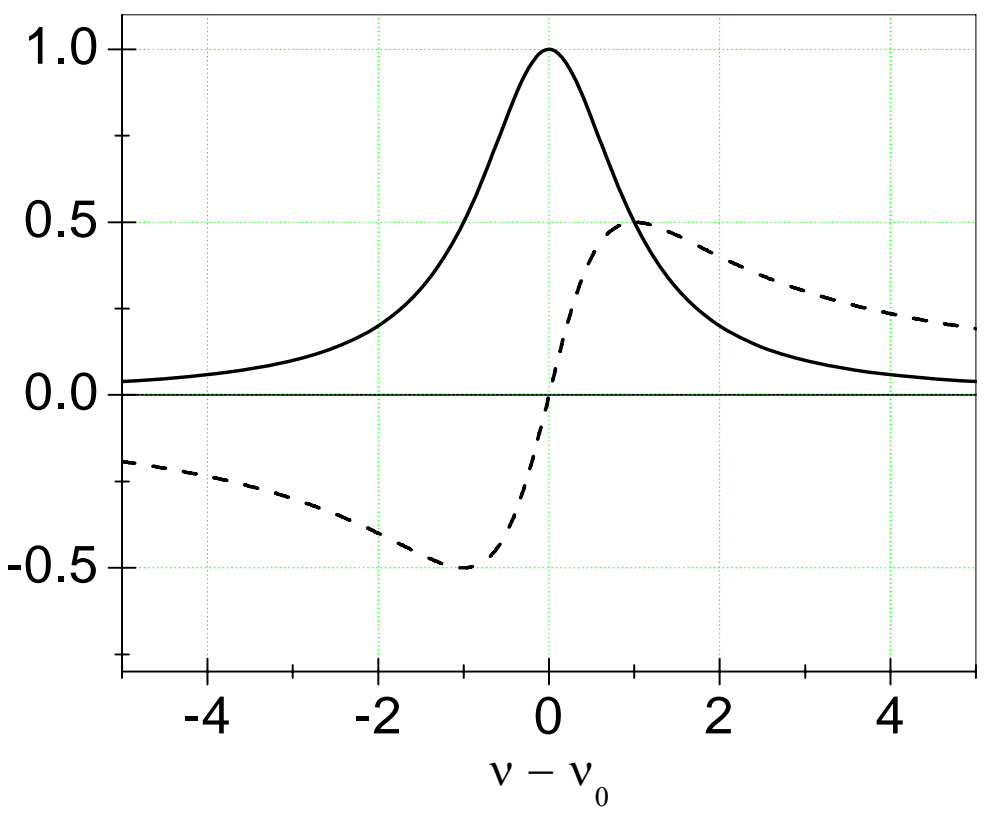

Figura 6-1 - Forma de linha absortiva (linha cheia) e dispersiva (tracejada) na vizinhança de uma absorção.

Como pode ser visto na figura acima, a absorção cai mais rapidamente que a refração quando a freqüência se afasta do centro da banda de absorção, $v_{0}$. Por isso, é possível que o índice de refração em um dado comprimento de onda seja afetado por diferentes transições, mesmo estando elas centradas em partes diferentes do espectro. Neste caso, o quanto cada uma destas transições irá afetar o índice de refração está 
relacionado com a força de cada transição (que é dada por sua força do oscilador $f$ ) e a sua desintonia em relação ao centro da transição estudada.

Alguns trabalhos já foram feitos para a determinação do efeito da não linearidade eletrônica em fibras dopadas com $\mathrm{Yb}^{3+}$ e outros íons terras-raras [11-14], usando a relação entre as transições do estado fundamental para os demais níveis e do estado excitado pelo feixe incidente para os níveis acima deste. $\mathrm{O}$ interesse neste caso era quantificar diretamente a diferença de fase induzida no feixe devido à não linearidade do íon, o que foi feito através de medidas interferométricas. Com isso, um razoável acordo foi encontrado entre os dados experimentais e o modelo teórico para o caso de excitação em 980 nm e prova em diversos comprimentos de onda, Figura 6-2.

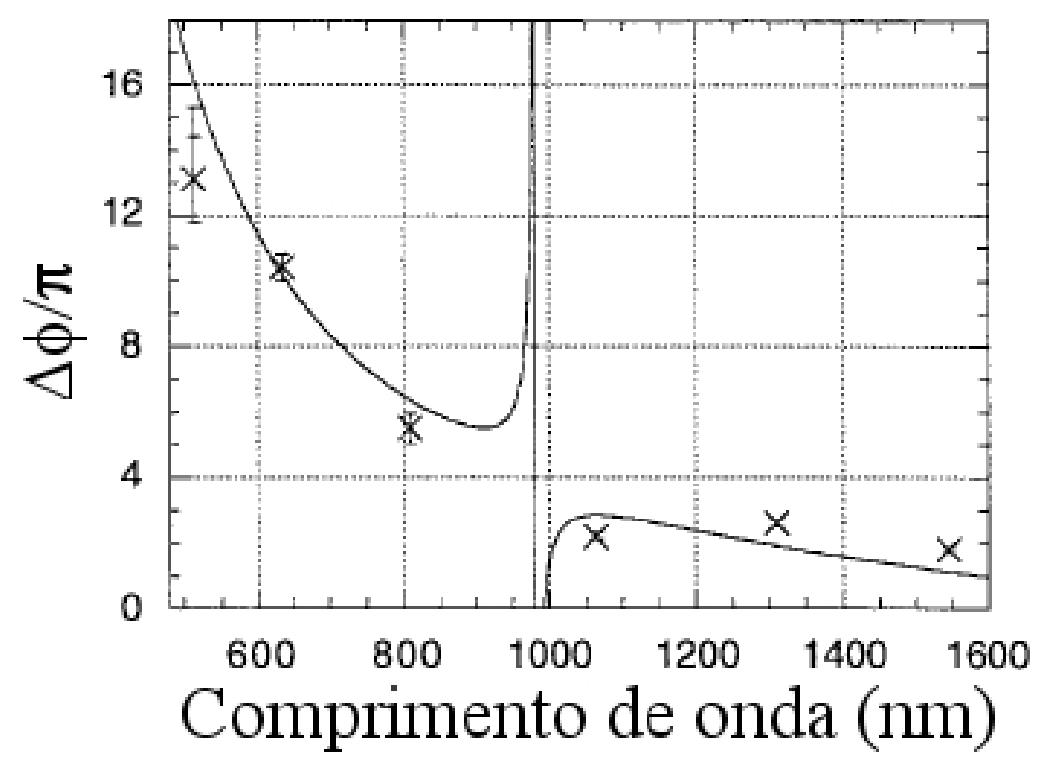

Figura 6-2 - Mudança de fase numa fibra de sílica dopada com $\mathrm{Yb}^{3+}$, em unidades de $\pi$. A fibra foi excitada em $980 \mathrm{~nm}$ e provada em diversos comprimentos de onda [13].

Entretanto, a força do oscilador de cada transição não é uma quantidade facilmente mensurável e muitas aproximações tiveram de ser feitas, como por exemplo considerar todas as transições no UV com o mesmo valor de $f$. Este valor foi então usado como um parâmetro ajustável. Além disso, as posições dos níveis no UV não são 
bem conhecidas para a sílica dopada com $\mathrm{Yb}^{3+}$, de forma que foram utilizados os valores do íon livre obtidos de um gás.

Devido a todas estas aproximações, usar este método para determinar o valor de $n_{2}$ (ou $\Delta \alpha$ ) pode levar a erros consideráveis, principalmente se as mesmas suposições forem feitas para diferentes matrizes. Assim, uma outra abordagem que não necessite destas aproximações seria mais precisa na determinação dos parâmetros não lineares. Uma alternativa é a utilização da técnica de z-scan que permite a determinação direta do valor de $n_{2}$, onde já estão embutidos todos os valores de força do oscilador e posições dos níveis no UV. Quando a energia de bombeamento estiver em ressonância com um nível no visível ou infravermelho (que é tipicamente o caso para a maioria das aplicações de terras raras), a contribuição a $n_{2}$ devido às transições no UV é conhecida como contribuição não ressonante. A contribuição ressonante deve-se ao próprio nível bombeado, e pode ser descrita usando a abordagem de um sistema de dois níveis. Logo, o índice de refração total deve ser descrito considerando estas duas contribuições.

Considerando inicialmente um sistema de dois níveis puros, onde $E_{e x}$ e $E_{g}$ são as energias para os níveis excitado e fundamental, respectivamente. Quando um feixe incide com freqüência $v=\left(E_{e x}-E_{g}\right) / h$ próxima à ressonância do sistema, faz com que a susceptibilidade comporte-se como [20]:

$$
\chi=-\frac{a_{o}}{2 \pi k} \frac{\delta-i}{\left(1+\delta^{2}+s\right)}
$$

onde $\delta=2 \pi\left(v-v_{o}\right) T_{2}$ o parâmetro de desintonia, com $T_{2}$ sendo à taxa de relaxação transversa, $v\left(v_{o}\right)$ a freqüência incidente (de ressonância), $a_{o}=\sigma_{g}\left(N_{e x}-N_{g}\right) n_{\delta} / 2 f_{L}^{2}$ é o coeficiente de atenuação do campo no centro da linha (definido como $\left.\alpha_{\mathrm{abs}}(\delta=0) / 2\right), n_{o}$ é o índice de refração, $S=I / I_{S}$ e $k=2 \pi\left(v_{o} / c\right)$ é o número de onda. Inicialmente a não linearidade em sólidos dopados foi tratada considerando apenas esta contribuição 
ressonante. Entretanto, como visto no Capítulo 3, devido a redistribuição de população tornou-se necessário adicionar à susceptibilidade o termo não ressonante, para se poder explicar os fenômenos observados. Desta forma a susceptibilidade total de uma amostra dopada com íons emissores excitada ressonantemente adquire a forma:

$$
\chi=\chi_{m}+\chi_{g}+\left(\chi_{e x}-\chi_{g}\right) \frac{N_{e x}}{N_{t}}-\frac{a_{o}}{2 \pi k} \frac{\delta-i}{\left(1+\delta^{2}+s\right)}
$$

com $\chi_{m}, \chi_{g}$ e $\chi_{e x}$ sendo as susceptibilidades da matriz, do estado fundamental e do estado excitado, respectivamente, e $N_{t}=N_{e x}+N_{g}$ a concentração total de íons na amostra. A partir de equações de taxa, ${ }^{2}$ pode-se calcular a população do estado excitado como:

$$
N_{e x}=N_{t} \frac{s}{2\left(1+\delta^{2}+s\right)}
$$

Substituindo a Equação (6-3 na (6-2 obtemos assim a susceptibilidade total do sistema:

$$
\chi=\chi_{m}+N_{t} \alpha_{g}+\frac{a_{o}}{2 \pi k}\left(\frac{A s-\delta+i}{1+\delta^{2}+s}\right)
$$

com

$$
A=\frac{4 \pi^{2}}{\lambda} \frac{f_{L}^{2}}{n_{o}} \frac{\Delta \alpha}{\sigma_{g}} .
$$

O fator $A$ introduz o efeito não ressonante da diferença de polarizabilidade, $\Delta \alpha$, na susceptibilidade, de modo que para $A=0$ a susceptibilidade volta a ser de um sistema

\footnotetext{
${ }^{2}$ Lembrando que para um sistema de dois níveis $\sigma(\delta)=\sigma_{0} /\left(1+\delta^{2}\right)$, com $\sigma_{0}$ a seção de choque de absorção no centro da linha.
} 
dois níveis. Usando $n_{2}=\frac{2 \pi f_{L}^{2}}{n_{o} I_{s}} \frac{\partial \chi}{\partial s}$, temos que o índice de refração não linear pode então ser calculado da seguinte forma:

$$
n_{2}=\frac{a_{o} f_{\mathrm{L}}^{2}}{n_{o} k \mathrm{I}_{\mathrm{s}}} \frac{A\left(1+\delta^{2}\right)+\delta-i}{\left(1+\delta^{2}+s\right)^{2}}
$$

Logo variando a freqüência incidente em torno da freqüência ressonante é possível se obter o espectro de $n_{2}$ em torno desta transição. Na verdade, o efeito de $\Delta \alpha$ sobre as interações não lineares ressonantes foram teoricamente consideradas por Butylkin et al [21], de modo que a Equação (6-2) pode ser obtida de uma equação mais geral, considerando que $\tau \gg T_{2}$. Em geral este limite é bem satisfeito sólidos dopados com íons emissores onde, à temperatura ambiente, $\tau \sim$ ms e $T_{2} \sim$ ps.

\subsubsection{Aparato experimental da espectroscopia não linear}

Para obter o espectro não linear de amostras dopadas com íons de $\mathrm{Cr}^{3+}$, Lima et al [18] utilizaram uma adaptação da técnica de z-scan resolvida no tempo. Este arranjo está descrito na Figura 6-3. Inicialmente é feita uma medida normal de z-scan para determinar as posições de pico e vale da curva da fenda fechada. Logo após a amostra é colocada na posição de máxima variação da transmitância (posição de pico ou vale), e a freqüência do laser de excitação é então continuamente sintonizada em torno da transição estudada. 


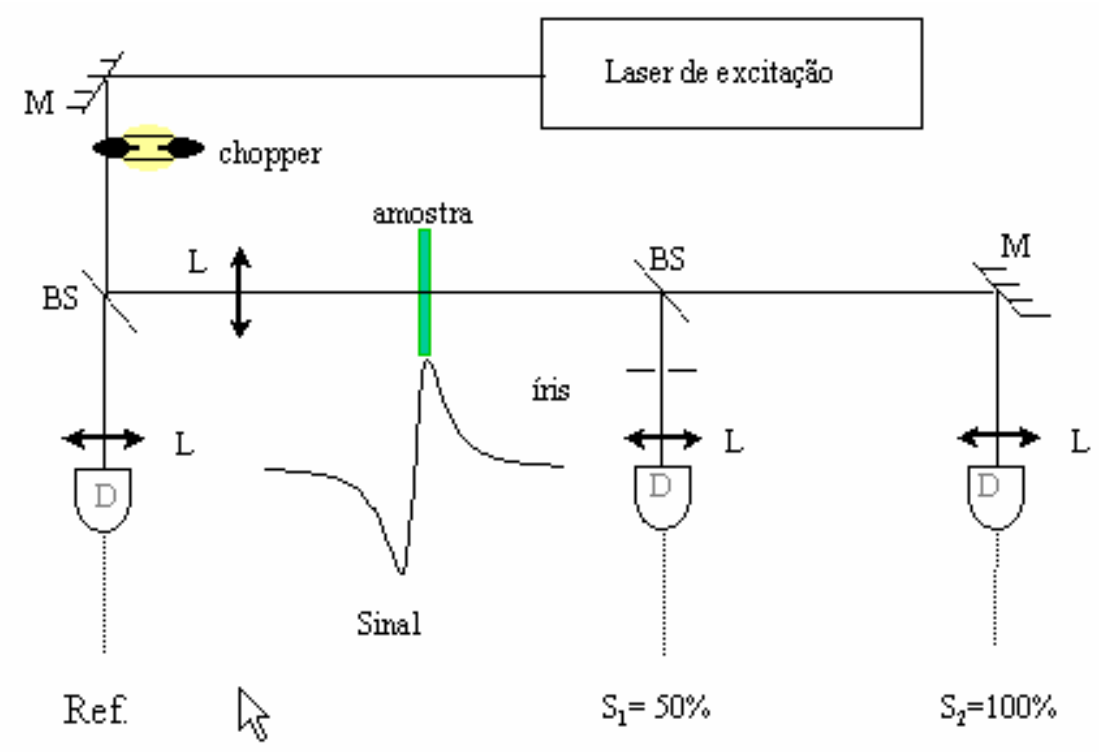

Figura 6-3 - Aparato experimental usado para medidas de Z-scan e espectroscopia. $\mathrm{M}=$ espelho, $\mathrm{L}=$ lente, $\mathrm{BS}=$ divisor de feixe, $\mathrm{D}=$ detector, $\mathrm{S}_{1}$ e $\mathrm{S}_{2}$ as transmitâncias da íris à frente dos detectores, Ref é o sinal de referência usado para obter a transmitância linear da amostra.

A seguir é obtido um espectro de absorção linear, $\alpha_{a b s}(\lambda)$, do material varrendo-se a freqüência do laser de excitação e fazendo a razão entre a potência da luz que é transmitida pela amostra e a que chega ao detector de referência. Com este espectro podemos calcular o comportamento de $L_{e f}(\lambda)$ como função de $\lambda$ (lembrando que $\left.L_{e f}=\left(1-\exp \left(-\alpha_{\mathrm{abs}}(\lambda) L\right)\right) / \alpha_{\mathrm{abs}}(\lambda)\right)$. Esta medida é importante pois as partes real e imaginária do índice de refração não linear são proporcionais a razão entre a variação da transmitância e o comprimento efetivo da amostra, tanto para a fenda aberta como para a fechada.

Como o $\mathrm{Yb}^{3+}$ também é um meio com não linearidade lenta $(\tau \sim \mathrm{ms})$, podemos utilizar a técnica de z-scan resolvido no tempo para estudar o comportamento da não linearidade com a freqüência, levando em conta que o sinal da fenda fechada (fator de abertura 50\%) é proporcional à parte dispersiva do índice de refração $\left(\propto n_{2}\right)$ enquanto que o da fenda aberta $(100 \%)$ é proporcional à parte absortiva $\left(\propto n_{2}\right.$ ”). Logo, para um tempo inicial $t_{1}<<\tau_{0}$ apenas efeitos lineares existem no sinal transiente da 
transmitância, ao passo que para um tempo $t_{2}>\tau_{0}$ tanto efeitos lineares como não lineares estão presentes no sinal transiente. Desta forma podemos eliminar os efeitos lineares fazendo a normalização $I(v, t) / I(v, 0)$, ficando a transmitância proporcional apenas aos efeitos não lineares. Experimentalmente, esta relação entre a transmitância e o índice de refração não linear é feita através da Equação 3-28 para a parte real e da Equação 3-30 para a parte imaginária, da seguinte maneira:

$$
n_{2}{ }^{\prime}=\frac{\lambda}{2 \pi I_{o} 0,406(1-S)^{0.25}} \frac{\Delta T_{p v}}{L_{e f}}
$$

e

$$
n_{2} "=\frac{\lambda}{2 \pi I_{o}} \frac{(T-1)}{L_{e f}}
$$

onde $T$ é a transmitância da fenda aberta na posição de máximo (mínimo) para a fenda fechada. É importante ressaltar que estas medidas são realizadas com baixa intensidade para evitar os efeitos de saturação descritos no Capítulo 4.

\subsubsection{Determinação de $I_{S}$ e $\tau_{0}$}

Inicialmente foram realizadas medidas de refração transiente, no pico da banda de absorção em $976 \mathrm{~nm}$ usando um laser de Ti:safira, para a determinação da intensidade de saturação, $I_{S}$, e do tempo de vida do nível, $\tau_{0}$. A Figura 6-4(a) exibe um transiente típico obtido, que foi ajustado por um decaimento exponencial. Já na Figura 6-4(b) está exibido o comportamento de $\tau^{-1} \times I$. Do ajuste dos dados experimentais foram obtidos $I_{S}=4,3 \mathrm{KW} / \mathrm{cm}^{2}$ e $\tau_{0}=2,4 \mathrm{~ms}$. Este valor de $\tau_{0}$ está próximo ao valor obtido de medidas diretas de decaimento da fluorescência, $\tau_{\text {exp }} \sim 2,3 \mathrm{~ms}$. Além disso, usando a relação $I_{S}=h v_{d} \sigma_{g} \tau_{o}$ e $\sigma_{g}=1.9 \times 10^{-20} \mathrm{~cm}^{2}$ (calculado de $\sigma_{g}=\alpha_{\mathrm{abs}} / \mathrm{N}_{\mathrm{t}}$ ), nós calculamos 
$I_{S}=4.6 \mathrm{KW} / \mathrm{cm}^{2}$, que está em excelente acordo com o valor experimental. A boa concordância entre estes resultados é uma indicação de que a concentração nominal de $\mathrm{Yb}^{3+}$ está próxima do seu valor real.

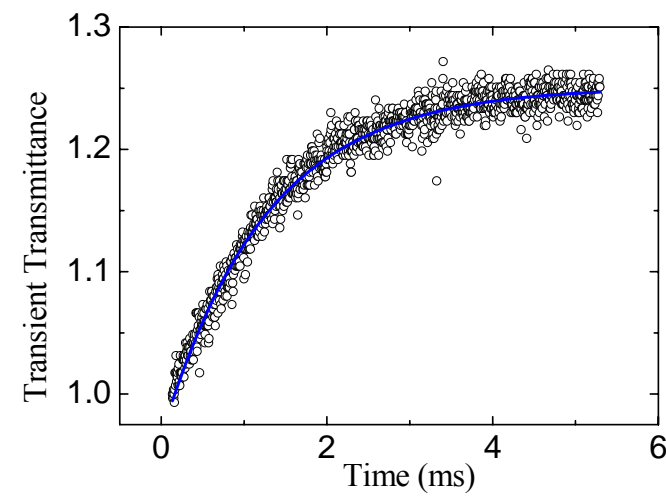

(a)

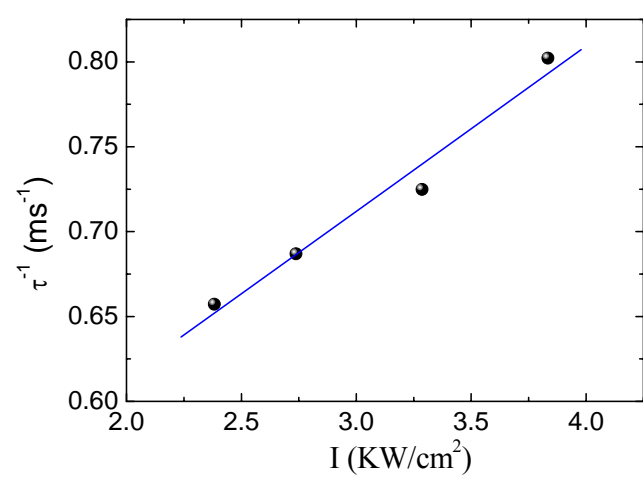

(b)

Figura 6-4 - Determinação dos parâmetros $I_{S}$ e $\tau_{0}$ através da técnica de refração transiente. (a) Sinal transiente transmitido pela amostra. (b) Inverso do tempo de resposta da não linearidade como função da intensidade incidente. Do ajuste foram obtidos: $I_{S}=4,3 \mathrm{KW} / \mathrm{cm}^{2}$ e $\tau_{0}=2,4 \mathrm{~ms}$.

\subsubsection{Determinação do espectro de $n_{2}$}

A seguir foi realizada uma medida convencional de z-scan, em $976 \mathrm{~nm}$, para se determinar o valor do parâmetro $A$. Desta medida o índice de refração não linear, $n_{2}$, foi obtido como $n_{2}=(2,5+\mathrm{i} 2,3) \times 10^{-8} \mathrm{~cm}^{2} / \mathrm{W}$ (lembrando que por definição $n_{2}=n_{2}$ '- in $\left.{ }_{2}{ }^{\prime}\right)$.

Assim, como $A=\frac{n_{2}{ }^{\prime}(\delta=0)}{n_{2}{ }^{\prime}(\delta=0)}$ (vide Equação (6-6) calculamos $A=1,06$. Usando agora a Equação 3-11 a diferença de polarizabilidade foi calculada como $\Delta \alpha=2,3 \times 10^{-26} \mathrm{~cm}^{3}$. Dos dados de Arkwright et al[13], para fibra de sílica dopada com $\mathrm{Yb}^{3+}$, nós calculamos $\Delta \alpha \sim 2,8 \times 10^{-26} \mathrm{~cm}^{3}$, o que está próximo ao nosso valor encontrado experimentalmente. 


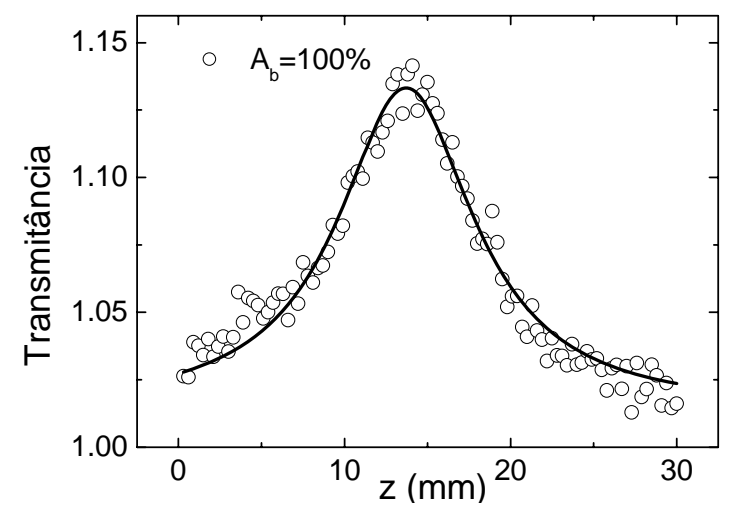

(a)

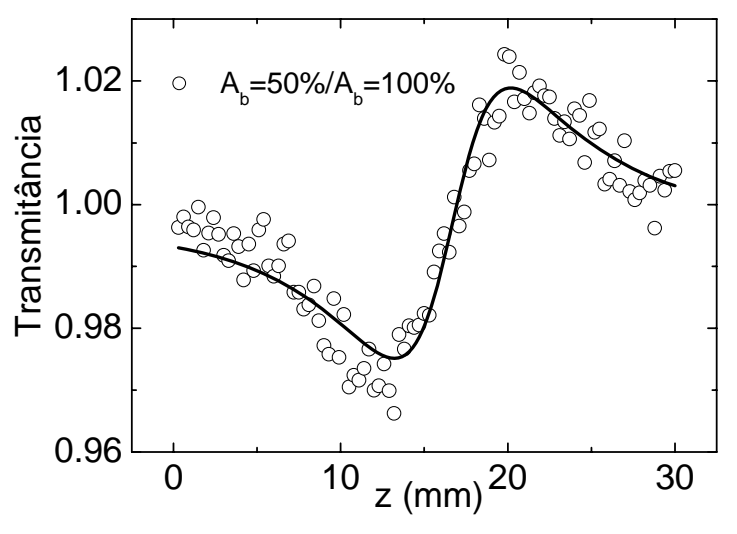

(b)

Figura 6-5 - Medidas de z-scan no vidro $\mathrm{QX} / \mathrm{Yb}$ para $\lambda$ no pico da linha de absorção. (a) Curva de saturação, $A_{b}=100 \%$ e (b) curva de refração $A_{b}=50 \% / A_{b}=100 \%$.

A seção de choque de absorção encontrada, através da Equação 3-12, foi $\Delta \sigma=$ $2.2 \times 10^{-20} \mathrm{~cm}^{2}$. Este valor está em excelente concordância (em módulo) com a seção de choque de absorção do estado fundamental, $\sigma_{g}=1.92 \times 10^{-20} \mathrm{~cm}^{2}$. Esta concordância já era esperada pois não há absorção de estado excitado para este comprimento de onda, de modo que $\Delta \sigma=-\sigma_{g}$.

Para confirmar este último resultado, nós calculamos a taxa de variação do coeficiente de absorção, no centro da linha, de duas maneiras independentes: Primeiro, como o único nível no intervalo visível-infravermelho é o ${ }^{2} \mathrm{~F}_{5 / 2}$, é esperado que o coeficiente de absorção sature como:

$$
\alpha_{a b s}=\frac{\alpha_{a b s}(\delta=0)}{1+I / I_{S}}
$$

Por outro lado, como a intensidade incidente na amostra decai com $\exp \left(-\alpha_{a b s} z\right)$, onde $\alpha_{\mathrm{abs}}=2 k\left(n_{0}{ }^{\prime}+n_{2}{ }^{\prime} I\right)$. Logo, é possível demonstrar que:

$$
\left.\frac{d \alpha_{a b s}}{d I}\right|_{I=0}=-\frac{\alpha_{a b s}(0)}{I_{S}}=2 k n_{2}^{\prime \prime}
$$

Onde $\alpha_{a b s}(0)=11,2 \mathrm{~cm}^{-1}$ é o coeficiente de absorção no centro da linha. Usando então o valor de $n_{2}$ ” obtido anteriormente, chegamos a $I_{S}=3,8 \mathrm{KW} / \mathrm{cm}^{2}$. Este resultado está em 
bom acordo com aquele encontrado nas medidas transientes, $I_{S}=4,3 \mathrm{KW} / \mathrm{cm}^{2}$. estes valores de $\mathrm{I}_{\mathrm{S}}$ são aproximadamente o dobro do valor teórico para um sistema de dois níveis, $I_{S}=h v / 2 \sigma \tau=2,3 \mathrm{KW} / \mathrm{cm}^{2}$. Esta discrepância indica que o sistema é melhor modelado por um sistema de quatro níveis, onde a absorção não está em ressonância com a emissão e $I_{S}=h v / \sigma \tau$.

Após estas medidas preliminares, foi então determinado o espectro de $n_{2}$ do vidro QX/Yb. Como a banda de absorção se estende por mais de $100 \mathrm{~nm}$, resolvemos fazer um espectro ponto-a-ponto, iniciando em $900 \mathrm{~nm}$ e incrementando o comprimento de onda em aproximadamente $10 \mathrm{~nm}$ a cada ponto. Cada um destes pontos é uma medida de z-scan convencional. O espectro obtido está exibido na Figura 6-6. Podemos notar desta figura que, como esperado, $n_{2}{ }^{\prime \prime}(\lambda)$ vai a zero mais rápido que $n_{2}{ }^{\prime}(\lambda)$.

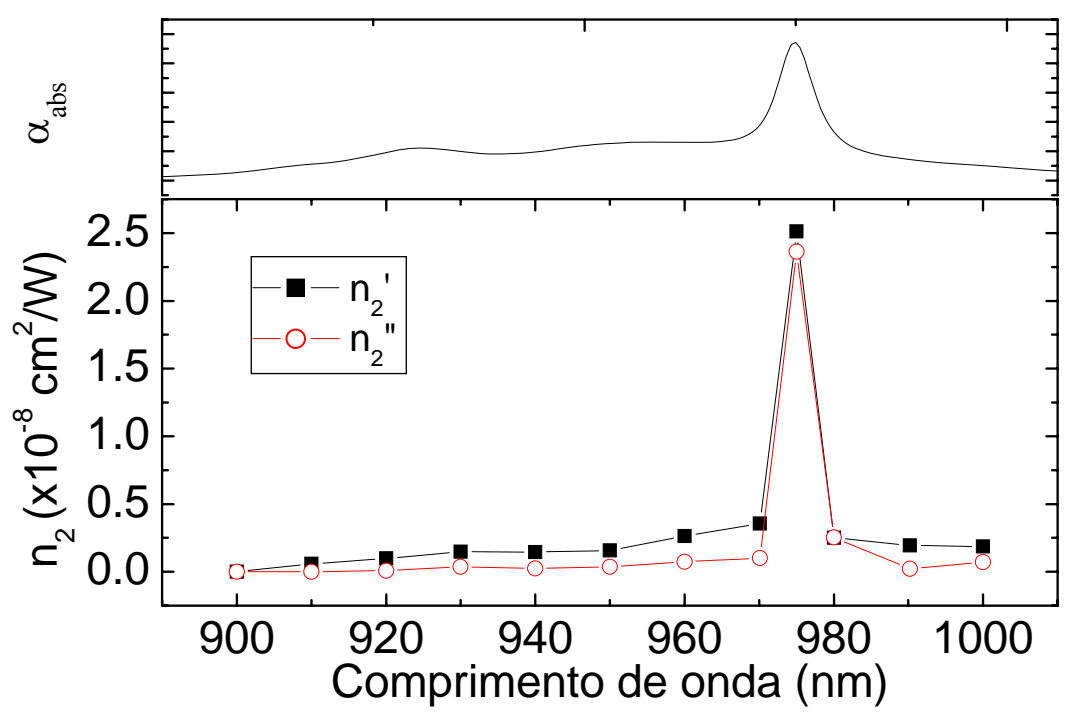

Figura 6-6 - Espectro não linear do $\mathrm{Yb}^{3+}$ no vidro QX obtido com medidas convencionais de z-scan. $\mathrm{Na}$ parte superior pode-se ver o espectro de absorção linear, para comparação.

Outra característica importante nesta figura é a forma Lorentziana da parte real de $n_{2}$. Ela indica que a contribuição não ressonante à não linearidade é mais importante que a ressonante, ou seja, a não linearidade do $\mathrm{Yb}^{3+}$ é governada pelas transições no ultravioleta. Isto se deve ao fato da contribuição não ressonante ser proporcional a 
$N_{e x}(\delta) \Delta \alpha$, onde $N_{e x}(\delta)$ tem uma forma de linha Lorentziana, Equação (6-3. Isto é particularmente interessante para o $\mathrm{Yb}^{3+}$ pois sua estrutura de níveis é basicamente a de um sistema de dois níveis, que apresenta um caráter dispersivo.

A Figura 6-7 mostra o comportamento de $\Delta \alpha$ como função do comprimento de onda incidente. Os valores encontrados são comparáveis aos resultados obtidos na literatura para vidros fosfatos dopados com $\mathrm{Nd}^{3+}[22](2-3) \times 10^{-26} \mathrm{~cm}^{3}$ e também a $1,9 \times 10^{-26} \mathrm{~cm}^{3}$ recentemente obtido para o cristal de $\mathrm{Yb}^{3+}:$ YAG [23]. Como pode ser visto na figura abaixo, $\Delta \alpha(\lambda)$ é aproximadamente constante no intervalo de freqüências estudado. Isto ajuda a corroborar o caráter não ressonante de $\Delta \alpha$, como esperado no nosso modelo.

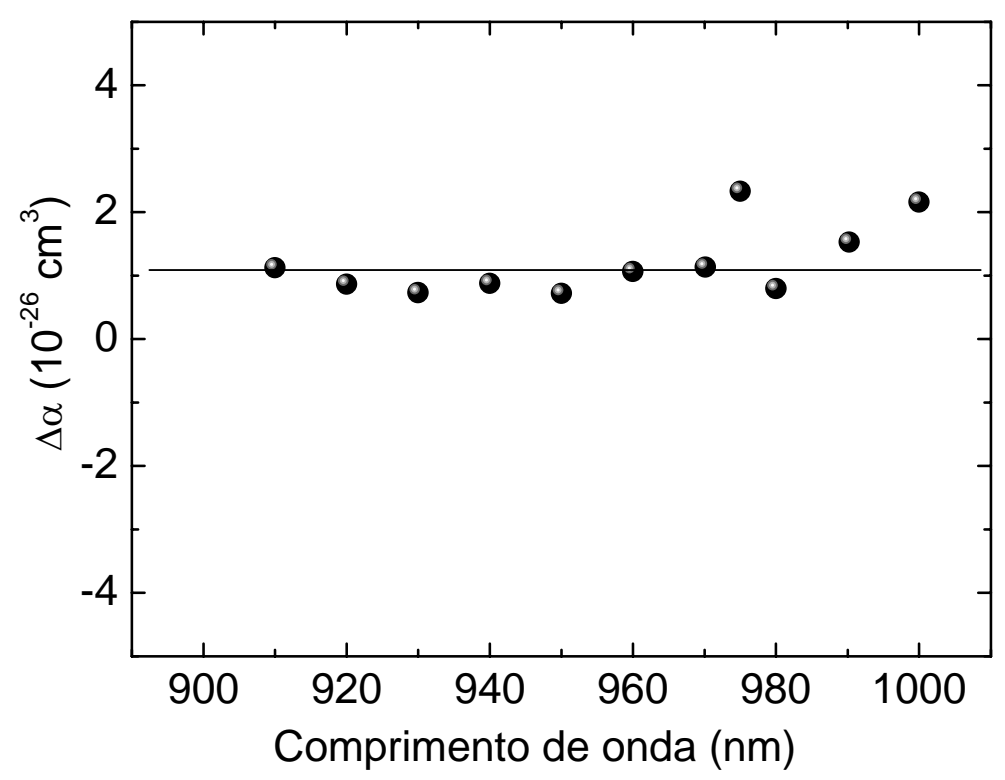

Figura 6-7 - Diferença de polarizabilidade como função do comprimento de onda incidente.

Finalmente, com a amostra fixa na posição de vale, o comprimento de onda do laser de Ti:safira foi variado em passos de $0,04 \mathrm{~nm}$ em torno do pico de absorção. A Figura 6-8 mostra o espectro de índice de refração não linear obtido. Além do caráter Lorentziano apresentado na Figura 6-8 para $n_{2}$, pode-se perceber que as posições de 
pico para $n_{2}$ ' e $n_{2}$ " estão em diferentes comprimentos de onda. Para compreender este comportamento é interessante analisar a Equação (6-6 para diferentes valores do parâmetro $A .^{3}$

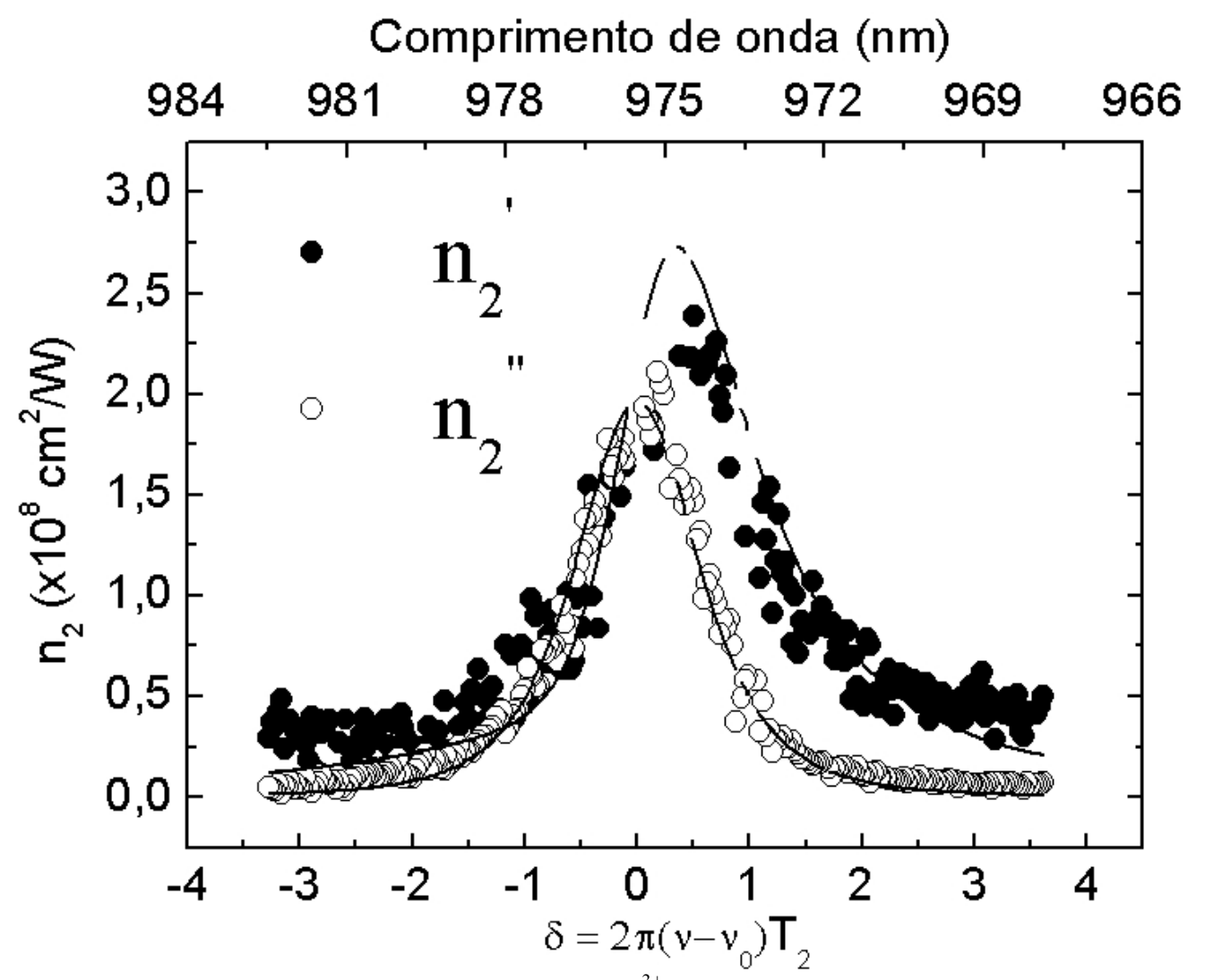

Figura 6-8 - Espectro não linear do $\mathrm{Yb}^{3+} \mathrm{em}$ torno do pico de absorção.

Nesta equação vemos que $n_{2}$ ” $(\lambda)$ é insensível à interferência entre as contribuições ressonante e não ressonante de $n_{2}$. Mas $n_{2}{ }^{\prime}(\lambda)$ sente este efeito através do parâmetro $A$. Assim, supondo $s=0$ na Equação (6-6 e variando $\delta$ para alguns valores de $A$ pode-se construir a Figura 6-9. Por esta figura nota-se que quando $A=0, n_{2}$ ' apresenta um caráter dispersivo enquanto que para maiores valores de $A$, como $A=3$, o comportamento passa a ser similar ao Lorentziano. Como dito antes, isto se deve ao fato da contribuição não-ressonante ser proporcional a $N_{e x} \Delta \alpha$. Para valores intermediários de A vemos um deslocamento da posição de pico de $n_{2}$ ', em direção ao centro da banda.

\footnotetext{
${ }^{3}$ Vale salientar que esta análise foi inicialmente feita para explicar o comportamento da não linearidade de materiais dopados com $\mathrm{Cr}^{3+}[18]$.
} 
Este comportamento é especialmente claro para $A=1$, que é o caso do íon de $\mathrm{Yb}^{3+}$ estudado. Portanto, o deslocamento visto entre as posições de pico de $n_{2}$ 'e $n_{2}$ ” pode ser atribuído a um efeito de interferência entre as duas contribuições a $n_{2}$. $\mathrm{O}$ valor do parâmetro $A$ pode também ser encontrado através do ajuste dos dados experimentais com a Equação (6-6, Figura 6-9. Neste caso, inicialmente ajustamos a parte imaginária de $n_{2}$ de onde obtivemos o valor do comprimento de onda no centro da linha, $\lambda_{0}=$ $975,13 \mathrm{~nm}$, e da largura da linha, $\Delta \lambda=2,22 \mathrm{~nm}$. Estes resultados foram então utilizados como parâmetros fixos para o ajuste da parte real de $n_{2}$. Deste ajuste foi então obtido o valor de $A \sim 0,8$, que está em razoável com o valor encontrado no centro da linha.

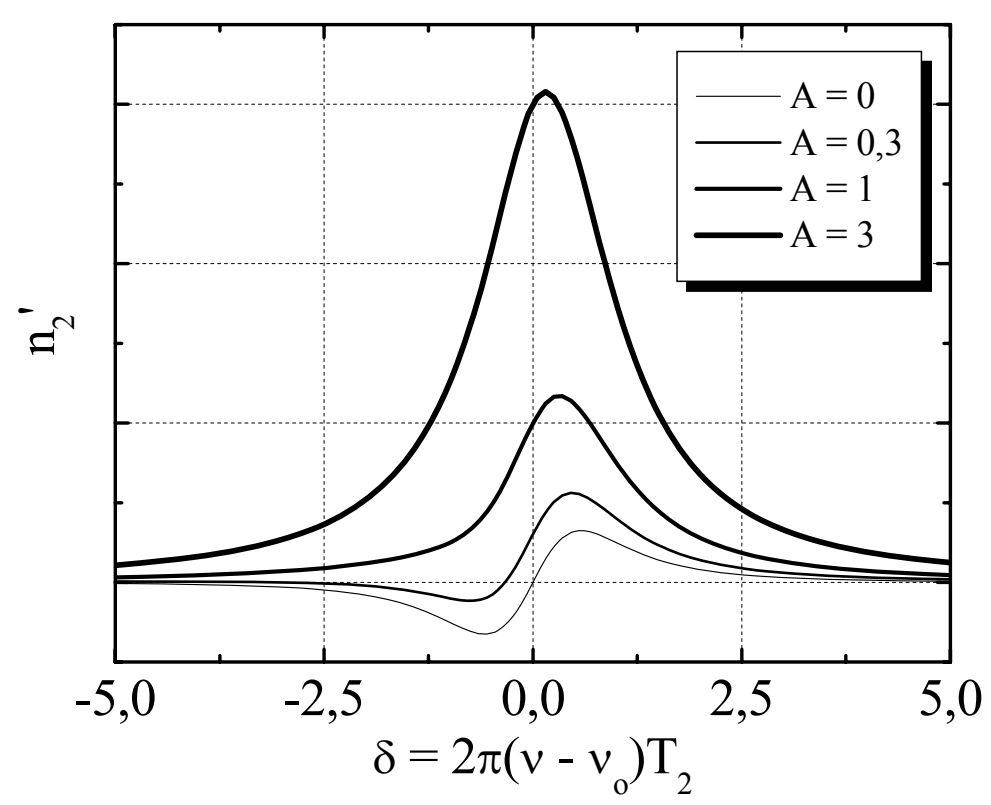

Figura 6-9 - Simulação da dependência da parte real do índice de refração não-linear, $n_{2}$ ', em função de $\delta$ para diferentes valores de $A$ [18].

Entretanto, se usarmos o valor $A=1,06$ para calcular o índice de refração não linear na Equação (6-6 obteremos um valor de $n_{2}=(1.3+1.2 \mathrm{i}) \times 10^{-8} \mathrm{~cm}^{2} / \mathrm{W}$. Este valor é bem diferente daquele obtido por uma medida de z-scan. Uma causa provável desta diferença pode ser relativa à Equação (6-6, que foi obtida para um sistema de dois níveis puro (embora seu comportamento seja mais próximo de um sistema de quatro 
níveis) e não leva em consideração o acoplamento térmico entre os subníveis. $\mathrm{Na}$ verdade, o nível ${ }^{2} \mathrm{~F}_{5 / 2}$ é um estado tripleto cujos subníveis superiores estão a $475 \mathrm{~cm}^{-1} \mathrm{e}$ $192 \mathrm{~cm}^{-1}$ distante do subnível inferior [24]. Além disto, a energia de fônon do vidro QX/Yb é de aproximadamente $1100 \mathrm{~cm}^{-1}$ [24], de modo que estes subníveis estão fortemente acoplados termicamente. Assim, para descrever corretamente a não linearidade do $\mathrm{Yb}^{3+}$ a (6-6 têm de ser modificada para considerar este acoplamento térmico.

\subsection{Determinação da magnitude das contribuições térmica e eletrônica}

Embora as contribuições térmicas e eletrônicas coexistam ao mesmo tempo, elas possuem magnitudes diferentes. Para estimar a magnitude relativa entre elas podemos tomar a razão entre as variações de fase induzidas pelo bombeamento, $\left|\Delta \phi_{\mathrm{LP}} / \Delta \phi_{\mathrm{LT}}\right|$.

Para o caso pulsado, podemos desconsiderar o efeito de difusão de calor. Seguindo o procedimento adotado por Powell et al [25] podemos escrever a variação de fase devido a lente de população como $\Delta \phi_{L P}=k L n_{2}$ 'I, com $n_{2}$ ' dado pela Equação 3-11. Mas para $I<<I_{S} N_{e x} \sim N_{t} I / I_{S}$. De modo que:

$$
\Delta \phi_{L P}=2 \pi f_{L}^{2} N_{e x} k L \Delta \alpha / n_{0}
$$

onde $f_{\mathrm{L}}$ é o fator Lorentz de correção do campo local e $k=2 \pi / \lambda$.

Enquanto que para a lente térmica teremos $\Delta n_{L T}=(d n / d T) \Delta T$, pois desconsiderando a difusão de calor temos $d n / d T \sim d s / d T$. Por outro lado, a variação de temperatura é dada por $\Delta T=N_{e x} \phi h v_{e x} / c_{p} \rho$ de forma que:

$$
\Delta \phi_{L T}=\left(N_{e x} h v_{e x} \phi k L / c_{p} \rho\right) d n / d T
$$


onde $\phi$ é a carga térmica do material, $c_{p}$ o calor específico, $\rho$ a densidade do material e $v_{\mathrm{ex}}$ a freqüência de excitação. Logo para obter a magnitude relativa entre as variações de fase induzidas pelos efeitos eletrônico e térmico no regime pulsado devemos fazer:

$$
\left|\frac{\Delta \phi_{L P}}{\Delta \phi_{L T}}\right|=\frac{2 \pi f_{L}^{2}}{n_{0}} \frac{c_{p} \rho}{h v_{e x} d n / d T} \frac{\Delta \alpha}{\phi}
$$

Substituindo os valores dos parâmetros fototérmicos e não lineares na Equação (6-13 obtivemos $\left|\Delta \phi_{\mathrm{LP}} / \Delta \phi_{\mathrm{LT}}\right| \sim 20$, o que implica dizer que o efeito térmico é aproximadamente vinte vezes menor que o eletrônico. Este valor está muito acima daquele encontrado por Powell et al para amplificadores óticos bombeados com lâmpada flash dopados com $\mathrm{Nd}^{3+}[25],\left|\Delta \phi_{\mathrm{LP}} / \Delta \phi_{\mathrm{LT}}\right| \sim 0,5$. A diferença está no defeito quântico dos íons de $\mathrm{Yb}^{3+}(\sim 3 \%)$ que é bem menor que o dos íons de $\mathrm{Nd}^{3+}(\sim 25 \%)$, e por isso geram bem menos calor. Portanto, a principal contribuição para a variação do índice de refração no vidro $\mathrm{QX} / \mathrm{Yb}$ é de origem eletrônica.

No regime contínuo devemos re-escrever a variação de fase devido a LT, levando agora em conta a difusão de calor. Lembrando que $\theta \cong \phi(r=\sqrt{2} w)-\phi(r=0) \propto \Delta T_{\theta}$ e que $\Delta \phi_{L T} \cong \phi(r=\infty)-\phi(r=0) \propto \Delta T_{T}$ no regime estacionário $(\mathrm{t} \rightarrow \infty) \theta$ podemos fazer uma estimativa da diferença de fase total como sendo $\Delta \phi_{L T} \cong \frac{\Delta T_{T}}{\Delta T_{\theta}} \theta$. Simulando numericamente o perfil radial de temperatura com os dados do $\mathrm{QX} / \mathrm{Yb}$ obtivemos $\Delta T_{T} / \Delta T_{\theta} \sim 3,86$, de modo que a razão entre as fases térmica e eletrônica será:

$$
\left|\frac{\Delta \phi_{L P}}{\Delta \phi_{L T}}\right| \cong \frac{4}{3,86 w_{0}^{2}} \frac{K}{\phi d s / d T} \frac{n_{2}^{\prime}}{\alpha_{a b s}}
$$


Desta forma temos que, para o vidro $\mathrm{QX} / \mathrm{Yb}$, no regime de excitação contínua $\left|\Delta \phi_{\mathrm{LP}} / \Delta \phi_{\mathrm{LT}}\right| \sim 8,45$. Este resultado evidencia a importância da difusão de calor no aumento dos efeitos térmicos.

\subsection{Conclusões do capítulo}

Neste Capítulo, nós apresentamos, pela primeira vez, medidas de forma de linha eletrônica em um material dopado com $\mathrm{Yb}^{3+}$ usando uma adaptação da técnica de $\mathrm{z}$ scan. Esta determinação da forma da linha permitiu discriminar qual a contribuição mais importante para a não linearidade dos íons de $\mathrm{Yb}^{3+}$. Foi visto que $n_{2}$ ' tem uma forma de linha Lorentziana, apesar do $\mathrm{Yb}^{3+}$ ter uma estrutura de níveis semelhante a um sistema de dois níveis. Devido à própria forma do espectro ficou evidente que a maior contribuição à não linearidade refrativa vem das transições no ultravioleta, tendo a contribuição ressonante um peso menor. Isto reforça a idéia de que o mecanismo que domina a não linearidade de materiais dopados com terras raras é basicamente o mesmo. Finalmente, foi possível estimar que a LP é sempre maior que a LT, seja no caso de bombeamento pulsado ou contínuo. 


\subsection{Referências}

[1] H. M. Pask, R. J. Carman, D. C. Hanna, A. C. Tropper, C. J. Mackechnie, P. R. Barber, and J. M. Dawes, Ytterbium-Doped Silica Fiber Lasers - Versatile Sources for the 1-1.2 Mu-M Region, IEEE J. Sel. Top. Quant. Elect. 1 (1995) 213.

[2] V. Dominic, S. MacCormack, R. Waarts, S. Sanders, S. Bicknese, R. Dohle, E. Wolak, P. S. Yeh, and E. Zucker, 110W fibre laser, IEEE Elect. Lett. 35 (1999) 1158-1160.

[3] Y. Jeong, J. K. Sahu, D. N. Payne, and J. Nilsson, Ytterbium-doped large-core fibre laser with $610 \mathrm{~W}$ of near diffraction-limited output power, IEEE Elect. Lett. 40 (2004) 1527-1528.

[4] Y. Jeong, J. K. Sahu, D. N. Payne, and J. Nilsson, Ytterbium-doped large-core fiber laser with $1.36 \mathrm{~kW}$ continuous-wave output power, Opt. Exp. 12 (2004) 6088-6092.

[5] Y. Jeong, J. K. Sahu, D. N. Payne, and J. Nilsson, Ytterbium-doped large-core fibre laser with $1 \mathrm{~kW}$ of continuous-wave output power, IEEE Elect. Lett. 40 (2004) 470-472.

[6] Y. Wang, Heat dissipation in kilowatt fiber power amplifiers, IEEE J. Quant. Elect. 40 (2004) 731-740.

[7] M. K. Davis, M. J. F. Digonnet, and R. H. Pantell, Thermal effects in doped fibers, J. Light. Tech. 16 (1998) 1013-1023.

[8] M. J. F. Digonnet, R. W. Sadowski, H. J. Shaw, and R. H. Pantell, Resonantly enhanced nonlinearity in doped fibers for low-power all-optical switching: A review, Opt. Fib. Tech. 3 (1997) 44-64. 
[9] S. Chenais, F. Balembois, F. Druon, G. Lucas-Leclin, and P. Georges, Thermal lensing in diode-pumped ytterbium lasers - Part I: Theoretical analysis and wavefront measurements, IEEE J. Quant. Elect. 40 (2004) 1217-1234.

[10] S. Chenais, F. Balembois, F. Druon, G. Lucas-Leclin, and P. Georges, Thermal lensing in diode-pumped ytterbium lasers - Part II: Evaluation of quantum efficiencies and thermo-optic coefficients, IEEE J. Quant. Elect. 40 (2004) 12351243.

[11] B. Wu, P. L. Chu, and J. Arkwright, Ytterbium-doped silica slab waveguide with large nonlinearity, IEEE Phot. Tech. Let. 7 (1995) 1450-1452.

[12] J. W. Arkwright, P. Elango, T. W. Whitbread, and G. R. Atkins, Nonlinear phase changes at $1310 \mathrm{~nm}$ and $1545 \mathrm{~nm}$ observed far from resonance in diode pumped ytterbium doped fiber, IEEE Phot. Tech. Let. 8 (1996) 408-410.

[13] J. W. Arkwright, P. Elango, G. R. Atkins, T. Whitbread, and M. J. F. Digonnet, Experimental and theoretical analysis of the resonant nonlinearity in ytterbiumdoped fiber, J. Light. Tech. 16 (1998) 798-806.

[14] M. J. F. Digonnet, R. W. Sadowski, H. J. Shaw, and R. H. Pantell, Experimental evidence for strong UV transition contribution in the resonant nonlinearity of doped fibers, J. Light. Tech. 15 (1997) 299-303.

[15] E. Bochove, Nonlinear refractive index of a rare-earth-doped fiber laser, Opt. Lett. 29 (2004) 2414-2416.

[16] R. H. Pantell and M. J. F. Digonnet, A Model of Nonlinear All-Optical Switching in Doped Fibers, J. Light. Tech. 12 (1994) 149-156.

[17] S. A. Boothroyd, A. Skirtach, L. Chan, and A. Akmaloni, Measurement of realtime gain gratings in erbium-doped fiber, IEEE J. Quant. Elect. 35 (1999) 39-46. 
[18] S. M. Lima, H. Jiao, L. A. O. Nunes, and T. Catunda, Nonlinear refraction spectroscopy in resonance with laser lines in solids, Opt. Lett. 27 (2002) 845847.

[19] S. M. Lima, D. N. Messias, L. A. O. Nunes, and T. Catunda, Nonlinear spectroscopy of rare earth doped materials, Anais de Óptica - XXVIII Encontro Nacional de Física da Matéria Condensada (2005) 243 - 246.

[20] R. W. Boyd, Nonlinear Optics, Academic Press, San Diego, 1992.

[21] V. S. Butylkin, A. E. Kaplan, and Y. Khronopulo, Nonlinear Polarizability in Resonant Interactions of an Electromagnetic Field with Matter, Sov. Phys. JETP $32(1971) 501-507$.

[22] R. C. Powell, S. A. Payne, L. L. Chase, and G. D. Wilke, 4-Wave Mixing of Nd3+-Doped Crystals and Glasses, Phys. Rev. B 41 (1990) 8593-8602.

[23] O. L. Antipov, D. V. Bredikhin, O. N. Eremeykin, A. P. Savikin, E. V. Ivakin, and A. V. Sukhadolau, Electronic mechanism for refractive-index changes in intensively pumped Yb : YAG laser crystals, Opt. Lett. 31 (2006) 763-765.

[24] G. Lei, J. E. Anderson, M. I. Buchwald, B. C. Edwards, R. I. Epstein, M. T. Murtagh, and G. H. Sigel, Spectroscopic evaluation of Yb3+-doped glasses for optical refrigeration, IEEE J. Quant. Elect. 34 (1998) 1839-1845.

[25] R. C. Powell, S. A. Payne, L. L. Chase, and G. D. Wilke, Index-of-Refraction Change in Optically Pumped Solid-State Laser Materials, Opt. Lett. 14 (1989) 1204-1206. 


\section{Capítulo 7}

\section{Conclusões gerais e perspectivas}

Neste trabalho nos propusemos a estudar as propriedades térmicas e eletrônicas de sólidos dopados com íons emissores. Para isto utilizamos as técnicas de Lente Térmica e z-scan resolvidas no tempo. Estas propriedades são importantes para a determinação da variação do índice de refração nestes materiais e das condições de estabilidade de dispositivos óticos baseados neles.

De uma maneira geral as principais contribuições desta tese foram: a determinação do espectro térmico e de importantes propriedades fototérmicas de amostras vítreas dopadas com $\mathrm{Yb}^{3+}$; o estudo dos efeitos da saturação da não linearidade sobre a determinação das propriedades eletrônicas em sólidos dopados com $\mathrm{Cr}^{3+}$. Resumindo, as maiores contribuições desta tese foram o aperfeiçoamento das técnicas utilizadas e sua aplicação em novos materiais. Os resultados obtidos estão de acordo com os objetivos iniciais, pois o conhecimento aqui gerado pode ser usado como base para o desenvolvimento/aperfeiçoamento de dispositivos baseados nestes íons, ou como base para pesquisas mais avançadas sobre os fenômenos estudados na tese.

Assim, dentre os trabalhos futuros que podem ser realizados baseados nesta tese podemos listar:

- Determinar os espectros térmico e eletrônico de íons de interesse tecnológico como $\mathrm{Er}^{3+}$, que é bastante usado como amplificador ótico para fibras em sistemas de telecomunicações e $\mathrm{Cr}^{3+}$ em cristais fluoretos, que são empregados na confecção de lasers sintonizáveis;

- Modificar o modelo que descreve $\mathrm{n}_{2}$ para aplicá-lo em novas situações. Com o desenvolvimento constante dos sistemas amplificadores e lasers de alta potência, 
torna-se necessário um modelo que descreva a não linearidade ótica destes sistemas sob intenso bombeamento. Este modelo deve considerar o acoplamento térmico entre os níveis de interesse e efeitos de transferência de energia como o processo de upconversion Auger;

- Estudar processos de refrigeração ótica em materiais dopados com $\mathrm{Yb}^{3+}$ usando a técnica de LT. Isto pode ser feito pois uma vez conhecidas as propriedades fototérmicas de um material dopado com $\mathrm{Yb}^{3+}$, pode-se escolher um comprimento de onda onde $\lambda>\lambda_{\text {em }}$ e usar a técnica de LT para determinar a eficiência de resfriamento, $\phi$. 


\section{Apêndice A}

\section{Características espectroscópicas de sólidos dopados com $\mathrm{Cr}^{3+}, \mathrm{Nd}^{3+}$ e $\mathrm{Yb}^{3+}$}

Neste trabalho foram utilizadas amostras dopadas com os íons emissores $\mathrm{Cr}^{3+}, \mathrm{Nd}^{3+}$ e $\mathrm{Yb}^{3+}$, de modo que abordaremos aqui as características espectroscópicas destes materiais, pois elas serão citadas ao longo dos capítulos da tese.

\section{A.1. Materiais dopados com $\mathrm{Cr}^{3+}$}

O íon $\mathrm{Cr}^{3+}$ tem a mesma estrutura eletrônica do Ar mais três elétrons na camada de valência externa 3d. Os seus níveis de energia são bem conhecidos tanto no caso do íon livre como para o íon no campo cristalino.

O rubi $\left(\mathrm{Al}_{2} \mathrm{O}_{3}: \mathrm{Cr}^{3+}\right)$ é a safira dopada com $\mathrm{Cr}^{3+}$, onde os íons de $\mathrm{Cr}^{3+}$ substituem uma pequena parte dos íons de $\mathrm{Al}^{3+}$. A rede hospedeira possui um célula unitária hexagonal cujo eixo de simetria define o eixo ótico do cristal. Tipicamente a concentração de $\mathrm{Cr}$ é $0.05 \%$ em peso o que equivale a $\sim 1.9 \times 10^{19} \mathrm{Cr}^{3+} / \mathrm{cm}^{3}$. O espectro de absorção do rubi apresenta duas bandas largas com largura 100 nm, correspondentes aos estados ${ }^{4} \mathrm{~T}_{2}$ e ${ }^{4} \mathrm{~T}_{1}$ em $18000 \mathrm{~cm}^{-1}$ (555nm) e $25000 \mathrm{~cm}^{-1}(400 \mathrm{~nm})$, Figura A-1. O rubi apresenta ainda uma forte absorção no ultra violeta correspondente a banda de transferência de carga. 


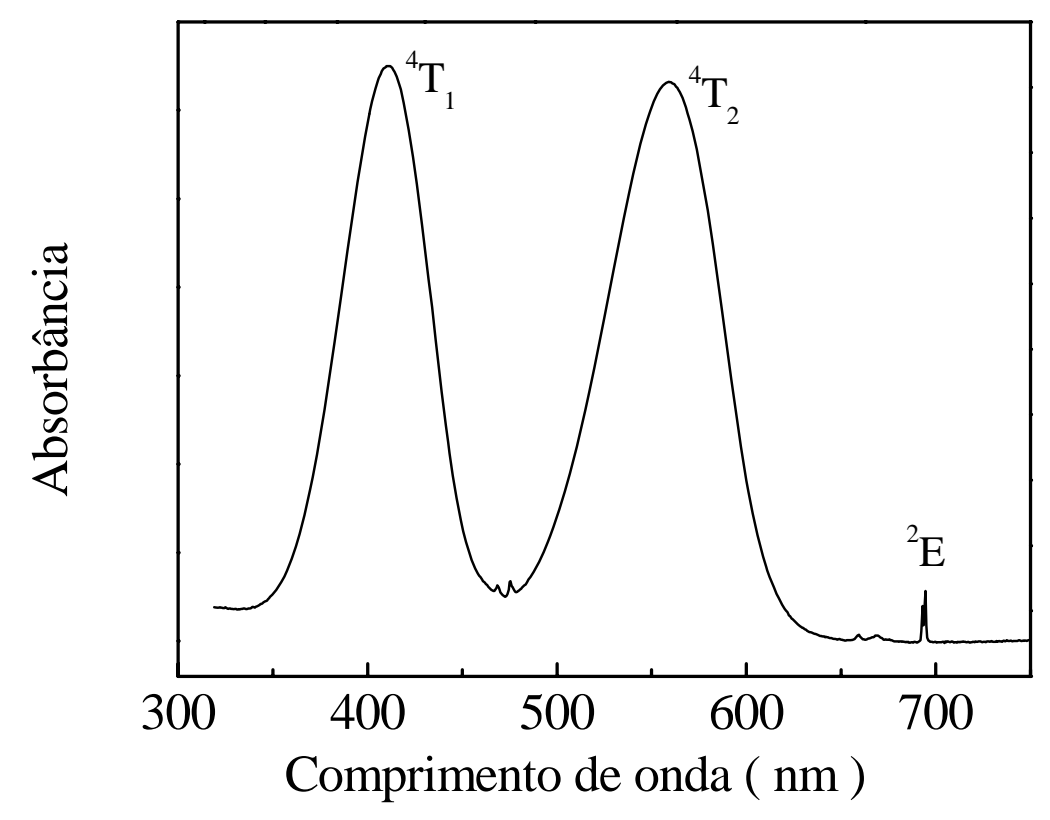

Figura A-1 - Espectro de absorção do cristal de rubi com E $\perp$ c.

Para a emissão laser, normalmente excitam-se as bandas ${ }^{4} \mathrm{~T}_{2}$ e ${ }^{4} \mathrm{~T}_{1}$ através de uma lâmpada de flash. A partir destas bandas os íons decaem não-radiativamente para o dubleto ${ }^{2} \mathrm{E}$ com uma taxa de relaxação $\mathrm{W} \sim 5 \times 10^{-8} \mathrm{~s}^{-1}$. O dubleto ${ }^{2} \mathrm{E}$ é constituído por dois níveis 2A e E separados por $29 \mathrm{~cm}^{-1}$ devido a distorção trigonal do campo octaédrico, Figura A-2. A partir dos níveis ${ }^{2} \mathrm{E}$ a transição para o estado fundamental ${ }^{2} \mathrm{E} \rightarrow{ }^{4} \mathrm{~A}_{2}$ (as linhas R) é proibida por spin, por isso apresenta um tempo de vida radiativo bastante longo $\tau_{\mathrm{o}} \sim 3,8 \mathrm{~ms}$. As linhas R são linhas de zero- fonon em 694,3 nm e 692,5 nm por isso são relativamente estreitas $\left(\Delta v \sim 6 \mathrm{~cm}^{-1}\right.$ a $\left.300 \mathrm{~K}\right)$. 


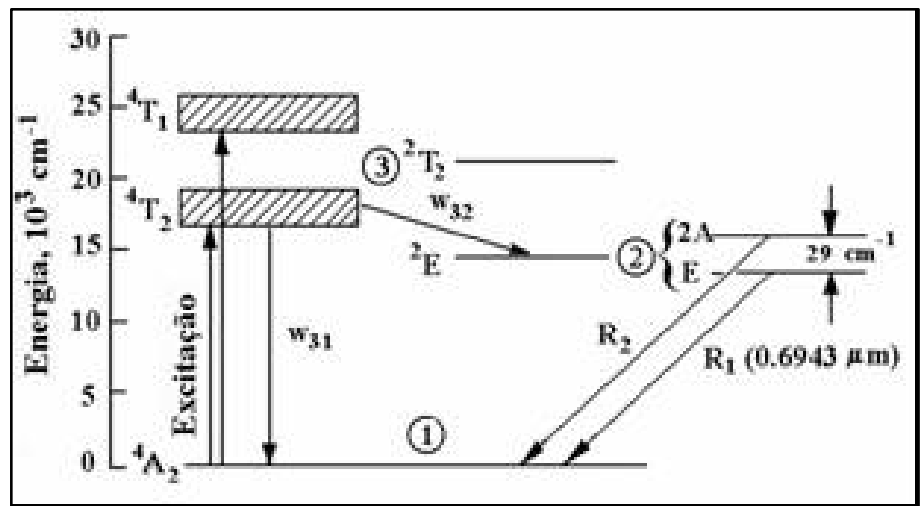

Figura A-2 - Diagrama de níveis do rubi, mostrando as possíveis energias de excitação e as linhas $\mathrm{R}$ laser.

Atualmente existe também um grande interesse em cristais fluoretos dopados com $\mathrm{Cr}^{+3}$ devido ao fato de terem bandas de absorção no vermelho, que podem ser excitadas por lasers de diodo em 670nm, e apresentarem um largo intervalo de sintonia em torno de 800nm. Seu largo espectro de emissão torna-os também atrativos para produção de pulsos de fentosegundos.

O cristal LiSAF $\left(\mathrm{LiSrAlF}_{6}: \mathrm{Cr}^{+3}\right)$ pertence à da família dos fluoretos e é comumente denominados de colquiriite. ${ }^{1}$ O LiSAF apresenta um tempo de vida de fluorescência de $67 \mu$ s $\left(\mathrm{T}=23^{0} \mathrm{C}\right)$, constante desde $20 \mathrm{~K}$ até a temperatura ambiente. Na Figura A-3 são mostrados os espectros de absorção (polarizado) e emissão para o cristal de LiSAF, realizados à temperatura ambiente.

\footnotetext{
${ }^{1} \mathrm{O}$ nome advém de Colquiri, cidade da Bolívia em que esses materiais foram descobertos.
} 


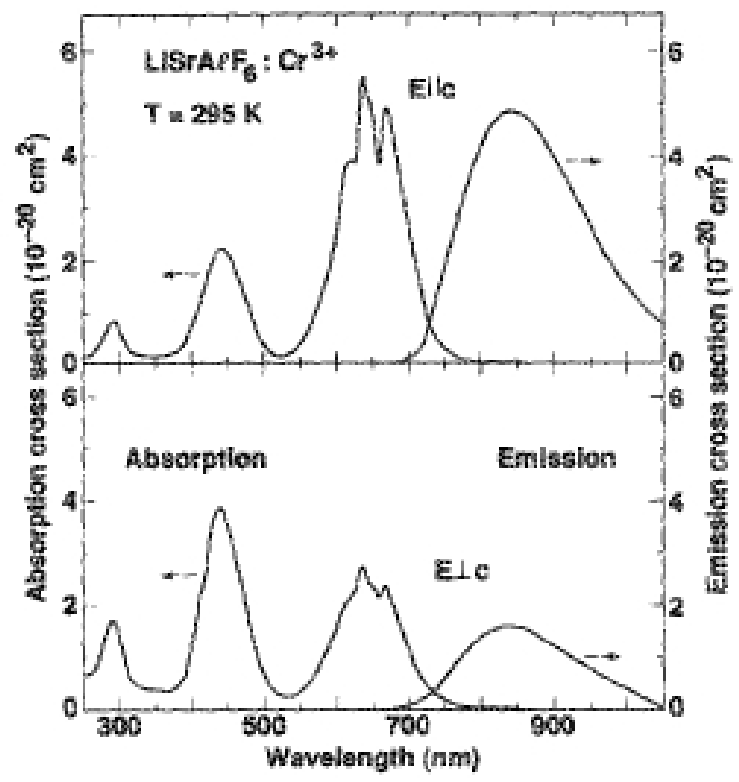

Figura A-3- Espectros das seções de choque de absorção e emissão dos cristal de $\mathrm{Cr}^{3+}$ :LiSAF .

Note que ambos os espectros apresentam uma banda larga centrada em aproximadamente $440 \mathrm{~nm}$, devido a transição ${ }^{4} \mathrm{~A}_{2} \rightarrow{ }^{4} \mathrm{~T}_{1}$, e outra banda por volta de 650 $\mathrm{nm}$, relacionada a transição ${ }^{4} \mathrm{~A}_{2} \rightarrow{ }^{4} \mathrm{~T}_{2}$. A banda em aproximadamente $440 \mathrm{~nm}$ é mais intensa para a polarização do campo elétrico $E \perp c$, enquanto a banda na região de 650 nm é mais intensa para Ellc. É interessante notar que os comprimentos de onda dos picos das bandas ${ }^{4} \mathrm{~T}_{1}(440 \mathrm{~nm}) \mathrm{e}^{4} \mathrm{~T}_{2}(650 \mathrm{~nm})$, nesses fluoretos são bem maiores do que no rubi, que são 400 e 550 nm, respectivamente.

\section{A.2. $\quad$ Materiais dopados com $\mathrm{Nd}^{3+}$}

Neodímio trivalente, $\mathrm{Nd}^{3+}$, é atualmente o mais bem sucedido tipo de íon ativo para lasers de estado sólido. Também é o íon onde mais se obteve ação laser para diferentes materiais. Ele pode operar tanto no regime pulsado como no de onda 
contínua, com uma linha estreita de emissão. A emissão mais comum é próximo a $1 \mu \mathrm{m}$ $\left({ }^{4} \mathrm{~F}_{3 / 2} \rightarrow{ }^{4} \mathrm{I}_{11 / 2}\right)$, mas existem várias outras possibilidades de emissão laser no infravermelho (1,8 $\mu \mathrm{m} \quad{ }^{4} \mathrm{~F}_{3 / 2} \rightarrow{ }^{4} \mathrm{I}_{15 / 2} ; 1,35 \mu \mathrm{m}{ }^{4} \mathrm{~F}_{3 / 2} \rightarrow{ }^{4} \mathrm{I}_{13 / 2}$ e $\left.0,88 \mu \mathrm{m}{ }^{4} \mathrm{~F}_{3 / 2} \rightarrow{ }^{4} \mathrm{I}_{9 / 2}\right)$. Embora os efeitos de diferentes hospedeiros sejam mais sutis sobre o $\mathrm{Nd}^{3+}$ do que sobre os metais de transição, eles ainda podem levar a diferentes características laser, e. $g$. comprimento de onda, devido a mudanças em propriedades importantes como força da transição radiativa, absorção de estado excitado, processos de relaxação cruzada, etc. Em relação ao rubi, eles apresentam a vantagem de funcionarem como laser de 4 níveis e por isso apresentam baixa condição de limiar (“threshold”). Além disso, apresentam alta seção de choque na transição laser, por exemplo no $\mathrm{Nd}^{+3}$ :YAG $\left(\mathrm{YAG}=\mathrm{Y}_{3} \mathrm{Al}_{5} \mathrm{O}_{12}\right)$ $\sigma=9 \times 10^{-19} \mathrm{~cm}^{2}, 7$ vezes maior que a do rubi. A Figura A-4 mostra o espectro de absorção do íon de $\mathrm{Nd}^{3+}$ no cristal de YAG, onde chama a atenção as linhas estreitas de absorção.

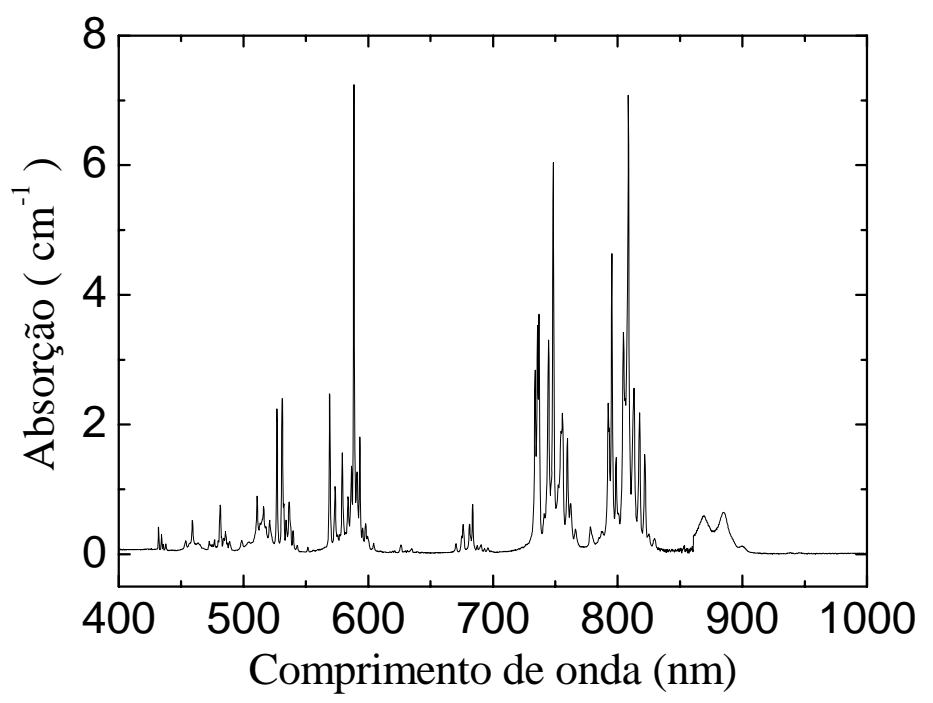

Figura A-4 - Espectro de absorção de uma amostra de $\mathrm{Nd}^{3+}$ :YAG com uma concentração de $1,03 \times 10^{20} \mathrm{~cm}^{-3}$.

A Figura A-5 mostra o diagrama de níveis do $\mathrm{Nd}^{3+}$ no cristal de YAG. Neste diagrama estão mostrados os principais comprimentos de onda de excitação, assim 
como as emissões do íon. No entanto, com o advento dos lasers de diodo de alta potência, também o bombeamento em $869 \mathrm{~nm}$, ressonante ao nível laser ${ }^{4} \mathrm{~F}_{3 / 2}$, se tornou uma alternativa viável apesar de sua baixa seção de choque de absorção (comparada à seção em $808 \mathrm{~nm}$ ). Dentre as emissões do $\mathrm{Nd}^{3+}$ :YAG, a mais utilizada é a ${ }^{4} \mathrm{~F}_{3 / 2} \rightarrow{ }^{4} \mathrm{I}_{13 / 2}$ em $1064 \mathrm{~nm}$.

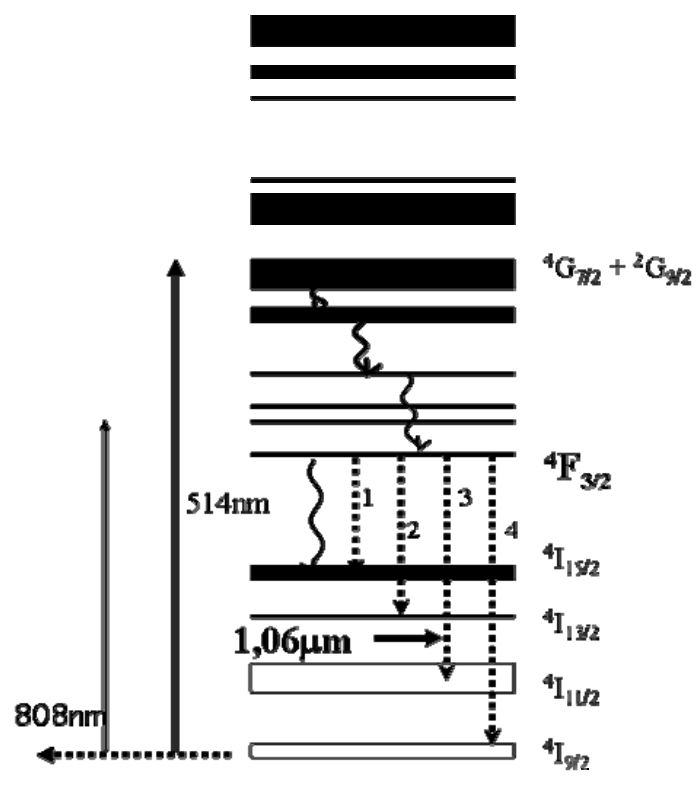

Figura A-5 - Diagrama de níveis para o $\mathrm{Nd}^{3+}$ :YAG mostrando os principais comprimentos de onda de excitação e as linhas laser obtidas. As setas onduladas indicam possíveis decaimentos não radiativos.

Quando colocado numa estrutura vítrea o íon está submetido à variação do campo cristalino devido à estrutura amorfa do vidro. Por isso existe um grande alargamento não homogêneo das linhas, ou seja, íons que “sentem” campos diferentes emitem em comprimentos de onda diferentes. No $\mathrm{Nd}^{+3}$ :YAG, por exemplo, a largura de linha da transição laser é $6 \mathrm{~cm}^{-1}$ enquanto que num vidro este valor é tipicamente 50 vezes maior. 


\section{A.3. Materiais dopados com $\mathrm{Yb}^{3+}$}

Apesar de todas as suas vantagens os íons de $\mathrm{Nd}^{3+}$ ainda estão sujeitos ao problema da geração de calor, devida a processos não radiativos como transferência ascendente de energia (também conhecida como upconversion Auger), relaxação cruzada, defeito quântico, etc. Uma maneira de diminuir consideravelmente o impacto do aquecimento devido ao bombeio é escolher um meio de ganho que gere menos calor. Graças ao desenvolvimento dos lasers de diodo emitindo em torno de $970 \mathrm{~nm}$, os materiais dopados com itérbio tem se tornado importantes a ponto de competir com aqueles dopados com $\mathrm{Nd}^{3+}$. Os íons de itérbio trivalente, $\mathrm{Yb}^{3+}$, possuem apenas dois níveis acessíveis oticamente: o estado fundamental ${ }^{2} \mathrm{~F}_{7 / 2}$ e o estado excitado ${ }^{2} \mathrm{~F}_{5 / 2}$. Estes níveis têm uma separação de $10000 \mathrm{~cm}^{-1}$ o que torna o decaimento não radiativo extremamente improvável, levando a eficiência quântica de fluorescência, ๆ, a quase 100\%. Além disso, devido ao seu tempo de vida relativamente longo, 2 ms, e sua larga banda de emissão é possível usar este sistema como laser de baixa taxa de repetição, pulso ultra-curto, e/ou sintonizável no infravermelho. Outra importante aplicação destes íons é a refrigeração ótica. Neste caso a condição básica de funcionamento é que o material tenha uma alta eficiência quântica de fluorescência anti-Stokes, i. e., o comprimento de onda da excitação deve ser maior que o comprimento de onda médio do fóton fluorescente. Esta condição é pode ser satisfeita pelo íon de $\mathrm{Yb}^{3+}$. A Figura A7 mostra o espectro de absorção e o diagrama de níveis do $\mathrm{Yb}^{3+}$ no vidro fosfato $\mathrm{QX} / \mathrm{Yb}$. 


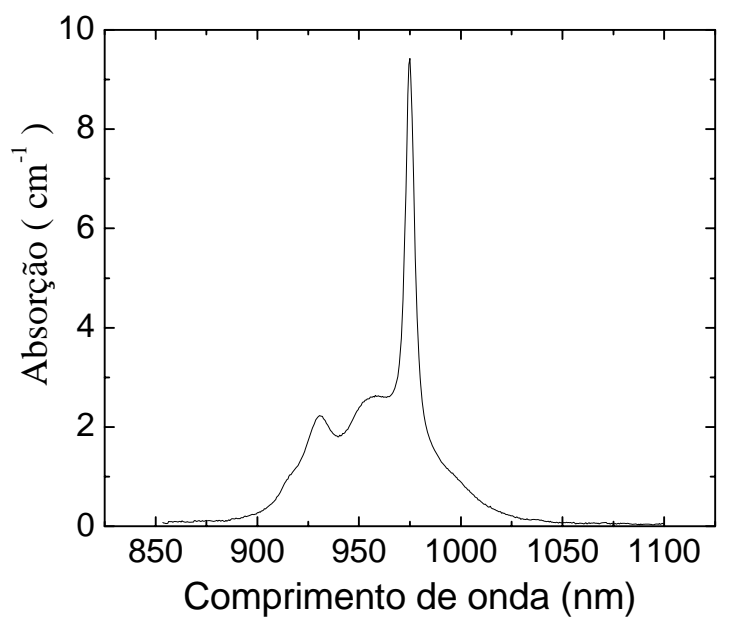

(a)

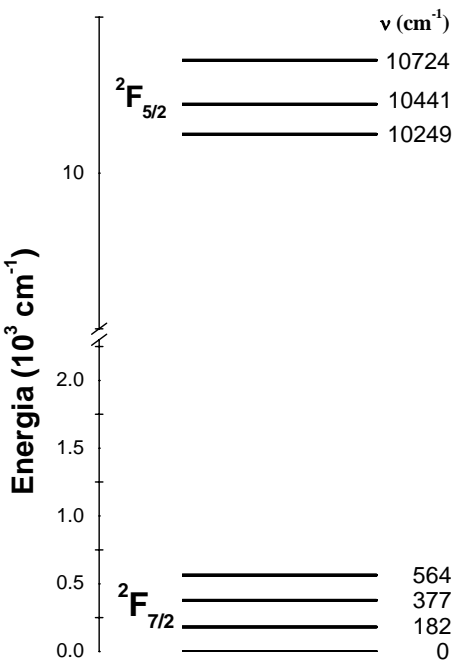

(b)

Figura A-6 - (a) Espectro de absorção do íon de $\mathrm{Yb}^{3+}$ no vidro fosfato QX/Yb. (b) Diagrama de níveis de energia do $\mathrm{Yb}^{3+}$ no mesmo vidro mostrando a posição de cada subnível.

Da Figura A-7(a) vemos que o $\mathrm{Yb}^{3+}$ possui uma banda de absorção de aproximadamente $100 \mathrm{~nm}$. As estruturas dentro da banda devem-se à separação entre os subníveis de energia dos estados excitado e fundamental. A divisão dos níveis eletrônicos sob a ação do campo cristalino, Figura A-7(b), permite ao $\mathrm{Yb}^{3+}$ ser operado no esquema de quase três níveis. Como conseqüência, o defeito quântico é pequeno, tipicamente menor que $10 \%$. Assim, espera-se que a maior contribuição para a variação do índice de refração venha dos efeitos eletrônicos. 


\section{Apêndice B}

\section{Integral de difração de Kirchhoff}

Inicialmente, consideremos um campo monocromático na aproximação escalar em que os campos estão uniformemente (linear ou circularmente) polarizados. Seja o campo elétrico dado por:

$$
E(\vec{r}, t)=\varepsilon(\vec{r}) e^{-i \omega t}
$$

sendo que em coordenadas cartesianas $\vec{r} \vec{r}=(x, y, z)$. A amplitude do campo $\varepsilon$ que satisfaz a equação de onda, é expressa por:

$$
\left(\nabla^{2}+k^{2}\right) \varepsilon(\vec{r})=0
$$

sendo $\mathrm{k}=\omega / \mathrm{c}=2 \pi / \lambda$, pois o campo propaga no vácuo ou no $\operatorname{ar}\left(\mathrm{n}_{\mathrm{o}} \approx 1\right)$.

Consideremos agora, que este campo esteja propagando-se na direção dada pelo versor $\hat{n}$ e seja difratado por um orifício finito num plano opaco infinito $S$ (Figura B.1). Supomos que o orifício no plano $\mathbf{S}$ seja descrito pelas coordenadas $\left(x_{1}, y_{1}, z\right)$, e $\left(x_{2}, y_{2}\right.$, $z+d)$, as coordenadas no plano de observação $\boldsymbol{P}$, situado a uma distância $d_{01}$ do orifício. A amplitude complexa do campo $\varepsilon\left(r_{2}\right)$ num ponto $\mathrm{P}_{2}$ do plano de observação pode ser obtida pela somatória do campo $\varepsilon_{\mathrm{s}}$ devido a todos os pontos $\mathrm{P}_{1}$ do plano do orifício. Esta é a idéia básica do princípio de Huygens-Fresnel, segundo o qual cada ponto da frente de onda é o centro de uma perturbação que origina uma onda esférica, e estas novas ondas esféricas interferem para definir a frente de onda num instante posterior. Matematicamente, esta idéia é expressa pela integral de Fresnel-Kirchhoff, na forma: 


$$
\varepsilon\left(\mathrm{x}_{2}, \mathrm{y}_{2}\right)=\frac{\mathrm{i}}{\lambda} \iint_{\mathrm{s}} \frac{\mathrm{e}^{-\mathrm{ikd}} 01}{\mathrm{~d}_{01}} \cos \left(\mathbf{n} \cdot \mathbf{d}_{\mathbf{0 1}}\right) \varepsilon_{\mathrm{s}}\left(\mathrm{x}_{1}, \mathrm{y}_{1}\right) \mathrm{dx} \mathrm{d}_{1} \mathrm{dy}{ }_{1}
$$

em que $\lambda$ é o comprimento de onda da luz. Para a integral acima ser uma ferramenta efetiva, faz-se algumas aproximações válidas para pequenos ângulos, como considerar a distância $d$ muito maior que o máximo da dimensão linear da abertura no plano $S, \mathrm{r}_{1}$ $<<\mathrm{d}\left(\mathrm{r}_{1}^{2}=\mathrm{x}_{1}^{2}+\mathrm{y}_{1}{ }^{2}\right)$, e posicionar a fonte de luz centralmente localizada com relação a abertura da fenda $\left(\cos \left(\mathbf{n}_{\mathbf{d}} \mathbf{d}_{\mathbf{0 1}}\right)=1\right)$.

Nosso interesse é estudar problemas com simetria cilíndrica onde a amplitude do campo incidente é expressa por $\varepsilon_{\mathrm{s}}\left(\mathrm{x}_{1}, \mathrm{y}_{1}\right)=\varepsilon_{\mathrm{s}}\left(\mathrm{r}_{1}\right)$ no plano $\mathrm{S}$, e o campo no plano $\boldsymbol{P}$ de observação $\varepsilon\left(\mathrm{x}_{2}, \mathrm{y}_{2}\right)=\varepsilon\left(\mathrm{r}_{2}\right)$, com $\mathrm{r}_{2}{ }^{2}=\mathrm{x}_{2}{ }^{2}+\mathrm{y}_{2}{ }^{2}$. A (B-3 pode ser resolvida utilizando coordenadas cilíndricas devido a simetria radial ou cilíndrica do feixe de luz analisado. Desta forma, na aproximação de Fresnel obtemos:

$$
\varepsilon\left(r_{2}\right)=\frac{i k}{d} e^{-i k\left(d+\frac{r_{2}^{2}}{2 d}\right)} \int_{0}^{\infty} e^{-\frac{i k r}{2 d}} \varepsilon_{s}\left(r_{1}\right) J_{0}\left(\frac{k r_{1} r_{2}}{d}\right) r_{1} d r_{1}
$$

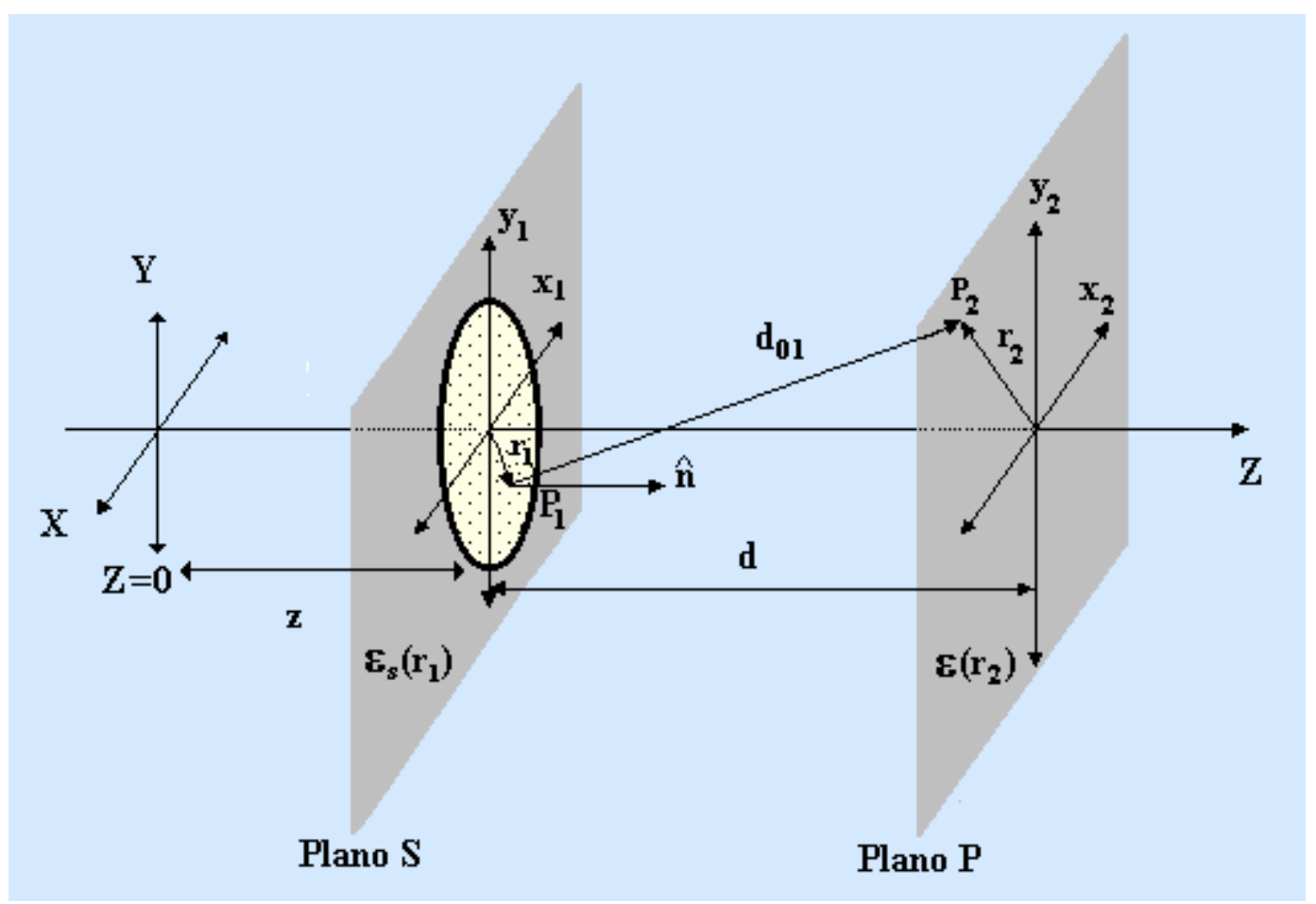

Figura B.1 - Geometria da Difração de Fresnel-Kirchhoff. 
Consideremos agora o caso em que o campo incidente é um feixe Gaussiano de ordem zero $\left(\mathrm{TEM}_{00}\right)$. A propagação de um feixe Gaussiano pode ser abordada pela integral de difração, substituindo a amplitude $\varepsilon_{\mathrm{s}}\left(\mathrm{r}_{1}, \mathrm{z}\right)$ para um feixe Gaussiano no modo $\mathrm{TEM}_{00}$, expresso por:

$$
\varepsilon_{S}\left(r_{1}, z\right)=A_{0} \frac{w_{0}}{w_{1}(z)} \exp -\left[\frac{r_{1}^{2}}{w_{1}^{2}(z)}+i \frac{k r_{1}^{2}}{2 R_{1}(z)}+i \tan ^{-1}\left(z / z_{0}\right)\right]
$$

em que $w_{0}$ é o raio na cintura do feixe $(Z=0), R_{1}$ é o raio de curvatura do feixe no plano S, o chamado comprimento de Rayleigh é definida por $\mathrm{z}_{\mathrm{c}}=\mathrm{n}_{0} \pi \mathrm{w}_{0}{ }^{2} / \lambda$, em que $\mathrm{n}_{0}$ é o índice de refração do meio ( $\mathrm{n}_{0} \approx 1$, para o ar). É conveniente escrevermos a distância $\mathrm{z}$ (fora da posição $\mathrm{Z}=0$ ao plano $\mathbf{S}$ ) em termos de uma nova variável adimensional $\mathrm{x} \equiv \mathrm{Z} / \mathrm{Z}_{0}$, $\operatorname{logo} \mathrm{w}_{1}(\mathrm{x})$ e $\mathrm{R}_{1}(\mathrm{x})$ no plano $\mathrm{S}$ são dadas por:

$$
w_{1}^{2}(x)=w_{0}^{2}\left(1+x^{2}\right)
$$

e

$$
R_{1}(x)=\left(z_{c} / x\right)\left(1+x^{2}\right)
$$

É importante notar que em $\mathrm{r}_{1}=\mathrm{w}_{1}$ a amplitude do campo cai por um fator de $1 / \mathrm{e}$, ou seja, a intensidade cai por $1 / \mathrm{e}^{2}$. Denomina-se $\mathrm{w}_{1}$ o valor do raio do feixe, ou $2 \mathrm{w}_{1} \mathrm{o}$ valor de sua cintura (“beam waist”) no plano S, sendo mínimo na posição Z=0.

Na Figura B.2 é representada a propagação de um feixe Gaussiano. A propagação desse tipo de feixe não segue as leis de óptica geométrica devido à difração da luz no ponto focal, sendo dessa forma muito utilizado em investigações de óptica não linear. A difração causa um alargamento transversal do feixe propagado e o ângulo $\theta^{*}$ de difração é dado por $\theta^{*}=\mathrm{w}_{1}(\mathrm{z}) / \mathrm{z}=\lambda / \pi \mathrm{w}_{0}$. No caso $\mathrm{z}$ muito grande $\mathrm{w}_{1}(\mathrm{z}) \approx \lambda \mathrm{z} / \pi \mathrm{w}_{0}$ é 
aproximadamente constante. O parâmetro R (Eq. (2.14)) é o raio de curvatura da frente de onda esférica em $\mathrm{z}$ (note que $\mathrm{R}<0$ em $\mathrm{z}<0$ e $\mathrm{R}>0$ para $\mathrm{z}>0$ ).

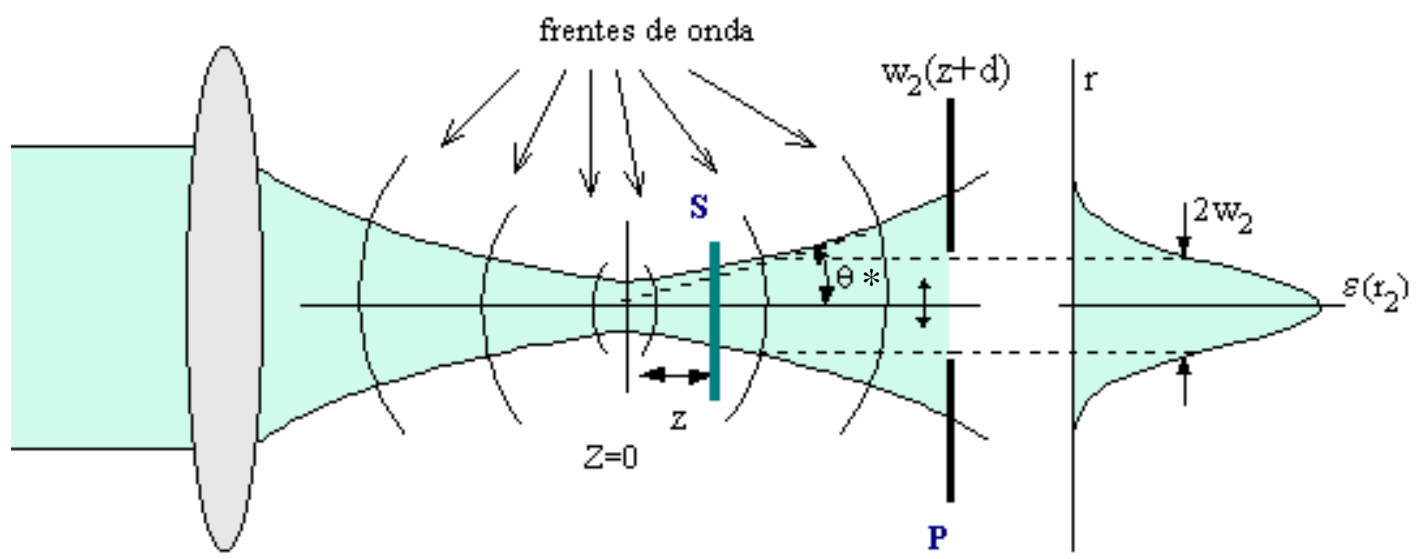

Figura B.2 - Propagação de um feixe Gaussiano, em que $\mathbf{S}$ representa o plano em que situa a amostra, a uma distância d de $\mathbf{P}$ (plano de observação). A figura mostra à direita o perfil de intensidade $\varepsilon\left(\mathrm{r}_{2}\right)$ do feixe de laser no modo $\mathrm{TEM}_{00}$, com ângulo de difração $\theta$.

Mostraremos agora que podemos calcular a amplitude de um feixe Gaussiano no campo distante. A integral de difração por substituição de $\rho_{1} \equiv \mathrm{r}_{1} / \mathrm{w}_{1}$ e $\psi \equiv \mathrm{kw}_{1} \mathrm{r}_{2} / \mathrm{d}$, pode ser escrita na forma:

$$
\varepsilon\left(r_{2}\right)=A^{\prime} e^{-\frac{i k r_{2}^{2} 2 d}{\infty}} \int_{0}^{\infty} e^{-\rho_{1}^{2}(1+i C)} j_{0}\left(\psi \rho_{1}\right) \rho_{1} d \rho_{1}
$$

em que

$$
\begin{aligned}
& A^{\prime}=\frac{i k}{d} A_{0} w_{0} w_{1}(x) e^{-i \tan ^{-1}(x)} e^{-i k d} \\
& C=\frac{k w_{1}^{2}}{2}\left(\frac{1}{d}+\frac{1}{R_{1}}\right)
\end{aligned}
$$

Utilizando a integral resolvida abaixo, na forma: 


$$
\int_{0}^{\infty} \mathrm{e}^{-\rho_{1}^{2}(1+\mathrm{iC})} \mathrm{J}_{0}\left(\psi \rho_{1}\right) \rho_{1} \mathrm{~d} \rho_{1}=\frac{\mathrm{e}^{\left(-\frac{\psi^{2}}{4(1+\mathrm{iC})}\right)}}{2(1+\mathrm{iC})}
$$

e substituindo $\mathrm{k}=2 \mathrm{z}_{\mathrm{c}} / \mathrm{w}_{0}{ }^{2}$ (sendo $\mathrm{z}_{\mathrm{c}}=\pi \mathrm{w}_{0}{ }^{2} \mathrm{n}_{0} / \lambda$ e $\lambda=2 \pi_{0} / \mathrm{k}$ ) e $\mathrm{x}_{2} \equiv(\mathrm{z}+\mathrm{d}) / \mathrm{z}_{\mathrm{c}} \approx \mathrm{d} / \mathrm{z}_{\mathrm{c}}$, pois no campo distante $\mathrm{d}>>\mathrm{z}$, obtemos:

$$
\varepsilon\left(r_{2}\right)=\frac{A^{\prime}}{2(1+i C)} e^{-\left(\frac{\psi^{2}}{4(1+i C)}+\frac{i k r_{2}^{2}}{2 d}\right)}
$$

que pode ser escrita como:

$$
\varepsilon\left(\mathrm{r}_{2}\right)=\mathrm{A}_{2} \mathrm{e}^{-\mathrm{r}_{2}^{2} / \mathrm{w}_{2}^{2}} \mathrm{e}^{-\mathrm{ikr} \mathrm{r}_{2}^{2} / 2 \mathrm{R}_{2}}
$$

com

$$
\begin{aligned}
A_{2}=\frac{A^{\prime}}{2(1+i C)} & \\
R_{2}=\frac{2 d^{2}\left(1+C^{2}\right)}{2 d\left(1+C^{2}\right)-k w_{1}^{2} C} & \mathrm{w}_{2} \approx \mathrm{w}_{0} \mathrm{x}
\end{aligned}
$$

Neste caso $A_{2}$, $w_{2}$ e $R_{2}$ representam a amplitude do campo, o raio e o raio de curvatura, respectivamente, no plano $\mathbf{P}$ de observação. 


\section{Apêndice C}

\section{Medidas das cinturas dos feixes de prova e excitação}

Para se determinar os parâmetros $m$ e $V$ utilizados na técnica de LT foram realizadas medidas das cinturas dos feixes de excitação e de prova. Para isto utilizamos um medidor de diâmetro de feixe Omega Meter - Beam Profiler,Thorlabs, modelo WM100. Partindo da teoria de propagação de feixes gaussianos, sabemos que a intensidade do feixe é dada por:

$$
\mathrm{I}(\mathrm{r})=\mathrm{I}_{0} \mathrm{e}^{-2 \mathrm{r}^{2} / \mathrm{w}^{2}(z)}
$$

onde

$$
\mathrm{w}^{2}(\mathrm{z})=\mathrm{w}_{0}^{2}\left(1+\mathrm{z}^{2} / \mathrm{z}_{\mathrm{c}}^{2}\right)
$$

sendo $\mathrm{z}_{\mathrm{c}}=\pi \mathrm{w}_{0}^{2} / \lambda$ a chamado distância ou parâmetro confocal do feixe, $w_{0}$ a cintura do feixe no ponto de máxima intensidade axial. Para determinar o valor de $w_{0}$, foi medido o diâmetro do feixe em várias posições à frente da lente passando pelo seu foco e indo um pouco além deste, tomando o cuidado de obter mais pontos na região do foco nominal da lente $(20 \mathrm{~cm})$. Fazendo o gráfico do quadrado dos valores de $w$ obtidos com $o$ medidor em função da distância da posição do medidor até a lente, temos uma parábola que pode ser ajustada pela equação $\mathrm{y}=\mathrm{a}+\mathrm{bz}+\mathrm{cz}^{2}$, fornecendo as constantes $a, b$ e $c$. O centro da parábola, $\mathrm{z}_{0}=-\mathrm{b} / 2 \mathrm{c}$, indica a posição de menor diâmetro do feixe. Na Figura C-1 temos o gráfico dos pontos obtidos experimentalmente para a determinação do diâmetro do feixe do laser de He-Ne, em 632 nm, utilizado como feixe de prova. A partir do ajuste dos dados obtivemos: $a=(0,3 \pm 0,9) \times 10^{-3}, \quad b=-(25,6 \pm 0,1) \times 10^{-3}$ e 
$C=(5,97 \pm 0,02) \times 10^{-4}$. Utilizando estes dados encontramos a posição da cintura do feixe do laser de He-Ne igual a $z_{0}=z_{0 p}=(21,4 \pm 0,2) \mathrm{cm}$.

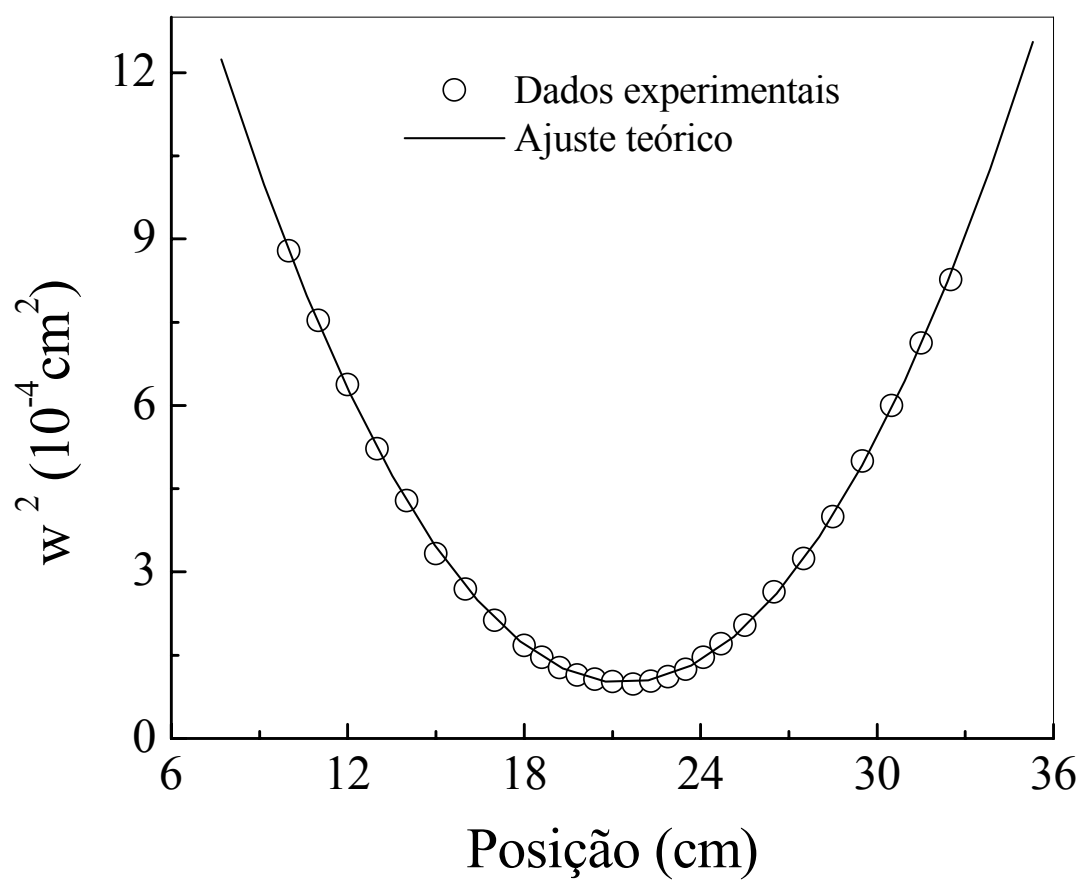

Figura C-1 - Quadrado do diâmetro do feixe do laser de HeNe em função da distância da lente convergente de foco $f=20$ $\mathrm{cm}$. Os dados experimentais foram ajustados com a equação da parábola.

De posse do valor de $\mathrm{z}_{0 \mathrm{p}}$, podemos então, a partir do mesmo gráfico determinar o valor do parâmetro confocal, $\mathrm{z}_{\mathrm{cp}}$. Para isto deslocamos a parábola $w^{2}$ para a origem $\left(z^{\prime}=\mathrm{z}-\mathrm{z}_{0 \mathrm{p}}\right)$ e ajustamos novamente a curva. Os novos valores encontrados para as constantes foram: $a=(0,01 \pm 0,1) \times 10^{-3}, b=(8,2 \pm 0,1) \times 10^{-8}$ e $c=(5,97 \pm 0,02) \times 10^{-7}$. Logo, fazendo $\mathrm{z}_{\mathrm{cp}}=\sqrt{\mathrm{a} / \mathrm{c}},{ }^{1}$ determinamos o parâmetro confocal do feixe de prova. Para o feixe de prova $z_{c p}=(4,1 \pm 0,2) \mathrm{cm}$. Finalmente, usando este valor determinamos a cintura do feixe na posição de foco, $w_{0 \mathrm{p}}=(9,1 \pm 0,2) \times 10^{-3} \mathrm{~cm}$. Seguindo o mesmo procedimento podemos determinar também a posição e a cintura do feixe de excitação ${ }^{1}$ De $w^{2}=w_{o p}^{2}+\frac{w_{o p}^{2}}{z_{c p}^{2}} z^{2}$ 
$w_{0 \mathrm{e}}=(3,5 \pm 0,1) \times 10^{-3} \mathrm{~cm}$. De posse do valor das cinturas dos feixes podemos determinar $\mathrm{o}$ valor de $m$, que para o nosso caso foi igual a 27 , usando $\lambda_{\text {exc }}=514 \mathrm{~nm}$. Em nossas medidas trabalhamos com $V=1,73$ onde se tem a otimização do sinal de LT. Por fim, para que fosse feita uma calibração do experimento, foi aferido o valor da difusividade térmica de um material já conhecido (vidro silicato dopado com $\mathrm{Fe}^{+}$).

No entanto em alguns casos onde não seja possível usar um medidor de cintura de feixes, pode-se usar uma técnica alternativa para a determinação da cintura do feixe. Por exemplo, usando uma amostra referência com $D$ já conhecido, e. g. vidro silicato dopado com $\mathrm{Fe}^{+}$, mede-se o sinal de LT para várias posições ao longo do feixe e toma-se a razão $I(t) / I(0)$ com $t>>t_{c}$ (da Equação 2-5).

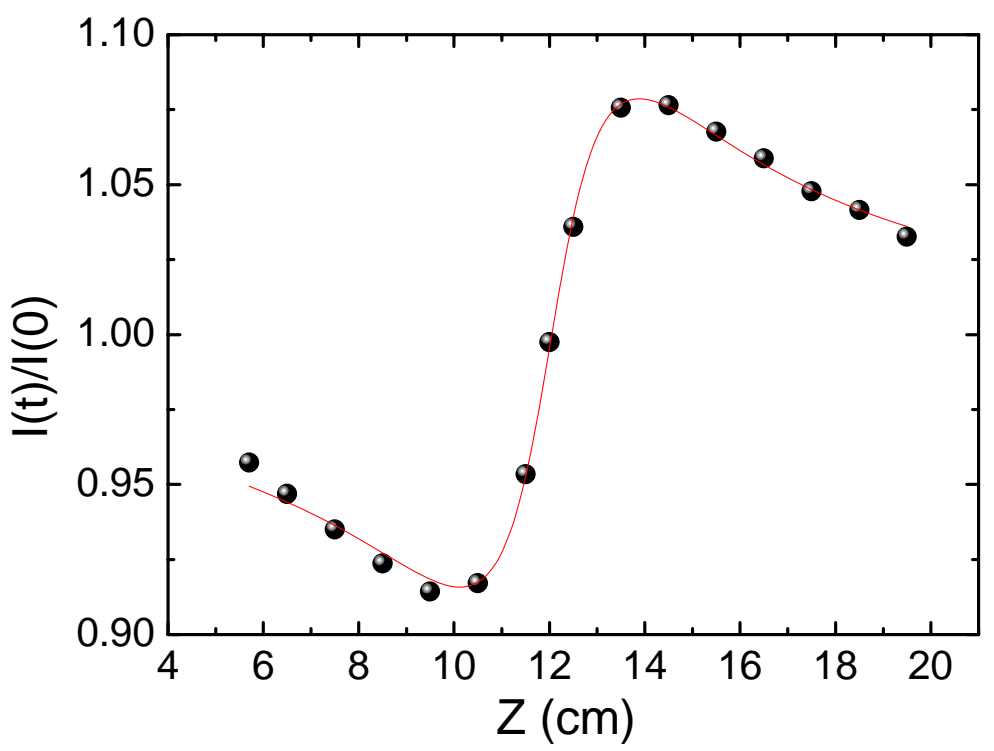

Figura C-2 - Sinal de LT como função da posição para um vidro silicato dopado com $\mathrm{Fe}^{+}$. Do ajuste com a Equação 2-5 obteve-se $\theta=(-0.156 \pm 0.002) \mathrm{rad}, \mathrm{z}_{0}=$

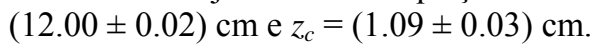

Com $t_{c}$ fixo esta equação torna-se apenas função de $z$ e $\theta$, de modo que pode ser usada para ajustar os dados experimentais e obter de uma só vez $\theta, z_{c}$ e $z_{0}$, como está ilustrado na Figura C-2. Esta técnica, assim como a anterior, pode ser aplicada tanto para o feixe de prova como para o de excitação. 